\title{
The Influence of Small-Scale \\ Anisotropies AND THE LARGE-SCALE \\ ENVIRONMENT ON THE OBSERVED \\ Properties of Lyman-Alpha Emitters
}

\author{
Dissertation \\ zur Erlangung des mathematisch-naturwissenschaftlichen \\ Doktorgrades \\ "Doctor rerum naturalium" \\ der Georg-August-Universität Göttingen \\ im Promotionsprogramm PROPHYS \\ der Georg-August University School of Science (GAUSS) \\ vorgelegt von \\ Christoph Behrens \\ aus Helmstedt \\ Göttingen, 2014
}


Betreuungsausschuss:

Jens Niemeyer, Institut für Astrophysik, Universität Göttingen

Wolfram Kollatschny, Institut für Astrophysik, Universität Göttingen

Dominik Schleicher, Institut für Astrophysik, Universität Göttingen

Prüfungskommission:

Referent: Jens Niemeyer, Institut für Astrophysik, Universität Göttingen

Koreferent: Dominik Schleicher, Institut für Astrophysik, Universität Göttingen

Weitere Mitglieder der Prüfungskommission:

Wolfram Kollatschny, Institut für Astrophysik, Universität Göttingen

Ansgar Reiners, Institut für Astrophysik, Universität Göttingen

Stefan Dreizler, Institut für Astrophysik, Universität Göttingen

Wolfram Schmidt, Institut für Astrophysik, Universität Göttingen

Tag der mündlichen Prüfung: 


\section{Contents}

1 Introduction $\quad 1$

1.1 Observations of LAEs . . . . . . . . . . . . . . . . . . . . 3

1.2 Observational Properties of LAEs . . . . . . . . . . . . . . . 4

1.3 Lyman-Alpha Radiative Transport . . . . . . . . . . . . . . . . 5

1.3.1 Radiative Transfer Algorithm for the Lyman-Alpha Line . . . . 10

1.3.2 Adaptive Mesh Refinement (AMR) . . . . . . . . . . . . . . . 11

1.3.3 Additional Physics for Lyman-Alpha Transport in Cosmological

Simulations ..................... 12

1.3.4 The Treatment of Continuum Photons . . . . . . . . . . . . . 12

1.3.5 Speeding Up Calculations and Parallelization . . . . . . . . . . . 13

1.3.6 Used Implementations of the Radiative Transport . . . . . . . . 13

2 Large-Scale Structure Surveys and the Influence of Lyman-Alpha $\begin{array}{ll}\text { Radiation Transport } & 15\end{array}$

2.1 Contamination by Attenuation in the Large-Scale Structure . . . . . . 19

2.2 Contamination from Anisotropic Escape and Tidal Alignment . . . . . 30

3 State of the Art: LAE Modeling $\quad 35$

3.1 Isotropic Simplified Models . . . . . . . . . . . . . . . . . 35

3.2 Anisotropic Simplified Models . . . . . . . . . . . . . . . 43

3.3 Towards Realistic LAEs . . . . . . . . . . . . . . . . . . . . . . 51

3.3.1 Models Based on Cosmological Simulations . . . . . . . . . . . . 51

3.3.2 Models Based on Simulations of Isolated Galaxies . . . . . . . . 55 
4 Accepted Publications

4.1 Effects of Lyman-Alpha Scattering in the IGM on Clustering Statistics of Lyman-Alpha Emitters . . . . . . . . . . . . . . . . . . . . 63

4.2 Beamed Ly $\alpha$ Emission through Outflow-driven Cavities . . . . . . . . 77

4.3 Inclination Dependence of Lyman- $\alpha$ Properties in a Turbulent Disk Galaxy ........................ 89

$\begin{array}{llr}5 & \text { Summary and Discussion } & 99\end{array}$

$\begin{array}{ll}\text { Bibliography } & 105\end{array}$

$\begin{array}{ll}\text { Appendix } & 125\end{array}$

A Contributions to the Publications $\quad \mathbf{1 2 5}$

A.1 Effects of Lyman-Alpha Ccattering in the IGM on Clustering Statistics of Lyman-Alpha Emitters . . . . . . . . . . . . . . . . . . . . 125

A.2 Beamed Ly $\alpha$ Emission through Outflow-driven Cavities . . . . . . . . 125

A.3 Inclination Dependence of Lyman- $\alpha$ Properties in a Turbulent Disk Galaxy . . . . . . . . . . . . . . . . 126

B A Non-exhaustive List of LAE Detections $\quad 127$

$\begin{array}{ll}\text { Publications } & 131\end{array}$

$\begin{array}{ll}\text { Curriculum Vitae } & 133\end{array}$ 

The effort to understand the universe is one of the very few things which lifts human life a little above the level of farce and gives it some of the grace of tragedy.

Steven Weinberg, The First Three Minutes

\section{CHAPTER 1}

\section{Introduction}

Since the realization that the Milky Way is only one of myriads of galaxies in the aftermath of the "Great Debate", the study of galaxies has become a vital field in astronomy. While matters of galaxy formation, galaxy evolution, star formation, and other processes in galaxies are intrinsically important, it is interesting to notice that the study of galaxies has a wider application in cosmology, because galaxies are thought to trace the large-scale structure of the Universe, yielding insights on the large-scale matter distribution, cosmological parameters, and structure formation as a cosmological process. Moreover, galaxies are a unique tool for cosmology in the sense that they are typically the only detectable elements of the large-scale structure.

Naturally, studying galaxies at large distances, i.e. high redshifts, is a difficult task due to their low apparent luminosity. In the last decade, the systematic search for high-redshift galaxies has been supplemented by techniques for the detection of Lyman- $\alpha$ emission from such objects. Galaxies that are detected primarily by their Lyman- $\alpha$ emission are therefore called Lyman- $\alpha$ emitters (LAEs).

The Lyman- $\alpha$ line, originating from the $(n=2 \Rightarrow n=1)$ electronic transition of a hydrogen atom $\left(\lambda_{0}=1215 \AA\right)$, is by far the most prominent emission line in star-forming galaxies (Laursen 2010). The main mechanism for generating Lyman- $\alpha$ emission is thought to be recombination of hydrogen after ionization by energetic radiation from young stars, supernovae, QSOs, or UV background. Additionally, cooling radiation from gas collapsing into the potential well of a dark matter halo is considered a possibly important source at high redshifts (e.g Dijkstra et al. 2006).

While being hard to observe with ground-based telescopes at low redshift $(z<1.5)$ due to geocoronal emission, the redshifted Lyman- $\alpha$ line is easily observable at 
higher redshifts (Barnes et al. 2014). The potential of the Lyman- $\alpha$ line was already discovered about 40 years ago (Partridge \& Peebles 1967, also see section 1.1), although successful observational campaigns are not older than 25 years (see section 1.1).

More than being an efficient tool to detect high-redshift galaxies, the population of LAEs itself presents a valuable object of investigation. As will be discussed in section 1.2, LAEs are typically star-forming galaxies in one of their first episodes of intense star formation and provide therefore information on processes of galaxy formation. However, LAEs do not form necessarily a distinct class of physically similar objects the LAE sample is merely the collection of the objects detected in Lyman- $\alpha$ emission. This is also illustrated by the fact that the observed properties of LAEs show large variations, e.g. in terms of their spatial extent, masses, and spectral properties.

The observational properties of LAEs pose a big challenge for interpretation due to the fact that the Lyman- $\alpha$ transition is a resonant line - even small amounts of neutral hydrogen along the path of a Lyman- $\alpha$ photon will induce many scatterings. Additionally, velocity fields, dust content, and the thermal state of the environment play a large role in the transport of Lyman- $\alpha$ photons. Since the radiative transfer is in general too complex for analytical treatment, a number of authors have started the endeavor of studying the radiative transfer of Lyman- $\alpha$ photons in/around their sources numerically. Again, this small field has become vital about 10 years ago, since numerical studies have only become computationally feasible in the last decade. Despite the fact that both on the numerical and on the observational side progress has been made, many open questions remain. The numerial work on LAEs has largely focused on simplified geometries with spherical symmetry, and there are few publications on detailed, realistic simulations of Lyman- $\alpha$ transport in galaxies. Additionally, scheduled large-scale surveys like HETDEX ${ }^{1}$ that rely on LAEs to probe the large-scale structure demand for a thorough understanding of the properties of LAEs to avoid systematic errors introduced by the complicated radiative transfer of Lyman- $\alpha$ photons.

This thesis is devoted to improving our understanding of the radiative transfer in LAEs, and the connection between observed properties of LAEs and their large-scale surroundings. It will therefore primarily be concerned with a small subset of open questions in the field of LAEs:

1 Hobby Eberly Telescope Dark Energy Experiment 
- What is the influence of anisotropies in the gas distribution and kinematics on observed LAE properties?

- To which extent do correlations of large-scale structure and LAE properties contaminate large-scale surveys utilizing LAEs as tracers of the large-scale matter distribution?

- What can we learn from simplified, anisotropic models of LAEs?

These three questions are in fact connected with each other, since as will be shown in section 2.2 , the local, small-scale anisotropy in density, e.g. simply induced by a disky geometry, can lead to significant contamination of large-scale surveys under certain circumstances. This means that anisotropy of LAEs can in fact contaminate measurements on the large scales probed by surveys.

The outline of this thesis is the following: In the next sections, we will give a brief summary of observations of LAEs (section 1.1) and the observed properties of LAEs (section 1.2). In section 1.3, we will summarize the physics of Lyman- $\alpha$ transport and the implementation of the transport in a Monte-Carlo code. Chapter 2 will lay down the theoretical background for considering possible contamination of large-scale surveys by radiative transfer effects in LAEs, with the first section (2.1) focusing on the large-scale environment affecting Lyman- $\alpha$ properties, and the second section (2.2) devoted to the possibility of contamination by tidal alignment of galactic disks and anisotropic escape. Chapter 3 will briefly review the state of the art of LAE modeling, in particular modeling of the interstellar medium (ISM) of individual emitters. After investigating the work done on simplified isotropic models in the literature (section 3.1), we turn to anisotropic extensions (section 3.2) and finally towards more realistic models (section 3.3). In chapter 4, we present the three accepted publications that were compiled during the time of the author's $\mathrm{PhD}$ program. Finally, we summarize and discuss our main results in chapter 5 .

\subsection{Observations of LAEs}

We proceed to give a brief overview of observational aspects of Lyman- $\alpha$ emission, beginning with a short historical excursion. The possibility of using Lyman- $\alpha$ emission to find distant galaxies was already suggested by Partridge \& Peebles 1967. Their optimistic estimation led them to the conclusion that forming, young galaxies could have a Lyman- $\alpha$ line flux of about $10^{44} \mathrm{erg} / \mathrm{s}$. For about thirty years, these predictions could not be verified in observations, despite numerous surveys that tried 
to detect such objects (for a review, see Pritchet 1994), although a few individual objects were found by targeted spectroscopy (see Djorgovski et al. 1985, for example). However, beginning in the nineties of the last century, more and more LAEs were detected in both narrow band searches and spectroscopic surveys (e.g. Hu et al. 1998; Pascarelle et al. 1998; Rhoads et al. 2001; Steidel et al. 2000, among others). The main reason for the unsuccessful search for LAEs is that the typical line flux from these objects has been found to be about $10^{42} \mathrm{erg} / \mathrm{s}$, two magnitudes lower than expected. It is assumed that this severe discrepancy between the original expectation of Partridge \& Peebles 1967 and the observational findings is primarily due to the interplay of resonant scattering and dust attenuation, and incorrect cosmological assumptions made by Partridge \& Peebles 1967 (Barnes et al. 2014, also see chapter 2).

Since the beginning of the new millennium, large surveys have detected of the order of $10^{3}$ LAEs (see table B.1 for details) at redshifts between 1 and 7 . At redshifts $>6.5$, the Lyman- $\alpha$ line is subject to considerable absorption from the neutral intergalactic medium (IGM), rendering detections difficult (e.g. Tilvi et al. 2010). The most prominent technique for the detection of LAEs is a combination of narrow band imaging and continuum band imaging. Frequently, a spectroscopic follow-up observation is used to rule out contamination from low-redshift [OII], [OIII], and $\mathrm{H} \alpha$ emitters.

Future instruments will improve our knowledge of LAEs in terms of number counts, depth, and resolution. In particular, the HETDEX survey will increase the number of detected LAEs by about 800.000. Subaru Hyper Suprime Cam and MUSE ${ }^{1}$ will deliver both high-redshift emitters with unprecedented flux limits and improve spectral and spatial resolution of individual emitters: For example, MUSE will reach a velocity resolution of tens of $\mathrm{km} / \mathrm{s}$ at $z \sim 3-4$ (Henault et al. 2003). Finally, the JWST $^{2}$ will perhaps allow to detect galaxies at redshifts up to 10 (Dijkstra 2014; Dijkstra et al. 2011).

\subsection{Observational Properties of LAEs}

LAEs are found to be typically young systems with ages of 10 Myr to 1 Gyr (Gawiser et al. 2007; Lai et al. 2008). Their stellar mass is of the order $10^{7.5}-10^{10.5} \mathrm{M}_{\odot}$ (e.g.

1 Multi Unit Spectroscopic Explorer

2 James Webb Space Telescope 
Gawiser et al. 2007; Hagen et al. 2014), and their star formation rate is typically found to lie between 1 and $100 \mathrm{M}_{\odot} \mathrm{yr}^{-1}$ (Gawiser et al. 2007; Gronwall et al. 2007; Hagen et al. 2014). They are typically dust-free $(\mathrm{E}(\mathrm{B}-\mathrm{V})<0.1)$ (Gronwall et al. 2007; Hagen et al. 2014), although objects with high dust content have also been found (up to $\mathrm{E}(\mathrm{B}-\mathrm{V})=0.4$ ) (e.g. Hagen et al. 2014). As Hagen et al. 2014 point out, objects with high dust content do not show signs of resonant scattering, i.e. the Lyman- $\alpha$ flux escapes the object unscattered, which is consistent with the picture that dust will absorb Lyman- $\alpha$ radiation if resonant scattering is efficient. With respect to size, LAEs appear to be compact objects, with half-light radii of about $\sim 1 \mathrm{kpc}$, but some objects reach up to a few kpc (Bond et al. 2012; Hagen et al. 2014). Concerning the clustering bias that has been measured at redshift 2-3, LAEs are typically observed to reside in halos of $10^{11.5} \mathrm{M}_{\odot}$ with a bias factor of $\approx 1.8$ (Guaita et al. 2010). As pointed out by several authors, this makes LAEs the 'building blocks' of $\mathrm{L}^{*}$ galaxies at $z=0$ (e.g. Guaita et al. 2010).

While the scatter in Lyman- $\alpha$ properties is large, the 'average' LAE is young $(<$ $100 \mathrm{Myr})$, dust-free, small $(<1 \mathrm{kpc})$ and light-weighted $\left(\sim 10^{8} \mathrm{M}_{\odot}\right.$ in stellar mass $)$, having star formation rates of $1-10 \mathrm{M}_{\odot} \mathrm{yr}^{-1}$ and very high specific star formation rate, i.e. a high star formation rate relative to their stellar mass (e.g. Laursen 2010).

A special subclass of LAEs are the so-called Lyman- $\alpha$ blobs, which are extended LAEs with sizes of up to $100 \mathrm{kpc}$ (e.g Matsuda et al. 2011). Their physical origin is unclear: Possible scenarios can be divided into those assuming central sources and those assuming an extended source for the Lyman- $\alpha$ emission. Assuming central sources, massive galaxies undergoing large starbursts and AGNs are candidates for the origin of the blobs (Cen \& Zheng 2013; Hayes et al. 2011 but also see Tamura et al. 2013), while gravitational cooling from gas falling into a massive halo could be the source if one assumes an extended emission origin (e.g. Matsuda et al. 2011).

\subsection{Lyman-Alpha Radiative Transport}

As has been already noted above, there are two main sources of Lyman- $\alpha$ photons. On the one hand, recombination after ionization by hard UV radiation converts UV continuum radiation efficiently into Lyman- $\alpha$ photons:

$$
e^{-}+\mathrm{H}^{+} \longrightarrow \mathrm{H}\left(1^{2} \mathrm{~S}\right)+\gamma_{L y \alpha}+\gamma
$$


The reason is that recombining hydrogen typically goes into the ground state via a cascade of transitions due to quantum-physical restrictions on possible transitions. If one assumes that the ionizing photon that is emitted if the atom recombines directly into the ground state, is immediately reprocessed (i.e. the environment is optically thick to ionizing flux), emission of a Lyman- $\alpha$ photon follows in $68 \%$ of all cases, i.e. $68 \%$ of the energy of the ionizing radiation is converted into Lyman- $\alpha$ (Barnes et al. 2014). The sources of the ionizing radiation that is converted are thought to be massive stars, supernovae, AGNs, or the UV background (in especially, the local UV background can be enhanced in the proximity of a nearby QSO (e.g. Cantalupo et al. 2014)). Stars are typically assumed to be the dominant source of the converted ionizing radiation (Laursen et al. 2009). Since the massive O and B stars that produce the necessary flux in the UV have a short life time of $\sim 10^{6}-10^{7} \mathrm{yr}$, the production of Lyman- $\alpha$ photons is strongly coupled to the recent star formation activity of a galaxy.

On the other hand, collisional excitation can lead to emission of Lyman- $\alpha$ photons. According to many studies on structure formation, young galaxies should partly accrete their matter in form of cold streams (e.g. Birnboim \& Dekel 2003), streams of cold gas falling into the center of the dark matter halo. These cold streams are not shock-heated near the virial radius, and are thought to cool via Lyman- $\alpha$ radiation. As Dijkstra et al. 2006 point out, such emission would be typically extended and blue-shifted, rendering a detection at high redshift improbable due to attenuation by the IGM (also see chapter 3). Additionally, they note that this source of Lyman- $\alpha$ radiation is not well understood, i.e. it is not known where in the cold streams conditions are sufficient to generate significant amounts of Lyman- $\alpha$ radiation.

We proceed to briefly review how radiative transport of Lyman- $\alpha$ photons is described. A more detailed description can be found in Behrens 2011; Dijkstra et al. 2006; Laursen 2010; Verhamme et al. 2006; Zheng \& Miralda-Escude 2002. Here, we mainly summarize the presentation of Behrens 2011, which is based on Dijkstra et al. 2006; Verhamme et al. 2006 and Laursen 2010.

Lyman- $\alpha$ photons scatter resonantly on neutral hydrogen. The probability $P$ that a photons is scattered along a path $p$ in a medium of neutral gas with number density $n$ is written as

$$
P=e^{-\tau}
$$


$\tau$ is called the optical depth, and if only hydrogen contributes to the optical depth, we can write

$$
\tau=\tau_{H}=\int_{p} \sigma_{\nu}(x) n(\vec{r}) d \vec{r}
$$

Here, $\sigma_{\nu}(x)$ denotes the scattering cross section that depends on the frequency $x$ of the photon. We write the physical frequency $\nu$ as a dimensionless quantity:

$$
x=\frac{\nu-\nu_{0}}{\nu_{D}}
$$

Here, $\nu_{D}=\frac{v_{t h} \nu_{0}}{c}$ is the Doppler frequency with $v_{t h}$ the typical thermal velocity of the gas, $\nu_{0}$ the line center frequency of the Lyman- $\alpha$ line, and $c$ the speed of light. Intuitively, the quantity $x \nu_{0} / c$ measures the frequency shift from the line center in units of the thermal velocity of the gas. In the case of a non-thermal contribution to the Doppler frequency, e.g. if a RMS turbulent velocity $v_{\text {turb }}$ is present, we can modify $\nu_{D}$ by quadratically adding the turbulent and thermal contributions:

$$
\nu_{D}=\frac{\sqrt{v_{t h}^{2}+v_{t u r b}^{2}} \nu_{0}}{c}
$$

The cross section $\sigma_{\nu}(x)$ can be written as:

$$
\sigma_{\nu}(x)=\frac{f_{12} \sqrt{\pi} e^{2}}{m_{e} c \nu_{D}} H(a, x)
$$

with $e$ the charge of the electron, $m_{e}$ its mass, and $f_{12}$ the so-called oscillator strength of the Lyman- $\alpha$ transition. $H(a, x)$ is the so-called Voigt-Profile defined as:

$$
H(a, x)=\frac{a}{\pi} \int_{-\infty}^{\infty} \frac{e^{-y^{2}}}{(x-y)^{2}+a^{2}} d y
$$

Here, $a$ is the Voigt parameter related to the line width of the Lyman- $\alpha$ line. The Voigt-Profile results from the folding of a Lorentz profile for the natural line width of the line with the thermal distribution of the gas atoms on which the photons scatter. It is frequently approximated by an exponential $e^{-x^{2}}$ in the line center (for $|x|$ smaller than a certain value, e.g. $2.5>|x|$ in Dijkstra et al. 2006) and with $\frac{a}{\sqrt{\pi} x^{2}}$ far away from the line center.

In general, the gas atoms will also have a non-zero bulk velocity. We assume that $x$ 
denotes the frequency in the observer's frame. We can use a Lorentz transformation to evaluate the frequency of the photon $x^{\prime}$ in the rest frame of a gas atom with velocity $\vec{v}_{A}$ :

$$
x^{\prime}=x-\frac{\vec{v}_{A} \vec{n}}{v_{t h}}
$$

Here $\vec{n}$ is the direction of the infalling photon.

When a scattering occurs, the frequency of the photon in the observer frame is changed in general because of the non-zero velocity of the scattering atom. In the rest frame of the atom, we denote the frequency of the infalling photon with $x_{i}^{\prime}$ and the frequency after scattering with $x_{o}^{\prime}$. Due to coherence, $x_{o}^{\prime}=x_{i}^{\prime}$. We can evaluate the frequency of the scattered photon in the observer's frame $x_{o}$ by applying eq. 1.8 twice to first switch into the rest frame of the atom, and then go back to the observer's frame:

$$
x_{o}=x_{i}+\frac{\vec{v}_{A}\left(\vec{n}_{o}-\vec{n}_{i}\right)}{v_{t h}}
$$

We see that the frequency stays constant only in cases where $\vec{n}_{i}=\vec{n}_{o}$ or $\vec{v}_{A}=\overrightarrow{0}$, i.e. when the the photons undergoes no change in direction and/or when the atom's velocity is zero. We neglected here the recoil of the scattering on the atom since it has been shown to be negligible except for extremely low temperatures of the gas (Zheng \& Miralda-Escude 2002).

To evaluate these equations, one needs to know the probability distribution of the velocities of scattering atoms. The velocity vector can be split into the component parallel to the direction of the infalling photon $\vec{u}_{\|}$and two orthogonal components $\vec{u}_{\perp, 1} / \vec{u}_{\perp, 2}$. While the orthogonal components are just thermally distributed according to the usual Maxwell distribution, the parallel component is a Maxwell distribution folded with the Lorentzian profile of the line. Consequently, the probability for a photon far away from the line center to be scattered is highest for an atom that has a component $u_{\|}$so that in the rest frame of the atom, the photon appears near the line center. On the other hand, at large $u_{\|}$the number of atoms becomes very low due to their thermal distribution, making scattering at lower-velocity atoms again more probable although in the restframe of these atoms, the photon is far away from 
the line center. The resulting probability distribution for $u_{\|}$can be written as

$$
f\left(u_{\|}\right)=\frac{a}{\pi H(a, x)} \frac{e^{u_{\|}}}{\left(x-u_{\|}\right)^{2}+a^{2}}
$$

To evaluate eq. 1.9, we need to find a connection between the direction of the incoming and the outgoing photon. This is given by the so-called phase function. Although quantum theoretical considerations show that the choice of the correct phase function depends on the frequency of the infalling photon (Tasitsiomi 2006), in practice the probability distribution of outgoing directions is often chosen to be a dipole and be can be written as a function of one angle $\Theta$ because of the symmetry of the problem:

$$
P(\Theta)=1+\frac{3}{7} \cos ^{2} \Theta
$$

This concludes the basic summary the physics of Lyman- $\alpha$ scattering on neutral hydrogen.

In principle, the optical depth for a Lyman- $\alpha$ photon also has contributions from other lines, e.g. the corresponding deuterium line (see for example Dijkstra et al. 2006) or dust. We discuss here how dust can be included, but the procedure is the same for other contributions. In the case of dust, the only complication is that besides scattering, a second physical interaction with Lyman- $\alpha$ photons can occur, namely absorption. The optical depth due to dust can be written as

$$
\tau_{D}=\int^{p} \sigma_{D} n_{D}(\vec{r}) d \vec{r}
$$

similar to eq. 1.3. $n_{D}$ is the dust grain density, $\sigma_{D}$ is the interaction cross section. Following (Verhamme et al. 2006), we can write the interaction cross section as

$$
\sigma_{D}=\pi d^{2}\left(Q_{a}+Q_{s}\right)
$$

with $d$ the typical grain diameter and $Q_{a}\left(Q_{s}\right)$ the absorption (scattering) efficiency.

The total optical depth a photon encounters is then the sum of the contributions from dust and hydrogen,

$$
\tau=\tau_{H}+\tau_{D}
$$


When interacting with a dust particle, Lyman- $\alpha$ photons can be absorbed with subsequent emission of infrared photons by the grain. From the point of view of Lyman- $\alpha$ transport, these photons are 'lost' or destroyed since they are reemitted far away from the line center. The albedo $A=\frac{Q_{S}}{Q_{A}+Q_{S}}$ is the probability for the photons to be scattered, and $1-A$ is the probability for absorption. For UV wavelengths, a value of $A=0.5$ for the albedo can be adopted since absorption and scattering are approximately equally likely (Verhamme et al. 2006, e.g.). Finally, a phase function has to be chosen for the case of scattering on dust. A Greenstein phase function can be used to fit dust observations in our Galaxy (Henyey \& Greenstein 1941):

$$
P(\Theta)=\frac{1}{4 \pi} \frac{1-g^{2}}{\left[1+g^{2}-2 g \cos \Theta\right]^{3 / 2}}
$$

Here, $g$ is the so-called anisotropy factor. A value of zero corresponds to isotropic scattering, while values of 1 (-1) lead to complete forward (backward) scattering. In the literature, values of $g \sim 0.7$ are chosen, derived from observations (Inoue 2003). This indicates a tendency of dust grains to be predominantly forward-scattering.

\subsubsection{Radiative Transfer Algorithm for the Lyman-Alpha Line}

In this section, we briefly present the typical algorithm for following the radiative transfer of Lyman- $\alpha$ photons in astrophysical contexts. Starting with Ahn et al. 2000, the Monte-Carlo approach has been widely used for this purpose. Given density, temperature, and velocity fields, one sets up a number of tracer photons with an initial position, frequency, and direction. Then, for each photon, one proceeds with the following algorithm that we depict here for the case of a dust-free medium:

1. Draw a random, exponentially distributed optical depth $\tau_{I}$

2. Move the photon according to its direction vector, integrate the optical depth $\tau$ along the way

3. If $\tau_{I}=\tau$, an interaction with the gas will happen

a) Draw a parallel velocity component for the scattering atom according to the distribution in eq. 1.10. Draw the orthogonal components from a Maxwell distribution

b) Draw an outgoing direction vector from the unit sphere according to the given phase function

c) Evaluate the frequency of the photon after the scattering using eq. 1.8 
4. Repeat until the photon has left the simulation domain

The properties of all photons that leave the domain are stored, e.g. their frequency in the observer frame, their direction and the number of scatterings undergone.

If dust is present, one has to decide if the photon interacts (item 3. in the list above) with either a gas atom or a dust particle. For this purpose, one can draw a random number with flat distribution, $0<R<1$. If $R<\tau_{D} / \tau$, an interaction with dust occurs. Other contributions can be added accordingly. In the case of an interaction with dust, we have to decide whether the process of interaction is either absorption or coherent scattering of the photon. We can again draw a random number with flat distribution, $0<R_{2}<1$, scattering occurs according to eq. 1.13 and 1.15 if $R_{2}<A$, else absorption occurs.

As expected for a Monte-Carlo method, it is crucial to follow a sufficient number of photons to get reliable results. This can be computationally challenging, mainly because of the calculation needed to obtain the parallel velocity component of a scattering atom (eq. 1.10). This probability distribution is not invertible analytically and the so-called rejection method (see Press et al. 2007, section 7.3.6) is needed to invert it which is numerically a very expensive operation.

The emissivity of Lyman- $\alpha$ photons in such simulations can be set assuming a list of point sources. In particular, this is convenient if the emitting region is not spatially resolved in the simulation. At smaller scales and higher resolution, the emissivity can be given by an additional field, attributing an intrinsic emissivity to every cell in the simulation volume. In the following, we assume the radiative transport simulation to be run on a static configuration of density/velocity/temperature/dust as a post-processing step. This means that these fields do not evolve in time during the transport simulation. This is based on the assumption that the timescale of the escape of Lyman- $\alpha$ photons is much shorter than the dynamical timescale.

\subsubsection{Adaptive Mesh Refinement (AMR)}

Grid-based simulations of astrophysical objects are frequently not done on a single, uniform grid, but feature a hierarchy of grids with varying cell sizes. They typically form a hierarchy in the sense that a coarser grid might be refined partially by a finer grid. In this way, one can achieve a very high resolution in regions that contain the relevant physical objects and processes, while keeping the resolution (and therefore: the computational costs) low in other regions. The radiative transfer codes used for the work presented here can use this technique. From the computational point of 
view, only the routines integrating the optical depth are modified to use multiple grids. While in a uniform grid, one can simply integrate the optical depth of the photons' path through each cell, one has to check for every integration step whether or not the photon has just left or entered a refined region of the simulation volume when using AMR grids.

\subsubsection{Additional Physics for Lyman-Alpha Transport in Cosmological Simulations}

To simulate Lyman- $\alpha$ radiation in a cosmological simulation, at least two requirements have to be fulfilled numerically. On the one hand, there is a physical process that has to be modeled numerically, namely the Hubble expansion that leads to redshifting of photons. This can be done by redshifting photons between scatterings according to

$$
\delta z=\frac{H(z) d}{c}
$$

with $H(z)$ the Hubble rate at the mean redshift of the simulation $z$ and the distance $d$ traveled by the photon since the last scattering (e.g. Behrens \& Niemeyer 2013). This approach is valid as long as the evolution of $H(z)$ within the simulation volume is small.

On the other hand, cosmological volumes are typically simulated using periodic boundaries, i.e. if the simulation box has a volume $l^{3}$, the cell at a position $(x, y, z)$ is identical to the cells at $(x+i l, y+j l, z+k l)$, where $i, j$, and $k$ are integers. To remove artifacts arising at the boundaries, one has to apply these periodic boundaries as well for the Lyman- $\alpha$ photons. Here, the question arises how to decide when the radiative transfer of an individual photon is assumed to be complete (that is, when a photon is considered to reach the observer without further scatterings), since due to periodic boundaries, the simulation domain is now infinite in extent. One possibility here is to define a cut-off distance. If the distance between the photon and the region it originated from is larger than this cut-off distance, the transfer is assumed to reach the observer without further scatterings (used by e.g. Behrens \& Niemeyer 2013; Zheng et al. 2010). The assumption is physically justified if the cut-off distance is chosen large enough so that the Hubble expansion has effectively shifted the photon out of resonance.

\subsubsection{The Treatment of Continuum Photons}

In several contexts, it is important to not only follow the propagation of Lyman- $\alpha$ photons, but also continuum radiation. A simple approach here for continuum 
radiation in the UV is to run the same algorithm for these photons as for the Lyman$\alpha$ photons, since the Lyman- $\alpha$ line is the most important contributor to the optical depth for these photons as well (e.g. Verhamme et al. 2006).

\subsubsection{Speeding Up Calculations and Parallelization}

As has already been mentioned, simulations of Lyman- $\alpha$ radiative transfer are computationally demanding. To speed up calculations, one strategy is to avoid so-called core scatterings (Dijkstra et al. 2006). Most scatterings of a Lyman- $\alpha$ photon in the line center do not change its frequency significantly. In an optically thick environment, this means that Lyman- $\alpha$ photons may scatter thousands of times without moving significantly in frequency or space. In those environments, these core scatterings can be avoided by forcing scatterings of photons in the line center to occur on high-velocity atoms. In these scatterings, the photons will typically be scattered out of the line center. For photons not in the line center and/or for photons in optically thin environments, this suppression leads to artifacts and should be avoided. In the other cases, it is justified to suppress core scatterings by simply 'cutting off' the Maxwellian distribution of the velocity components of the scattering atoms that are perpendicular to the infalling photon. The desired random velocity components can be generated via:

$$
u_{\perp} \propto \sqrt{x_{c}^{2}-\ln R_{1}} \cos 2 \pi R_{2}
$$

where $R_{1}$ and $R_{2}$ are again random number with flat distribution between 0 and 1 . $x_{c}$ parametrizes the cut-off. As Laursen et al. 2009 point out, $x_{c}$ is a function of the local optical depth and the frequency of the infalling photon. Their formula for calculating $x_{c}$ is also implemented in the code(s) the author developed and used for the publications presented in chapter 4 .

Another important way of speeding up the calculations is parallelization, i.e. using multiple processors at the same time. The code(s) developed in the course of this thesis are both MPI- and OpenMP parallelized, i.e. they use both multiple threads to parallelize work in a single process (a single instance of a program) and multiple processes.

\subsubsection{Used Implementations of the Radiative Transport}

For the work presented in this thesis, two implementations were used to follow the transport of Lyman- $\alpha$ photons, named LyS and Sedona. LyS was used in Behrens \& 
Niemeyer 2013, while Sedona was used in Behrens et al. 2014 and Behrens \& Braun 2014. Both are based on $\mathrm{C}++$. While LyS was built from scratch and designed for use with Enzo and Ramses grid (AMR) structures, Sedona was developed on the basis of the library BoxLib to handle Nyx' (Almgren et al. 2013) (AMR) grid structure. The main reason for switching codes was the need to run simulations done by Nyx, and the fact that a prototype of a radiative transfer code was available from the developers of Nyx, i.e. a piece of code containing nothing but the computational infrastructure for such a code, but without any relevant physics. Developing a new Lyman- $\alpha$ code on this base was considered simpler than integrating the Nyx/BoxLib infrastructure into LyS. Additionally, this also has the advantage that we were able to use the built-in parallelization of BoxLib. While the Sedona code used in Behrens et al. 2014 and in Behrens \& Braun 2014 is parallelized by virtue of the underlying BoxLib library, but needed minor modifications, the code LyS that was used in Behrens \& Niemeyer 2013 was parallelized completely by hand.

LyS implemented a basic Lyman- $\alpha$ transport scheme, emission from a list of point sources, isotropic/dipole phase functions, the acceleration scheme explained in section 1.3.5, handling of redshifting due to the Hubble flow, and the handling of periodic boundaries with a fixed cut-off distance (see section 1.3.3).

Sedona has the same features (except for the Hubble flow handling and the periodic boundaries), but includes the capability to account for dust, with a Greenstein phase function for the anisotropic scattering on dust (see equation 1.15). It also allows to follow the transport of continuum photons near the Lyman- $\alpha$ line. Emission of photons can come from point sources and/or by an emissivity field in the simulation domain. Sedona also has a 'scripting' capability: Simple distributions of gas and dust can be set up using a simple text file instead of editing the code of the program. Linear or radial velocity fields can also be added in the same way. This was used for the simple models of LAEs in Behrens et al. 2014. 


\section{CHAPTER 2}

\section{Large-Scale Structure Surveys and the Influence of Lyman-Alpha Radiation Transport}

The fact that the Lyman- $\alpha$ line is the most powerful emission line in star-forming galaxies makes it a potential tool for observations of the large scale structure of the Universe by mapping out a large number of LAEs and use them to reconstruct the matter distribution. Since the atmosphere is transparent for Lyman- $\alpha$ radiation at redshift above $\sim 1.5$ and the star-formation history peaks somewhere around $z \sim 2$ this is feasible even with ground-based telescopes, reducing the costs of such an endeavor which is currently undertaken by the HETDEX collaboration (Hill et al. 2008).

The resonant scattering of Lyman- $\alpha$ photons on their way from their source to an observer can modify observed properties of LAEs in a non-trivial way. One important question with respect to large-scale surveys is therefore if and how the transport within a galaxy and in the environment correlates the observed Lyman- $\alpha$ properties like flux and spectra with e.g. the kinematics and gas/dust distribution of the emitter and its surroundings. In principle, these correlations could spoil a measurement of the large-scale distribution of matter by, for example, systematically shifting the flux of emitters in certain environments below the detection threshold. Therefore, it is crucial to understand how important these effects are and how they could potentially be corrected for. To motivate this problem in more detail, we will turn to a simple toy model.

Lyman- $\alpha$ radiation originating from star-forming galaxies or other sources of ionizing radiation is, due to the resonant nature of the line, scattered by neutral hydrogen even at low densities. The photons are mainly affected by the spatial 
density and velocity structure: The probability of interaction along the path of a photon, $P_{I}=1-e^{-\tau}$, exponentially goes to 1 with both the density $(\tau \propto \rho)$ and the cross section $(\tau \propto \sigma)$, and the cross section is exponentially reduced by a bulk velocity shift of the intervening gas ${ }^{1}$. For example, for a gas at a temperature $T=1 \times 10^{3} \mathrm{~K}$, the optical depth is reduced by a factor of $\approx 5$ if the gas has a bulk velocity as small as $8 \mathrm{~km} / \mathrm{s}$ parallel to the direction of the infalling photon (for a Lyman- $\alpha$ photon that is in the line center). Anisotropies and inhomogeneities in the density and in the velocity fields are therefore imprinted onto the escaping Lyman- $\alpha$ radiation.

This can be illustrated easily by considering a long slab of gas with a central, isotropic point source of Lyman- $\alpha$ radiation in its center, as depicted in Fig. 2.1. We assume for now that the slab is optically thick, so that along the short axis $a$ of the slab, the optical depth is about $10^{6}$ from the center to the edge, while it is $10^{7}$ along the long axis $b$, and that the Lyman- $\alpha$ photons are isotropically emitted at line center frequency. In this case, photons will undergo many scatterings $\left(\sim 10^{6}\right)$ before leaving the slab. Scattering shuffles their directions, and may shift their frequency out of the line center until they can escape. Since axis $a$ is ten times shorter than $b$, the probability for a photon to escape in the direction of axis $b$ is reduced, because it is more probable for them to diffuse out of the slab along the $a$ axis. The flux as a function of the inclination $|\cos \theta|=\vec{a} \vec{k}$, where $\vec{k}$ is the direction of the escaping photon, is shown in Fig. 2.2. We see that indeed the flux is clearly peaked along the short axis.

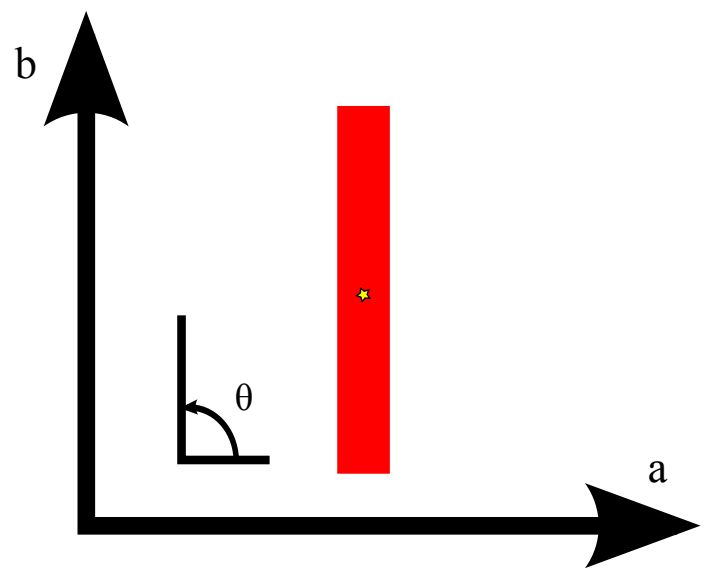

Figure 2.1: Illustration of a slab of gas with a central source.

1 This is true close to the line center, see 1.3 


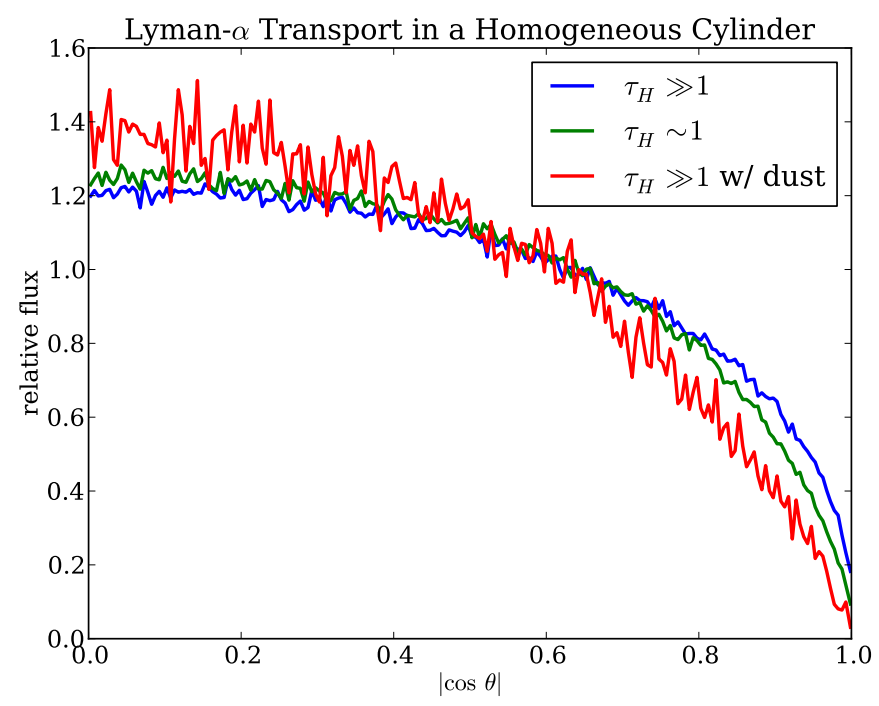

Figure 2.2: Lyman- $\alpha$ flux as a function of inclination for a homogeneous slab with an axis ratio of 10 for three cases: optically thick (blue), optically thin (green), optically thick with additional dust content (blue) $\left(\tau_{D} \sim 1\right)$. The scatter in the latter plot comes from the fact that only $6 \%$ of the photons escape the slab in this case.

If we assume the optical depth to be much lower, as it might be the case in the ionized intergalactic medium, and set the optical depth along axis $a$ to $\tau=1$, we still observe the flux to be peaked towards the short axis. While in the case of a large optical depth, photons mainly diffuse in real space and frequency due to many scatterings, in the case of low optical depth, only few scatterings occur. In this case, we can approximate the fraction of photons that escape in a certain direction by the fraction of photons $F$ that were emitted in that direction and actually were transmitted in that direction:

$$
F=e^{-\tau}
$$

This means that we assume that a photon is not scattered back into its initial direction by subsequent scatterings; every scattering 'removes' the photon out of this line of sight. Since $F$ is larger for directions along axis $a$, the flux is relatively enhanced. This so-called $e^{-\tau}$-approach does not apply in optically-thick regions in general, because for a photon that is scattered out of the line of sight, there is a non-vanishing probability to get back into the line of sight after numerous scatterings in these regions (e.g. Laursen et al. 2010). 
So far, we have only introduced an anisotropic density distribution: Adding an anisotropic velocity field would also affect the optical depth severely, with the additional complication that in this case, the local optical depth also depends on the direction of the photon. While the spectra originating from the simple, static slab are typically symmetric, a velocity field could also introduce asymmetric spectra depending on viewing angle, due to the fact that e.g. receding gas will have higher optical depth for blue photons, while it will be reduced for red photons.

Adding dust can render the effects of the anisotropies stronger in terms of the variations in flux. Since a higher optical depth leads to more scatterings, inducing more changes of direction, the pathlength increases when $\tau$ increases. The probability of being absorbed by dust is proportional to this pathlength, so dust becomes more effective in absorbing photons.

Although the simple toy model of a slab is of course much too simple to capture the complexity of real astrophysical conditions, it highlights how anisotropies can influence observed Lyman- $\alpha$ properties. Analogous to the optically thin and thick cases of the simple slab discussed above, we can identify the regimes of the intergalactic medium (IGM) with low densities $\left(<10^{-6} \mathrm{~cm}^{-3}\right.$ for hydrogen, dust content negligible) and the ISM where neutral hydrogen densities and dust content can be very high (e.g. $1 \mathrm{~cm}^{-3}$ for hydrogen).

Going back to the toy model proposed above, we see that in the optically thin case, it represents a very sketchy model of a filament with an embedded isotropically emitting galaxy. For large-scale surveys like HETDEX, this toy model implies that when we observe along the long axis of a filament, we might miss sources in the filament because photons are efficiently scattered out of our line of sight, while we do observe fractionally more sources residing in filaments that we observe along the short axis. In our toy model, this effect is very strong. Yet, our model is far from realistic. In particular, our configuration is static and has a very simplified geometry. In the work done by Zheng et al. 2011; Zheng et al. 2010 and Behrens \& Niemeyer 2013, cosmological simulations were used to estimate the effect on the measured matter distribution in a more realistic and consistent way. Their findings will be presented in the next section.

With respect to radiative transfer in the ISM, the optically thick (and dusty) slab model is only a very rough illustration, since we expect the geometry of LAEs to be either approximately spherical or disk-like, possibly with a very clumpy morphology. This will be investigated in detail in chapter 3. Assuming that emitters have a 
disk-like structure and - analogous to the slab model - a higher flux towards face-on directions, one would expect the transport of Lyman- $\alpha$ radiation in the ISM to influence large-scale surveys only as a source of noise, since one might assume that the orientations of the disk are distributed randomly and independent of the largescale environment. However, under certain plausible conditions, the anisotropy of the emitters themselves could influence the measurements of the large-scale structure, namely if there is a correlation between the large-scale structure and the orientation of galactic disks. This will be discussed in the second section of this chapter on the basis of the work by Hirata 2009.

\subsection{Contamination by Attenuation in the Large-Scale Structure}

In their work, Zheng et al. 2010 and Zheng et al. 2011 analyze the radiative transfer of Lyman- $\alpha$ photons within a cosmological simulation with a box size of $100 \mathrm{Mpc} / \mathrm{h}$ at a redshift of $z=5.7$. Apart from the Lyman- $\alpha$ physics as summarized in section 1.3 , they also include the Hubble flow and apply periodic boundary conditions (see section 1.3.3), but ignore dust. For each halo in their simulation volume, they define an intrinsic Lyman- $\alpha$ luminosity $L_{\text {intrinsic }}$ derived from the halo mass $M_{h}$ :

$$
L_{\text {intrinsic }}=10^{42} \frac{0.68 M_{h}}{10^{10} M_{\odot}} \operatorname{erg} / \mathrm{s}
$$

Here, $M_{\odot}$ is the mass of the sun. This relation is a result of combining the relation between intrinsic luminosity and star formation rate from Furlanetto et al. 2005 with a relation between star formation rate and halo mass found in the used simulation (see Trac \& Cen 2007). They launch a number of tracer photons from the center of each halo with mass above $5 \times 10^{9} \mathrm{M}_{\odot}$. Although the ISM not resolved in the simulation (resolution $\sim 32 \mathrm{kpc}$ ) and the achieved densities are correspondingly low, the anisotropic and inhomogeneous IGM surrounding the emitters can affect the observed emitter statistics, as has been illustrated in the introduction of this chapter. Nevertheless, the halo cores where the photons are launched are optically thick, which makes it necessary to follow their escape with the full radiative transfer simulation.

To quantify the influence of the large-scale environment on the Lyman- $\alpha$ properties, 
Zheng et al. 2010 calculate the correlations of the large-scale density contrast $^{1} \delta$, its derivative with respect to the line of $\operatorname{sight}^{2} \frac{\partial \delta}{\partial z}$, the velocity component along the line of sight $v_{z}$ and the velocity gradient along the line of sight $\frac{\partial v_{z}}{\partial z}$ with the observed fraction of Lyman- $\alpha$ emission, $\varepsilon=\log \left(L_{\text {apparent }} / L_{\text {intrinsic }}\right)$. These quantities are evaluated from the simulations by calculating them from a smoothed dark matter density field (smoothed on scales of $4 \mathrm{Mpc} / \mathrm{h}$ ) in the linear regime, i.e. using the linearized continuity equation to connect the overdensity or density contrast $\delta$ and velocity field $\vec{v}$ :

$$
\dot{\delta}+\frac{1}{a} \nabla \cdot \vec{v}=0
$$

$a$ is the scale factor. In the linear regime, different Fourier modes of the density field evolve independently and grow linearily. Therefore, the time derivative of the density field $\dot{\delta}$ can be rewritten on linear scales as $f H(a)$, where $f$ is the growth factor. Using this, we can calculate the desired quantities in terms of the Fourier-transform of the density field, $\delta_{\vec{k}}$ :

$$
\begin{aligned}
\vec{v} & =f H a \sum_{\vec{k}} \frac{i k_{z}}{k^{2}} \delta_{\vec{k}} \exp (i \vec{k} \vec{r}) \\
\frac{\partial v_{z}}{\partial z} & =-f H a \sum_{\vec{k}} \frac{k_{z}^{2}}{k^{2}} \delta_{\vec{k}} \exp (i \vec{k} \vec{r}) \\
\frac{\partial \delta}{\partial z} & =\sum_{\vec{k}} i k_{z} \delta_{\vec{k}} \exp (i \vec{k} \vec{r})
\end{aligned}
$$

This means that not the (non-linear) density/velocity field present in the simulation is used, but the linear approximation. This is done to remove the influence of the dense halo cores, since Zheng et al. 2010 are interested in the effects of the large scale structure. The results are shown in figure 2.3.

Intuitively, one would expect the density contrast $\delta$ near the location of an emitter to be correlated with a low observed fraction, since the probability for a photon escaping from that emitter to be scattered out of the line of sight is higher. This is indeed the case, Zheng et al. 2010 find an increase in observed fraction by a factor of

1 as usual, the overdensity or density contrast $\delta$ is defined as the local density over the mean density, $\delta=\rho / \bar{\rho}$

2 In the following, we assume that the direction of observation is along the $z$-axis. 

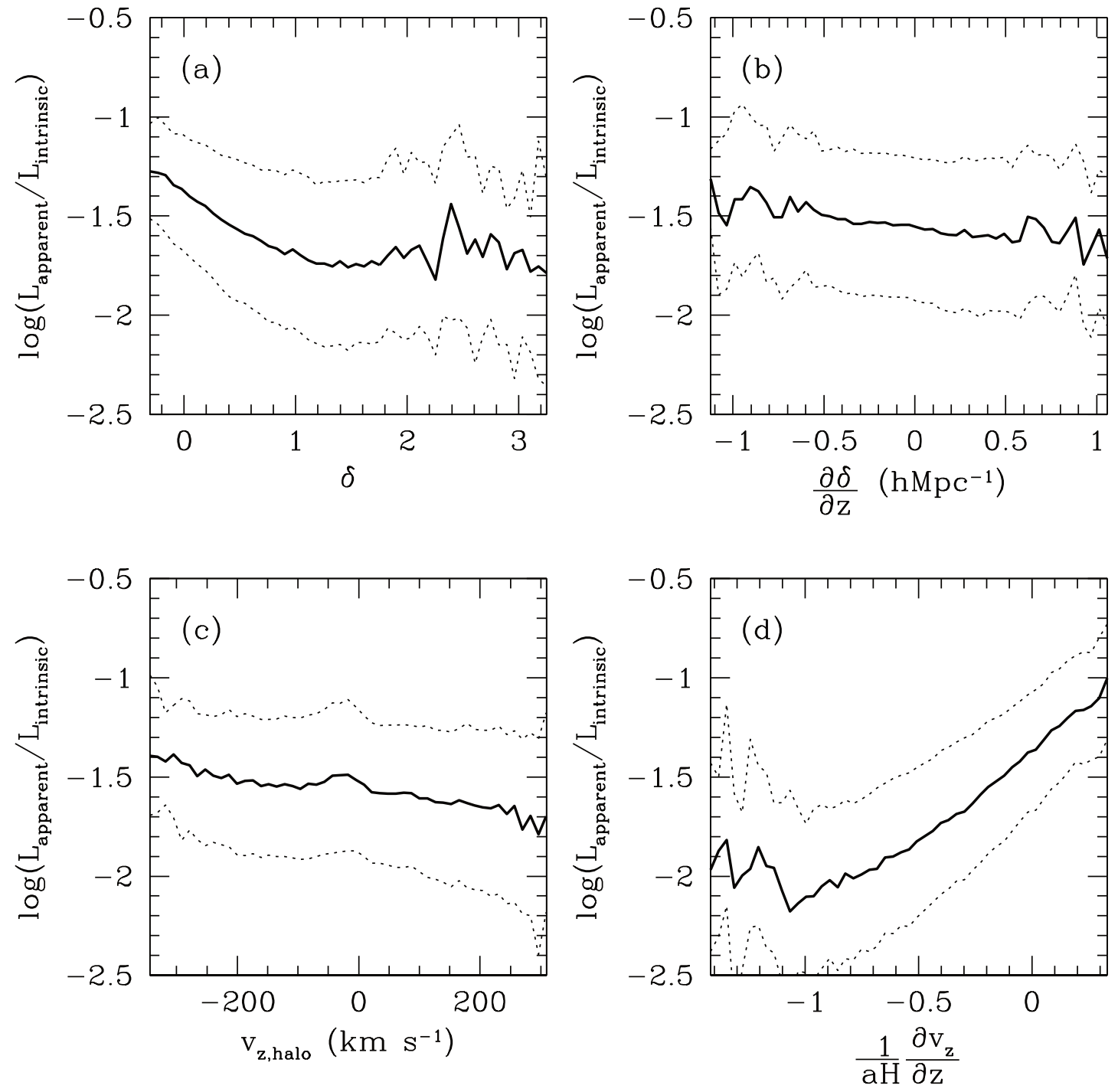

Figure 2.3: Correlations of large-scale environmental density (top left), line-of-sight density gradient (top right), line-of-sight velocity (bottom left), and line-of-sight velocity gradient (bottom right) with observed Lyman- $\alpha$ fraction as reported in Zheng et al. 2010. (c) AAS Reprinted with permission (Figure 21 in Zheng et al. 2010) 
$\sim 5$ at low density contrasts (see figure 2.3, upper left panel). However, they find the line-of-sight velocity gradient to have much larger impact on the observed fraction: The velocity gradient boosts the observed fraction by an order of magnitude. Zheng et al. 2010 explain this by noting that velocity gradients change the local Hubble rate. A positive velocity gradient along the line of sight increases the local Hubble rate, which in turn shifts photons away from the line center, reducing the optical depth photons have to penetrate to reach the observer.

Zheng et al. 2011 analyze these results in terms of the effects on the observed large-scale density structure. To do this, they construct mock observations, using an observation threshold on the apparent luminosity of their emitters. For comparison, they construct a halo catalogue with the same number density, and a 'shuffled' LAE catalogue with the apparent luminosity randomly shuffled among all emitters to remove any correlations between environment and LAE properties. Finally, they calculate the 2-point correlation function (2PCF) as a function of parallel separation and perpendicular separation $\left(r_{p}\right.$ and $\pi$ ) from these data sets. The $2 \mathrm{PCF}$ is defined to be the excess probability to find a source at distance $\left(r_{p}, \pi\right)$ from a source compared to a sample of objects with a random, flat distribution in space. We show their plot in figure 2.4. In the left center panel, the unbiased $2 \mathrm{PCF}$ of the halos is shown. As expected, the $2 \mathrm{PCF}$ has approximately spherically shaped contours, indicating that the probability to find a galaxy with a line-of-sight separation $r_{p, s}$ from another galaxy is about the same as the probability to find a galaxy at a perpendicular separation $\pi_{s}=r_{p, s}$. On the other hand, the $2 \mathrm{PCF}$ of the LAE sample (top left panel) shows an elongation along the perpendicular direction, the contours are severely deformed by the effects of the radiative transport. As the left bottom panel shows, this deformation does not occur if one randomly shuffles the LAE properties among the emitters. The deformation therefore originates from the correlations of the environment of emitters and their Lyman- $\alpha$ properties.

As Zheng et al. 2011 suggest, this elongation pattern is due to the strong correlation between line-of-sight velocity gradient and observed fraction. The mechanism for this is illustrated in figure 2.5, left panel. In gray, the density contours are shown for a density fluctuation illustrated as a plane wave. Dots represent emitters, the ellipsoids illustrate the angular distribution of relative transmitted flux. For better understanding, we plot an illustration of density, line-of-sight velocity, and lineof-sight velocity gradient in figure 2.6. The velocity gradient follows the density gradient, and because of the correlation between velocity gradient and observed 
fraction, one expects emitter in the denser regions in the left panel of figure 2.5 to transmit photons preferentially perpendicular to the line of sight, since the velocity gradient within the overdensity is negative along the line of sight (see figure 2.6, label 'c)'), while it is close to zero perpendicular to it. This means that emitters in this region have a lower probability of being detected. Emitters in underdense regions in turn transmit Lyman- $\alpha$ radiation preferentially in the directions parallel to the observer, since the line-of-sight velocity gradient is positive in these directions (see figure 2.6, label 'a)'), so emitters in these regions have higher probability of being detected. Emitters with zero line-of-sight velocity gradient (label 'b' in figure 2.6) have no preferred escape direction. If the density mode is perpendicular to the line of sight (right panel of figure 2.5), the situation is reversed ${ }^{1}$. Now overdense regions seem more overdense to the observer because of the higher probability for emitters in the overdense region to be detected, while the underdense regions appear even less dense.

However, Behrens \& Niemeyer 2013 (see section 4.1) investigate these effects on the basis of a cosmological simulation with a smaller box size $(50 \mathrm{Mpc} / \mathrm{h})$, but at higher resolution (1 kpc vs. $32 \mathrm{kpc}$ physical) and with full treatment of the hydrodynamics instead of a hybrid-scheme ${ }^{2}$ as used by Zheng et al. 2010, at redshifts of $z=2-4$. We calculate correlations of the observed fraction of Lyman- $\alpha$ emission with the large-scale structure in the same way as Zheng et al. 2010, but smooth the density field on a larger scale of $10 / 12 / 15 \mathrm{Mpc} / \mathrm{h}$ at $z=2 / 3 / 4$ to be in the linear regime. As can be seen in figure 5 in section 4.1 (note that the $y$-axis shows observed fraction relative to the mean), we obtain similar correlations for the local overdensity $\delta$ (upper left panel): Higher densities suppress the observed fraction by $\sim 30 \%$. On the other hand, we do not find correlations as strong as Zheng et al. 2010, in particular for the line-of-sight velocity gradient. While the observed fraction changes by an order of magnitude in their simulation, we find a change of about $20 \%$. With respect to the other analyzed correlations, we refer the reader to section 4.1 .

We also calculated the $2 \mathrm{PCF}$ arising from the radiative transfer effects. It is shown

1 We note that for this statement, Zheng et al. 2011 employ a new assumption: They assume that the relative flux in a direction does not only depend on the velocity gradient in this direction, but also on the gradient in all other directions. Otherwise, the right panel of figure 2.5 would show equal flux to the observer independent of the local density, since the line of sight velocity gradient is zero for all sources.

2 This means that the dark matter evolution was simulated using a high-resolution N-body simulation, while the gas was prescribed onto the dark matter distribution as a function of the local dark matter distribution, see Trac \& Cen 2007. 

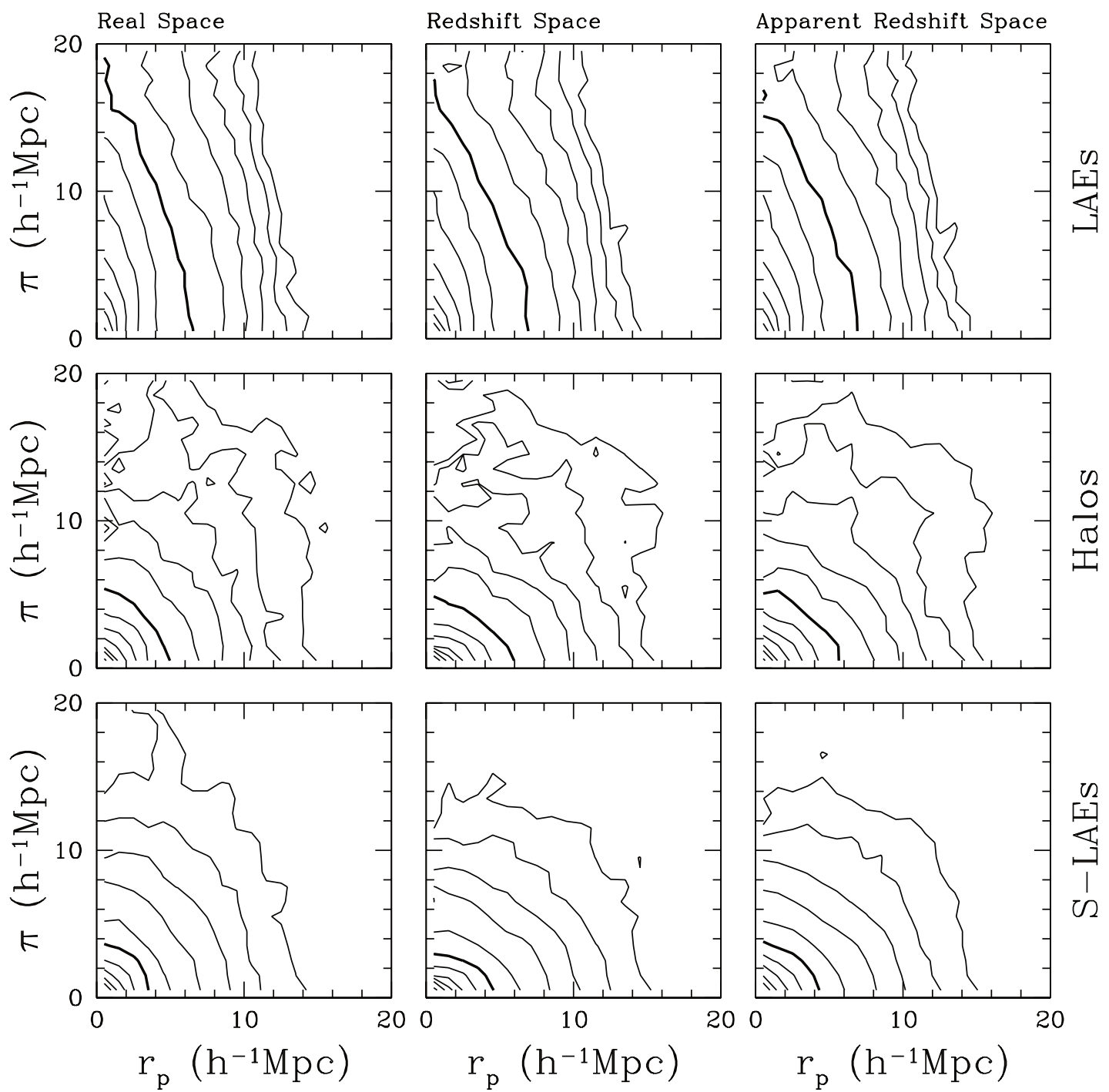

Figure 2.4: 2PCFs from Zheng et al. 2011 in real space (left column), redshift space (center column), and apparent redshift (right column) for samples of LAEs (first row), halos (second row), and a sample of LAEs for which the Lyman- $\alpha$ properties have been randomly shuffled (last row). The 2PCFs are shown as a function of parallel separation $\left(r_{p}\right)$ and orthogonal separation $(\pi)$. (c) AAS Reprinted with permission (Figure 7 in Zheng et al. 2011) 

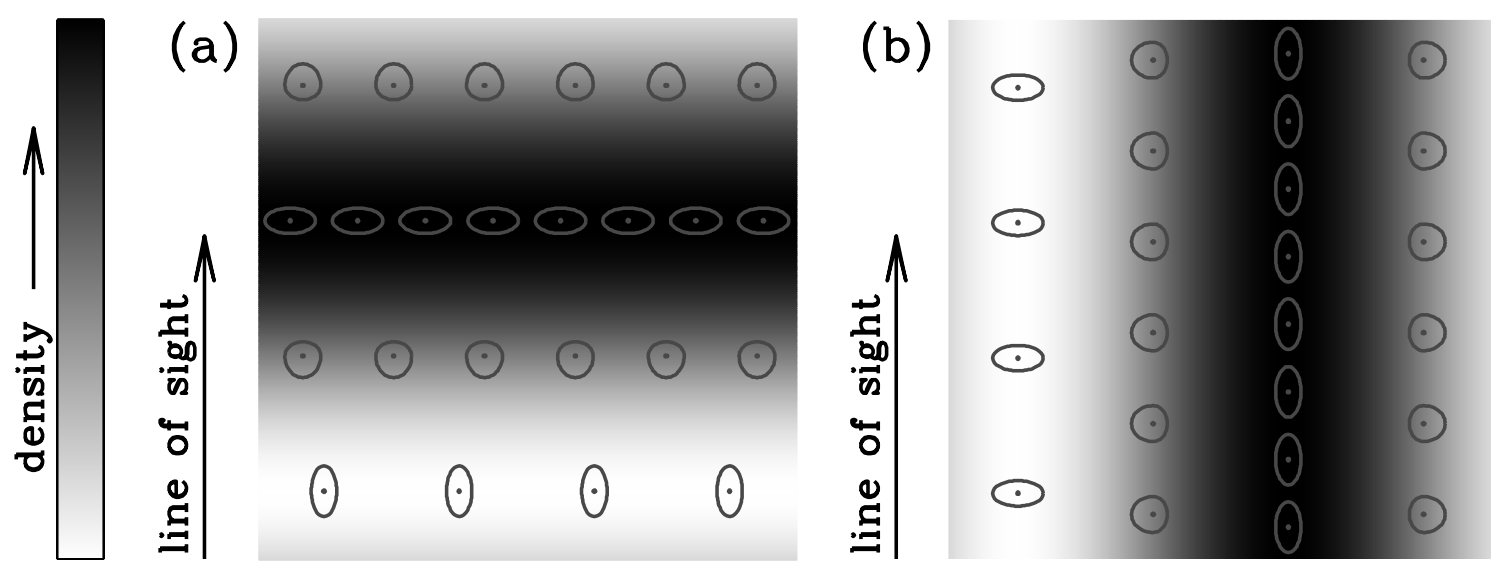

Figure 2.5: The mechanism causing the observed deformation of the $2 \mathrm{PCF}$ as depicted by Zheng et al. 2011. Dots indicate emitters, the ellipsoids around it the relative flux escaping in a given direction. The background shading illustrates density modes parallel and perpendicular to the observer. Lyman- $\alpha$ photons from galaxies in an overdense region escape predominantly in the direction of the filament, while those from emitters in underdense regions escape along the line of sight. This leads to suppression of modes parallel to the observer and enhancement of modes perpendicular to the observer. (c) AAS Reprinted with permission (Figure 9 in Zheng et al. 2011)

in figure 12 in section 4.1. The sample is again a threshold sample with approximately the same number density as in Zheng et al. 2011. We do not find evidence for a deformation of the $2 \mathrm{PCF}$ for the LAEs (right panel), the $2 \mathrm{PCF}$ looks very similar to the $2 \mathrm{PCF}$ of the shuffled LAE sample (center) and the $2 \mathrm{PCF}$ of the halos (left panel). To analyze this further, we used a multipole expansion of the $2 \mathrm{PCF}$. The deformation of the $2 \mathrm{PCF}$ should be clearly visible in the quadrupole, which is plotted in figure 14, section 4.1, where the black/red/green line shows the quadrupole for the halo/LAE/shuffled LAE sample. We do not find a significant contribution to the quadrupole compared to the multipole expansion of the halo/shuffled LAE sample.

The difference between our results and the results of Zheng et al. 2011; Zheng et al. 2010 can be partly explained by the fact that the redshifts at which the simulations were done are different. The higher mean density $(\sim 100$ times higher, comparing $z=4$ analyzed by Behrens \& Niemeyer 2013 and $z=5.7$ analyzed by Zheng et al. 2010) at the higher redshift of $z=5.7$ increases the impact of the environment on the Lyman- $\alpha$ transport locally. Additionally, the hybrid scheme employed by Zheng et al. 2010 could artificially boost the correlation of the gas distribution and the dark matter distribution compared to a full hydrodynamical treatment of the gas. Finally, it is possible that our box volume is too small to detect the signal, since we 


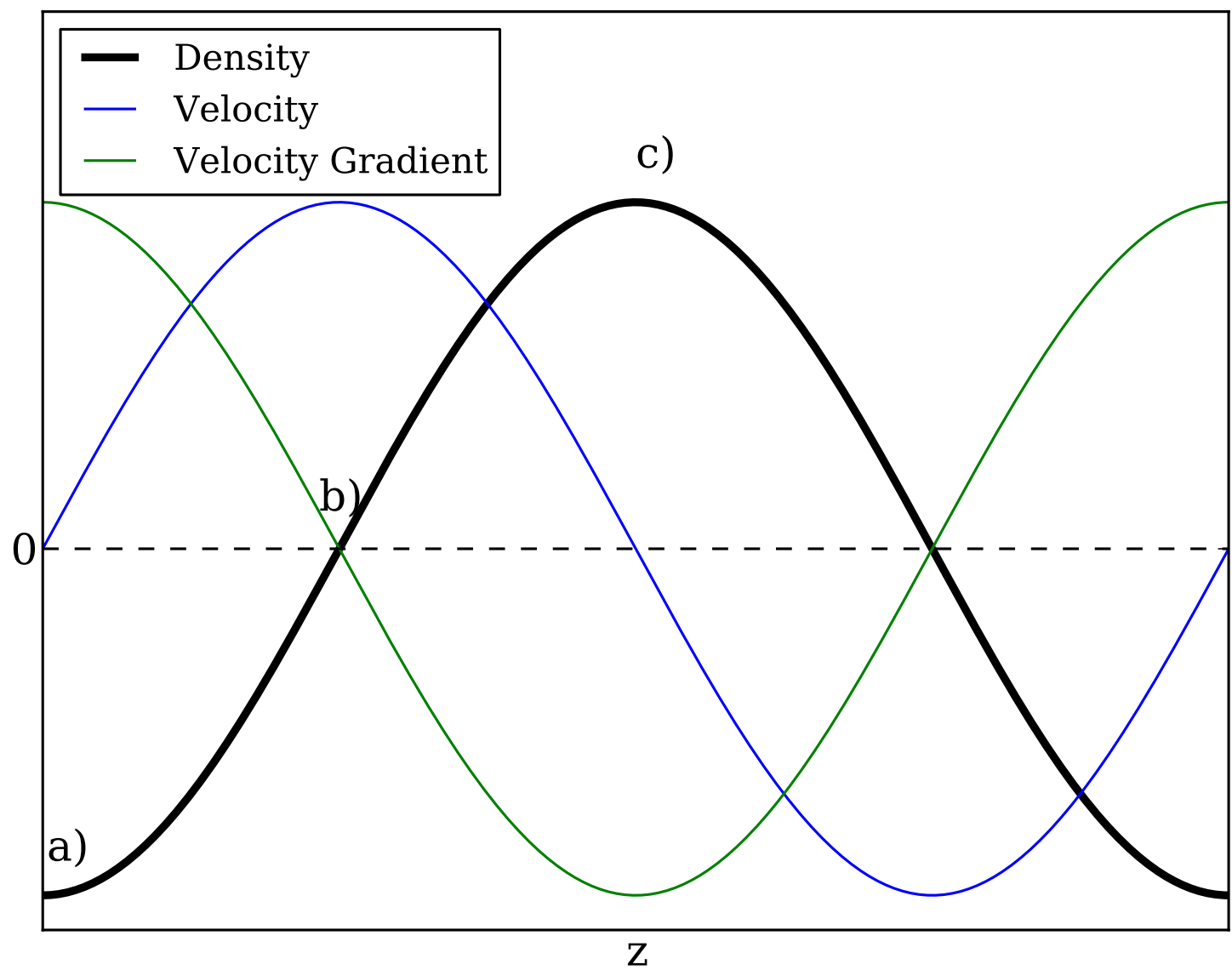

Figure 2.6: Illustration of density, line of sight velocity, and line of sight velocity gradient for a plane-wave density perturbation.

only have $1 / 8$ of the volume of Zheng et al. 2010 .

In an analytical framework, Wyithe \& Dijkstra 2011 show how the connection between the environment of the emitters and observed Lyman- $\alpha$ properties can be taken into account. They focus on the effects in the power spectrum and derive expressions for the influence of the local velocity gradient (parametrized by the quantity $\left.C_{v}\right)$, the local density $\left(C_{\rho}\right)$ and the local ionization $\left(C_{\Gamma}\right)$ in a first-order approximation. For the modified power spectrum $P_{L y \alpha}(k, \mu)$ as a function of the true matter power spectrum $P(k)$, they derive

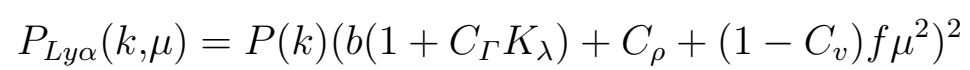

If no radiative transfer effects are present (all $C$-parameters equal to zero), this 
equation is reduced to the usual relation between the matter and galaxy power spectrum, with $b$ the galaxy bias (Kaiser 1984) and $f \mu^{2}$ the redshift distortion term (Kaiser 1987). $K_{\lambda}$ is a function of the mean free path $\lambda$ of ionizing photons, effectively parametrizing how the density of Lyman- $\alpha$ sources and the resulting fluctuations in ionization are related. We can already see from this equation that there is a problematic degeneracy between redshift distortions (last term in the parentheses), galaxy bias (first term) and the $C$-parameters introducing radiative transfer effects. This means that naively, if the radiative transfer effects are not negligible, large-scale surveys cannot distinguish between them and effects originating from the growth factor $f$ or the bias factor $b$.

To be more specific, the $C$-parameters are proportional to

$$
\begin{aligned}
C_{\rho} & \left.\propto \frac{\partial(\log (T))}{\partial(\log \rho)}\right|_{T_{0}, \rho_{0}} \\
C_{v} & \left.\propto \frac{\partial(\log (T))}{\partial\left(\log \left(d v_{z} / d r\right)\right)}\right|_{T_{0}, \Gamma_{0}} \\
C_{\Gamma} & \left.\propto \frac{\partial(\log (T))}{\partial(\log \Gamma)}\right|_{T_{0}, \Gamma_{0}}
\end{aligned}
$$

In these equations, $\rho$ is the hydrogen density, $d v_{z} / d r$ is the line-of-sight velocity gradient, $\Gamma$ is the local photoionization rate, and $T$ is the Lyman- $\alpha$ transmission. The $C$-parameters measure how strongly the transmission varies with the local density/velocity gradient/ionization parameter, and have to be evaluated from models for the radiative transfer. We discuss here the results from the simple analytic model that Wyithe \& Dijkstra 2011 present (their section 3.2). From equation 2.7, we can see how the different $C$-parameters affect the power spectrum. This is also shown in figure 2.7 for the spherically averaged power spectrum. The grey line shows the case where $C_{\Gamma}=C_{\rho}=C_{v}=0$, whereas the other lines show the power spectrum in the case where $C_{\rho}$ (dashed), $C_{\rho}$ and $C_{\Gamma}$ (solid), or $C_{\rho}, C_{\Gamma}, C_{v}$ (long-dashed) are $\neq 0$. As we can see, the contribution from $C_{\rho}$ simply reduces the amplitude of the power spectrum. This is consistent with the finding of Zheng et al. 2010 that dense regions tend to suppress the observed Lyman- $\alpha$ fraction. In the simple model considered, having contributions from $C_{v}$ alone has a similar effect. Adding the contribution from $C_{\Gamma}$ increases power on large scales, since overdense regions tend to have higher ionization parameters, making it easier for photons to escape in the large-scale environment of these regions. Including all contributions results 
in a power spectrum of similar shape, but with slightly lower amplitude due to the inclusion of $C_{v}$.

Wyithe \& Dijkstra 2011 also investigate the findings of Zheng et al. 2010 and Zheng et al. 2011 in terms of their more general analytic framework. In their terminology, the observed correlation of large-scale velocity gradient and observed fraction translates to a high value of $C_{v}>1$, corresponding to the change of the transmission with respect to a velocity gradient being large. The power spectrum as a function of parallel and transverse separation of such a case is shown in figure 2.8 (right panel). The interpretation of this plot is similar to the $2 \mathrm{PCF}$, but the units on both axes are now $h / \mathrm{Mpc}$ instead of Mpc. For comparison, the left panel in the figure shows the power spectrum with all $C$-parameters equal to zero. As we can see, the model qualitatively reproduces the deformation observed by Zheng et al. 2011. With respect to Behrens \& Niemeyer 2013, their model indicates that, given the $C_{v}$ implied by the derivative of the relative observed fraction $\varepsilon$ with respect to the line-of-sight gradient, a small deformation would have been expected $\left(C_{v}=0.5\right.$, private communication with M. Dijkstra). In this respect, the current results remain inconclusive with respect to the question if there is a significant deformation of the measured $2 \mathrm{PCF}$, but it seems plausible that the possible effect is much smaller at redshifts relevant for large-scale surveys to date than found by Zheng et al. 2011 at a correspondingly higher redshift. In particular, Behrens \& Niemeyer 2013 also find that the correlations strongly decline at lower redshift, rendering radiative transfer effects ineffective (see 4.1, figure 7).

Additionally, it is also worth noting that Greig et al. 2013 point out that the problem can be overcome by breaking the degeneracy of radiative transfer effects and usual clustering effects by using information from the bispectrum (which is a higher-order measure of the statistical properties of the sample), but to do this precisely, information on the strength of the effect is beneficial, apart from the fact that the bispectrum is naturally more difficult to measure than the power spectrum or $2 \mathrm{PCF}$. 
$F=0.9, \quad z=3.0, \quad \tau_{0}=1, \quad \lambda=300, \quad \beta=2$

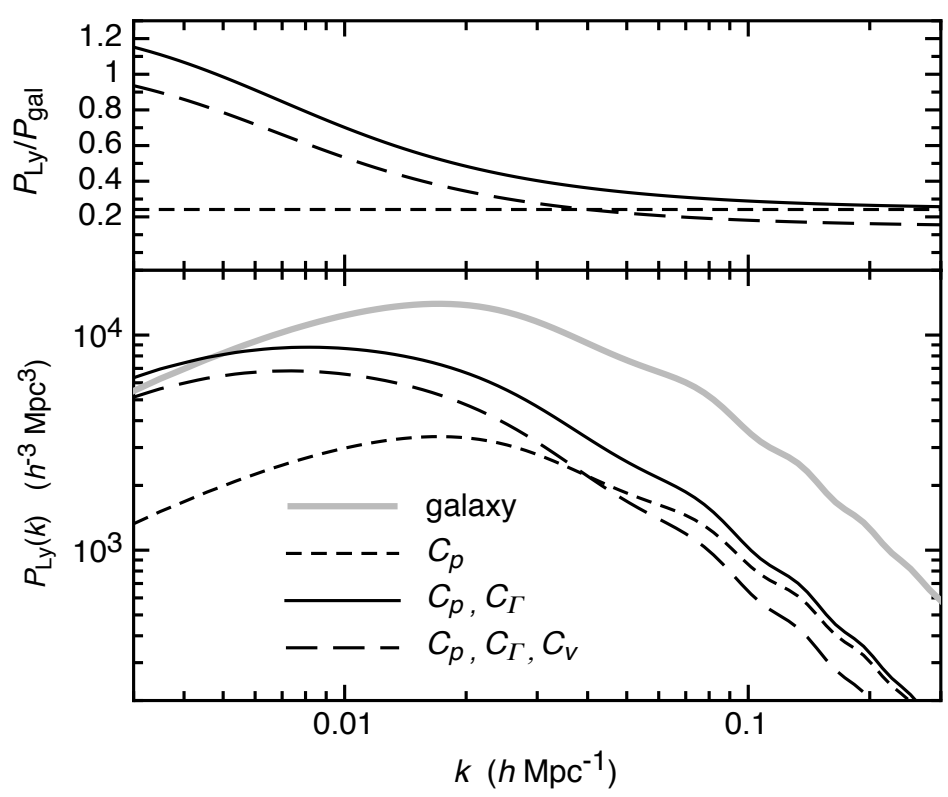

Figure 2.7: Shown are the different contributions to the power spectrum from the radiative transfer effects, parametrized by $C_{\rho}, C_{v}$, and $C_{\Gamma}$. The grey line shows the case where all $C$-parameters are zero. The dashed line corresponds to $C_{\rho} \neq 0$, the solid line to $C_{\rho}, C_{\Gamma} \neq 0$, the long-dashed line corresponds to $C_{\rho}, C_{\Gamma}, C_{v} \neq 0$. Reprinted with permission (Figure 2 in Wyithe \& Dijkstra 2011)
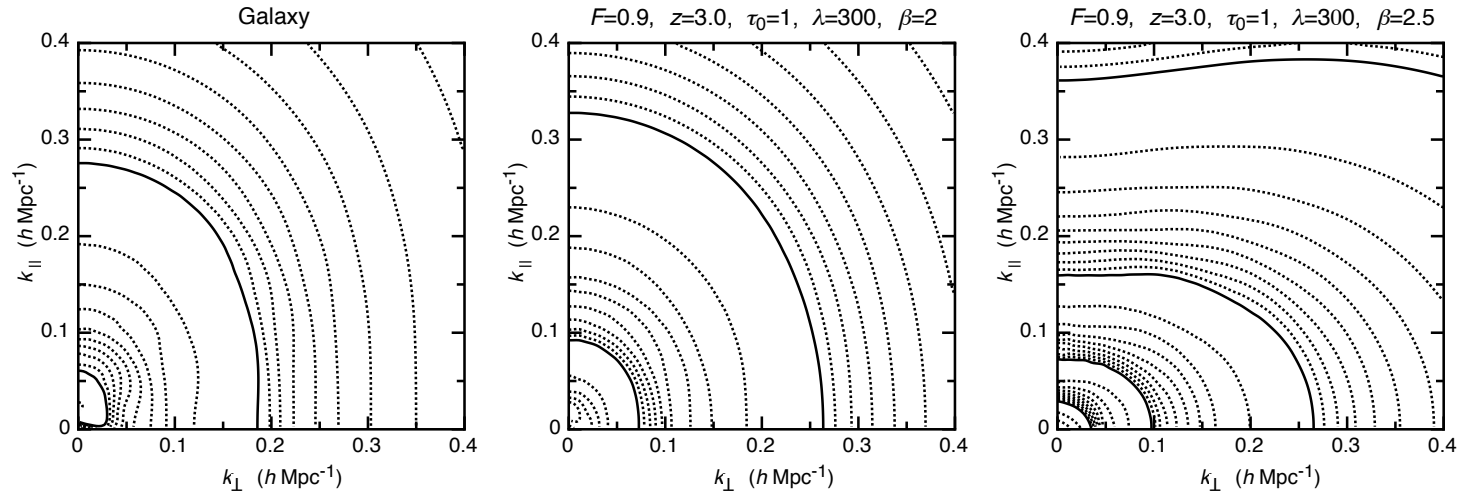

Figure 2.8: Shown is the power spectrum as a function of transverse and parallel separation $\left(k_{\perp}, k_{\|}\right)$for the analytical model without any radiative transfer effects (left panel) and for two model including the effects. While the middle panel has $C_{v}<1$, the right panel has $C_{v}>1$, leading to the deformation observed in the simulations by Zheng et al. 2011. Note that the axis here are in $k$-space. Reprinted with permission (Figure 3 in Wyithe \& Dijkstra 2011) 


\subsection{Contamination from Anisotropic Escape and Tidal Alignment}

The second effect that we will discuss in detail here can affect clustering statistics by connecting the properties of large scales probed by surveys with the local conditions on scales of the ISM of an emitting galaxy, namely by the statistical alignment of the orientation of galaxies within the large-scale environment due to tidal interaction. Such a mechanism was proposed and studied by Hirata 2009. Additionally to tidal alignment, one needs a varying detectability of emitters with inclination for this mechanism to become effective.

For illustration, if we assume that a population of disky galaxies has less line flux escaping edge-on than face-on, and these galaxies have spins that are aligned perpendicular to the large-scale density modes, we find that observations will miss more sources in modes parallel to our line of sight depending on the flux limit of the survey. This would ultimatively result in a systematic error of the measured correlation functions. An illustration of the mechanism outlined above is shown in figure 2.9.

Hirata 2009 discuss this effect using a linear model for the intrinsic alignment of
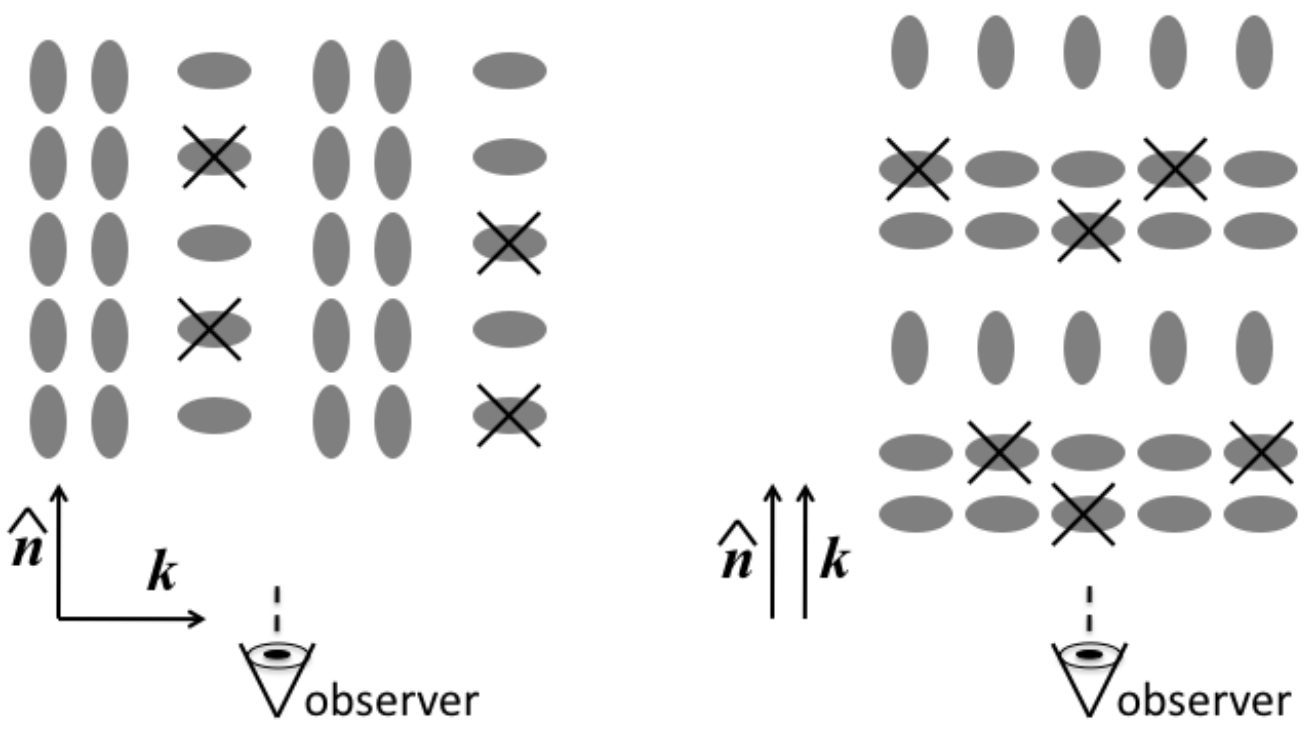

Figure 2.9: Illustration of the Hirata-effect. $\vec{k}$ denotes the orientation of the considered mode of the density field, $\vec{n}$ the line of sight. If galaxies, depicted as ellipticals here, are aligned within the large-scale density field, and the observability depends on the orientation towards the observer, emitter statistics can be systematically affected because the probability of missing a source is higher in certain environments. Reprinted with permission (Figure 1 in Hirata 2009) 
galaxies in the large-scale tidal field. We will briefly summarize this work here.

We call $P_{A}(\mathbf{Q} \mid \vec{x})$ the probability distribution of a galaxy located at $\vec{x}$ to have orientation $^{1} \mathrm{Q}$. Without intrinsic alignment, we find that $P_{A}$ is independent of $\vec{x}$ (and constant), but in the presence of tidal alignments, $P_{A}$ will depend on the large-scale density field at $\vec{x}$. We define a selection function

$$
P_{S} \propto 1+\Upsilon(\mathbf{Q} \vec{n} \mid \vec{x})
$$

that yields the probability for detecting a galaxy with orientation $\mathbf{Q}$ at position $\vec{x}$ given that the line of sight is $\vec{n}$. $\Upsilon$ introduces the anisotropy here; in the isotropic case, it would be zero since the detection probability of a galaxy would not depend on its orientation towards the observer. Connecting both the probability of a certain orientation and the detectability resulting from this orientation yields the quantity

$$
\varepsilon(\vec{n} \mid \vec{x})=\int_{S O(3)} P_{A}(\mathbf{Q} \mid \vec{x}) \Upsilon(\mathbf{Q} \vec{n} \mid \vec{x}) d^{3} \mathbf{Q}
$$

where we integrated over all orientations, i.e. the rotation group. The effect modifies the relation between the observed galaxy density $\delta_{g}$ and the true matter density $\delta_{m}$ in redshift space. Hirata 2009 find

$$
\delta_{G}(\vec{k})=\left(b+f \mu^{2}\right) \delta_{m}(\vec{k})+\varepsilon\left(\vec{e}_{3} \mid \vec{k}\right)
$$

where the frame of reference has been transformed to be along $\vec{e}_{3}$. The terms in parentheses represent the bias factor $b$ (Kaiser 1984) relating the galaxy density with the matter density, and the usual redshift distortion (Kaiser 1987) induced by the peculiar motions of the galaxies, $f \mu^{2}=f \overrightarrow{e_{3}} \vec{k}$.

The task now is to give an expression for $\varepsilon$. We skip the technical details of the calculation here and state the result relating the observed galaxy power spectrum to the true matter power spectrum $P_{m}$, as given by Hirata 2009:

$$
P_{g}(\vec{k})=\left(b-\frac{A}{3}+(f+A) \mu^{2}\right)^{2} P_{m}(\vec{k})
$$

The term $A$ is an expansion coefficient that incorporates the effects of the tidal

$1 \mathbf{Q}$ is considered a rotation matrix indicating how to transform an observer frame into a frame aligned with the axis of the galaxy. 
alignment and selection bias and is related to $\varepsilon$ :

$$
\varepsilon(\vec{n} \mid \vec{x})=A s_{i j}(\vec{x}) \vec{n}_{i} \vec{n}_{j}
$$

with $s_{i j}$ the dimensionless tidal field:

$$
s_{i j}=\left(\nabla_{i} \nabla_{j} \nabla^{-2}-\frac{1}{3} \delta_{i j}\right) \delta_{m}(\vec{x})
$$

Before stating $A$ for the particular case of disk galaxies, we can already see from equation 2.14 that the tidal alignment modifies the power spectrum in two ways: $A$ reduces the bias $b$, and it contributes to the redshift distortion term. As Hirata 2009 point out, the first contribution is degenerate with the galaxy bias $b$, while the latter is degenerate with the growth factor $f$. Of course, these degeneracies pose a problem for large-scale surveys, since the different effects cannot be disentangled by using the power spectrum/2PCF alone as described above for the Zheng effect.

To quantitatively examine the impact of the Hirata effect, one has to determine $A$. We summarize how this can be done for galaxies dominated by a thin disky component. One starts by noting that the number density $N\left(F_{i}, \vec{L}\right)$ of galaxies as a function of logarithmic intrinsic flux $F_{i}$ and orientation $\vec{L}$ can be written as

$$
N\left(F_{i}, \vec{L}\right)=F_{i}^{-\eta}\left(1+B s_{i j} \vec{L}_{i} \vec{L}_{j}\right)
$$

In this equation, $\eta$ is the slope of the flux function, i.e. the cumulative luminosity function $\bar{n}$ has the property

$$
\frac{\mathrm{d} \ln \bar{n}}{\mathrm{~d} \ln F_{\min }}=-\eta
$$

with $F_{\min }$ the flux limit of the survey. $B$ parametrizes the amplitude of the tidal alignment effect on the orientations.

Integrating this over all orientations to find the number density above a certain flux $F_{0}$ yields after some calculations:

$$
N\left(>F_{0}\right) \propto \int_{0}^{\pi} \Phi(i)^{\eta}\left(1+B s_{33} P_{2}(\cos i)\right) \sin (i) d i
$$

where $\cos i$ the inclination, $P_{2}$ is the Legendre polynomial, and $\Phi(i)=F / F_{i}$ encodes the inclination dependence of the flux. Comparing this with the definition of $\varepsilon$, one 
sees that the anisotropy of the selection function comes from $s_{33}$. Therefore, one can write

$$
\varepsilon(\vec{n} \mid \vec{x})=B s_{33} \frac{\int_{0}^{\pi} \Phi(i)^{\eta} P_{2}(\cos i) \sin (i) d i}{\int_{0}^{\pi} \Phi(i)^{\eta} \sin (i) d i}=-B s_{33} \psi
$$

and comparing with the definition of $A$ in equation 2.15 , we finally find that

$$
A=-\psi B
$$

Hirata 2009 proceeds to express $B$ in terms of the parameters used by Bernstein 2009 to quantify $B$ which we will skip here. They conclude that for a 'pessimistic case', where $\Phi(i) \propto \cos (i)^{0.4}$ and a parameter $B$ at the $2 \sigma$ upper limit according to Hirata et al. 2007, $|A|$ becomes $\sim 0.039$, leading to a contamination of about $6 \%$.

As we have seen, the critical physical ingredients for estimating the systematic errors due to the Hirata effect in a large-scale survey are

1. A model for the tidal alignment of galaxies itself, here realized by a linear model and parametrized via $B$

2. A model for the dependence of the flux on the orientation of the galaxy towards the observer, labeled $\Phi(i)$ in the case of disky galaxies

In the special case of LAEs, the flux dependency $\Phi(i)$ can differ dramatically from the model used by Hirata 2009. As has been argued in the beginning of this chapter, the reason is the resonant scattering of Lyman- $\alpha$ photons in an optically thick hydrogen distribution. It is therefore very important to quantify this dependency, e.g. by numerical simulations, which requires detailed modeling of LAEs. Indeed, realistic models of individual disk galaxies, as presented by Verhamme et al. 2012 and Behrens \& Braun 2014 indicate that the inclination dependency of flux for Lyman- $\alpha$ radiation is very complicated and can differ significantly from the treatment in Hirata 2009. Interestingly, even for the radiative transfer in a cosmological volume with low resolution (1 kpc), Behrens \& Niemeyer 2013 find a significant variation of flux with respect to the inclination (see figure 15 in section 4.1). We will discuss the current state of the art of modeling LAEs in the next chapter.

It is worth noting that also the other ingredient of the Hirata model, namely the alignment of the galaxies' orientation within the large-scale structure, is subject to considerable uncertainty. For luminous red galaxies (LRGs) there is some ob- 
servational evidence that there is such a correlation (e.g. Faltenbacher et al. 2009; Hirata et al. 2007; Mandelbaum et al. 2006). A number of publications have tried to investigate these issues in simulations. The problem can be split into two parts, namely

- the alignment of dark matter halos with their large-scale environment and

- the alignment of galaxies with their dark matter halos.

Several authors found evidence for a non-vanishing alignment of dark matter halos with the large-scale structure (e.g. Aragon-Calvo et al. 2007; Codis et al. 2012; Libeskind et al. 2013). These authors find an alignment of halo spin parallel to the direction of the filament for low-mass halos, and an orientation perpendicular to the filament above a certain mass threshold (which may also depend on other quantities, see Libeskind et al. 2013). The reported spin-flip is explained by the fact that low-mass halos grow by accretion, while high-mass halos grow from (major) merger events, flipping their spin. However, Trowland et al. 2013 find that at high redshifts, most of the halos are orientated perpendicularly, and only the low mass halos exhibit a flip spin at low redshift.

The alignment of galaxy spins with the spin of the host halo is also a subject of ongoing research, especially because it is still computationally challenging to run simulations for a large volume of well-resolved, realistic galaxies. There is agreement in that a correlation between both quantities exists, but the exact amplitude and shape is a matter of debate (e.g. Codis et al. 2014; Dubois et al. 2014; Hahn et al. 2010). In particular, there is evidence for a statistically significant misalignment of galaxy spins with the halo spin. The (mis)alignment is influenced by resolution effects, i.e. the correlation between halo and galaxy spin changes with resolution (see Hahn et al. 2010). 


\section{CHAPTER 3}

\section{State of the Art: LAE Modeling}

In this chapter, we briefly review the literature on the modeling of LAEs for simulations of the radiative transport. We start with simplified, isotropic models and extend our discussion to anisotropic, simplified models. These two groups of models have in common that they are designed to be toy models with simplified geometries that catch important physical mechanisms of the radiative transfer of Lyman- $\alpha$ photons, but not the complexity of a realistic emitter. In the third section, we turn to more complicated models, derived from self-consistent simulations of individual galaxies or a number of galaxies embedded in a cosmological volume. We leave out the subject of Lyman- $\alpha$ modeling by semianalytic models (e.g. Dayal et al. 2011).

\subsection{Isotropic Simplified Models}

The simplest models of LAEs that have been investigated in the literature are models of a homogeneous sphere (or a slab, but we will neglect those here), possibly with an isotropic radial velocity field. Lyman- $\alpha$ photons are emitted at the center of the sphere, and at line center in frequency space. The main reason for considering these models is the existence of analytic expressions for the spectra emerging from them, at least in the static case (Dijkstra et al. 2006; Harrington 1973; Neufeld 1990). Since the numerical calculation of the radiative transport only became feasible in the last 10-20 years, these models were the only ones that could be studied. In the recent literature, these models are mostly used for testing the employed radiative transfer codes. Nevertheless, they give important first insights into the origin of spectral features of LAEs. Additionally, this class of models has also been studied as a simple model of a forming galaxy surrounded by approximately spherically distributed collapsing gas (e.g. Dijkstra et al. 2006), and as a model for neutral gas 
clouds illuminated by an external source (e.g. Verhamme et al. 2006).

We show some results for the static, spherical model in figure 3.1. We find a symmetric, double-peaked spectrum for these models, while there is effectively no flux at the line center with frequency $x=0$ (at least in the optically thick regime) where the photons were inserted. The reason is that in the line center, the optical depth is too high for photons to escape. Photons with a frequency close to the line center do not travel large spatial distances, since they are scattered many times. Only if they are scattered out of the line center into the 'wings' of the Voigt profile (see 1.3), they travel some distance before they are scattered again. Scatterings of photons far away from the line center shift them back into the line center on average. Typically, photons escape after a few of those excursions to the wings (Adams 1972). Since the gas is static, the optical depth for a photon at frequency $+x$ is the same as for a photon at $-x$, so the resulting spectrum must be symmetric. The two peaks reflect the typical frequency at which a photon can escape in a single flight without being scattered again. Analytically, one finds that the position of the peaks is related to the optical depth in the line center $\tau_{0}$ and the Voigt parameter $a$ (Dijkstra et al. 2006):

$$
x_{\text {peak }}= \pm 0.92\left(a \tau_{0}\right)^{1 / 3}
$$

If we add an isotropic radial velocity field that is zero in the center of the sphere and grows linearily further out with a maximum of $v_{\max }$ at the boundary of the sphere $^{1} R$ (i.e. Zheng \& Miralda-Escude 2002),

$$
v_{r}=\frac{r}{R} v_{\max }
$$

we find solutions as depicted in figure 3.2, here for three different values of $v_{\max }$. Focusing on the $20 \mathrm{~km} / \mathrm{s}$ case (blue line), we see that the spectrum becomes asymmetric. This is intuitive, since for photons with negative frequencies $-x$ (which we can also call 'red') moving outwards, the optical depth is reduced due to the bulk flow - in the rest frame of the outflowing gas, these photons appear even redder, lowering the effective cross section. On the other hand, photons in the blue part of the spectrum are closer to the line center in the rest-frame of gas, reducing their

1 We set $v_{r}=0$ for $r>R$ 


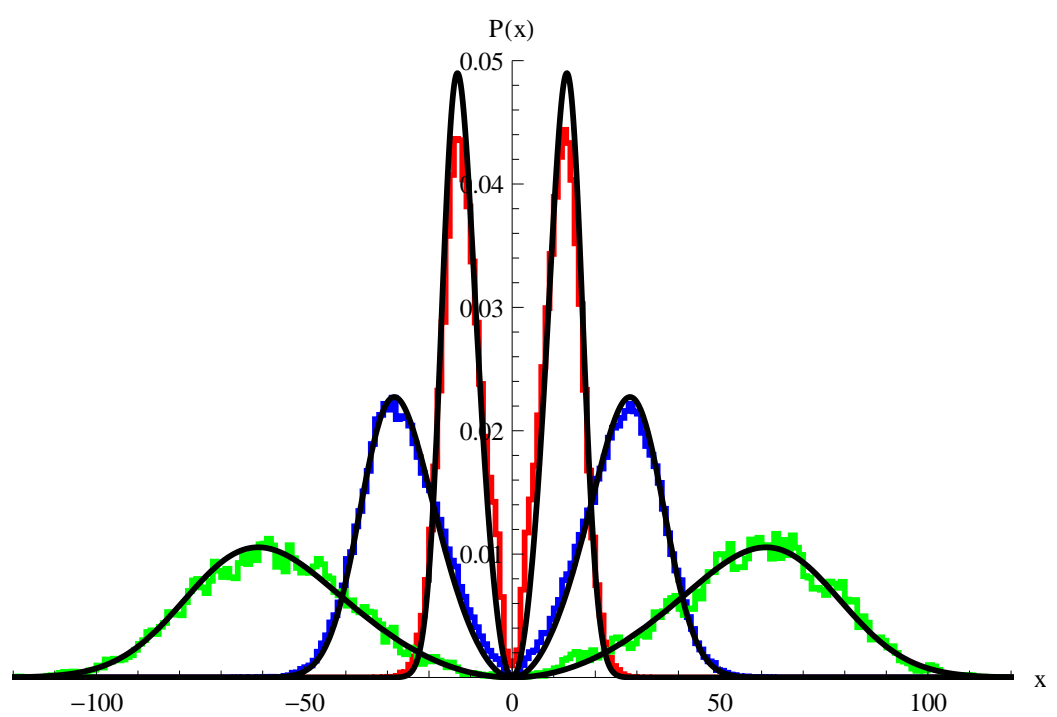

Figure 3.1: Spectra of a homogeneous, isothermal $\left(T=2 \times 10^{4} \mathrm{~K}\right)$ sphere with an optical depth of $\tau_{0}=10^{5}$ (green), $10^{6}$ (blue), and $10^{7}$ (red). Overplotted in black are the corresponding analytical solutions as obtained by Dijkstra et al. 2006. Taken from Behrens 2011.

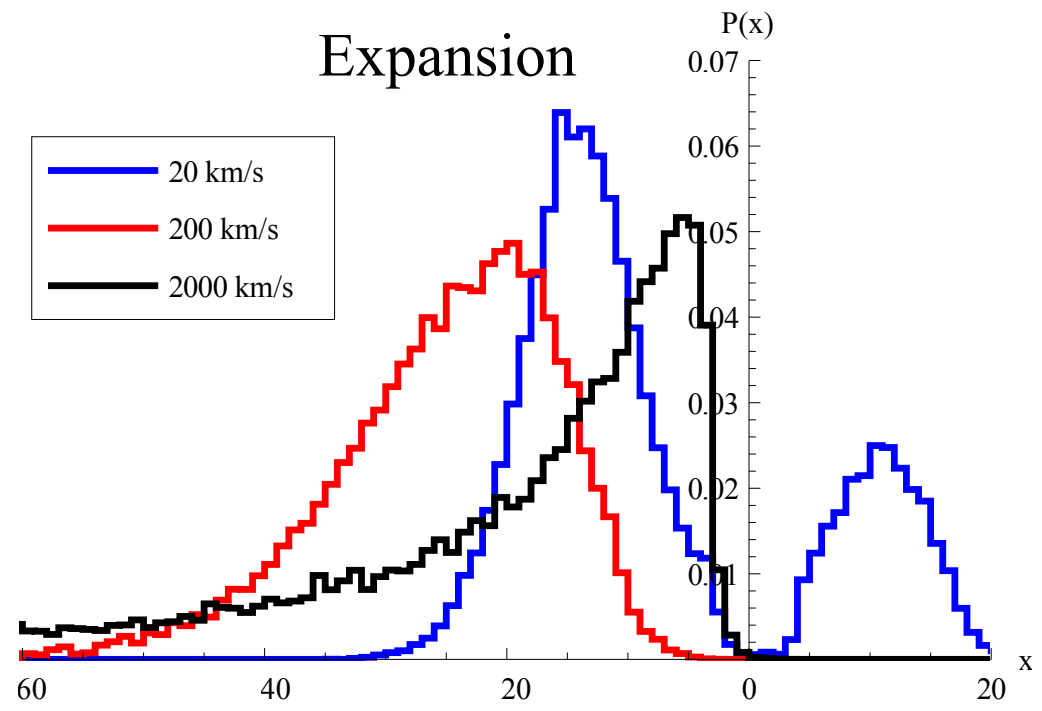

Figure 3.2: Spectra of a homogeneous, isothermal sphere with Lyman- $\alpha$ photons launched at the center and with a frequency $x=0$. Compared to figure 3.1, a radial velocity field has been added, given by $v_{r}=\frac{r}{R} v_{\max }$. The lines correspond to different choices of $v_{\max }$ as indicated in the legend. Taken from Behrens 2011. 
probability to escape. In short, the optical depth for photons moving outwards at at frequency of $+x$ and $-x$ is not the same as it was in the static case, leading to asymmetry in the spectrum. For higher velocities, the peak at the blue side of the spectrum $(x>0)$ completely vanishes (red line). In the case of inflowing gas that we do not plot here, the situation is exactly reversed, and the resulting spectrum is the same as for the expanding case except for a transformation $x^{\prime}=-x$. Although these models are far from realistic, the observation that inflowing and outflowing material tendy to leave a signature in the red or blue part of the spectrum is a robust statement. Spherical models are typically parametrized by the neutral hydrogen (and/or dust) column density $N_{H}$ ( $N_{D}$ for dust), the temperature of the gas $T$ and, in case of a radial velocity field, by $v_{\max }$ as introduced above.

A different class of models, the so-called shell model, was originally presented by Ahn et al. 2003 and further studied by e.g. Verhamme et al. 2006 and Wang 2009. It is well-known that star-forming regions frequently feature an expanding shell of neutral gas (e.g. Chakraborti \& Ray 2011; Heiles 1979; Heiles 1984; Suad et al. 2014). The stellar feedback of young, heavy stars originating from a recent star burst produces an expanding hot gas bubble, ionizing gas within this so-called HII bubble and pushing the outer, neutral material outwards. Since such regions are also expected to emit strong Lyman- $\alpha$ radiation, it is natural to investigate models of such an expanding shell with Lyman- $\alpha$ photons emitted from the center. The shell again is typically assumed to be spherical, and so the system can parametrized by the radial gas column density $N_{H}$, the temperature $T$ of the gas, the thickness of the shell $d$, and the inner radius of the shell $r_{i}$. In figure 3.3, an illustration of such a setup is shown. Additionally, one needs to specify the expansion velocity $v_{\text {exp }}$ that is assumed to be constant in the shell. A typical spectrum of such a shell model is shown in figure 3.4. It shows its highest peak in the red part of the spectrum, which is consistent with the fact that the gas is outflowing (marked by the vertical line). But the mechanism that leads to this distinct peak is different from the sphere models described above: Here, photons that scatter off the inner boundary of the shell escape efficiently if they are scattered by an angle close to $180^{\circ}$, because this induces a large change of their frequency proportional to the outflow velocity. As described by Verhamme et al. 2006, the peak position is related to the outflow velocity: The frequency shift gained by scattering off the inner boundary has its maximum for a scattering angle of $180^{\circ}$ with a shift of twice the outflowing velocity over the speed of light(see equation 1.9 ), which translates into $\Delta x \approx-15$ in the 
case shown in figure 3.4. This so-called Doppler boost leading to efficient escape of Lyman- $\alpha$ photons is of particular interest for the cosmological era prior to (complete) reionization, since the escaping, redshifted photons may penetrate the surrounding, neutral IGM without further scattering. If this model is a good approximation, galaxies at redshifts as high as $z \sim 10$ might be observable in Lyman- $\alpha$ with the next generation of instruments, e.g. JWST ${ }^{1}$ (Dijkstra 2010; Dijkstra 2014; Dijkstra \& Wyithe 2010; Dijkstra et al. 2011).

Verhamme et al. 2008 and Schaerer \& Verhamme 2008 fit shell models to real observational data of Lyman Break Galaxies (LBGs) and LAEs, also introducing an additional dust column density as a model parameter. They identify variations in the hydrogen column density as the main driver of the differences in the observed spectra, and they successfully reproduce the spectra of some well-studied objects using existing knowledge about physical properties of these objects (also see Vanzella et al. 2010). They also published the resulting spectra for a grid of models in parameter space (Schaerer et al. 2011). Verhamme et al. 2014 discuss two different model types for HII shells around star-forming regions in the context of Lyman continuum (i.e., ionizing radiation with wavelengths $<912 \AA$ ) leakers. While the first is simply an isotropic, low optical depth variation of the isotropic shell model, the second model features a clumpy shells with covering factors of less than 1 (see below for more publications on clumpy models). Their main conclusion is that if the spectrum of an LAE shows a peak with a small shift from the line center, the probability for this emitter to leak Lyman continuum radiation is enhanced. They propose to test this hypothesis on several known LAEs.

In the more recent literature, there exists some debate on the extent to which such shell models are able to reproduce observed spectra of LAEs. For example, Kulas et al. 2012 identify several different groups of spectra, using an observed sample of LAEs at $z=2-3$ and find that while shell models qualitatively match some of these groups, they fail to fit the spectra and the parameters derived from other observations of the objects at the same time. In particular, the gas temperature and/or the outflow velocities from fitting the spectra do not match the observed values. One can also argue that there is a degeneracy between the column density and the outflow velocity parameters in the shell model: Low column densities can mimic high outflow velocities and vice versa, especially at low spectral resolution of

1 James Webb Space Telescope 


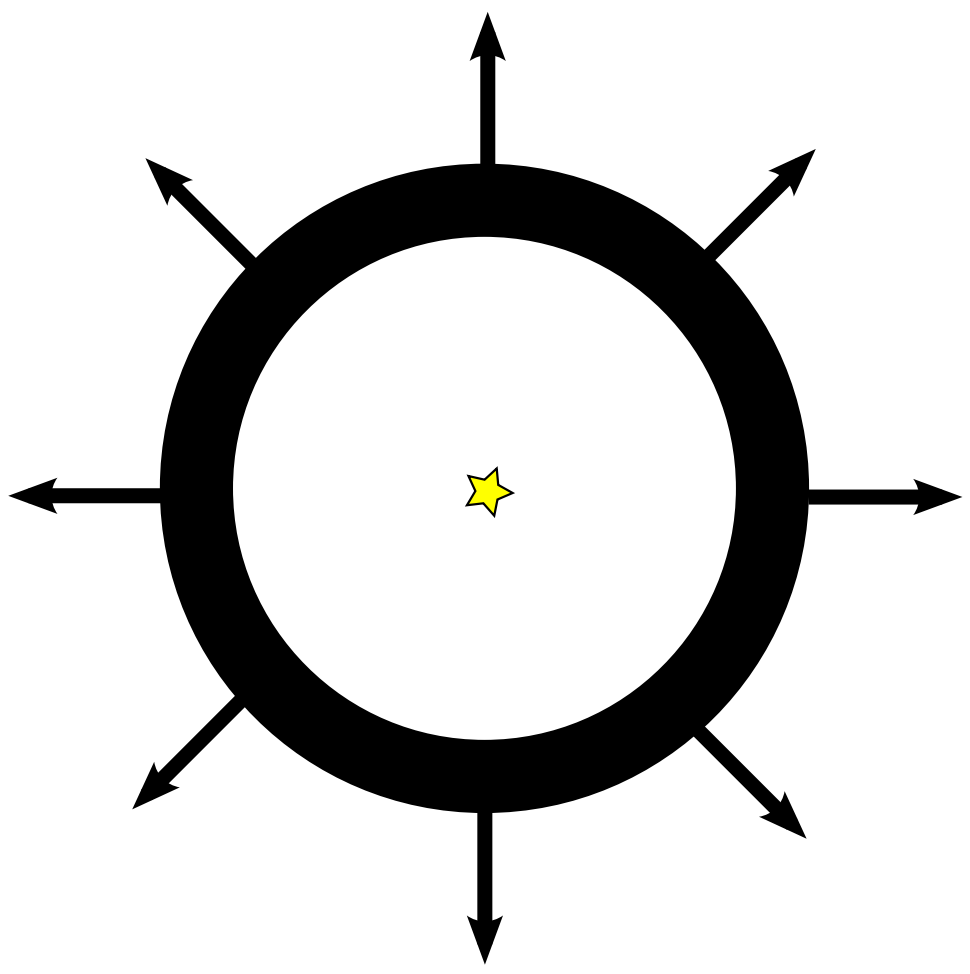

Figure 3.3: Shown is an illustration of the shell model. The black ring represents a slice through the hydrogen/dust shell moving outwards. Photons are emitted in the center.

observations (e.g. Chonis et al. 2013). McLinden et al. 2011 additionally find that for their observed spectra at $z=3.1$, the relative shifts of the red and the (smaller) blue peak is inconsistent with the shell models.

Apart from these considerations, shell models implicitly assume that the expanding $\mathrm{H} 2$ region exhibits spherical symmetry, an assumption that is not realistic. In particular, it is known from observations that specific lines of sight with a lower-thanaverage optical depth exist, for example due to stellar feedback blowing a 'hole' in the gas distribution (e.g. Chonis et al. 2013). Anisotropies of this kind could also help to understand the large equivalent widths (EWs) found for some LAEs, exceeding $500 \AA$ which is inconsistent with the observed initial mass distribution (Zheng et al. 2014). This could be explained if Lyman- $\alpha$ photons preferentially escape along some lines of sight, boosting the number of photons observed in these directions.

Before turning to the research done on anisotropic, simplified models of LAEs, we briefly review a different family of models that we label here as 'clumpy' models. 


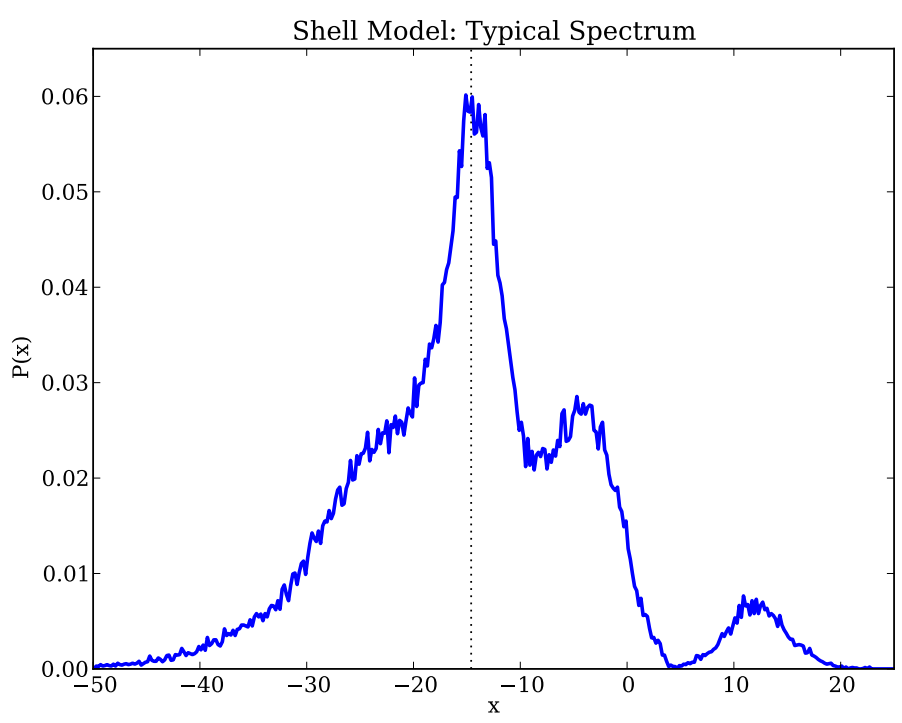

Figure 3.4: Spectrum of a shell model with parameters $N_{H}=10^{20.2} \mathrm{~cm}^{-2}, T=10^{5} \mathrm{~K}$, $v_{\text {exp }}=300 \mathrm{~km} / \mathrm{s}$, monochromatic point source, no dust. The vertical line marks the typical red peak showing up for this type of model. It results from photons that are scattered at the inner boundary of the shell back into the interior, resulting in a frequency shift that reduces the optical depth.

These models have in common that they do not model the ISM as a continuous sphere or shell, but in terms of a distribution of clouds or clumps of gas. This is mainly motivated by the fact that the ISM is known to be clumpy, with dense, cold clouds embedded in a more diffuse, hot phase (e.g. Hennebelle \& Falgarone 2012), and by the so-called Neufeld scenario (Neufeld 1991). This scenario suggests that Lyman- $\alpha$ photons can escape from a clumpy, dusty medium more easily than from a homogeneous medium with (formally) similar optical depths under certain conditions. In particular, it suggests that the effective optical depth for Lyman- $\alpha$ photons is drastically reduced with respect to the homogeneous case, whereas the optical depth for continuum photons stays about the same. The key idea here is that Lyman- $\alpha$ photons simply bounce off the boundary of the dense clouds due to their very large scattering cross section (compared to the continuum photons), while continuum photons penetrate the clouds, rendering their absorption by dust in these clouds probable. Lyman- $\alpha$ photons do not face absorption because they rarely enter the clouds. They scatter on the surface of various clouds until they leave the ISM. An illustration of such a model and the Neufeld scenario is shown in figure 3.5. 


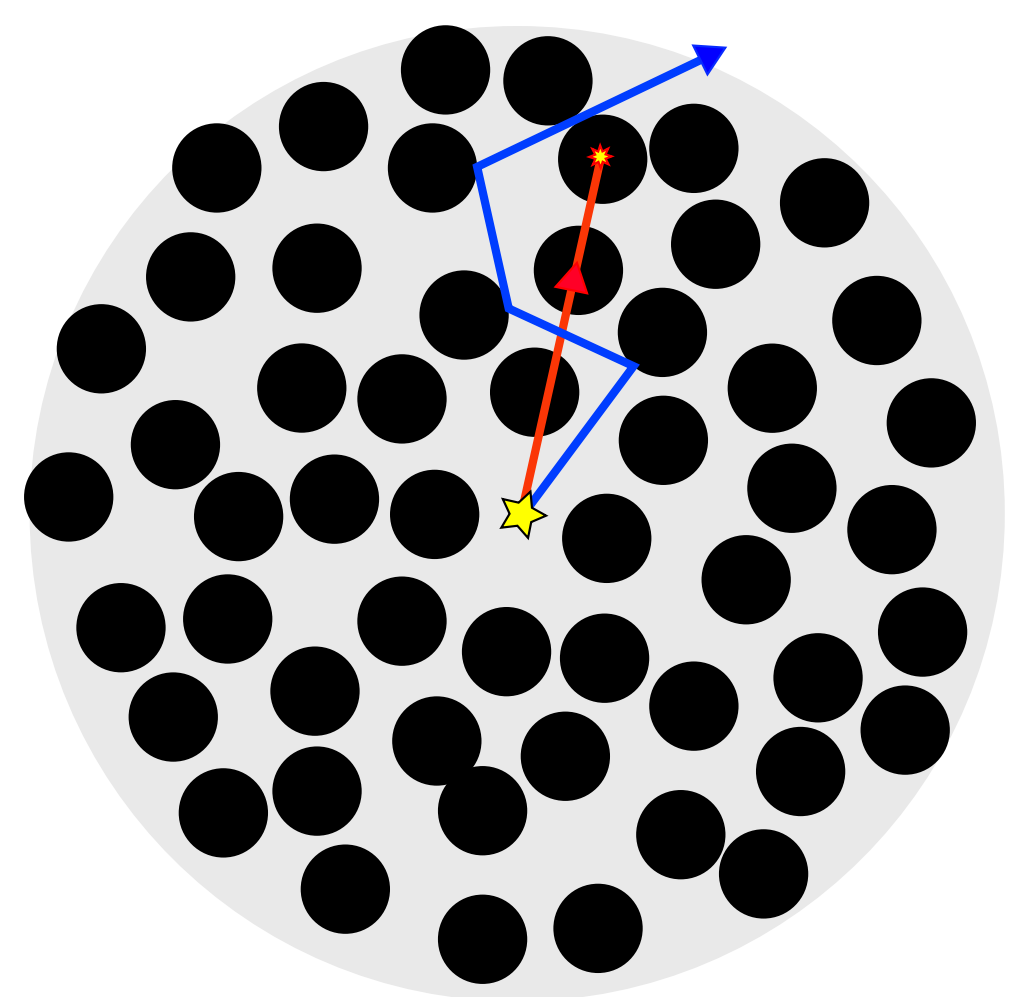

Figure 3.5: Illustration of a clumpy LAE model. Shown is a slice. The grey area represents the low-density, high-temperature intercloud medium, while the black circles represent dense, dusty clouds. Photons are emitted in the center (yellow star). The Neufeld scenario is illustrated by the two rays. The red one represents the path of a continuum photon. Due to its small cross section with hydrogen, it penetrates the clouds on its way out and therefore is attenuated by dust, and might finally be absorbed. The Lyman- $\alpha$ photon (depicted with a blue line) has a large cross section and therefore rarely enters the clouds. Instead, it bounces off the boundaries of the clouds and leaves the region. As described in the text, this mechanism works only under certain conditions. Note that clouds can have individual velocities, and the emission region can be extended.

Several authors analyzed models inspired by this scenario, e.g. Haiman \& Spaans 1999; Hansen \& Oh 2006; Richling 2003, and Surlan et al. 2013. Shimizu et al. 2012 used a simple subgrid model to include this effect in cosmological simulations. However, Duval et al. 2014 and Laursen et al. 2013 show that the conditions under which the Neufeld scenario actually boosts the Lyman- $\alpha$ escape fraction are quite special. The velocity dispersion of the clouds must be very low $(<10 \mathrm{~km} / \mathrm{s})$, the hot phase of the ISM must have densities $<10^{-7} \mathrm{~cm}^{-3}$, the embedded clouds need to have densities well above the observational values, and outflows, if existing, are 
only allowed to have velocities of $<10 \mathrm{~km} / \mathrm{s}$. Laursen et al. 2013 and Duval et al. 2014 therefore conclude that these conditions will be rarely met in the ISM. Apart from the failure of the Neufeld scenario in explaining the observed EW excess, the clumpy models typically do not show spectra that are qualitatively different from the continuous (shell) models.

\subsection{Anisotropic Simplified Models}

As already mentioned above, the fact that the ISM is known to be clumpy and open questions like the one for the origin of the high-EW LAEs led several groups to consider anisotropic models. Whereas the models described in the preceding section all had spherical symmetry (at least approximately in the case of the clumpy models $)^{1}$, the models considered here deviate strongly from spherical symmetry. Zheng \& Wallace 2014 discuss spherical gas distributions like the sphere models discussed above, but with a deviation from spherical symmetry in velocity structure and/or density structure along the $z$-axis, i.e. the models remain axisymmetric, and the resulting anisotropic Lyman- $\alpha$ properties can be investigated in terms of the angle $\theta$ between the line of sight of an observer and the $z$-axis. In particular, they discuss the so-called density gradient models where the local hydrogen number density $n$ is given by

$$
n(z)=\bar{n}\left(1-2 A \frac{z}{R}\right)
$$

with $\bar{n}$ the mean number density, $R$ the radius of the sphere and $A$ the asymmetry parameter. $A=0$ corresponds to the isotropic case. For $A=0.5$, the density becomes zero at $z=R$. As expected, such a model introduces an inclination dependency of the flux and spectrum. For example, for a value of $A=0.5$, they find that the flux escaping along the positive $z$-axis is 2.4 times larger than the flux escaping towards $-z$ (in this case, the typical column density is $N_{H}=\bar{n} R=10^{19} \mathrm{~cm}^{-2}$ ). We show the flux as a function of angle in figure 3.6 (left panel). Assuming an intrinsic EW of the Lyman- $\alpha$ radiation and further assuming that the continuum radiation is unaffected by the gas due to its low cross section, they calculate the resulting distribution of EWs as a function of angle of observation, and find that the distribution is broadened

1 Some clumpy models discussed above, e.g. employing a small number of large clouds, have intrinsically anisotropic Lyman- $\alpha$ properties. However, the publication mentioned above did not analyze these anisotropies explicitly, but integrated the spectrum over all lines of sight. 
towards high values for large asymmetry parameters $A$. This is natural, since some observers along the $+z$ axis see up to a factor of 1.7 more Lyman- $\alpha$ photons than expected for the isotropic case. As a consequence, these observers also see a EW higher than the intrinsic EW by a factor of 1.7. The spectrum is double-peaked for these models since the gas is static, and there is a correlation between the offset of the peaks from the line center, the widths of the peaks and the EW. This is explained by the fact that photons escaping through regions with reduced column density are not shifted as far into the wings as photons escaping from the high-density regions.

As a second type of model, they discuss models with a velocity gradient. The velocity field at a location $\vec{r}$ is given by:

$$
\vec{v}(\vec{r})=\frac{r}{R} V \vec{e}_{r}+\frac{z}{R} \Delta V \vec{e}_{z+}
$$

In this equation, $\vec{e}_{r}$ denotes a unit vector pointing radially outwards, $\vec{e}_{z+}$ is a unit vector along the $+z$ axis, $V$ and $\Delta V$ are constant velocities. The first term describes an isotropic expansion and is identical to the prescription for the original sphere models described in the previous section. The second term introduces a deviation from spherical symmetry in terms of an additional inflow/outflow towards the $\pm z$ axis parametrized by $\Delta V$. Due to the velocity field, the optical depth along the $\pm z$ axis is reduced. We show the resulting flux in figure 3.7 (left panel). As expected, this results in a boost in flux of about a factor of 2 (for $\Delta V=200 \mathrm{~km} / \mathrm{s}$ ) and EWs up to a factor of 2 higher for observer along the $\pm z$ axis. Again, they find a correlation
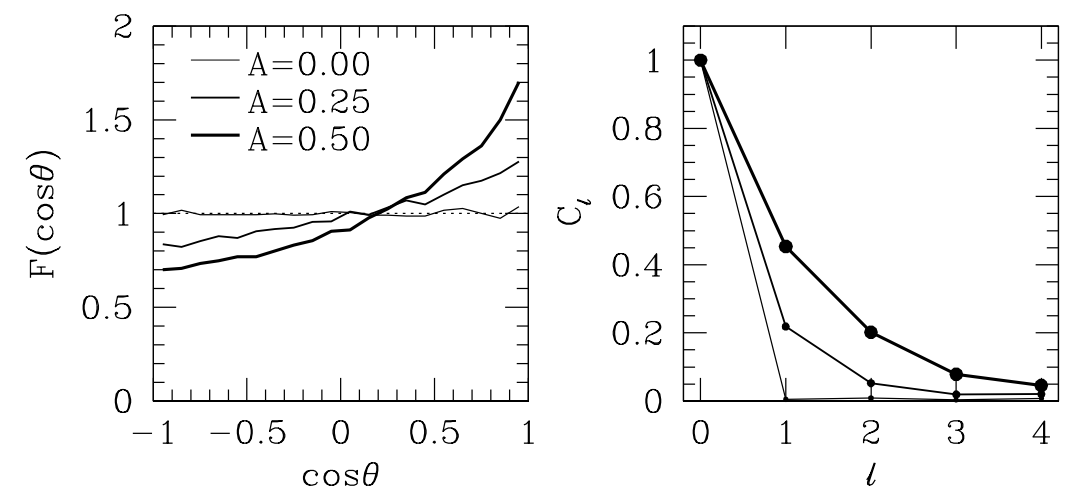

Figure 3.6: Left: flux as a function of angle of observation for the 'density gradient' model for three different values of $A$. The flux is normalized to the isotropic case. Right: coefficients of the multipole expansion of the emerging flux distribution. Reprinted with permission (C) AAS (Figure 2 in Zheng \& Wallace 2014) 

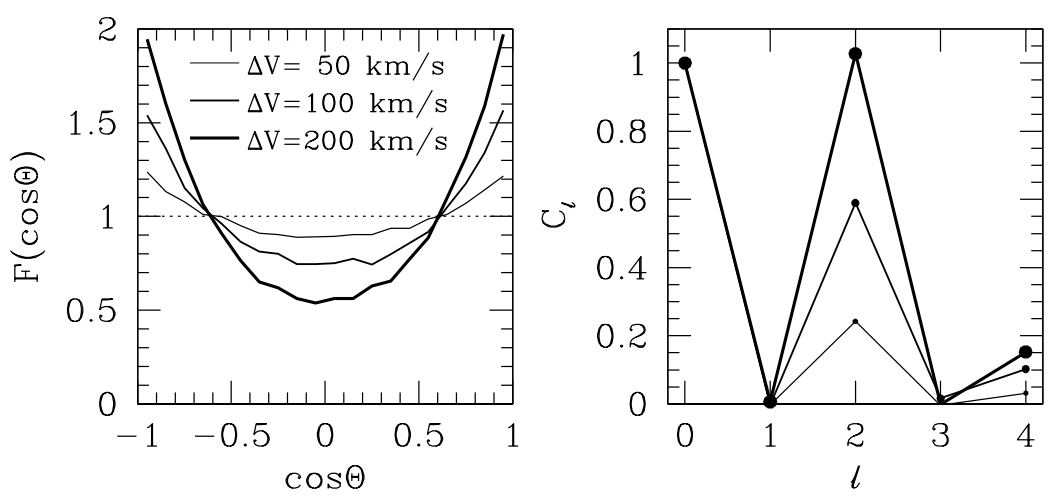

Figure 3.7: Left: flux as a function of angle of observation for the 'velocity gradient' model for three different values of $A$. The flux is normalized to the isotropic case. Right: coefficients of the multipole expansion of the emerging flux distribution. Reprinted with permission (C) AAS (Figure 6 in Zheng \& Wallace 2014)

between the EW that is observed along a certain line of sight and the peak shift.

As a third family of models, Zheng et al. 2014 present a case where a spherical gas cloud undergoes expansion only within a certain solid angle, i.e. an area of the form of a cone with a certain opening angle $\Theta$ is expanding, while the rest of the gas remains static. Within a cone defined by $|z| / r<\cos \Theta$, the velocity field is given by

$$
\vec{v}(\vec{r})=\frac{r}{R} V \vec{e}_{r}
$$

and zero otherwise. For $V=0$, this is again reduced to the isotropic (static) case. This is called the 'bipolar wind' model, because it is motivated by the existence of bipolar outflows in galaxies (e.g. Rubin et al. 2013). The results in terms of the EW as a function of angle of observation are similar to the velocity gradient case. The spectra are similar to a static spherical problem when observed perpendicular to the $z$-axis, but resemble the expanding sphere when observed along the $z$-axis.

Behrens et al. 2014 (see section 4.2) further investigate anisotropic models, but focus on modifications of the originally isotropic shell models that were described in the previous section. The motivation here is not only to understand the features of Lyman- $\alpha$ spectra and EW distributions, but also to find a relation between the Lyman- $\alpha$ transmission and the observability of Lyman-continuum photons (also see Verhamme et al. 2014, as described above).

We consider again a point source for the Lyman- $\alpha$ photons, but with a finite width in frequency space given by a Gaussian. As a first modification, we consider isotropically expanding shells with a conical region removed at the top and bottom 
(which we define to be the $\pm z$ direction, see figure 2 in section 4.2 for an illustration). In addition to the parameters of the shell model, this introduces only one new parameter, namely the solid angle subtended by the cone, $\Omega$, that we can also parametrize by the opening angle of the cone, $\alpha$. The flux variation as a function of angle of observation $\Theta$ (again defined as the angle between the $z$ axis and the line of sight) is approximately constant for angles smaller than $\alpha$, jumps to a lower value above $\alpha$ and remains approximately constant afterwards: Below $\alpha$, observers see the unscattered photons streaming through the hole at the top/bottom. Above that angle, photons are scattered by the shell, and some are scattered into lines of sight that lead to escape through the holes. Therefore, the flux at these inclinations is reduced (see figure 7 in section 4.2). The spectrum at large angles resembles the shell spectrum (see figure 3.4), but observers looking down the carved-out hole at the top/bottom see a large peak at the line center, i.e. the unscattered, initial Gaussian. Width and height of this peak depend on the width of the Gaussian. For typical column densities, observers looking down the holes see up to a factor of $\sim 1.7$ (6 when dust is included) more Lyman- $\alpha$ flux than expected from isotropic escape. Under the assumption that continuum photons are not affected by the gas shell, this means also a boost in observed EW by the same factor.

As a second class of models, Behrens et al. 2014 introduce the bipolar shell models. They are different from the bipolar models presented by Zheng et al. 2014 in the sense that they are again based on the shell model and that the density distribution is actually deformed by a non-spherical velocity field. The velocity field is given by

$$
\vec{v}(\vec{r})=\vec{e}_{r}\left(v_{b} \cos \Theta+v_{c}\right)
$$

where $v_{b}$ parametrizes the anisotropic velocity component and $v_{c}$ the isotropic expansion. Such a velocity field will deform an initially spherical shell by radially displacing it by a distance $s$ :

$$
s=\left(v_{b} \cos \Theta+v_{c}\right) t
$$

The density field evolves accordingly. In particular, with increasing time $t$ the shell becomes less dense due to its expansion (because mass is distributed in a shell of identical thickness, but larger radius), and the drop in density occurs faster in regions at low $\Theta$ because these regions expand faster. An illustration of different evolutionary 
stages of this type of model is given in figure 1 in section 4.2. As is evident from equation 3.7, for $v_{b}=0$ this is the original, isotropically expanding shell model. Typically, the variation in flux here is less drastic and more continuous than in the shell with holes model. The reason is that the change in column density is not as abrupt as in the shell with holes case, and that the change is not as large (see figure 4, section 4.2 for a detailed comparison of the column density as a function of angle). The expected EW boost from this class of models is only few $\sim 10 \%$. An example for the Lyman- $\alpha$ properties of these models is shown in figure 5 and 6 in section 4.2. The spectra differ depending on whether we investigate the 'early' $(t=0)$ or 'late' case $(t \sim 0.1)$ : In the early case, the spectra are asymmetric, double-peaked, and look similar along all lines of sight. In the late case, the optical depth for photons escaping along the $\pm z$ axis is so low that observers located in these direction can see parts of the intrinsic gaussian spectrum as a peak at the line center. However, the peak is not as dominant as in the shell with holes models, and it is not distinguishable from the rest of the spectrum if the gaussian input spectrum is wide $(>100 \mathrm{~km} / \mathrm{s})$ so that the peak merges with the rest of the spectrum. Nevertheless, we note that similar to the shell with holes model, there is a correlation of a peak at the line center and the line of sight having relatively low optical depth.

As a third family of models, Behrens et al. 2014 investigate the so-called cavity models. These are the only class of models that have no direct connection to the shell model, and can be thought of as an extreme version of a late stage of a bipolar wind in a galaxy. While the wind has cleared a cavity along the $\pm z$ axis, there is a ring-like, homogeneous gas distribution around it, here defined by the intersections of two parabolae. Illustrations of such models are given in figure 3, section 4.2. Additionally to the anisotropic density distribution, we introduce a linear velocity field described by

$$
\vec{v}(z)=\vec{e}_{z} \frac{z}{z_{\max }} v_{l}
$$

where $v_{l}$ parametrizes the maximum velocity at the tip of the ring-like density structure with $z$-coordinate $z_{\max }$.

As for the shell with holes models, the cavity models have a large total change in column density as a function of $\Theta$ due to the cleared cavity in the center, but the change does not occur abruptly. The resulting flux variation with angle boosts the EW typically by a factor of about 3 for lines of sight that pierce through the 
cavity, independent of the inclusion/exclusion of dust (see Figure 8 in section 4.2 for an example). The flux variation resembles the variation in the shell-with-holes case, with the modification that the transition is smooth, as expected. The spectrum has a complicated shape compared to the other models. For lines of sight through the cavity, it is double-peaked and asymmetric, and if the intrinsic line width is small $(<100 \mathrm{~km} / \mathrm{s})$, it shows a third peak at the line center. As $\Theta$ grows larger, the blue peak in the spectrum vanishes. Above the effective opening angle of the inner cavity, the distinct peaks vanish.

Similar to Zheng et al. 2014, Behrens et al. 2014 find correlations between the observed EW and the position of the peak in the spectrum. Behrens et al. 2014 quantify this by running a parameter study for the three families of models, varying the column densities, velocity parameters and - in the case of the cavity models - the shape of the gas rings (see table 2 in section 4.2 for a detailed list of parameters). The main result is that the probability of observing a peak at the line center is increased by a factor of a few along lines of sight that pierce through the regions of low optical depth (see figure 13 in section 4.2). In turn, this implies that the probability of finding emitters leaking Lyman continuum radiation is increased if the emitter is an LAE with an emission peak near the line center, or an flux excess near the line center. This result is in accordance with Verhamme et al. 2014 for isotropic models. However, our results apply also in the regime of high column densities in the presence of significant anisotropy, whereas Verhamme et al. 2012 are restricted to low effective column densities.

Recently, Gronke \& Dijkstra 2014 presented a set of clumpy Lyman- $\alpha$ models. In contrast to earlier work on clumpy models (see previous section), they focus on the directional dependence of the resulting Lyman- $\alpha$ properties due to the existence of individual lines of sights with low optical depth. These models are different from the others described in this section in the sense that they do not have an axisymmetric setup, i.e. there is no generally preferred direction of escape (in the work previously presented, this was typically the $\pm z$ axis). Instead, lines of sight with low optical depth arise at random angles $(\phi, \Theta)$ owing to the clumpy structure of their setup with low average covering factors. Their models are inspired by Laursen et al. 2013 who did not consider the directional dependence explicitly. Gronke \& Dijkstra 2014 define the boosting factor $b$ as a parameter connecting the observed EW along a 
specific line of sight with the intrinsic EW,

$$
E W_{\text {observed }}=b \times E W_{\text {intrinsic }}
$$

In these terms, Laursen et al. 2009 only calculated the average $\bar{b}$ by averaging over all lines of sight. Gronke \& Dijkstra 2014 follow Laursen et al. 2013 in assuming values for the density and temperature for the intercloud medium and the clouds, but focus on the distribution of boosting factors. The velocity of the clouds is given by a radial component modeling the outflow and a random contribution given by a velocity dispersion $\sigma_{v} \sim 10-40 \mathrm{~km} / \mathrm{s}$ :

$$
\left.\vec{v}(r)=v_{\max }\left(1-\frac{r}{r_{\min }}\right)^{1-\alpha}\right)^{0.5} \vec{e}_{r}+\vec{v}_{\text {random }}\left(\sigma_{v}\right)
$$

with $r_{\text {min }}$ the minimum distance from the center (the first term is set to zero for $\left.r<r_{\text {min }}\right)$. $\alpha$ controls the acceleration of the clouds with radius. While they reproduce the average boosting factor $\bar{b}$ from Laursen et al. 2013, they find broad variability of $b$ from line of sight to line of sight. For example, for their 'fiducial' parameter set, they find about $10 \%$ of the lines of sight to have $b>3 \bar{b}$ and about $1 \%$ with $b>7 \bar{b}$. In figure 3.8, we show the relative enhancement in Lyman- $\alpha$ (continuum) escape fraction in the upper left (upper right) panel as a function of $(\phi, \Theta)$. The resulting boost in EW is shown in the lower left panel, while the lower right panel shows the distribution of boost parameters $b$. However, a more realistic choice of parameters (their 'realistic' parameter set) yields variations that are consistent with Poisson noise, indicating that the statistical anisotropies are too small to have significant effect (see figure 3.9 for a plot of the resulting boost). They argue that this is in part due to the inefficiency of the Neufeld scenario even for individual lines of sight, but also due to the fact that in the realistic scenario, the emitting region is assumed not to be point-like but extended, averaging out random directional fluctuations of the optical depth for continuum photons and consequently in the EW distribution. 

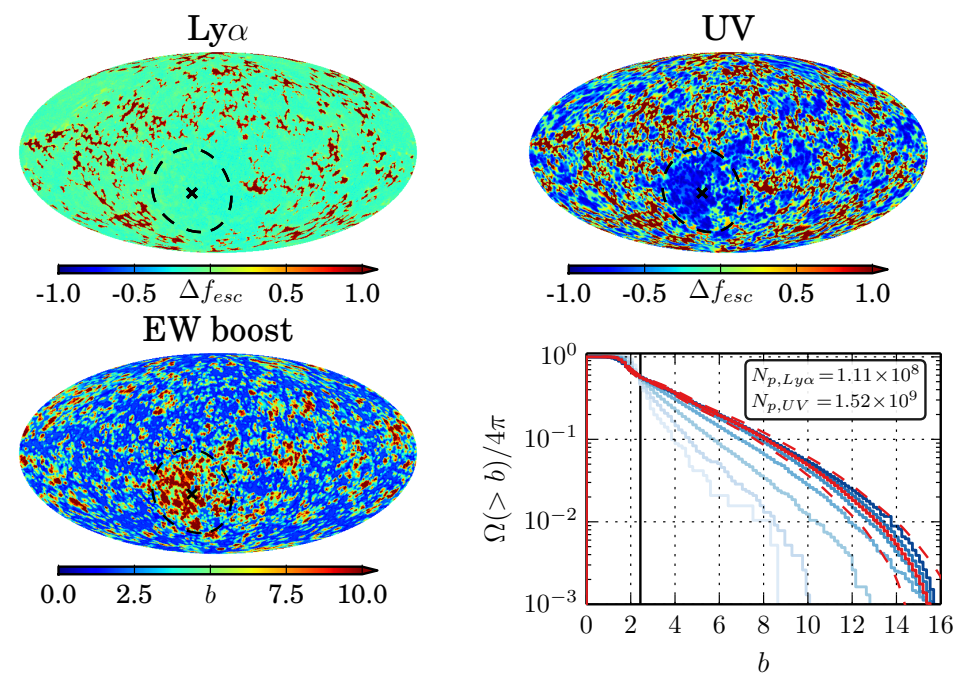

Figure 3.8: Results for the "fiducial" parameter set of Gronke \& Dijkstra 2014. Upper left: projected relative enhancement of Lyman- $\alpha$ escape fraction as a function of the angles $(\phi, \Theta)$. Upper right: projected relative enhancement of continuum escape fraction as a function of the angles $(\phi, \Theta)$. Lower left: projected EW boost $b$ as a function of the two angles. Lower right: cumulative Distribution of $b$ in fractions of sky coverage. The different lines show the result for different sizes of the angular bins, with the darker lines corresponding to smaller bins. The solid vertical line shows the average $\bar{b}$. The red lines show the distribution for the resolution that was used in the other panels, with the dashed lines showing the standard deviation. Reprinted with permission (Figure 1 in Gronke \& Dijkstra 2014)
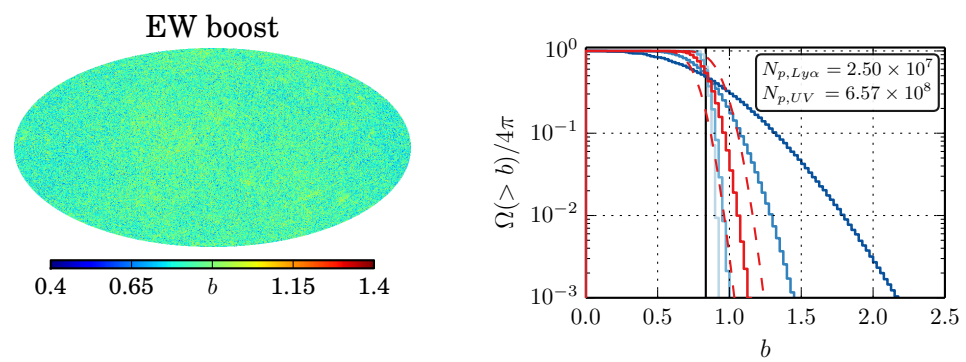

Figure 3.9: Results for the "realistic" parameter set of Gronke \& Dijkstra 2014. Left: projected EW boost $b$ as a function of $(\phi, \Theta)$. Right: cumulative Distribution of $b$ in fractions of sky coverage. The different lines show the result for different sizes of the angular bins, with the darker lines corresponding to smaller bins. The solid vertical line shows the average $\bar{b}$. The red lines show the distribution for the resolution that was used in the other panels, with the dashed lines showing the standard deviation. Reprinted with permission (Figure 2 in Gronke \& Dijkstra 2014) 


\subsection{Towards Realistic LAEs}

While the previous section considered simplified models with only few parameters and a more or less simple geometry, the models in this section have in common that they all aim for more realistic and therefore morphologically and geometrically complicated models of LAEs. They are typically based on the outcome of hydrodynamical simulations of individual galaxies or small cosmological volumes, focusing on a small group of galaxies. Different from the publications presented in 2.1 which marginally resolve the ISM at best, the publications summarized here typically have high resolutions of 500-18 pc. We start by considering work on small cosmological volumes and turn to isolated galaxies later.

\subsubsection{Models Based on Cosmological Simulations}

Tasitsiomi 2006 present results from a zoom-in simulation of an individual halo (stellar mass $10^{10} \mathrm{M}_{\odot}$ ) with an effective resolution of $29 \mathrm{pc}$ at $z \sim 8$ in a small cosmological volume. They include the transfer of Lyman- $\alpha$ photons, but no continuum radiation and no dust. The source of the Lyman- $\alpha$ photons is assumed to be predominantly recombination after ionization by Lyman continuum photons from young, hot stars. They inject the Lyman- $\alpha$ photons spatially distributed in the cells that have an
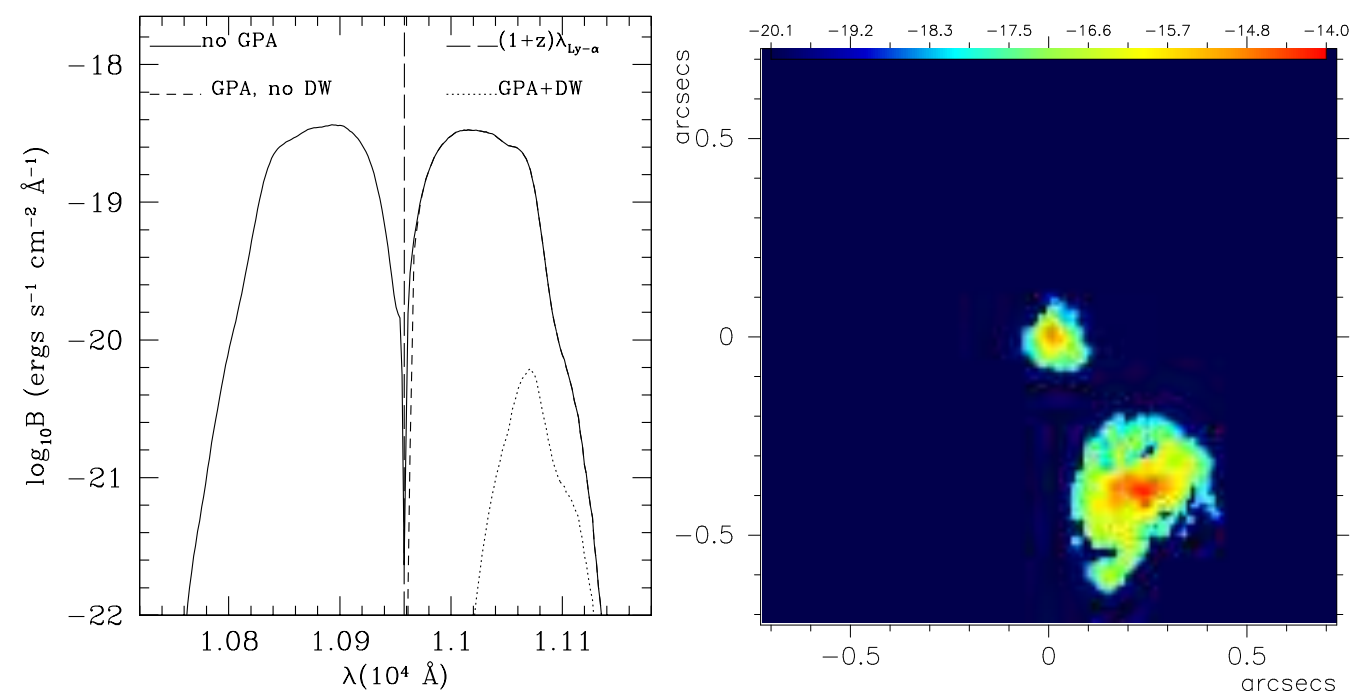

Figure 3.10: Left: spectrum of the emitter studied by Tasitsiomi 2006, with (dotted) and without (solid line) attenuation by the neutral IGM. The dashed line shows the spectrum with attenuation of IGM only in the blue part of the spectrum, the long-dashed line indicates the rest-frame line center. Right: surface brightness map of the emitter. Reproduced by the permission of the AAS (Figure 10 in Tasitsiomi 2006) 
intrinsic luminosity above a certain threshold to reduce computation time. They calculate the emerging spectrum integrated over all lines of sight, and find it to be nearly symmetric and double-peaked with hints of a slightly enhanced blue peak resulting from gas streaming into the halo as explained in the first section of this chapter. We show the spectrum emerging from the emitter in figure 3.10 (solid line). To simulate observations of an object at this redshift, one has to correct for the effect of the neutral hydrogen between the object and the observer. Before complete reionization, this is called the Gunn-Peterson trough (Gunn \& Peterson 1965) known from quasar spectra (e.g Becker et al. 2001). Effectively, it scatters all photons bluewards of the line center out of the line of sight since the Hubble flow will shift these photons in resonance with the neutral IGM after traveling some distance from the emitter. Partially, this will also occur for the red side of the spectrum due to the (thermal) motions of the intervening gas. The attenuated spectrum is shown in figure 3.10 (dotted line). Tasitsiomi 2006 note that the effect of the inflowing gas on the shape of the spectrum is probably quite low because the column densities are so high that a velocity shift of a few hundred $\mathrm{km} / \mathrm{s}$ does not significantly reduce the optical depth for blue photons.

Laursen et al. 2009 present a set of 9 zoom-in simulations ${ }^{1}$ of halos taken from
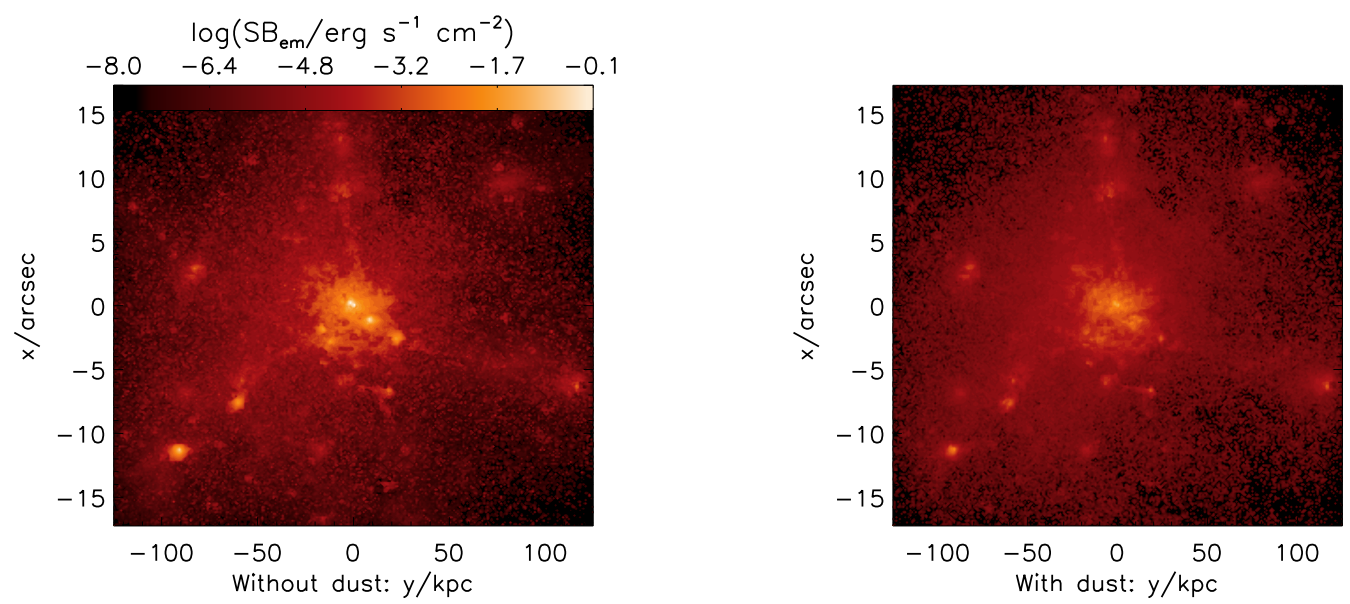

Figure 3.11: Surface brightness map for one of the emitters studied by Laursen et al. 2009. Left: excluding dust effects. Right: including dust effects. Reprinted with permission (c) AAS (Figure 4 in Laursen et al. 2009)

1 We skip the discussion of Laursen \& Sommer-Larsen 2007 since Laursen et al. 2009 directly extends this work. Laursen \& Sommer-Larsen 2007 present calculations for one LAE without dust. 


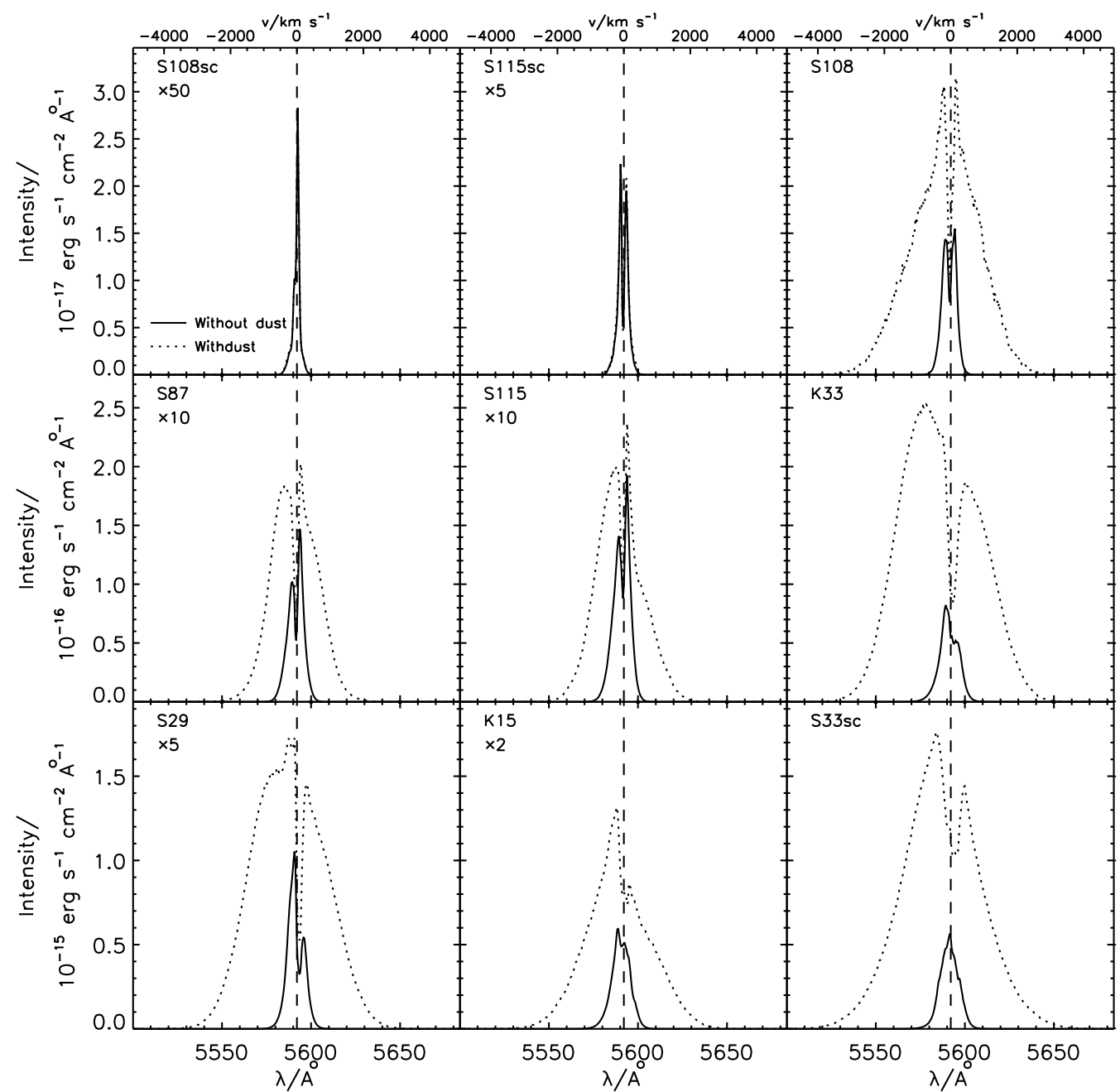

Figure 3.12: Spectra of the emitters studied in Laursen et al. 2009 for both a dust-free case (dotted) and including dust (solid lines). Reprinted with permission (c) AAS (Figure 10 in Laursen et al. 2009)

a cosmological volume with stellar masses of $6 \times 10^{6}-3 \times 10^{10} \mathrm{M}_{\odot}$ and effective resolutions of 491 to $137 \mathrm{pc}$ at $z=3.6$. They do not only include Lyman- $\alpha$ photons from recombination, but also from gravitational cooling and the UV background. However, $90 \%$ of the Lyman- $\alpha$ photons are generated by recombination of Lymancontinuum photons emitted by young stars. In figure 3.12, we show the resulting spectra from all nine studied emitters, both including effects of dust on the radiative transfer (solid lines) and excluding it (dotted lines). They find the counter-intuitive result that while the dust cross section for Lyman- $\alpha$ photons is independent of frequency, the effect of dust on the emerging spectrum is not: Dust tends to remove 
more flux far away from the line center than near the line center. The reason is the interplay of the absorption by dust with the scattering on neutral hydrogen. Photons that diffuse far out into the wings are those residing in dense environments where the optical depth emerging from hydrogen is high. These photons are therefore the ones that are scattered often and have large path lengths, which makes them subject to severe attenuation. On the other hand, photons escaping at the line center are typically located in low-density environments and escape after fewer scatterings, rendering absorption less probable. Additionally, dust is mostly present near the dense regions where it was produced. In figure 3.11, we show a surface brightness plot of the emerging Lyman- $\alpha$ radiation of a single emitter without (left) and with dust (right) for illustration. They find an escape fraction of Lyman- $\alpha$ of about unity for the two emitters with less than $10^{10} \mathrm{M}_{\odot}$ dynamical mass, and about $30 \%$ escape fraction above this mass, with a trend to lower escape fraction for higher mass. Additionally, Laursen et al. 2009 find a dependency of flux on orientation of up to a factor of 4 .

Yajima et al. 2012 present simulations of a cosmological volume with a resolution of $342 \mathrm{pc}$ and simulate radiative transfer for the 10 most massive galaxies at six redshifts between 3.1 and 10.2 with stellar masses of about $10^{10} \mathrm{M}_{\odot}$ at $z=3.1$. They include Lyman- $\alpha$ from both recombination and collisional excitation, continuum radiation, ionizing radiation, and dust. The Lyman- $\alpha$ emissivity here is derived from the ionization state due to the ionizing radiation transport. They find varying EWs $(\sim 67-21 \AA)$ and increasing escape fractions $(\sim 0.13-0.6)$ with decreasing redshift, indicating an evolution of the emitters. The Lyman- $\alpha$ luminosity, however, does evolve only by a factor of 2 . Their spectra are typically single-peaked and slightly bluer than the intrinsic Gaussian spectrum, which they consider to be due to strong ionization near the sources and large outflow velocities.

For completeness, we also mention here the work by Barnes et al. 2011 that focuses on Damped Lyman- $\alpha$ absorbers (DLAs) as a source of extended Lyman- $\alpha$ emission, employing a cosmological zoom-in simulation of 3 objects at a resolution of $514 \mathrm{pc}$ $(z \sim 3)$. They only follow Lyman- $\alpha$ photons (modeled as central point source) and do not include dust. They find the absorption region of the objects to be generally smaller than the Lyman- $\alpha$ emission region, with the central source illuminating neutral clouds further outside. Additionally, they find a dependency of the Lyman- $\alpha$ properties on angle of observation.

It is worth noting that while the publications above partially comment on anisotropic 
escape (e.g Barnes et al. 2011; Laursen et al. 2009; Yajima et al. 2012), they do not focus on it. To investigate the effects of anisotropies in the ISM and disentangling them from effects of the circumgalactic medium (CGM) and IGM, one can use simulations of isolated galaxies.

\subsubsection{Models Based on Simulations of Isolated Galaxies}

Verhamme et al. 2012 present a detailed simulation of an isolated disk galaxy ${ }^{1}$ with a stellar mass of about $6.8 \times 10^{8} \mathrm{M}_{\odot}$ (dust mass $\left.8.7 \times 10^{6} \mathrm{M}_{\odot}\right)$ and a resolution of 18 pc. The hydrodynamical simulation includes cooling from gas and metals, an effective description of the dense ISM by a polytropic equation of state, star formation according to a Schmidt law and stellar feedback from supernovae explosions with metal enrichment. They follow the evolution of the galaxy over a time span of 6 Gyr. Lyman- $\alpha$ and continuum photons are launched at the position of young star clusters $^{2}$. The intrinsic spectrum for the Lyman- $\alpha$ photons is a Gaussian with fixed width $(20 \mathrm{~km} / \mathrm{s}$ FWHM), while the continuum is assumed to be flat in a range of $\pm 2 \times 10^{4} \mathrm{~km} / \mathrm{s}$. The intrinsic EW is set to $200 \AA$, which fixes the ratio of continuum photons emitted per wavelength and the total number of Lyman- $\alpha$ photons. In figure 3.13 , we show where photons escape in their simulation, that is, where they were scattered last before escaping directly (colored contours), the density (grey contours), and the velocity field (arrows). Dust is included in the simulations, and is formed from metals proportional to the neutral hydrogen column density.

The most important finding of their work is the fact that the ISM renders the escape of photons highly anisotropic. Most Lyman- $\alpha$ photons escape face-on, corresponding to an inclination $|\cos \Theta| \approx 1$. This can clearly be seen in figure 3.14 taken from their paper. Here, the red solid line shows the probability of a Lyman- $\alpha$ photons to escape as a function of inclination $|\cos \Theta|$. The probability of escaping face-on is enhanced by a factor of about 15 . The black solid line shows the fraction of photons that are not absorbed and escape, given that the photon was initially emitted with an inclination $|\cos \theta|$. The flat line at $5 \%$ indicates that the average escape fraction is about $5 \%$, and that the probability of escape/absorption is independent of initial

1 In fact, they present a high-resolution and a low-resolution simulation. We focus here on the highresolution simulation (labeled G2 in the paper)

2 In such simulations, the mass resolution is to low to cover individual stars. Instead, so-called star particles are used to trace the stellar mass. These particles can be thought of as tracers of stellar clusters. 

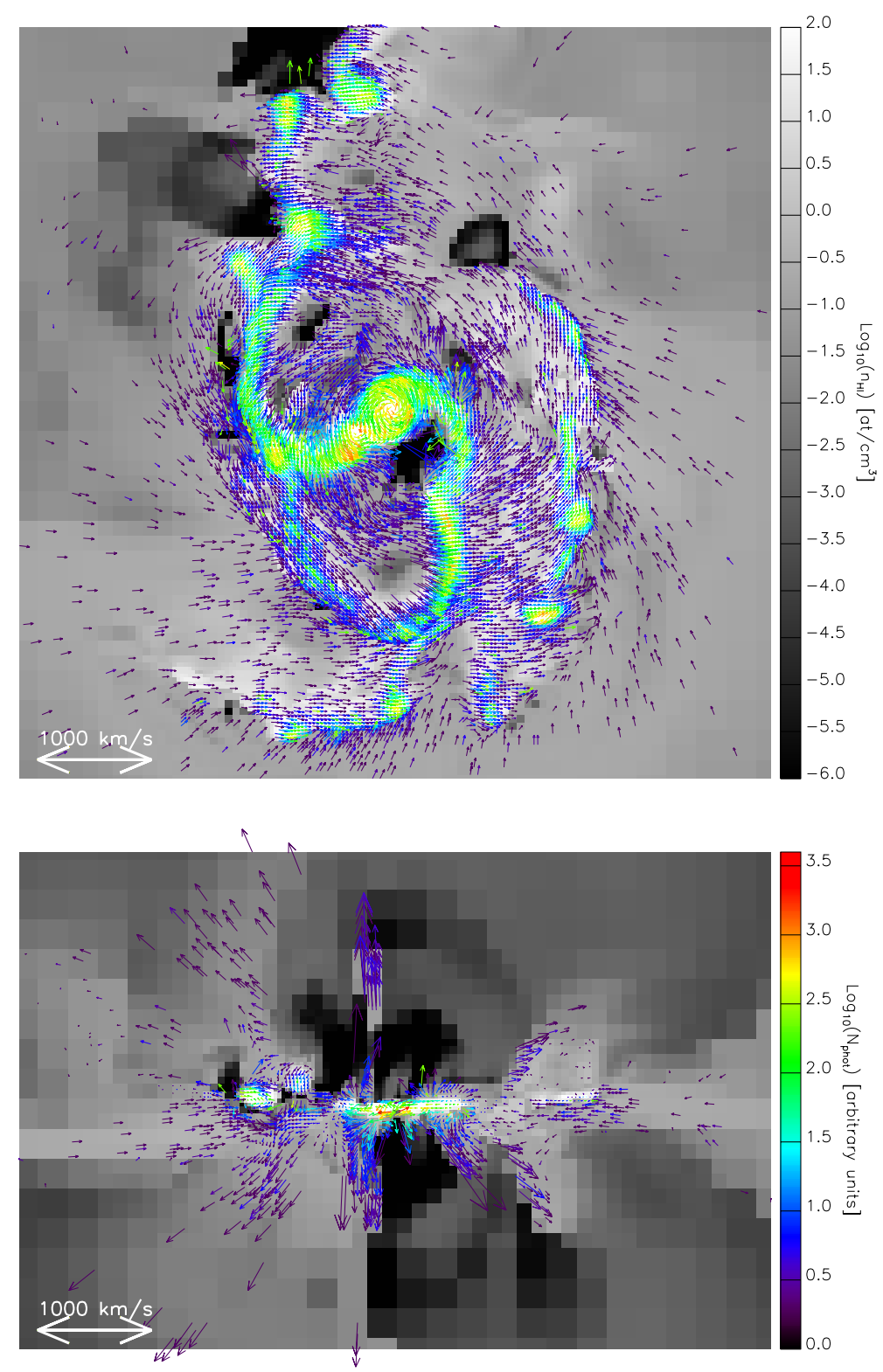

Figure 3.13: The simulated galaxy G2 from Verhamme et al. 2012. Shown is a slice in density (grey contours), the projected number of photon escaping from a region (colored contours) and the velocity field (arrows; scale in the lower left shows amplitude). Reprinted with permission (c) ESO (Figure 7 in Verhamme et al. 2012) 


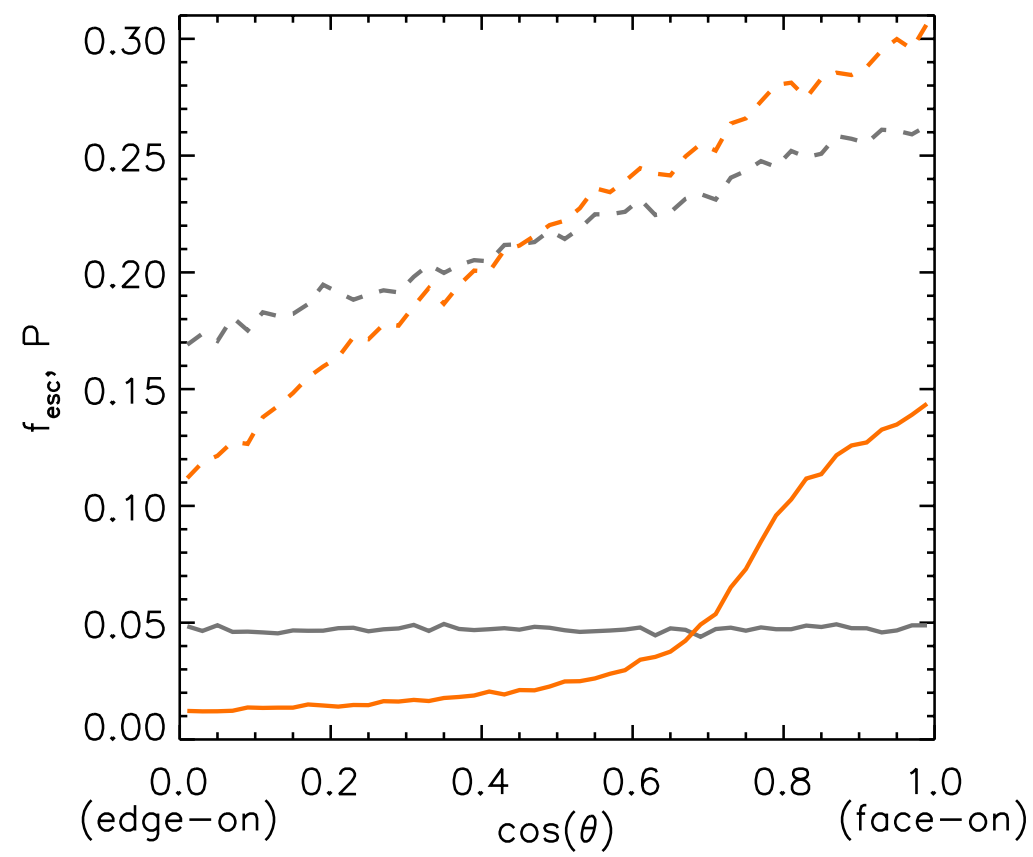

Figure 3.14: Escape fraction $f_{e s c}$ as a function of inclination of the escape direction (red) and probability $P$ for a photon to escape if it has an initial inclination $\cos \theta$ (black), shown for continuum (dashed) and Lyman- $\alpha$ photons (solid) as obtained by Verhamme et al. 2012. Reprinted with permission (c) ESO (Figure 8 in Verhamme et al. 2012)

emission direction which is a consequence of resonant scattering: Since photons change direction many times before escape/absorption, their initial direction does not matter. The dashed black/red lines show the same data for the continuum photons.

Here, having an initial emission direction close to face-on favors escape by a factor of 2 over edge-on directions (black dashed line) because continuum photons are rarely scattered on hydrogen compared to Lyman- $\alpha$ radiation. Also, the escape towards face-on directions is favored, but only by a factor of 3 in the case of the continuum photons (red dashed line). This is again a consequence of the continuum not being subject to resonant scattering. Comparing the two species, we see that the observed EW changes depending on the direction of observation because of this difference in directional boosting for Lyman- $\alpha$ /continuum: The ratio of observed Lyman- $\alpha$ photons and observed continuum photons per wavelength is larger in face-on directions. As figure 3.15 shows, this is indeed the case. Shown is the EW observed along random lines of sight. The $x$-axis shows the absolute value of the inclination for the corresponding line of sight. While the EW becomes negative 
edge-on (indicating absorption and not emission in the Lyman- $\alpha$ line), it rises to $100 \AA$ for face-on directions. The spectra from their simulation show differences with respect to the line of sight as well: While it is double-peaked observed edge-on with a slightly enhanced red peak due to outflowing gas, it shows a single red peak for face-on directions.

The strong inclination dependency of Lyman- $\alpha$ radiation in this disk galaxy simulation is a result that implies a possible bias in large-scale surveys as described in 2.2. Another result is that the Neufeld scenario fails to enhance the Lyman- $\alpha$ escape, since Lyman- $\alpha$ photons are primarily produced in the dense, dusty clouds (in which young stars typically live) where they are absorbed very efficiently. This is the reason that the observed EW never reaches or exceeds the intrinsic EW - in terms of the boosting factor $b$ from Gronke \& Dijkstra 2014, they reach a maximum of $b \sim 0.5$ despite the large directional dependency.

Besides Verhamme et al. 2012, there is no study of the inclination effect in an isolated disk galaxy simulation except for Behrens \& Braun 2014 (see section 4.3 for a reproduction). In this work, we investigated the Lyman- $\alpha$ transfer in a simulation originally presented in Braun et al. 2014. The galaxy simulation includes a complex model for the interplay between a multiphase ISM, the star formation, feedback,

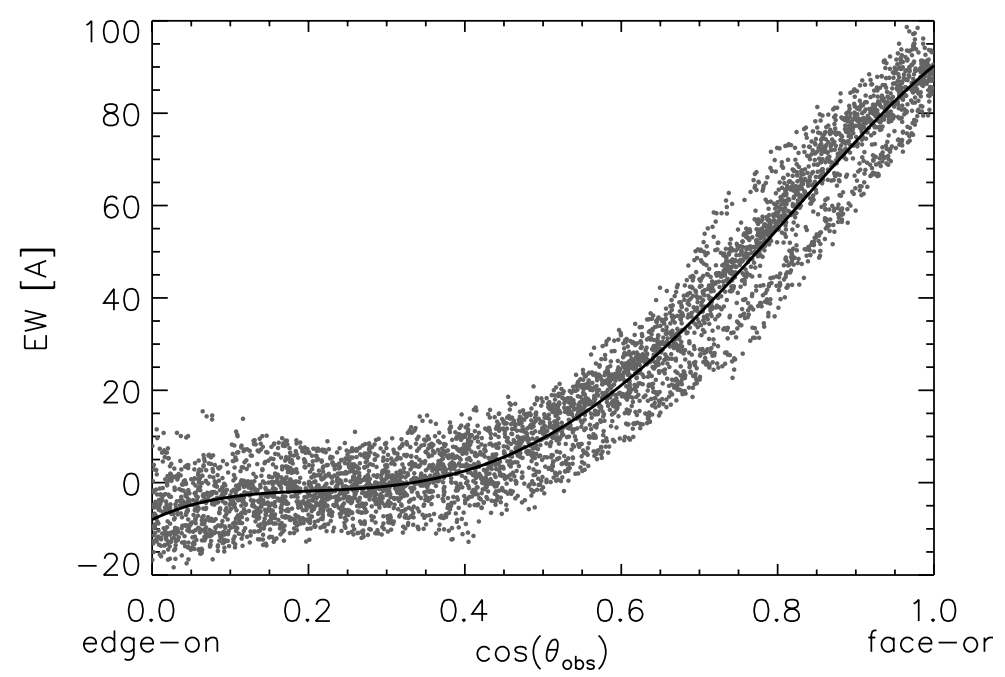

Figure 3.15: EWs as observed along random lines of sight for the simulation in Verhamme et al. 2012, shown as a function of the inclination $\cos \Theta$. The line shows a polynomial fit to the distribution. Reprinted with permission (c) ESO (Figure 10 in Verhamme et al. 2012) 
and the turbulent energy, called MIST ${ }^{1}$. While Verhamme et al. 2012 assume a constant contribution of turbulent motions to the Doppler frequency (see section 1.3), in turn influencing the cross section of the photons in neutral hydrogen, we can use the subgrid scale turbulent energy assigned to each cell in the simulation domain (see Schmidt \& Federrath 2011). Star formation is regulated self-consistently by the feedback, including both thermal and turbulent feedback, yielding a star formation rate following a Schmidt law (i.e. the star formation rate is a function of the gas density). The resolution of the simulation is $\sim 30$ pc. It starts from a marginally stable gaseous disk with a gas mass of $10^{10} \mathrm{M}_{\odot}$, and evolves into a clumpy disk with star-forming regions being formed and successively disrupted by feedback. We performed calculations of the radiative transfer on snapshots 1, 1.5, and 2 Gyr after initialization. In our setup, we closely follow Verhamme et al. 2012 to ensure comparability. However, the simulated disk galaxy is morphologically different from theirs which is a result of the different dynamics included in the simulation. In particular, their disk consists of few massive clumps, with large underdense regions between them, while our disk features small clumps connected by a large number of small, transient spiral arms. A visualization of the disk galaxy can be seen in figure 1 (section 4.3). The first column shows the projected neutral hydrogen density for the different snapshots, the second shows the dust distribution, and the third one shows the local Lyman- $\alpha$ luminosity for the three snapshots. In contrast to Verhamme et al. 2012, we launch both Lyman- $\alpha$ and continuum photons according to the local Lyman continuum photon density that is calculated by the feedback module. Nevertheless, this method also traces the recent local star formation. Lyman- $\alpha$ photons are emitted in frequency space according to a Gaussian with a width of $10 \mathrm{~km} / \mathrm{s}$. Continuum photons are emitted with a flat spectrum in the range of $\pm 2 \times 10^{4} \mathrm{~km} / \mathrm{s}$, the intrinsic EW is set to $200 \AA$. The set of simulations we call the 'fiducial' one uses the dust model of Verhamme et al. 2012 but with a reduced dust content (lowered by a factor of 25) for ease of interpretation. We also ran a so-called 'realistic' set, using the original dust model by Verhamme et al. 2012. Our main conclusions are the same for both sets.

We can confirm the existence of a strong anisotropy in Lyman- $\alpha$ properties as found by Verhamme et al. 2012. There are, however, some discrepancies with their results. Our escape fraction in the fiducial case is higher for both Lyman- $\alpha$ photons

1 Multi-phase Interstellar medium, Star formation, and Turbulence 
(14 to $20 \%$ vs. $5 \%$ ) and continuum photons (about $80 \%$ vs. $22 \%$ ) due to the fact that we reduced the dust content artificially: In the realistic case, we find $0.2-14 \%$ for the Lyman- $\alpha$ photons. Additionally, we find a top-bottom asymmetry for the Lyman- $\alpha$ properties: It is easier for photons to escape towards the top $(+z$-axis) than to the bottom (-z-axis). We suggest this to be a result of an asymmetry in the outflows that arise from the supernova feedback.

More importantly, we find a strong variation of the Lyman- $\alpha$ properties from snapshot to snapshot. This can be seen in figure 3 (section 4.3). It is the same type of plot as figure 3.14 from Verhamme et al. 2012, but for the three snapshots from left to right. The solid, red line shows the distribution of escape directions. The slope and amplitude change strongly from snapshot to snapshot. This results in variations of the observed EW distribution, shown in figure 4 for the fiducial case (section 4.3). Similar to figure 3.15, the plot shows the EW observed by a set of randomly chosen lines of sight, with blue symbols corresponding to the 1 Gyr snapshot, red symbols for the 1.5 Gyr snapshot, and the data for the 2 Gyr snapshot in green. Different from figure 3.15, we show the data as a function of the inclination $\cos \Theta$ and not as a function of $|\cos \Theta|$ to account for the top-bottom asymmetry. The maximum and average values for the observed EW change with snapshot to snapshot. In particular, the observed maximum EW in approximately face-on directions rise from 50 to about $225 \AA$ from 1 Gyr to 2 Gyr (also note the top-bottom asymmetry for 1.5 and 1 Gyr here) for the fiducial runs. Analysis reveals that the origin of these differences in time are small $(<500 \mathrm{pc})$ cavities in the gas distribution, carved out by supernova explosions. We show some examples of these cavities in figure 6 in section 4.3 (lower panel). The black contours indicate the Lyman- $\alpha$ emissivity, the colored contours indicate density. In these regions, the stellar feedback has created cavities with lines of sight that have low optical depth. The lines of sight are typically orthogonal to the plane of the disk, therefore boosting face-on escape. About $50 \%$ of all transmitted Lyman- $\alpha$ photons escape from these cavities in the 1.5 and 2 Gyr snapshot. The pronounced top-bottom asymmetry in the 1.5 Gyr is therefore explained by the fact that the transmitting cavities in this snapshot feature cavities letting photons dominantly pass towards the $+z$-axis, while the 2 Gyr run exhibits cavities transmitting to the top and to the bottom, therefore reducing the asymmetry. The 1 Gyr snapshot does not feature large transmitting bubbles, and therefore has low EWs in general and in especially relatively low EWs in face-on directions. One important property of the actively transmitting cavities is that they still inhabit 
very recent star formation. This is only possible for a very short period in time in small star-forming regions, since the feedback will also cut off gas supply for star formation. For large stellar clusters in the simulation, the dynamics are dominated by the gravitational potential of the stars. In this case, we expect the star-forming region not to be completely disrupted by the feedback. Instead, we expect it to cyclically switch between states of low and high star formation and therefore weak and strong Lyman- $\alpha$ emission. Our conclusion is that this points towards a temporal variability of LAEs on the time scale of the star formation.

Apart from these findings, we also tested the influence of various physical parameters on the radiative transport. As expected, increasing dust or neutral hydrogen mass reduces escape fractions and EWs. We also studied a dust-free case. Here, we find the EW to be boosted up to $450 \AA$ for face-on directions, while edge-on observers see Lyman- $\alpha$ in absorption. 



\section{CHAPTER 4}

Accepted Publications

4.1 Effects of Lyman-Alpha Scattering in the IGM on Clustering Statistics of Lyman-Alpha Emitters 


\title{
Effects of Lyman-alpha scattering in the IGM on clustering statistics of Lyman-alpha emitters ${ }^{\star}$
}

\author{
C. Behrens and J. Niemeyer \\ Institut für Astrophysik, Georg-August Universität Göttingen, Friedrich-Hundt-Platz 1, 37077 Göttingen, Germany \\ e-mail: [cbehren;niemeyer] @astro.physik.uni-goettingen.de \\ Received 26 January 2013 / Accepted 3 June 2013 \\ ABSTRACT \\ We investigate the correlations between the observed fraction of Lyman-alpha (Ly $\alpha$ ) emission from star-forming galaxies and the \\ large-scale structure by post-processing snapshots of a large, high-resolution hydrodynamical simulation with a Ly $\alpha$ radiative transfer \\ code at redshifts $z=4,3,2$. We find correlations of the observed fraction with density, density gradient along the line of sight, \\ velocity, and velocity gradient along the line of sight, all within the same order of magnitude (tens of percent). Additionally, a \\ correlation with the angular momentum of the dark matter halo is detected. In contrast to a previous study, we find no significant \\ deformation of the two-point correlation function due to selection effects from radiative transfer in the intergalactic medium within \\ the limited statistics of the simulation volume.
}

Key words. large-scale structure of Universe - radiative transfer - galaxies: high-redshift - intergalactic medium

\section{Introduction}

Galaxies with strong Lyman-alpha ( $\operatorname{Ly} \alpha)$ emission features, socalled Ly $\alpha$ emitters (LAEs), are powerful probes of galaxy evolution and cosmological large-scale structure. There are indications that LAEs, or some subset thereof, evolved into today's Milky Way type galaxies (Guaita et al. 2010), hence their properties may shed light on our own Galaxy's youth. Also, future observations might be able to detect the first galaxies via $\operatorname{Ly} \alpha$ emission (e.g., Dijkstra \& Wyithe 2010).

Ly $\alpha$ emitters can be detected very efficiently in narrowband or integral-field spectrographic surveys such as the Hobby-Eberly Telescope Dark Energy Experiment (HETDEX; Adams et al. 2011). It has been proposed that they act as tracers of the underlying matter distribution at higher redshifts than currently accessible for galaxy redshift surveys. Specifically, HETDEX aims to use the power spectrum of $\sim 800000$ LAEs to measure the Hubble parameter $H(z)$ and angular distance $D_{\mathrm{A}}(z)$ at redshifts between $z=1.9$ and 3.5 with percent-level accuracy in order to constrain the early dynamics of dark energy.

Every interpretation of LAE observations needs to take into account the resonant nature of $\operatorname{Ly} \alpha$ scattering (e.g., Cantalupo et al. 2005; Dijkstra et al. 2006; Adams et al. 2009; Zheng et al. 2010; Yajima et al. 2012; Schaerer et al. 2011; Hansen \& Peng Oh 2006). The large cross section of Ly $\alpha$ photons scattering with neutral hydrogen (HI) strongly correlates the observed Ly $\alpha$ spectra and apparent luminosities along any given line of sight with the density and velocity structure of the intervening HI (Dijkstra et al. 2006; Laursen et al. 2011). As a result, large amounts of information are encoded in the observations. On scales of the emitting galaxies and their circumgalactic material, Ly $\alpha$ spectra are very sensitive to the presence of clumps, dust, and in- or outflows (Zheng \& Miralda-Escude 2002; Dijkstra et al. 2006; Laursen et al. 2009b; Schaerer et al. 2011; Barnes et al. 2011).

\footnotetext{
$\star$ Appendices are available in electronic form at http://www. aanda.org
}

Recent simulations also highlight the possibility of a strong inclination dependence of the $\operatorname{Ly} \alpha$ observed fraction which, in turn, depends on the morphology of the gaseous disk (Laursen \& Sommer-Larsen 2007; Yajima et al. 2012; Verhamme et al. 2012). Mapping the theoretical predictions, mostly from numerical simulations, to properties of observed LAEs has only just begun (e.g., Nagamine et al. 2010; Shimizu et al. 2011; Forero-Romero et al. 2011; Dayal \& Ferrara 2012) and promises to be a rich field of research in the coming years.

On the other hand, correlations of apparent LAE luminosities with the matter distribution induced by Ly $\alpha$ radiation transport (RT) effects can also contaminate the clustering statistics of LAEs on larger scales. If they reach out to scales relevant for the extraction of cosmological parameters, they need to be accounted for by corrections in the LAE power spectrum in real and redshift space. This effect was demonstrated by Zheng et al. (2011a, hereafter ZCTM11) using a Monte-Carlo Ly $\alpha$ RT calculation on the background of a cosmological simulation snapshot at $z=5.7$. Details of their setup and further investigations of the luminosity, spectra and observed fractions of LAEs in their simulation can be found in Zheng et al. (2010, hereafter ZCTM10) (see also Zheng et al. 2011b for details on extended LAE halos). They found significant correlations of the Ly $\alpha$ observed fraction, i.e. the fraction of photons that are not scattered out of the line-of-sight during their passage through the intergalactic medium (IGM), with the smoothed IGM density and velocity fields. By far the biggest effect was seen in correlations with the velocity gradient field, accompanied by a strongly anisotropic signature in the two-point correlation function for LAEs in redshift space. If present also at lower redshifts, an effect of this magnitude would seriously affect the interpretation of LAE large-scale structure surveys like HETDEX. This was investigated in more detail in Wyithe \& Dijkstra (2011) by means of analytic and numerical models for LAE spectra with in- and outflows, which the authors used to calibrate a modified parametrization for the LAE power spectrum. Using an 
A\&A 556, A5 (2013)

Alcock-Paczynski test, they then showed that the accuracy of HETDEX measurements could potentially be seriously compromised by Ly $\alpha$ RT effects. In Greig et al. (2013), this analysis was extended to include the LAE bispectrum which allows the degeneracy betweeen the Ly $\alpha$ RT effects and the gravitational redshift-space distortion that is present at the level of the power spectrum alone to be broken.

Although they were most extensive numerical investigation of Ly $\alpha$ RT on cosmological scales, the methodology and resolution of the simulation analyzed by ZCTM10/11 were inadequate to capture the nonlinear hydrodynamics in the circumgalactic medium (CGM) surrounding LAEs. Instead of a full hydrodynamical simulation, ZCTM10/11 employed a hybrid scheme which assumed hydrostatic equilibrium for the gas in virialized halos. Consequently, no outflows were present in their simulation, and the infall was purely gravitational with no hydrodynamical modifications on CGM scales. In Wyithe \& Dijkstra (2011), galactic outflows were modeled in a simplified way that was also assumed to be independent of the environment on linear scales. The exact degree to which nonlinear flows on scales $\$ 100 \mathrm{kpc}$ are correlated with their large-scale environment is still unclear, but can plausibly be assumed to be non-vanishing. In this case, the well-known strong sensitivity of LAE properties on CGM/IGM flows (e.g., Dijkstra et al. 2007; Iliev et al. 2008; Laursen et al. 2011) will be reflected to some extent in the largescale statistics. Including the effects of fully hydrodynamical inand outflows was one of the main motivations for this work.

Another question raised by ZCTM10/11 is the redshift dependence of the observed correlations. This is particularly important for HETDEX which will cover a redshift range which is significantly below the one explored by ZCTM10/11.

In this work, we revisit the analysis of ZCTM10/11 using Ly $\alpha$ RT on the background of snapshots of the MareNostrum-Horizon simulation (Ocvirk et al. 2008) at redshifts of $z=2,3$, and 4. The MareNostrum simulation has a spatial resolution of $1 \mathrm{kpc}$ (physical) and includes a model for supernova feedback driving galactic outflows in a self-consistent fashion. In addition to evaluating the correlations of the $\operatorname{Ly} \alpha$ observed fraction with the IGM density and velocity on linear scales, we tested for a possible dependence on the orientation of the halos' angular momentum relative to the line of sight, serving as a proxy for the orientation of the galactic disk. We find a positive result, indicating that tidal alignment of halos might give rise to additional spurious signals in redshift space distortions (Hirata 2009).

Our numerical techniques for Ly $\alpha$ RT are summarized in Appendix B. We describe the details of the simulation and our postprocessing runs in Sect. 2. Our results for the correlations of large-scale density and velocity fields with Ly $\alpha$ observed fractions are presented in Sect. 4.

\section{Ly $\alpha$ radiation transport calculations and analysis}

\subsection{The horizon-MareNostrum galaxy formation simulation}

We applied our radiative transfer code LyS (see the Appendix for details) to snapshots taken from the Horizon-MareNostrum Galaxy Formation run which was presented and described in Ocvirk et al. (2008). The simulation was run using an updated version of the adaptive-mesh refinement (AMR) code Ramses (Teyssier 2002), including metal dependent cooling, star formation, a simple supernova feedback model and UV heating. The box had a comoving size of $50 \mathrm{Mpc} / \mathrm{h}$ with a physical resolution of $1 \mathrm{kpc}$. Star formation took place in the interstellar medium (ISM), defined as gas with a number density greater than $0.1 n_{\mathrm{H}} / \mathrm{cm}^{3}$. The dark matter particle mass was $8 \times 10^{6} M_{\odot}$ with a total particle count of $1024^{3}$. The simulation was run assuming a standard $\Lambda \mathrm{CDM}$ cosmology with $\Omega_{\mathrm{M}}=0.3, \Omega_{\Lambda}=0.7$, $\Omega_{\mathrm{B}}=0.045, H_{0}=70 \mathrm{~km} \mathrm{~s}^{-1} / \mathrm{Mpc}$ and $\sigma_{8}=0.9$. For more information on the spectroscopic properties of galaxies in the simulation, see Gay et al. (2009).

\subsection{Preprocessing}

We rebuilt the AMR hierarchy of the MareNostrum run and calculated the temperatures of the gas cells from the specific pressure assuming photoionization equilibrium (Katz et al. 1996). In the ISM regions, the breakdown of single component fluid description leads to artificially high temperatures. To overcome this problem, we enforce an upper limit of $2.5 \times 10^{4} \mathrm{~K}$ on the ISM temperature.

In order to find the emission spots for the $\operatorname{Ly} \alpha$ photons, we used the HOP algorithm (Eisenstein \& Hut 1998) to produce a halo list. We used a standard set of parameters $\left(\delta_{\text {outer }}=\right.$ $\left.80, \delta_{\text {saddle }}=200, \delta_{\text {peak }}=240\right)$ and rejected particle groups that consist of less than 600 particles after the regrouping process, corresponding to a cut-off mass of $4.8 \times 10^{9} M_{\odot}$. With this cutoff, we have a sample size of $\sim 42000 / 49000 / 51000$ emitters at redshift $4 / 3 / 2$. The mass range of these emitters is $5 \times 10^{9} M_{\odot}$ to $3.1 \times 10^{12} / 8.0 \times 10^{12} / 3.3 \times 10^{13} M_{\odot}$ for $z=4 / 3 / 2$.

\section{Our simulations and analysis}

The Ly $\alpha$ RT was run as a postprocessing step on simulation snapshots at redshift $z=2,3$ and 4 . In addition to the fiducial case, we re-ran our simulation at redshift 4 with a) the Hubble flow; b) the peculiar velocity field; and c) both turned off for interpretation and comparison. To achieve this, we set the Hubble constant in Eq. (B.1) to zero and/or set the total bulk velocity $v$ in Eq. (A.2) to zero so that the restframe of each gas cell is identical to the restframe of the emitter. The thermal motion of the gas is, however, not affected by this procedure. We also ran the redshift 4 snapshot along three different lines of sight. For details on the initialization of spectra and luminosities, we refer the reader to Appendix B. The spatial resolution of our output array is $16.3 \mathrm{kpc} / \mathrm{h}$ (comoving), corresponding to $0.67 / 0.74 / 0.91^{\prime \prime}$ at redshift $4 / 3 / 2$

The output matrix was converted into physical fluxes and surface brightnesses. By integrating over the spectral information of the output matrix, we obtained surface brightnesses of each $\left(i_{y}, i_{z}\right)$-pixel. For each halo, we ran a friend-of-friend algorithm to find the apparent luminosity of the source. If the pixel covering the central position of the halo had a surface brightness exceeding a threshold $\eta$, we added its flux to the flux of the source, and connected adjacent pixels that are above the threshold.

Using the total flux of each source obtained with this procedure, we defined the source's inferred apparent luminosity $L_{\text {apparent }}$. As a result, we can compute the fraction

$\epsilon=\frac{L_{\text {apparent }}}{L_{\text {intrinsic }}}$

of the intrinsic luminosity that was detected. Since this quantity measures the part of the intrinsic luminosity that an observer would see, it plays the role of an observed fraction, and hereafter we will refer to it by this term. We note that in our case, the difference between intrinsic and inferred luminosity is not due 
C. Behrens and J. Niemeyer: Effects of Lyman-alpha scattering in the IGM on clustering statistics of Lyman-alpha emitters

to destruction of photons by dust, but due to the application of a detection limit.

To prevent source blending, we identified and ignored sources that would swallow up other emitters during the postprocessing, although these blended source are quite rare $(\sim 5 \%$ of the total number) and did not affect our results very much.

The chosen value of the surface brightness limit $\eta$ is arbitrary in the sense that we did not include dust and did not model the systematic errors of a real observation in detail. We used a similiar value to the one used by ZCTM10,

$\eta=5 \times 10^{-19} \mathrm{erg} \mathrm{s}^{-1} \mathrm{~cm}^{-2} \operatorname{arcsec}^{-2}$.

This particular value was chosen to be well above the noise level in the output data. We note that this threshold is orders of magnitude smaller than the detection threshold of HETDEX, for example, which is $\eta \simeq 10^{-17} \mathrm{erg} \mathrm{s}^{-1} \mathrm{~cm}^{-2} \operatorname{arcsec}^{-2}$. We chose a lower value to be comparable with ZCTM10 but also not to degrade statistics by having only a few sources detected.

The observed fraction of $\operatorname{Ly} \alpha$ along a specific line of sight is related to the density and velocity structure along the line of sight (Wyithe \& Dijkstra 2011); note that ZCTM10 and ZCTM11 stress the importance of the structure in the perpendicular directions. In order to find the correlations between the dark matter distribution in the MareNostrum simulation and the observed fractions on linear scales relevant for LAE redshift surveys, we closely followed the strategy described by ZCTM10. The dark matter particles were interpolated onto a grid using a cloud-incell algorithm and smoothed out on a scale of 10/12/15 Mpc/h with a top-hat filter of this diameter. We chose this filtering scale to obtain the density field in the linear regime at redshift $4 / 3 / 2$ consistent with our calculations below. From the smoothed density field, we calculated the linear velocity field and density/velocity gradients along the line of sight.

The smoothed density and velocity fields are well described by linear theory. From the continuity equation

$\dot{\delta}=-\frac{1}{a} \operatorname{div} \boldsymbol{u}$

one finds the peculiar velocity field $\boldsymbol{u}_{k}$ in Fourier space:

$\boldsymbol{u}_{k}=f H a \frac{\mathrm{i} \boldsymbol{k}}{k^{2}} \delta_{k}$.

We are only interested in the line-of-sight component of the velocity field which we assume to be parallel to the $x$-axis here

$u_{x}=f H a \sum_{k} \frac{k_{x} \mathrm{i}}{k^{2}} \delta_{k} \mathrm{e}^{\mathrm{i} k \cdot r}$.

The spatial derivative of the velocity field in the line of sight is given by

$\frac{\partial u_{x}}{\partial x}=-f H a \sum_{k} \frac{k_{x}^{2}}{k^{2}} \delta_{k} \mathrm{e}^{\mathrm{i} k \cdot r}$

We also calculate the angular momentum of the individual halos directly from the particle data.

\section{Results}

\subsection{Overview}

Figure 1 shows a spectrally integrated image of the LAEs in the box as seen by an observer located along the positive $z$-axis. In Fig. 2, the spatially integrated spectrum of an emitter with

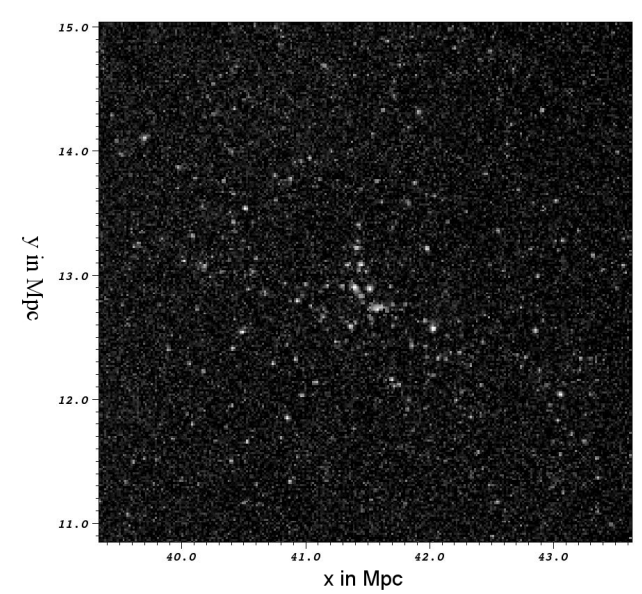

Fig. 1. Lyman- $\alpha$ image of a small part $(\sim 5 \times 5 \mathrm{Mpc})$ of the simulation volume at redshift 4 . The observer is located along the positive $z$-axis, the snapshot corresponds to $z=4$.

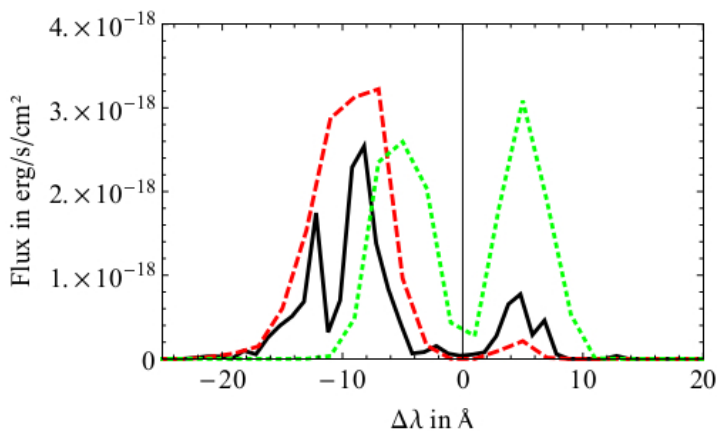

Fig. 2. Typical Spectrum of an emitter with a mass of $1.9 \times 10^{10} M_{\odot}$. Shown is the spectrum for the fiducial simulation (solid line), the simulation without any peculiar velocities and Hubble flow (dotted line), and a simulation where peculiar velocities were enabled but the Hubble flow was switched off (dashed line). Wavelength is given with respect to the observer's restframe. For the fiducial simulation, the redshift due to the Hubble flow within the simulation box was ignored.

a mass of $1.9 \times 10^{10} M_{\odot}$ is shown for three different setups: the solid line shows the spectrum of the fiducial run, the dotted line shows a run with all peculiar motions and the Hubble flow artificially set to zero, and the dashed line is obtained from a simulation where only the Hubble flow was switched off. For the case without peculiar motions and Hubble flow, we clearly see the typical double-peaked spectrum that one would get from a static sphere (see Fig. B. $2^{1}$ ). Deviations from this solution result from anisotropic density fields. Turning on the velocity field, we obtain the typical spectrum of an infalling sphere (see Fig. B.3) which is intuitive since the region in the halo's vicinity should show clear infall. Photons are thereby shifted to the blue side of the spectrum, undergoing only a few scatterings after leaving the halo. If we switch on the Hubble flow, the situation changes. Blue photons leaving the ISM are shifted back into the line center and scattered in the intervening IGM. As a consequence, the observed flux is significantly reduced because photons are scattered out of the line of sight. We note again that these photons

We note that in Fig. 2, wavelength is shown instead of frequency. 


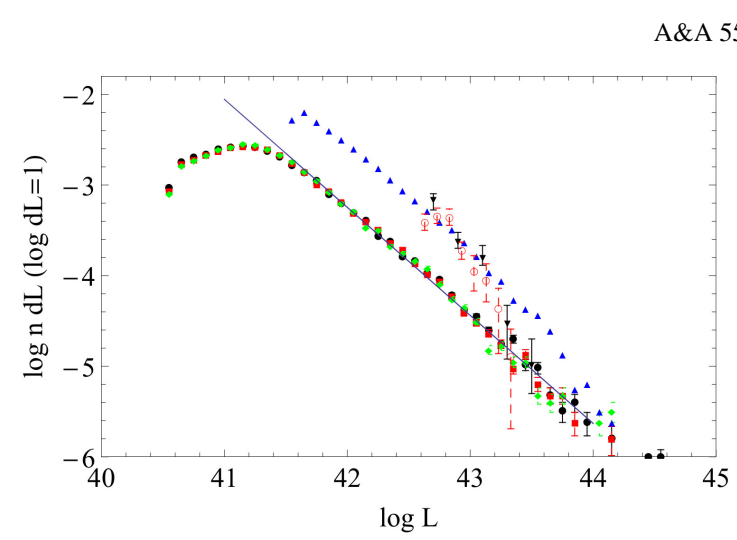

Fig. 3. Luminosity function from our simulation at $z=4$ for three different lines of sight. The observer is set along the $x$-axis (circles), $y$-axis (boxes), and $z$-axis (diamonds), respectively. Triangles without error bars show the distribution of the intrinsic luminosity, hollow circles the observed luminosity function for $z=4.5$ (Wang et al. 2009), and the flipped triangles the luminosity function for $z=3.7$ as observed by Ouchi et al. (2008). The line depicts the log-linear fit to the data, yielding a power law with $\alpha=-1.19$. Number density is in units of $\mathrm{Mpc}^{-3} / \log (L)$.

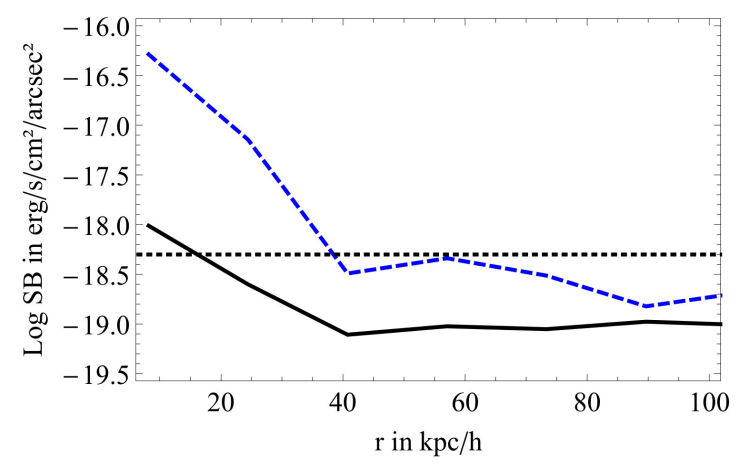

Fig. 4. Surface brightness profiles for two sources with observed fractions of $\sim 30 \%$, with masses of $1.9 \times 10^{10} M_{\odot}$ (black solid line) and $7 \times 10^{11} M_{\odot}$ (blue dashed line). The horizontal dashed line indicates the detection threshold. On average, there were only 2 pixels per source above the detection threshold, and over $90 \%$ are detected in less than 5.5 pixels.

are not destroyed by dust, but they contribute to a noise level of diffuse emission. The Hubble flow transports photons from the blue to the red side of the spectrum. Once photons have left the line center to the red side they will be further redshifted, making subsequent scatterings more and more improbable.

The surface brightness profiles for two sources are shown in Fig. 4. It is worth noting that while ZCTM10 reported extended Ly $\alpha$ halos $(r \sim 300 \mathrm{kpc})$ with surface brightnesses of $\sim 10^{-20} \mathrm{erg} \mathrm{s}^{-1} \mathrm{~cm}^{-2} \operatorname{arcsec}^{-2}$ at $z=5.7$ we find more compact sources. These differences might be partly attributed to the lower redshift in our simulation. Additionally, as we resolve the ISM at least marginally, most of the scatterings happen in the ISM where the optical depth is high because of high HI densities. This shifts the photons out of resonance and reduces the optical depth of the immediate surroundings of the halo. Since later IGM scatterings happen far away from the emitting halo, they contribute to a diffuse background rather than to an extended Ly $\alpha$ halo.
One can also obtain the luminosity function for our simulation, shown in Fig. 3 for the three different lines of sight at redshift 4 . We also show the intrinsic luminosity function (triangles) of our simulation. The overall shape of the luminosity function is not changed by the RT process. The drop at the low luminosity is due to incompleteness, since LAEs that are below the detection limit have an apparent luminosity of zero. The detection threshold and the assigned intrinsic luminosity introduce a free parameter in our model, shifting the luminosity function by a constant factor. Since we are mostly interested in changes of the observed fraction relative to the mean, we ignore this shift here.

\subsection{Correlations between large-scale structure and observed fraction}

In this section we focus on our results from the redshift 4 snapshot.

Figure 5 shows how the observed fractions correlate with the dark matter overdensity $\delta$, the density gradient along the line of sight, the line-of-sight velocity and the line-of-sight velocity gradient, all evaluated at the positions of the sources for three different lines of sight. The observed fraction is given relative to the mean observed fraction

$\Delta \epsilon=\frac{\epsilon}{\bar{\epsilon}}$

The mean observed fraction $\bar{\epsilon}$ is $30 / 63 / 87 \%$ for our fiducial runs at redshift 4/3/2. ZCTM10 find a lower mean observed fraction of a few percentage points at redshift 5.7. Laursen et al. (2011) also calculated observed fractions from nine simulated galaxies at 3.5. Although their sample is small, their mean observed fraction is around $24 \%$ (we also note they do include photon destruction by dust).

We use the full sample of emitters and generate the plots of the correlations applying a moving average to the data set, averaging over 4000 emitters per data point. As described above, densities and (linear) velocities are obtained from the smoothed dark matter particle data. Density gradient, velocity, and velocity gradient plots depend on the line of sight chosen, so we plot the relevant component $x_{i}$ or the derivative with respect to $x_{i}$, where $x_{i}=x, y, z$ for observers located along the respective axis.

The correlation between density and velocity gradient follows directly from the continuity equation (see Eq. (3)) Statistically, this also holds for the individual components of the divergence, i.e., the line-of-sight velocity gradient. The correlation between line-of-sight velocity and line-of-sight density gradient is quite intuitive: halos beyond a large-scale overdensity move towards it and hence towards the observer, and vice versa. Both correlations are shown in Figs. 10 and 11. Because of this direct connection between the two pairs of observables, we discuss each of the pairs together.

As can be seen in Fig. 5, correlations differ between different lines of sight. Although the trends are mutually consistent, deviations of up to $10 \%$ are clearly visible. We interpret this as a consequence of cosmic variance. Investigation indeed shows that the halo distribution and velocity fields clearly differ among different lines of sight, which can be expected for a box of this size. As an example, in Fig. 8, the $x / y / z$ component of the halos' velocity is plotted against the $x / y / z$ coordinate. For this plot, velocities are directly obtained from the dark matter particles, but the linear approximation we use to build the correlation plots shows the same behavior, i.e., a large-scale, sine-like signal. This 
C. Behrens and J. Niemeyer: Effects of Lyman-alpha scattering in the IGM on clustering statistics of Lyman-alpha emitters
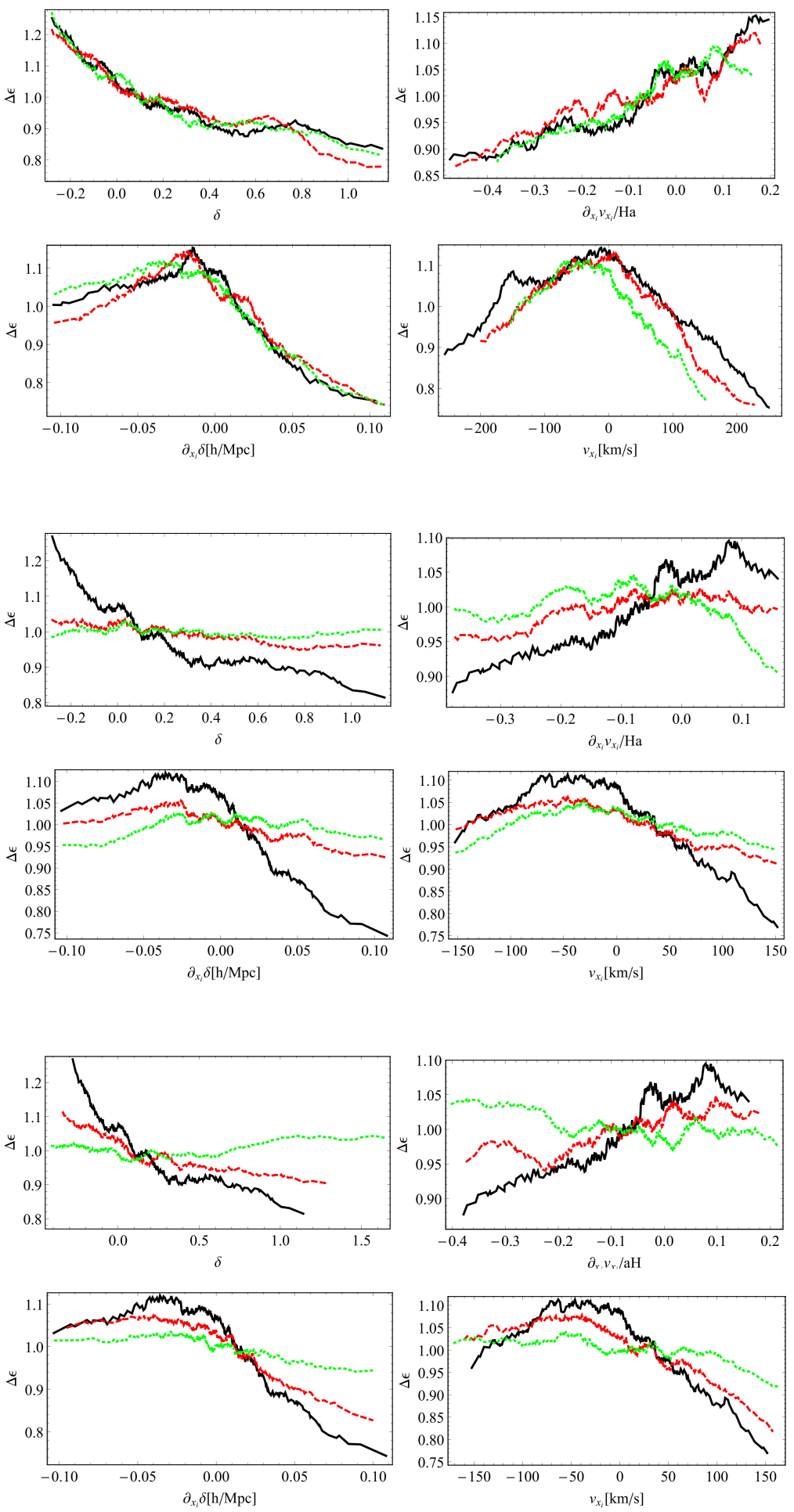

Fig. 5. Correlations between observed fraction and large-scale structure. On the $y$-axis, the relative observed fraction is shown in all four plots. The $x$-axis shows density (upper left), the-line-of-sight velocity gradient (upper right), the line-of-sight density gradient (bottom left) and the line-of-sight velocity (bottom right). The three different lines correspond to observer's location along the $x$-axis (black solid), $y$-axis (red dashed), and $z$-axis (green dotted).

Fig. 6. Same as Fig. 5, but here the lines correspond to the fiducial case (black solid), a run without peculiar motions and Hubble flow (red dashed), and a simulation with peculiar velocities but without Hubble flow (green dotted) as seen by an observer along the $z$-axis.

Fig. 7. Same as Fig. 5, showing the redshift dependence of the correlations. The data for a redshift of $z=4$ (black solid), $z=3$ (red dashed), and $z=2$ (green dotted) is shown. The observer is located along the $z$-axis. 
A\&A 556, A5 (2013)

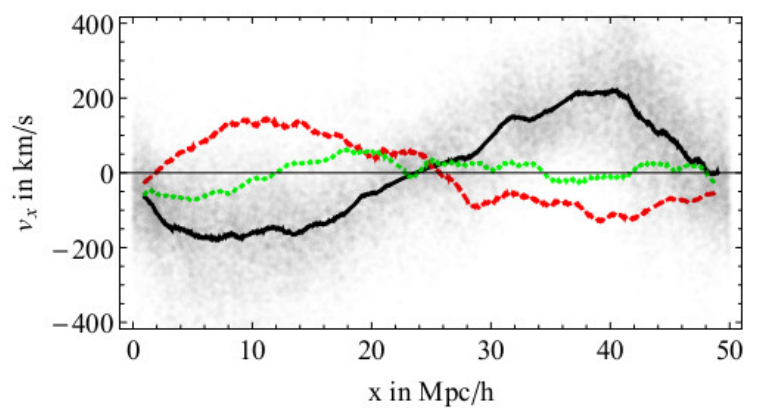

Fig. 8. Averaged line-of-sight component of the halo velocities versus their location along the line of sight at $z=4$ for three lines of sight parallel to the $x$-/y-/z-axis (black solid/red dashed/green dotted). To illustrate the scatter, the distribution of halos is plotted in gray for the $x$-axis data.

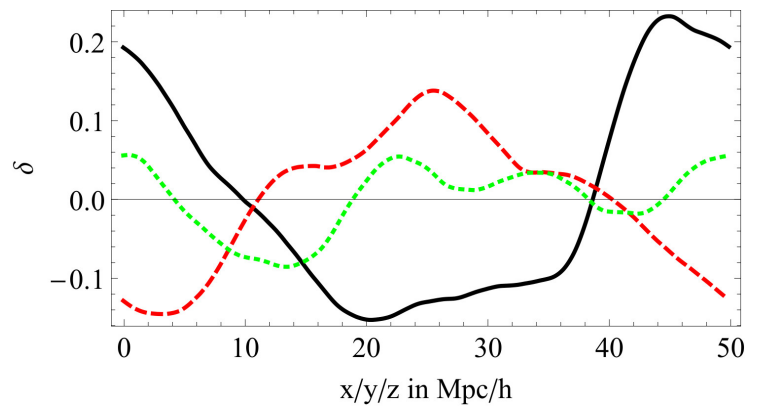

Fig. 9. Projected dark matter density along the $x$ - $/ y$ - $/ z$-axis (black solid/red dotted/green dashed line), smoothed on a scale of $10 \mathrm{Mpc} / \mathrm{h}$ for $z=4$.

velocity distribution reflects the density structure in the box, as can be seen by comparison with Fig. 9.

\subsubsection{Density}

As can be seen in the top-left plot in Fig. 5, larger overdensities are correlated with lower observed fractions. The effect is quite strong with about $30 \%$ in amplitude. We interpret this as a result of diffuse scattering around halos in overdense regions. Interestingly, the signal is highly suppressed if we switch off either the Hubble flow or the peculiar velocity field (see Fig. 6 , upper left subplot). Turning off the velocity field renders the spectra nearly symmetric. Photons that leave the ISM are not subject to further scattering, because neither local gas flows nor Hubble expansion can shift the photons back into the line center. This interpretation is also supported by calculations of the mean optical depth of the box. While in the line center, the optical depth (assuming a temperature of $2 \times 10^{4} \mathrm{~K}$ and taking the mean HI density from the simulation volume) is still $\tau \sim 10^{2}$, a shift of $1 \AA$ (in the local frame at $z=4$ ) reduces the optical depth to $\tau \sim 10^{-3}$. This also makes clear why velocity fields are so crucial in the radiative transfer.

When we turn on the peculiar velocity, the signal remains weak, but there is a slight decrease in observed fraction for halos in underdense regions. We interpret this as a result of the small halos being domimant in these underdense regions. The ISM in

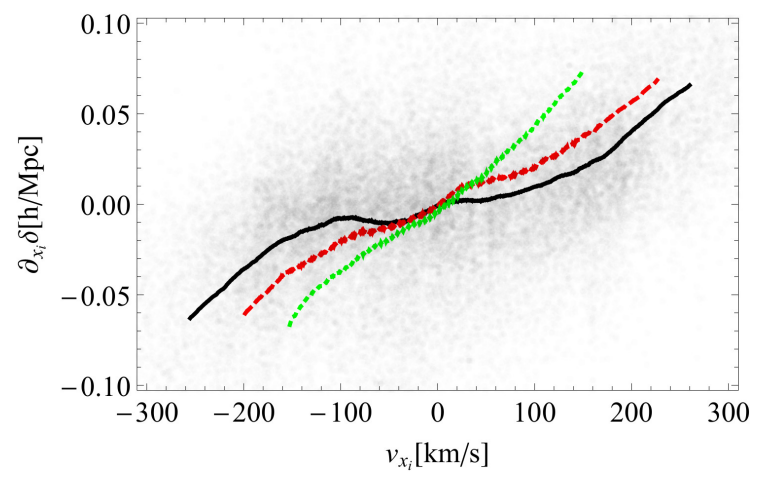

Fig. 10. Correlation between the line-of-sight density gradient and the line-of-sight velocity for all three lines of sight at $z=4, x$-axis (black solid), $y$-axis (red dashed) and $z$-axis (green dotted). The gray dots show the scatter, each point representing one halo (for the $x$-axis data). See text for details.

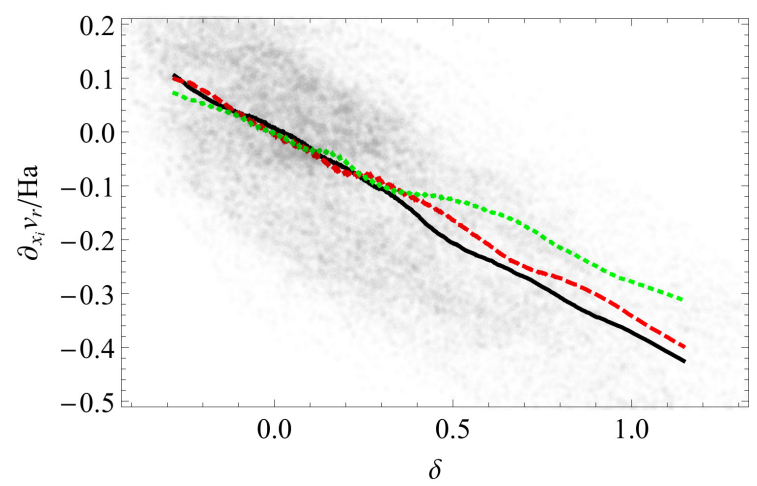

Fig. 11. Correlation between the density and the line-of-sight velocity gradient for all three lines of sight at $z=4, x$-axis (black solid), $y$-axis (red dashed), and $z$-axis (green dotted). The gray dots show the scatter, each point representing one halo (for the $x$-axis data). See text for details.

small halos does not push the photons as far out into the wings as in the larger ones, so the probability of being scattered in the IGM is increased. Also switching on the Hubble flow leads to our fiducial case: the Hubble flow together with the peculiar velocities leads to more numerous scatterings in overdense regions compared to the underdense regions.

\subsubsection{Line-of-sight velocity gradient}

The top right plot shows the correlation between line-of-sight velocity gradients and observed fractions. Here, we obtain qualitatively the same result as ZCTM10, although with a much lower amplitude; larger velocity gradients lead to a higher observed fraction. As has been discussed above, one can see that the effects of density and velocity gradient are anti-correlated as expected from linear theory (cf. Fig. 11). For this reason, there is no meaningful way to completely disentangle the two physical mechanisms. However, the dominant effect from the velocity gradient can be understood because a higher velocity gradient corresponds to a higher local effective Hubble flow. Since most of the photons that leave a halo are blue, the higher the effective 
C. Behrens and J. Niemeyer: Effects of Lyman-alpha scattering in the IGM on clustering statistics of Lyman-alpha emitters

Hubble flow, the faster the photons are transported through the line center and into the red wing. If we turn off the Hubble flow, the effect is reversed: a positive local gradient suppresses the emitters. In this case it might be caused by the redshifting through the line center taking place on a much larger length scale because the peculiar velocity gradient is much smaller than the Hubble flow.

It is worth pointing out that while in our simulations the amplitude of the correlation with line-of-sight velocity gradient is of the same order of magnitude as for the other correlations examined, ZCTM10 find a larger amplitude for the line of sight velocity gradient by about one order of magnitude. There are several factors that might be at play here. First of all, ZCTM10 worked at a higher redshift of $z=5.7$. The mean density is about 2.5 times higher at that redshift and therefore, the optical depth in the IGM is naturally higher, leading to more scatterings in the diffuse large-scale environment. This might leave a stronger imprint of the bulk velocity fields (and their spatial evolution) in the observed fraction. Secondly, whereas ZCTM10 semi-analytically map the baryons onto the results of a pure $\mathrm{N}$-body simulation, our gas distribution follows from a highresolution hydrodynamical simulation that includes the effects of nonlinear in- and outflows which reduce the correlations with flows on linear scales. Additionally, we resolve the ISM at least marginally, leading to a large number of scatterings in the dense regions where the photons are emitted. After being processed through the ISM, the photons have already been shifted from the line center to some extent. This also leads to less scattering in the IGM, further reducing the impact of the large-scale velocity field on the observed fraction.

\subsubsection{Line-of-sight density gradient}

This correlation is shown in the bottom-left plot of Fig. 5 . Because of the orientation of the observer towards the box, negative values here indicate that the density decreases in the direction of the observer. This makes the general trend in the plot plausible: observed fractions are lower if there is an intervening large-scale overdensity region between the emitter and the observer. The effect becomes smaller when turning off Hubble flow and peculiar velocities, as can be seen in the lower-left plot of Fig. 6. This is probably because the density of the environment becomes less important, as discussed in the preceding paragraphs. In contrast to ZCTM10, we find a prominent peak in the density gradient signal, at a value of around zero. We attribute this effect to the fact that halos with a density gradient of $\sim 0$ are predominantly located inside lower density regions and voids. Because of the correlation of higher observed fractions with lower densities, those halos have a higher observed fraction. Analysis shows that these low density halos $(\delta<0)$ populate the region around $\partial_{x_{i}} \delta \sim 0$, and that their mean observed fraction is about $6 \%$ higher than the mean observed fraction of halos in denser regions. The mean density at the location of halos with a density gradient between -0.03 and $0.0 \mathrm{~h} / \mathrm{Mpc}$, for example, is reduced by a factor of $50 \%$ compared to the full sample. Additionally, one can also notice that large absolute density gradients correspond to halos that are near the large-scale overdensity, and therefore in a region where the density is increased. For example, the mean large-scale overdensity for halos with a density gradient $<-0.08 \mathrm{~h} / \mathrm{Mpc}$ is roughly twice the mean of the whole sample. Since this increases the optical depth also for the halos on the near side of the halo, it could also account for the dip at large negative density gradients. This hypothesis is also supported because the effect does not fully vanish when the velocity field is set to zero, cf. Fig. 6 .

\subsubsection{Line-of-sight velocity}

In the bottom-right plot, the correlation between line-of-sight velocities and observed fractions is shown. The box is orientated so that halos moving into the direction of the observer have positive velocities. The plot's shape and the peak around zero stay the same even if we turn off the peculiar velocities in the simulation, as can be seen in the lower-right plot in Fig. 6. This indicates that the correlation is dominated by the density gradient, which looks nearly the same as the velocity signal when we turn off peculiar velocities. This is quite plausible since the density gradient and velocity fields are highly correlated, cf. Fig. 10. With Hubble flow and peculiar velocities switched on, we see a much larger amplitude and a strong suppression at positive velocities. Since halos with positive velocity move towards the observer while those with negative velocities recede, we interpret this as a consequence of the Hubble flow that suppresses blue halos more strongly than the red ones.

\subsection{Evolution with redshift}

In Fig. 7, the results for the dark matter correlation are shown for the redshift $z=2,3,4$. For this part of the analysis, the smoothing scale of the dark matter particles was adjusted to stay in the linear regime at lower redshifts. As has been discussed in a preceding section, the detection limit and the prescription of the intrinsic luminosity are somewhat arbitrary. Since the total mean density of the universe increases with redshift, we get different mean observed fractions at lower redshifts. At redshift 4, we have a total observed fraction of $30 \%$ at redshift 2 it has risen to above $80 \%$. For individual emitters, the observed fraction cannot be much larger than unity, so a higher total observed fraction can result in a compression of the correlation signal.

We find that correlations decline in amplitude with decreasing redshift. Since the mean density of the IGM decreases, the influence of the environment of the halos weakens. For the correlation of the observed fraction with the line-of-sight velocity, the drop of the Hubble rate from $\sim 400 \mathrm{~km} \mathrm{~s}^{-1} / \mathrm{Mpc}$ at $z=4$ to $\sim 200 \mathrm{~km} \mathrm{~s}^{-1} / \mathrm{Mpc}$ at $z=2$ further reduces the influence of the Hubble flow on the observed fractions from halos that move towards the observer $\left(v_{\mathrm{r}}>0\right)$.

While for the density gradient and the line-of-sight velocity, this decline in amplitude preserves the overall trend, for the density and the line-of-sight velocity gradient the lowest redshift $z=2$ shows a slight turnaround. For this redshift, halos in dense regions and in regions with a smaller velocity gradient are preferred by a few percentage points. One possible explanation is that the medium in dense regions is hotter at this later stage of structure formation and, therefore, contains less neutral gas. Since overdensities and low velocity gradients are coupled, this also affects the velocity gradient correlation.

In Table 1, we show the results for linear fits to the general trends seen in the correlations. They were calculated ignoring the dip on the left side in the density gradient and velocity plots (i.e., the left edge of the velocity- and density-gradient range). Because of the nonlinearity of the density correlation, the fitted value for this plot strongly depends on the chosen range for the fit. We obtained our fitted value by ignoring the steep decline below $\delta=0$. 
Table 1. Evolution with redshift.

\begin{tabular}{lccc}
\hline \hline & $z=4$ & $z=3$ & $z=2$ \\
\hline$\partial_{\delta} \Delta \epsilon$ & -0.48 & -0.15 & -0.05 \\
$\partial_{\partial_{\mathrm{r}} \delta} \Delta \epsilon$ & -3.0 & -1.94 & -0.78 \\
$\partial_{v_{\mathrm{r}}} \Delta \epsilon$ & $1.9 \times 10^{-3}$ & $1.1 \times 10^{-3}$ & $3.0 \times 10^{-4}$ \\
$\partial_{\partial_{\mathrm{r}} v_{\mathrm{r}}} \Delta \epsilon$ & 0.41 & 0.2 & -0.08 \\
\hline
\end{tabular}

\subsection{Effects on the two-point correlation function}

We calculate the two-point correlation function (2PCF) for our simulation data following Landy \& Szalay (1993) using a standard estimator that is frequently written as:

$\xi\left(r_{\perp}, \pi\right)=\frac{D D-2 D R+R R}{R R}$,

where $D D$ is the pair count of galaxies in the simulations, $R R$ is the pair count of random positions drawn from a uniform distribution, and $D R$ is the count for pairs consisting of one random location and one galaxy, all separated by a distance $\pi$ along the line of sight and $r_{\perp}$ orthogonal to that.

The real space $3 \mathrm{D}-2 \mathrm{PCF}$ as a function of line of sight separation $(\pi)$ and orthogonal separation $\left(r_{\perp}\right)$ is shown in Fig. 12 for halos (left), LAEs (middle) and a shuffled LAE sample (right) for our fiducial case with the $\operatorname{Ly} \alpha$ detection limit set as explained above. The shuffled LAE sample was constructed following ZTCM11 by randomly shuffling the properties of the simulated LAEs (S-LAE) to get rid off any correlation between apparent luminosity/observed fraction and the spatial location within the box. For all three samples, the number density was fixed to $4 \times 10^{-2} \mathrm{Mpc}^{-3} \mathrm{~h}^{3}$ which corresponds to a (apparent) luminosity threshold of $0.6 \times 10^{42} \mathrm{erg} / \mathrm{s}$ for the LAE/S-LAE sample and a mass threshold of $3.6 \times 10^{10} M_{\odot}$ for the halos. The redshift is $z=4$ for which we obtained the largest amplitude in correlations (see above). Near to an orthogonal separation of $\sim 0$, the plot shows relatively strong fluctuations. Those are induced by the small sample size near $r_{\perp} \sim 0$ and by the finite resolution of our output grid, resulting in source blending; the projected distance of LAEs in this region is too small to disentangle them. We do not find a significant deformation of the 2PCF. ZCTM11 report an strong elongation pattern in the $2 \mathrm{PCF}$ along the lineof-sight direction which they attribute to a correlation between observed fraction and line-of-sight velocity gradient. In Fig. 13, we plot the relative deviation of the LAEs' $2 \mathrm{PCF}$ with respect to the shuffled sample, $\xi_{\text {diff }}=\left(\xi_{\mathrm{LAE}}-\xi_{\mathrm{S}-\mathrm{LAE}}\right) / \xi_{\mathrm{S}-\mathrm{LAE}}$. As can be seen, there is no indication of a deformation. We also tried different higher detection limits and luminosity thresholds, but did not find a significant elongation pattern. Also, the correlation with line-of-sight velocity gradient is not stronger with a higher detection threshold. We conclude that in contrast to ZCTM11, we do not find a significant elongation in the line-of-sight direction.

In addition to the visual inspection of the $2 \mathrm{PCF}$ contours, we computed its quadrupole moment in order to quantitatively verify the absence of a distortion effect from Ly $\alpha$ RT. In Fig. 14, we show the normalized quadrupole $Q(s)$ (e.g., Chuang \& Wang 2013) as defined by

$$
Q(s)=\frac{\xi_{2}(s)}{\xi_{0}(s)-3 / s^{3} \int_{0}^{s} \xi_{0}\left(s^{\prime}\right) s^{\prime 2} \mathrm{~d} s^{\prime}}
$$

where $\xi_{0}(s) / \xi_{2}(s)$ is the monopole/quadrupole contribution as a function of $s=\sqrt{\pi^{2}+r_{\perp}^{2}}$.
Even for a threshold of $1 \times 10^{-17} \mathrm{erg} \mathrm{s}^{-1} \mathrm{~cm}^{-2} \operatorname{arcsec}^{-2}$ which removes $90 \%$ of all emitters, no significant signal was found in the magnitude of the quadrupole moment.

Again, this result can at least in part be attributed to our lower redshift. The amplitude of the selection effect induced by the RT and the observation threshold strongly depends on the optical depth in the IGM. The denser and more neutral IGM in ZCTM10/11 results in a stronger dimming of the central regions of a source, leading to a lower observed fraction. By tuning the observation threshold alone, this cannot be mimicked.

On the other hand, the linear analysis by Wyithe \& Dijkstra (2011), evaluated with coefficients estimated from our results, indicates that our box size may be insufficient to measure a signal. Specifically, Wyithe \& Dijkstra (2011) present an analytical model for estimating the impact of the radiative transfer on the clustering signal. The parameters $C_{v}$ and $C_{\rho}$ defined in their Eqs. (12) and (13) measure how the relative transmission of the IGM is affected by fluctuations in the velocity gradient and density field, respectively. These quantities are comparable by construction to $\partial_{\partial_{\mathrm{r}} v_{\mathrm{r}}} \Delta \epsilon$ and $\partial_{\delta} \Delta \epsilon$ used above.

We compare with our results in Table 1 by computing $C_{v}$ and $C_{\rho}$ with values estimated from our data, namely the fraction of photons scattered in the IGM, $F \approx 0.7$, the mean IGM optical depth, $\tau_{\text {IGM }} \approx 1.5$, and the luminosity function power law index, $\beta=2.2$. We obtain $C_{v} \approx 0.6$ and $C_{\rho} \approx-1$ from the analytic model, which is broadly consistent with our values for $\partial_{\partial_{\mathrm{r}} v_{\mathrm{r}}} \Delta \epsilon$ and $\partial_{\delta} \Delta \epsilon$. At this level of $C_{v}$, the analysis of Wyithe \& Dijkstra (2011) would suggest a small but noticeable deformation of the 2PCF. We consequently cannot rule out that the absence of a signal in our results is affected by our limited statistics.

\subsection{Impact of inclination on the observed fraction}

In Fig. 15, the correlation between observed fraction and the inclination angle between the line-of-sight and the angular momentum of the dark matter halo is shown for all three redshifts. For this plot, we use the full sample of simulated LAEs. We use the dark matter angular momentum as a proxy for the disk orientation in order to avoid ambiguities in the definition of the disk plane, given the marginal resolution of galactic disks in the MareNostrum simulation. Of course, the scatter between disk orientation and halo angular momentum reduces the signal from disk orientation effects on the Ly $\alpha$ observed fraction as seen, for instance, in Bett (2012). On the other hand, this choice provides a more direct measure of the impact of halo tidal alignment on the clustering statistics, to be discussed below. For simplicity, we will refer to galaxies viewed along the direction of their halo angular momentum as face-on, even though the viewing direction may not be exactly normal to the disk plane.

For face-on galaxies, the observed fraction is increased by $\sim 15 \%$ with respect to edge-on galaxies. This is intuitive, since photons preferentially escape perpendicular to the disk because of the reduced optical depth compared to the path through the disk plane. Since this is a local effect, it is independent of the chosen line of sight and is not affected by switching off/on the peculiar velocity field or the Hubble flow.

Strong inclination dependence has also been found by various groups (e.g., Laursen \& Sommer-Larsen 2007; Yajima et al. 2012; Verhamme et al. 2012) in simulations of isolated disk galaxies. Their results also showed a strong sensitivity on the morphology of the ISM, with a denser and clumpier structure exhibiting a significantly more pronounced dependence on viewing angle. In order to assess the full extent of LAE emission characteristics as a function of inclination angle, high-resolution 
C. Behrens and J. Niemeyer: Effects of Lyman-alpha scattering in the IGM on clustering statistics of Lyman-alpha emitters
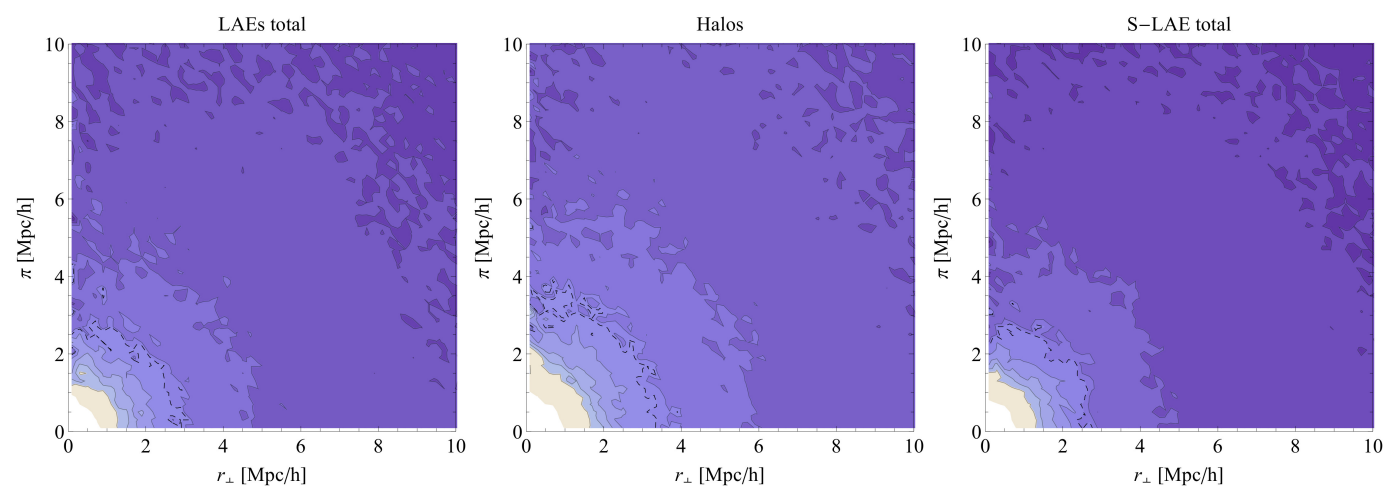

Fig. 12. $2 \mathrm{PCF}$ as a function of line of sight $(\pi)$ and perpendicular distance $\left(r_{\perp}\right)$ for halos (left plot), observed LAEs (middle), and S-LAE sample (right) in real space at $z=4$. The dashed contours correspond to $\xi=1$, contours are separated by 0.4 . The number density of all threshold samples is $4 \times 10^{-2} \mathrm{Mpc}^{-3} \mathrm{~h}^{3}$. The plots were averaged over three different lines of sight to reduce cosmic variance.

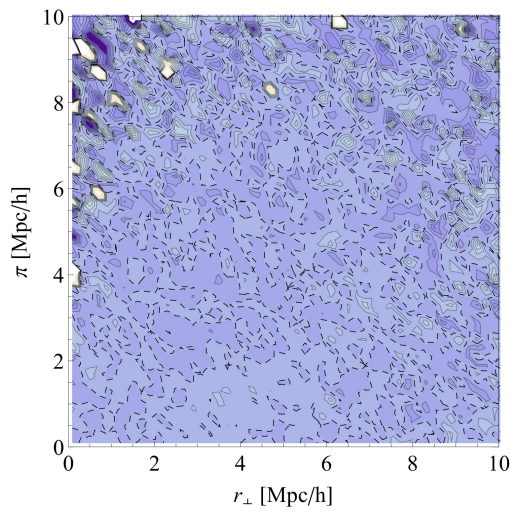

Fig. 13. Ratio of the $2 \mathrm{PCF}$ of S-LAE and LAEs $\xi_{\text {diff }}=\left(\xi_{\mathrm{LAE}}-\right.$ $\left.\xi_{\mathrm{S}-\mathrm{LAE}}\right) / \xi_{\mathrm{S}-\mathrm{LAE}}$. Contours are separated by $15 \%$; the dashed contour corresponds to a value of 0 . Number densities and thresholds are the same as in Fig. 12.

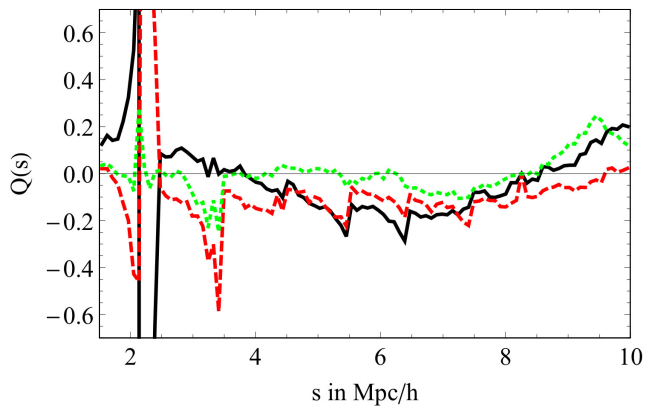

Fig. 14. $\mathrm{Q}$ (s) for $z=4$ halo sample (black solid), LAE sample (red dashed), and S-LAE sample (green dotted line). Number density is $4 \times$ $10^{-2}$. The spikes at $2-3 \mathrm{Mpc} / \mathrm{h}$ are due to poles.

simulations which provide a fair representation of the ISM morphology are required. Our results, based on a simulation with marginal spatial resolution of galaxies which results in a very smooth ISM structure, can therefore only provide a lower bound on the expected magnitude of the effect.

We do not find a significant evolution of the inclination dependence with redshift. This is surprising, since one might expect the disk-like shape to become more prominent at lower redshift because of the higher total mass in the ISM. Further investigation is needed to resolve this issue, but one has to keep in mind that comparisons of the signal's amplitude can be difficult between different redshifts (cf. Sect. 4.3).

The signature of orientation dependence and tidal alignment on redshift space distortions (RSD) has been analyzed by Hirata (2009) who concludes that the effect is degenerate with gravitationally induced RSD (i.e., the Kaiser effect, Kaiser 1987) and may amount to several percentage points for reasonable assumptions about alignment and inclination dependence of the observed flux. However, it is easy to see that the coefficient that measures the orientation dependence (named $\psi$ in Hirata 2009) can be made much larger if one assumes a very steep transition from edge-on to face-on flux, such as the one observed by Verhamme et al. (2012) in their case G2. The smoothness of the transition seen in our results (Fig. 15) can be attributed to two effects that have already been mentioned above. First, the spread of disk orientations with respect to halo angular momentum washes out the overall signal, and second, the spatial resolution is inadequate to capture the full extent of the expected orientation dependence. While the former is physical and will be present in real data, the latter is an artifact of our method.

These results are highly suggestive that LAEs can provide a sensitive probe of gravitationally induced tidal alignment. This could reduce the accuracy of growth factor measurements from surveys like HETDEX; however, additional information from the galaxy bispectrum can break the degeneracy (cf. Krause \& Hirata 2011), but it also offers the attractive opportunity to search for tidal alignment in LAE survey data and to test the predictions of CDM structure formation. For instance, recent results from large $\mathrm{N}$-body simulations show an alignment along large-scale structure filaments for lower-mass halos, whereas the angular momenta of high-mass halos preferentially align perpendicular to the direction of filaments (Codis et al. 2012). Further work is needed to explore the potential of LAEs as tracers of cosmic alignment.

\section{Conclusions}

Our numerical analysis clearly shows that resonant scattering in the CGM and IGM can strongly suppress observed Ly $\alpha$ fluxes. We find mean observed fractions between $30 \%$ at $z=4$ and around $80 \%$ at $z=2$. We stress that we do not include destruction by dust, hence the suppression in flux only results from anisotropic escape of photons from their halos and diffuse 
A\&A 556, A5 (2013)

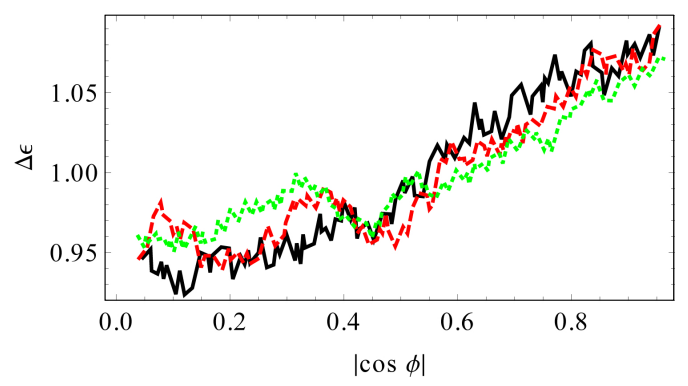

Fig. 15. Correlation between the relative observed fraction and the inclination of the halos with respect to the observer. An inclination of 1 corresponds to a face-on emitter, 0 corresponds to edge-on. The lines show data from $z=4$ (black), $z=3$ (red), and $z=2$ (green).

scattering in the IGM. These results are consistent with Laursen et al. (2011)

We do find correlations between the large-scale density and velocity fields and the observed $\operatorname{Ly} \alpha$ fraction. This broadly confirms the results of ZCTM10 who report a much stronger effect at higher redshifts. Apart from their overall smaller amplitude, the correlations seen in our work differ from those found by ZCTM10 in their relative strength. Whereas the velocity gradient has by far the strongest effect in their results, in our case it is comparable in magnitude to the correlations of $\operatorname{Ly} \alpha$ observed fraction with large-scale density, density gradient, and velocity fields. This appears more natural to us since density and velocity gradient are correlated via the continuity equation.

All of the correlations with large-scale fields that we found have plausible interpretations in terms of resonant scattering with neutral hydrogen modulated by density and Doppler shift. Owing to the strong correlations between the density and velocity fields in the linear regime, it is not always possible to unambiguously identify the dominant effect. By artificially turning off the peculiar velocity and Hubble flow terms in the scattering cross sections, we were able to separate the effects of density and velocity to some extent. The results are consistent with intuitive expectations: the effect of large-scale overdensity is largest and similar to the velocity gradient, followed closely by velocity and density gradient.

For all correlations except orientation dependence we find a strong decrease of their amplitude from redshift 4 to 2 . Despite the strong impact of the radiation transport on the observed fluxes, we do not reproduce the clustering signal found by ZCTM11. Even at $z=4$ where the correlations are strongest, we fail to detect a significant change in the 3D 2PCF. Although the lower redshift of our studies is expected to reduce the clustering signal, a comparison with the analytical model by Wyithe \& Dijkstra (2011) suggests that this is not the full explanation. The values we find for the dependence of the observed flux on the large-scale velocity gradient would, according to their model, lead to a small but detectable deformation in the 2PCF. It is therefore plausible that the limited statistics due to our finite box size are partly responsible for our failure to detect nongravitational clustering from Ly $\alpha$ RT effects.

We also found a distinctive correlation between the Ly $\alpha$ observed fraction and the angular momentum of the dark matter halo, which we interpret as a signal of the orientation dependence of LAE fluxes. Although the amplitude of the signal is only $\sim 15 \%$ in our numerical analysis, we assume that it can be substantially larger in reality as our results are limited by poor spatial resolution of the ISM. In this case, partial alignment of halo spins with the large-scale tidal field may give rise to contaminating contributions to redshift space distortions (Hirata 2009; Krause \& Hirata 2011). On the other hand, our results combined with recent high-resolution simulations of LAEs (Verhamme et al. 2012) suggest that LAEs provide a sensitive observational probe of tidal alignment.

Acknowledgements. This work was supported by the DFG SFB 963/1, project A13. We acknowledge the Horizon collaboration for making the MareNostrum data set available to us. We thank Mark Dijkstra for many fruitful discussions and comments, and Eichiro Komatsu for helpful suggestions.

\section{References}

Adams, J. J., Hill, G. J., \& MacQueen, P. J. 2009, ApJ, 694, 314 Adams, J. J., Blanc, G. A., Hill, G. J., et al. 2011, ApJS, 192, 5 Adams, J. J., Blanc, G. A., Hill, G. J., et al. 2011, Ap Barnes, L. A., Haehnelt, M. G., Tescari, E., \& Viel, M. 2011, MNRAS, 416, 16 Bett, P. 2012, MNRAS, 420, 3303

Cantalupo, S., Porciani, C., Lilly, S. J., \& Miniati, F. 2005, ApJ, 628, 61

Chuang, C.-H., \& Wang, Y. 2013, MNRAS, 426, 226

Codis, S., Pichon, C., Devriendt, J., et al. 2012, MNRAS, 427, 18

Dayal, P., \& Ferrara, A. 2012, MNRAS, 421, 2568

Dijkstra, M., \& Wyithe, S. 2010, MNRAS, 408, 11

Dijkstra, M., Haiman, Z., \& Spaans, M. 2006, ApJ, 649, 14

Dijkstra, M., Lidz, A., \& Wyithe, J. S. B. 2007, MNRAS, 377, 1175

Eisenstein, D. J., \& Hut, P. 1998, ApJ, 498, 137

Faucher-Giguere, C. A., Keres, D., Dijkstra, M., Hernquist, L., \& Zaldarriaga, M. 2010, ApJ, 725, 29

Forero-Romero, J. E., Yepes, G., Gottlöber, S., et al. 2011, MNRAS, 415, 3666 Gay, C., Pichon, C., Borgne, D. L., et al. 2009, MNRAS, 404, 18 Greig, B., Komatsu, E., \& Wyithe, J. S. B. 2013, MNRAS, 431, 19

Guaita, L., Gawiser, E., \& Padilla, N. 2010, ApJ, 714, 1

Hansen, M., \& Peng Oh, S. 2006, New Astron. Rev., 50, 58

Harrington, J. P. 1974, MNRAS, 166, 373

Hirata, C. M. 2009, MNRAS, 399, 1074

Iliev, I. T., Shapiro, P. R., McDonald, P., Mellema, G., \& Pen, U.-L. 2008, MNRAS, 391, 63

Kaiser, N. 1987, MNRAS, 227, 1

Katz, N., Weinberg, D. H., \& Hernquist, L. 1996, ApJS, 105, 19

Kobayashi, M. A. R., Inoue, Y., \& Inoue, A. K. 2013, ApJ, 763, 12

Krause, E., \& Hirata, C. 2011, MNRAS, 410, 10

Landy, S. D., \& Szalay, A. S. 1993, ApJ, 412, 64

Laursen, P., \& Sommer-Larsen, J. 2007, ApJ, 657, L69

Laursen, P., Razoumov, A. O., \& Sommer-Larsen, J. 2009a, ApJ, 696, 853

Laursen, P., Sommer-Larsen, J., \& Andersen, A. C. 2009b, ApJ, 704, 1640

Laursen, P., Sommer-Larsen, J., \& Razoumov, A. O. 2011, ApJ, 728, 16

Lee, J.-S. 1974, ApJ, 192, 465

Nagamine, K., Ouchi, M., Springel, V., \& Hernquist, L. 2010, PASJ, 62

Ocvirk, P., Pichon, C., \& Teyssier, R. 2008, MNRAS, 390, 15

Ouchi, M., Shimasaku, K., Akiyama, M., et al. 2008, ApJS, 176, 301

Press, W. H., Teukolsky, S. A., Vetterling, W. T., \& Flannery, B. P. 2007,

Numerical Recipes 3rd edn. The Art of Scientific Computing (Cambridge University Press)

Schaerer, D., Hayes, M., Verhamme, A., \& Teyssier, R. 2011, A\&A, 531, A12

Shimizu, I., Yoshida, N., \& Okamoto, T. 2011, MNRAS, 418, 2273

Tasitsiomi, A. 2006, ApJ, 645, 792

Teyssier, R. 2002, A\&A, 385, 337

Verhamme, A., Dubois, Y., Blaizot, J., et al. 2012, A\&A, 546, 13

Wang, J.-X., Malhotra, S., Rhoads, J. E., Zhang, H.-T., \& Finkelstein, S. L. 2009 ApJ, 706, 762

Whitney, B. A. 2011, BASI, 38, 26

Wyithe, S., \& Dijkstra, M. 2011, MNRAS, 415, 24

Yajima, H., Li, Y., \& Zhu, Q. 2012, ApJ, submitted

Zheng, Z., \& Miralda-Escude, J. 2002, ApJ, 578, 33

Zheng, Z., Cen, R., Trac, H., \& Miralda-Escude, J. 2010, AJ, 716, 28

Zheng, Z., Cen, R., Trac, H., \& Miralda-Escude, J. 2011a, ApJ, 726, 31

Zheng, Z., Cen, R., Weinberg, D., Trac, H., \& Miralda-Escudé, J. 2011b, ApJ, 739,62

Pages 11 to 13 are available in the electronic edition of the journal at http://wWw . aanda.org 
C. Behrens and J. Niemeyer: Effects of Lyman-alpha scattering in the IGM on clustering statistics of Lyman-alpha emitters

\section{Appendix A: The physics of Ly $\alpha$ transport}

\section{A.1. General}

Ly $\alpha$ photons emitted in starforming regions of a galaxy undergo resonant scatterings that result in a stochastic movement in space and frequency. As in many previous studies, we denote the frequency of a Ly $\alpha$ photon with the dimensionless quantity

$x=\frac{v-v_{0}}{v_{\mathrm{D}}}$,

where $v_{\mathrm{D}}$ is the Doppler frequenc; $v_{\mathrm{D}}=\frac{v_{\mathrm{th}} \nu_{0}}{c}$ with the most probable thermal velocity of the atoms $v_{\mathrm{th}}=\left(\frac{2 k_{\mathrm{B}} T}{m_{\mathrm{H}}}\right)^{1 / 2}$ and all other symbols have their usual meaning.

A non-zero bulk velocity of the gas $\boldsymbol{v}$ can be taken into account easily by performing a first-order Lorentz transformation into the restframe of the macroscopic gas motion:

$x^{\prime}=x-\frac{\boldsymbol{v} \cdot \boldsymbol{n}}{v_{\mathrm{th}}}$.

Following Dijkstra et al. (2006), we denote quantities measured in the restframe of macroscopic bulk velocity with a prime. If not mentioned otherwise, quantities are measured in the frame of an observer which is at rest with respect to the center of mass (but notice the remarks on the Hubble flow in Appendix B). Here, $\boldsymbol{n}$ is the direction of the photon.

\section{A.2. Absorption and reemission}

The scattering cross section of a $\operatorname{Ly} \alpha$ photon can be written as

$\sigma_{\mathrm{L}}=f_{12} \frac{\sqrt{\pi} \mathrm{e}^{2}}{m_{\mathrm{e}} c v_{\mathrm{D}}} H\left(a, x^{\prime}\right)$

with $f_{12}$ the Einstein coefficient and $H\left(a, x^{\prime}\right)$ the Voigt profile that depends on the dampening parameter $a=\frac{\Delta v}{2 v_{\mathrm{D}}}$. $\Delta v$ is the natural line width; Therefore, the optical depth $\tau$ for Ly $\alpha$ traveling a distance $l$ with frequency $x$ through a gas with neutral hydrogen number density $n_{\mathrm{H}}$ is

$\tau=\int_{0}^{l} \sigma_{\mathrm{L}} n_{\mathrm{H}} \mathrm{d} l^{\prime}$.

The probability $P$ of a photon of passing through an optical depth $\tau$ without being absorbed is equal to

$P=\mathrm{e}^{-\tau}$.

Neutral hydrogen atoms on which the scatterings occur follow a specific velocity distribution because of their random thermal velocity and the macroscopic gas velocity, since photons are $\mathrm{red} / \mathrm{blueshifted} \mathrm{in} \mathrm{the} \mathrm{frame} \mathrm{of} \mathrm{the} \mathrm{scattering} \mathrm{atom.} \mathrm{It} \mathrm{is} \mathrm{conve-}$ nient to split the thermal velocity into components parallel and orthogonal to an incoming photon. The PDF of the parallel component is

$P\left(v_{z}\right)=\frac{a \mathrm{e}^{-v_{z}^{2}}}{\pi\left[\left(x^{\prime}-u_{z}\right)^{2}+a^{2}\right]} H^{-1}$.

The other two components orthogonal to the direction of the infalling photon follow a Gaussian distribution.

Absorption is quickly followed $\left(\Delta t \sim 10^{-9} \mathrm{~s}\right)$ by reemission.

The frequency of the reemitted photon depends on the scattering atom's velocity because the scattering is coherent in the atom's restframe, but not necessarily in the reference frame of an observer. If $\boldsymbol{v}_{\mathrm{a}}$ denotes the atom's velocity in the frame of the observer, then the relation between the frequency of the infalling photon $x_{\mathrm{i}}$ and the reemitted photon $x_{\mathrm{r}}$ satisfies

$x_{\mathrm{r}}=x_{\mathrm{i}}+\frac{\boldsymbol{v}_{\mathrm{a}} \cdot\left(\boldsymbol{n}_{\mathrm{r}}-\boldsymbol{n}_{\mathrm{i}}\right)}{v_{\mathrm{th}}}$,

where $\boldsymbol{n}_{\mathrm{i}} / \boldsymbol{n}_{\mathrm{r}}$ is a unit vector in the direction of the infalling/reemitted photon. We neglect the recoil on the scattering atom here, because it has been shown to have no significant effect on the radiation transport (Zheng \& Miralda-Escude 2002). The distribution of the remission's direction is determined by a phase function. Depending on the frequency of the infalling photon, different phase functions have been proposed for the angular distribution of Ly $\alpha$ photons. As shown in Tasitsiomi (2006), for numerical simulations the differences between the phase function are quickly washed out by the resonant scatterings. For that reason we use an isotropic phase function that can be written as

$P\left(\boldsymbol{n}_{\mathrm{r}} \mid \boldsymbol{n}_{\mathrm{i}}\right)=$ const.

Since the frequency of the photons generally changes because of the scatterings, the photons perform a random walk in space and frequency (Harrington 1974). The cross section quickly decreases when a photon leaves the line center, so the mean free path will increase drastically for a photon left/right of the line center. Typically, photons leave an optical thick medium after a couple of scatterings on atoms to which they appear strongly redor blueshifted because in that case it is probable that the photon is reemitted with a frequency far away from the line center measured in the reference frame of the observer.

\section{Appendix B: LyS - A Ly $\alpha$ simulation code}

Our implementation of a Monte-Carlo code for $\operatorname{Ly} \alpha$ radiation transport is called LyS. It is capable of tracking photons in grids with AMR. LyS is OpenMP-parallelized, so on machines with multiple cores, each core can handle one photon at a time. Additionally, MPI was implemented to deal with the large data set of the MareNostrum galaxy formation simulation.

Similar to other codes, LyS solves the radiation transfer problem for individual photons via the following iterative algorithm:

1. Draw an optical depth $\tau_{0}$ exponentially distributed (Eq. (A.5));

2. Integrate the optical depth $\tau$ while the photon traverses a grid of gas cells (Eq. (A.4));

3. When $\tau$ equals $\tau_{0}$, a scattering point is reached. Draw the velocity of the scattering atom from the PDF in Eq. (A.6);

4. Calculate the new frequency and direction of the scattered photon according to Eqs. (A.7) and (A.8).

The iteration is stopped when a photon has traveled a quarter of the box length from its source. If it reaches a box boundary before traveling a quarter length, we apply periodic boundaries.

For the generation of the output, we use the so-called next event estimator or peeling-off method (Whitney 2011). At each scattering, we calculate the probability of the photon being reemitted into the direction of the observer and reaching the boundary of the box without additional scatterings. This might be thought of as sending out a tracer photon at each scattering event. The probability of reaching the observer is given by Eq. (A.5), where $\tau$ is now the optical depth along the line of sight from the scattering point to the boundary of the box. Since 


\subsection{Effects of Lyman-Alpha Scattering in the IGM on Clustering Statistics of Lyman-Alpha Emitters5}

A\&A 556, A5 (2013)

the frequency of the reemitted photon depends on the direction in which it is emitted, one has to assign the frequency for the tracer photon according to Eq. (A.7). The calculated probability is summed in the output array for each scattering. Assuming the observer is located in the direction of the positive $x$-axis, the array holds $n_{y} \times n_{z} \times n_{\lambda}$ bins for the $y$ - $z$-coordinate of the photon and the physical wavelength.

The line-of-sight integration used in the peeling-off method also applies periodic boundaries if the integration distance is less than a quarter of the box length (and stops the integration if this value is reached). This removes edge effects due to the finite extent of the box. One could also just skip sources near the boundaries, but this would result in the loss of many emitters, degrading the statistics. Physically, this corresponds to a flattening of the volume in the line-of-sight direction. This is plausible since the box is thin compared to the distance to the observer.

During the line-of-sight integration, tracer photons are redshifted according to the linear Hubble Law. Regular photons are also redshifted on their path through the volume. This is implemented by adding a term

$\boldsymbol{v}_{\mathrm{H}}=-H \boldsymbol{d}_{\mathrm{lsp}}$

to the bulk velocity in Eq. (A.2), where $\boldsymbol{d}_{\text {scat }}$ denotes the distance vector to the last scattering location of the photon and $H$ is the Hubble rate at the specific redshift. In this sense, each photon has its own frame of reference, in rest with respect to the observer's frame, but seeing a spherical velocity field centered on the last scattering location.

To find random numbers following the distribution in Eq. (A.6), we use the so-called rejection method (Press et al. 2007) in an implementation similar to Laursen et al. (2009a). The LyS implementation uses the acceleration scheme proposed by Ahn et al. (2002) which reduces the number of scatterings by skipping so-called core scatterings. This is done by cutting off the velocity distribution of the scattering atom below some value, which effectively forces photons to be scattered by atoms to which they appear far in the blue or red. If the frequency $|x|$ is below a critical frequency $x_{\mathrm{cw}}$, the components of a scattering atom's velocity that are perpendicular to the photon direction of flight are drawn via

$v_{\perp, 0}=\sqrt{x_{\mathrm{cw}}^{2}-\log R_{4}} \cos \left(2 \pi R_{5}\right)$

$v_{\perp, 1}=\sqrt{x_{\mathrm{cW}}^{2}-\log R_{4}} \sin \left(2 \pi R_{5}\right)$.

Here, the $R_{\mathrm{i}}$ are random numbers drawn from a uniform distribution.

In this paper, we focus on $\operatorname{Ly} \alpha$ radiation from star forming regions near the center of young galaxies. Taking into account the resolution of our simulation box, it is a good approximation to emit photons at the center of the halos. Following ZCTM10, we choose the intrinsic Ly $\alpha$ luminosity of a halo proportional to its star formation rate $R_{\mathrm{SF}}$, which is in turn a linear function of the halo mass $M_{\mathrm{h}}$ :

$$
\begin{aligned}
L_{\mathrm{i}} & =10^{42} R_{\mathrm{SF}} \frac{\mathrm{erg} \mathrm{s}^{-1}}{\mathrm{yr}^{-1} M_{\odot},} \\
R_{\mathrm{SF}} & =0.68 \frac{M_{\mathrm{h}}}{10^{10} M_{\odot}} \mathrm{yr}^{-1} .
\end{aligned}
$$

Both equations should vary with redshift. The cosmic star formation history reached its peak around redshift 2 (Kobayashi et al. 2013), and the intrinsic $\operatorname{Ly} \alpha$ luminosity should be modified by dust attenuation that is also a function of star formation

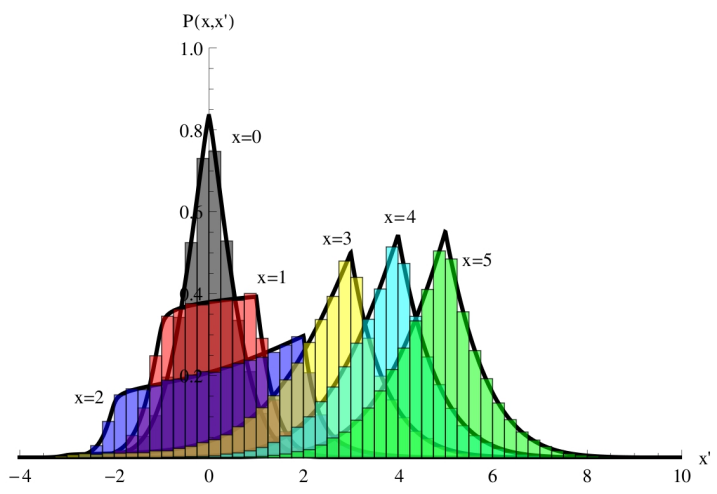

Fig. B.1. Comparison of the redistribution function calculated using the analytic solution by Lee (1974) (solid lines) with our code. Shown is the probability that a photon will be reemitted with a frequency $x^{\prime}$ when it has the frequency $x$ before the scattering occurs for some values of $x$.

history. We ignore this here, since from the perspective of our numerical simulation, the total intrinsic luminosity plays only the role of a normalization, especially because we are mostly interested in ratios between intrinsic and apparent luminosities. We also stress that we chose this specific model to be comparable to previous work, not because it is the relation predicted by the MareNostrum simulation. Photons are emitted with a frequency drawn from a Gaussian. Its width is determined by the viral temperature of the halo:

$T_{\mathrm{vir}}=\frac{G M_{\mathrm{h}} \mu m_{\mathrm{H}}}{3 k_{\mathrm{Bc}}}$.

Here, $R_{\text {vir }}$ denotes the virial radius of the halo and $\mu$ is the mean molecular weight. In this way, we include the velocity distribution of emitters that are gravitationally bound. Photons are emitted from halos with mass $\geq 5 \times 10^{9} M_{\odot}$. Since the range of masses and thus intrinsic luminosities is about 3 orders of magnitude, we follow ZCTM10 in applying a weighting procedure for the individual photons to reduce the total number of photons to compute. Each halo emits at least $n_{\min }=1000$ photons independently of its mass. In total, we run the RT for about $40000 / 49000 / 51000$ halos at redshift 4/3/2. To conserve the relative intrinsic luminosities, photons are given a massdependent weight.

\section{B.1. Code verification}

To verify the correctness of our radiative transfer code, we perform the standard tests from the literature. In Fig. B.1, the redistribution function $f\left(x, x^{\prime}\right)$ is shown, namely the probability for an infalling photon with frequency $x$ to be reemitted with a frequency $x^{\prime}$. In this test, only thermal motions of the scattering atom are considered. Overplotted are the analytical solutions by Lee (1974).

The standard test for Ly $\alpha$-codes, the so-called static sphere test, is shown in Fig. B.2 for various optical depths $\left(\tau_{0}=\right.$ $\left.10^{5} / 10^{6} / 10^{7}\right)$. In this test, photons are launched in the center an isothermal sphere of constant density. Overplotted is the analytic solution from Dijkstra et al. (2006). For these tests, the acceleration scheme was turned off. The static sphere test was also done with the activated acceleration scheme. It still resembles the analytical solution quite well. 
C. Behrens and J. Niemeyer: Effects of Lyman-alpha scattering in the IGM on clustering statistics of Lyman-alpha emitters

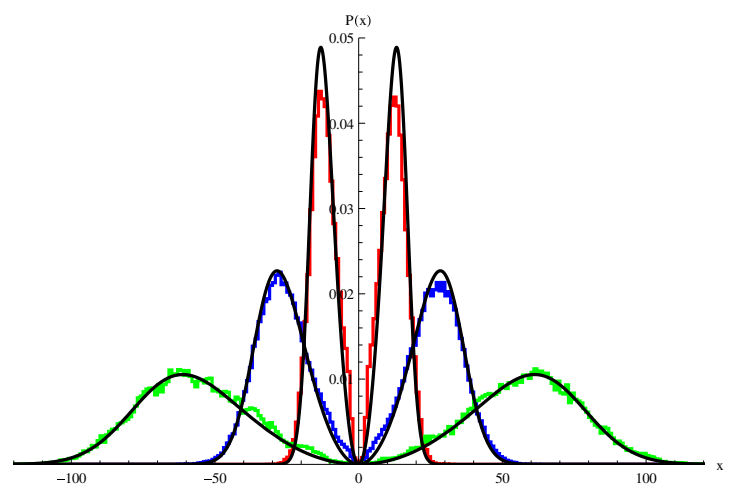

Fig. B.2. Comparison between the analytical solution (solid lines) of the spherical test case (see text) and the results obtained by LyS. Shown is the probability distribution of the escaping photons as a function of the dimensionless frequency $x$. The innermost peaks correspond to an optical depth of $10^{5}$, the outermost to $10^{7}$. The third case is for an optical depth of $10^{6}$.

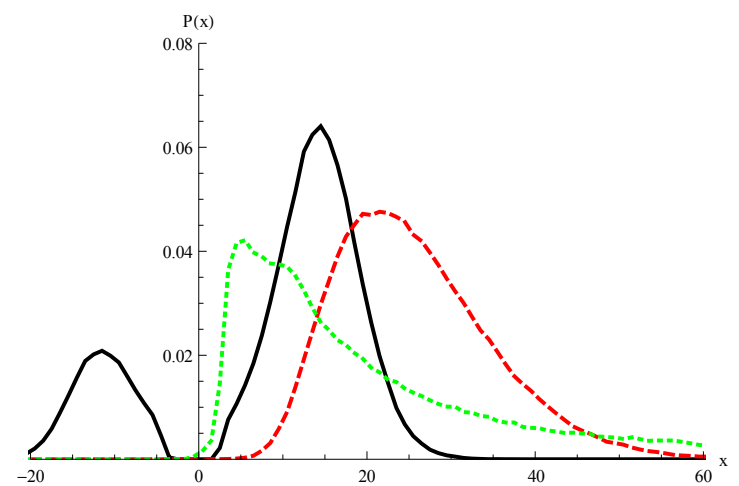

Fig. B.3. Dimensionless frequency distribution of photons escaping from an isothermal $\left(2 \times 10^{4} \mathrm{~K}\right)$ homogeneous sphere with column density of $2 \times 10^{20} N_{\mathrm{H}}$ from the center to the surface. A Hubble-like velocity prescription given by Eq. (B.7) is assigned. The different lines correspond to different maximum collapse velocities: $20 \mathrm{~km} \mathrm{~s}^{-1}$ (line), $200 \mathrm{~km} \mathrm{~s}^{-1}$ (dashed), $2000 \mathrm{~km} \mathrm{~s}^{-1}$ (dotted).

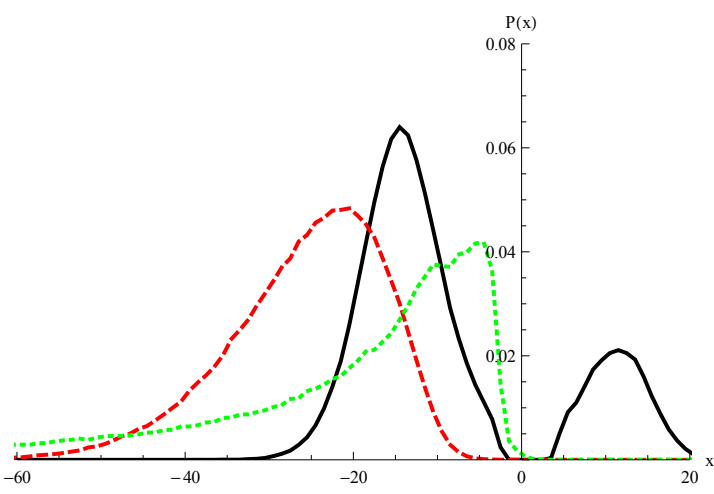

Fig. B.4. Same as Fig. B.3, but for an expanding sphere. The different lines correspond to the maximum expansion velocities: $20 \mathrm{~km} \mathrm{~s}^{-1}$ (line), $200 \mathrm{~km} \mathrm{~s}^{-1}$ (dashed), $2000 \mathrm{~km} \mathrm{~s}^{-1}$ (dotted).

For Figs. B.3 and B.4, the static sphere problem was modified with a Hubble-like bulk velocity field. The gas was assigned a velocity

$\boldsymbol{v}_{\text {bulk }}=\frac{v_{\max } \boldsymbol{r}}{r_{\max }}$.

Where $v_{\max }$ is a certain constant maximum velocity $v_{\max }, \boldsymbol{r}$ the distance vector from the center of the sphere and $r_{\max }$ the distance from the center where $|\boldsymbol{v}|=v_{\max }$. Since there is no analytic solution for this test case available, we can only compare the results from other codes. The results from LyS are in good agreement with the plots in Faucher-Giguere et al. (2010) and Dijkstra et al. (2006). 
4.2 Beamed Ly $\alpha$ Emission through Outflow-driven Cavities 


\title{
Beamed Ly $\alpha$ emission through outflow-driven cavities
}

\author{
C. Behrens ${ }^{1}$, M. Dijkstra ${ }^{1,2,3}$, and J. C. Niemeyer ${ }^{1}$ \\ 1 Institut für Astrophysik, Georg-August Universität Göttingen, Friedrich-Hund-Platz 1, 37077 Göttingen, Germany \\ e-mail: [cbehren;niemeyer] @astro.physik.uni-goettingen.de \\ 2 Max Planck Institute for Astrophysics, Karl-Schwarzschild-Str. 1, 85741 Garching, Germany \\ ${ }^{3}$ Institute of Theoretical Astrophysics, University of Oslo, Postboks 1029, 0858 Oslo, Norway \\ e-mail: mark.dijkstra@astro.uio.no
}

Received 30 October 2013 / Accepted 20 January 2014

\section{ABSTRACT}

\begin{abstract}
We investigate the radiative transfer of $\operatorname{Ly} \alpha$ photons through simplified anisotropic gas distributions, which represent physically motivated extensions of the popular shell models. Our study is motivated by the notion that $(i)$ shell models do not always reproduce observed Ly $\alpha$ spectral line profiles; (ii) (typical) shell models do not allow for the escape of ionizing photons; and (iii) the observation and expectation that winds are more complex, anisotropic phenomena. We examine the influence of inclination on the Ly $\alpha$ spectra, relative fluxes and escape fractions. We find the flux to be enhanced/suppressed by factors up to a few depending on the parameter range of the models, corresponding to a boost in equivalent width of the same amplitude if we neglect dust. In general, lower mean optical depths tend to reduce the impact of anisotropies as is expected. We find a correlation between an observed peak in the occasionally triple-peaked - spectrum at the systemic velocity and the existence of a low optical depth cavity along the line of sight. This can be of importance in the search for ionizing photons leaking from high- redshift galaxies since these photons will also be able to escape through the cavity.
\end{abstract}

Key words. radiative transfer - galaxies: high-redshift

\section{Introduction}

There is ample observational evidence that Ly $\alpha$ photons scatter through outflowing HI gas as they emerge from the interstellar medium of star forming galaxies. In local galaxies, gas kinematics on interstellar scales is one of the key factors that determines whether Ly $\alpha$ photons escape (Kunth et al. 1998; Atek et al. 2008). Moreover, observed Ly $\alpha$ spectral lines in higher redshift $(z \sim 3)$ galaxies have P-Cygni type profiles which consist of a redshifted emission line and a blueshifted absorption trough, which are clear characteristics of the presence of an outflow (e.g. Shapley et al. 2003). The ubiquitous presence of outflows is also revealed by the blueshifted low-ionization metal absorption lines in a large fraction of Lyman break galaxies (LBGs). These same LBGs typically exhibit a Ly $\alpha$ emission line that is redshifted by $\sim 2-3 \times$ the outflow velocity inferred from the absorption line data, and strongly suggest that Ly $\alpha$ photons have been scattered by $\mathrm{H}$ within the outflow (e.g. Steidel et al. 2010).

Modeling cold, outflowing interstellar gas is an extremely difficult task, and practically requires simulations to fully resolve the interstellar medium (see Dijkstra \& Kramer 2012, and references therein). Models of Ly $\alpha$ emitting galaxies have thus far employed a subgrid model of scattering through the outflow, in which the outflow is represented by a spherical shell of $\mathrm{HI}$ gas (see Ahn et al. 2003; Verhamme et al. 2006, for a description of the shell model). This spherical shell is geometrically thin (thickness is 10\% of the radius of the shell), and is parameterized (primarily) by an HI column density, an outflow velocity, and a dust optical depth. It has been demonstrated that these models can provide good fits to observed Ly $\alpha$ line shapes (Verhamme et al. 2008; Schaerer \& Verhamme 2008; Vanzella et al. 2010).
Recently however, near-infrared spectrographs have measured rest-frame optical nebular lines from galaxies at $z \sim 2$ (where it is possible to detect $\mathrm{H} \alpha$ ) and $z \sim 3$ (where it is possible to detect [OIII]). The detection of these nebular lines constrains the systemic velocity of galaxies, and therefore the spectral shift of the Ly $\alpha$ line relative to their systemic velocity (McLinden et al. 2011; Kulas et al. 2012; Hashimoto et al. 2013). Importantly, it has been demonstrated that having both the shift and the spectral line shape poses a challenge to the shell models for a fraction of $\operatorname{Ly} \alpha$ emitting sources, i.e. since the Doppler parameter $b$ derived from this data seems not to fit the observed low-ionization absorption (Kulas et al. 2012), although the exact fraction of emitters problematic for the shell model is still debatable. There is also evidence for the existence of holes in the HI distribution of some Ly $\alpha$ emitters that are only poorly fitted by the shell model (Chonis et al. 2013). Hence, there is increasing observational demand for models that go beyond the shell model. Moreover, from a physical point of view the shell-model is not satisfactory because its simple geometry does not capture the complex structure of the gas in outflows.

Going beyond the shell model is clearly complicated: breaking spherical symmetry causes the predicted spectral line shapes to depend on viewing angle. Moreover, departures from spherical symmetry introduce new parameters that significantly increase the volume of parameter space that can be explored. In this paper, we investigate Ly $\alpha$ transfer through non-spherical models for winds that are still relatively simple \& physically motivated. Examples of models we study include: (i) shells-withholes; (ii) bipolar winds; and (iii) so called cavity models (see Sects. 2.1, 2.3 for details). The first two classes of models approach a spherical shell model when either the hole becomes 
A\&A 563, A77 (2014)

arbitrarily small, or when the anisotropic velocity component of the bipolar model vanishes. Duval et al. (2014) have investigated different modifications of the shell model, namely statistically isotropic, dusty and clumpy shells. These models are in statistical sense still isotropic and have covering factors near 1 , yielding only few lines of sight that are optically thin, while the models discussed here have strong anisotropies with many optically thin lines of sight. Importantly, Duval et al. (2014) and Laursen et al. (2013) have focussed on studying boosts in equivalent width (EW), averaged over all sightlines. Laursen et al. (2013) points out that there can be departures in this boost from the average along individual sightlines, which is the main focus of our work.

A common property of all our models is that there exist sightlines through the wind which contain a very low (or zero) HI-column density. Our wind models thus include possible escape routes for ionizing photons (which clearly do not exist in the shell models). This property of our models is important, and motivated by a scenario in which ionizing photons escape efficiently only along a fraction of sightlines. This picture is motivated by observations of LBGs for which outflowing lowionization absorption line systems, which trace cold $\left(T \sim 10^{4} \mathrm{~K}\right)$ gas, are not always fully covering the UV-emitting regions ${ }^{1}$; For example, Jones et al. (2013) find that the maximum lowionization covering fraction for their sample at $z>2$ is $100 \%$ in only 2 out of 8 galaxies (see also Heckman et al. 2011, who find evidence for a low covering factor of optically thick, neutral gas in a small fraction of lower redshift Lyman break analogues). This covering factor of cold outflowing gas decreases with redshift (Jones et al. 2012, 2013), which may explain that the escape fraction of ionizing photons increases with redshift as has been inferred $^{2}$ independently by observations of the Ly $\alpha$ forest and the UV-LF of drop-out galaxies (e.g. Kuhlen \& Faucher-Giguère 2012; Robertson et al. 2013). Finally, the possibility that ionizing photons escape highly anisotropically naturally explains the apparently bimodal distribution of the inferred escape fraction in star forming galaxies, in which a small fraction of galaxies have a large $f_{\text {esc }}$ and a large fraction practically are consistent with having $f_{\text {esc }}=0$ (Shapley et al. 2006; Nestor et al. 2011; Vanzella et al. 2012).

From a Ly $\alpha$ transfer point-of-view, the existence of low HI-column density channels can have major observational consequences. For non-spherical gas distribution, Ly $\alpha$ generally escapes anisotropically (see Laursen \& Sommer-Larsen 2007; Verhamme et al. 2012; Zheng \& Wallace 2013, and references therein), where the flux can vary by as much as a factor of $>10$ depending on the viewing angle (Verhamme et al. 2012). We expect this anisotropy to be potentially more extreme in models that contain low- $N_{\mathrm{HI}}$ sightlines, which we refer to as holes and/or cavities. These cavities may correspond to regions that have been cleared of gas and dust by feedback processes (see Nestor et al. 2011, 2013, who describe a simple blow-out model).

In this paper we study Ly $\alpha$ transfer through simplified models of the ISM which include outflow-driven cavities. Our models consist of several families. The shell-with-holes and bipolarwind models can easily be connected to the traditional shell models, which makes it easier to interpret our results. The

\footnotetext{
1 A caveat is that the covering factor is less than unity over a range of velocities. Cold gas moving at different velocities can cover different parts of unresolved UV emitting sources, and it remains possible that the covering factor integrated over velocity is 1 .

2 This redshift evolution appears to be required in order to be able to reionize the Universe (Kuhlen \& Faucher-Giguère 2012).
}

cavity models represent a scenario in which gas is outflowing perpendicular to a galactic disk. This paper has two main goals: (i) to investigate the variation in flux for different viewing angles which will give us an estimate for the expected boost in EW with respect to the isotropic case; and (ii) to investigate whether there are robust observational spectral signatures of the $\operatorname{Ly} \alpha$ line for sightlines that go through a cavity.

The outline of this paper is as follows: in Sect. 2 we introduce our models. In Sect. 3.1, we discuss and interpret our results with a focus on individual dust-free realizations of our model families. In Sects. 3.2 and 3.3, we consider the influence of dust and generalize our results with a parameter study.

\section{Our models and parameters}

In order to investigate the variation of fluxes and spectra with viewing angle, we have to break the symmetry of the models by introducing anisotropic velocity and/or density fields. Like the shell model, our models are primarily motivated by situations where galactic winds and other feedback mechanisms drive outflows, blowing out or ionizing neutral gas. We take into account the asymmetry of such winds that are expected to occur particularly in the transverse direction of the plane. This introduces an anisotropy in this direction. We define the inclination as the angle between the $z$-axis and a unit vector towards the observer. To quantify the anisotropy of fluxes and spectra, we define a set of observers, each observing photons within a certain angular bin. Bins are equally spaced in $\cos (\theta)$ so that each observer sees the same area of the unit sphere, meaning that in isotropic cases, all observers should detect a statistically equal number of photons. As usual, we measure the frequency of escaping photons in the dimensionless quantity $x=\Delta v / v_{\mathrm{D}}$, where $\Delta v$ is the physical frequency centered on the $\operatorname{Ly} \alpha$ line center and $\nu_{\mathrm{D}}$ is the Doppler frequency.

\subsection{The bipolar model}

Here, we assume the wind to drive the expanding shell asymmetrically. This deforms the shell over time and displaces it anisotropically. Likewise, the density evolves with inclination, because mass conservation ensures that the mass within a solid angle remains constant. We define the velocity at an angle $\theta$ to be

$\boldsymbol{v}(\boldsymbol{r})=\boldsymbol{e}_{r}\left(v_{\mathrm{b}} \cos (\theta)+v_{\mathrm{c}}\right)$,

where $\boldsymbol{e}_{r}$ is the unit vector from the center to $\boldsymbol{r}, v_{\mathrm{b}}$ is the magnitude of the anisotropic velocity component and $v_{\mathrm{c}}$ is the isotropic component. At a given inclination $\cos \theta$, the shell will be displaced by some distance $s$ from the original position,

$s=\left(v_{\mathrm{b}} \cos (\theta)+v_{\mathrm{c}}\right) t$.

Here, $t$ is some time parameter that controls the evolution of the shell's shape. We caution the reader that for our radiative transfer, we assume a steady state, e.g. $t$ does not evolve during the calculation. $t$ is given in units of the simulation box crossing time, e.g. $s$ becomes the box length for $t=1$. The density within the shell at a given inclination reads

$\rho=\rho_{0} \frac{r_{i}^{2}+r_{i} r_{\mathrm{o}}+r_{\mathrm{o}}^{2}}{3 s^{2}+r_{i}^{2}+r_{i} r_{\mathrm{o}}+r_{\mathrm{o}}^{2}+3 s\left(r_{i}+r_{\mathrm{o}}\right)}$,

where $\rho_{0}$ is some hydrogen density defined such that at $t=0$

$N_{\mathrm{H}}^{\prime}=\rho_{0}\left(r_{\mathrm{o}}-r_{i}\right)$. 
C. Behrens et al.: Beamed Ly $\alpha$ emission through outflow-driven cavities

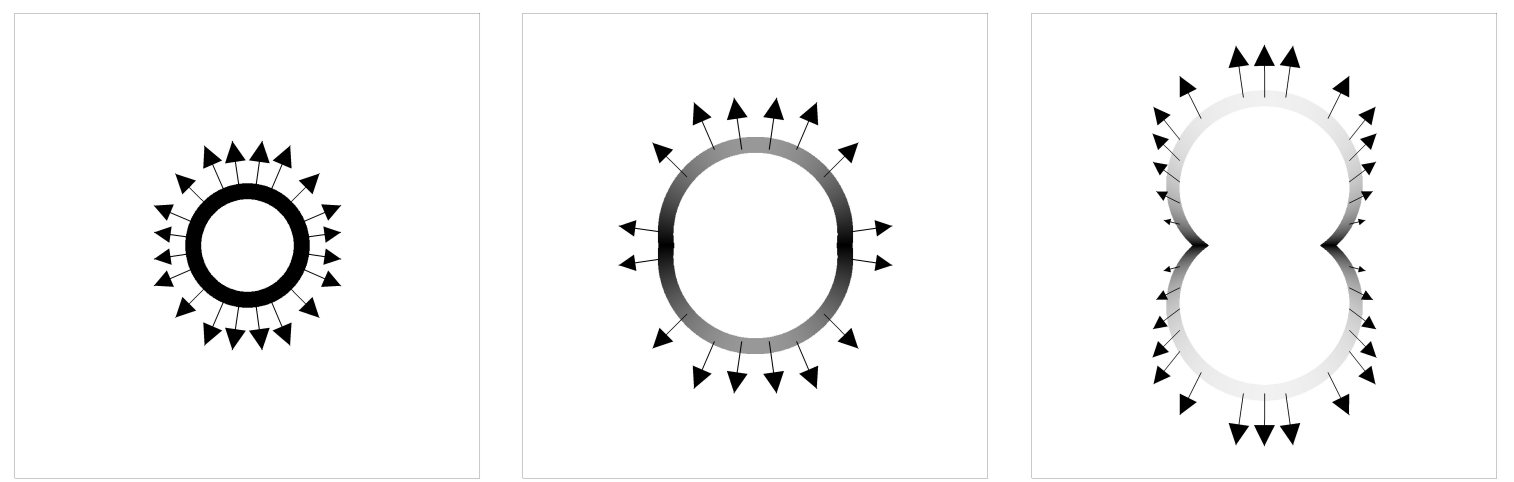

Fig. 1. Illustration of the bipolar model for three different time parameters. Arrows indicate velocity vectors, contours the density distribution. As the time parameter evolves from left to right, the anisotropy in the bulk velocity becomes visible in the density distribution.

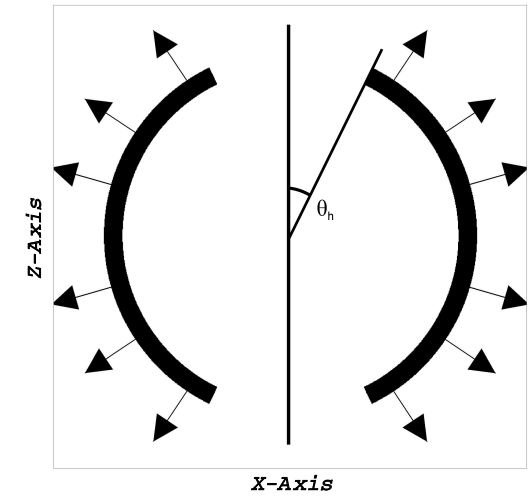

Fig. 2. Illustration of the shell-with-holes model. Arrows indicate velocity vectors, contours the density distribution.

We denote this column density with an additional prime because it is not the effective column density if $t \neq 0$. In Fig. 1, this setup and its dependence on the time parameter is illustrated. In practice, we parametrize $v_{\mathrm{c}}$ and $v_{\mathrm{b}}$ by the sum of both $v_{\text {tot }}$ and the fraction $f_{\mathrm{b}}$ that goes into parametrizing the anisotropic velocity field

$v_{\mathrm{b}}=f_{\mathrm{b}} v_{\text {tot }}$

\subsection{The shell-with-holes model}

Assuming a spherically expanding hydrogen shell around the emitter, in one limiting case the wind emerging from the ISM might just blow off the cap of the spherical shell in the $\pm z$ direction, carving a conical region out of the shell. A cut through the density field of such a setup can be seen in Fig. 2. The solid angle subtended by the conical holes in the shell, $\Omega$, is the only new parameter in this setup and can be also parametrized by the angle $\theta_{\mathrm{H}}$ as indicated in Fig. 2.

\subsection{The cavity model}

In a later stage, the galactic feedback might have completely swept away or ionized the gas along its path, while in the plane, cold gas is still accreted. In an extreme scenario, this leads to a quasi-static situation where a small region along the $\pm z$-axis
Table 1. Summary: model parameters.

\begin{tabular}{lc}
\hline \hline Model & Parameter set \\
\hline Shell & $N_{\mathrm{H}}, v, b$ \\
Bipolar & $N_{\mathrm{H}}^{\prime}, b, v_{\text {tot }}, f, t$ \\
Shell-with-holes & $N_{\mathrm{H}}, v, b, \Omega$ \\
Cavity & $N_{\mathrm{H}}{ }^{\prime \prime}, b$, parameters of the parabolae, $v_{l}$ \\
\hline
\end{tabular}

is essentially evacuated. This model is somewhat similar to the shell-with-holes model, but due to the gaseous torus around the cavity, there is a high probability that photons coming from random angles are scattered so that they are eventually aligned with the $z$-axis. Here, our density profile is described by the intersection of two rotated parabolae, $p_{1}(x)=a_{1}\left(|x|-b_{1}\right)^{2}+c_{1}$ and $p_{2}(x)=a_{2}\left(|x|-b_{2}\right)^{2}+c_{2}$ where $x$ is the perpendicular distance from the $z$-axis. The density at a point at distance $x^{\prime}$ from the $z$-axis and at a height $h$ above the plane is non-zero when $h<p_{1}\left(x^{\prime}\right)$ and $h<p_{2}\left(x^{\prime}\right)$.

We define $r_{\mathrm{c}}$ to be the radius of the object at $z=0$ and set $\frac{N_{\mathrm{H}^{\prime \prime}}}{r_{\mathrm{S}}}=\rho$. We label the column density with two primes because it is not equivalent to the column densities in the models above since the effective column density seen by a photon emitted at an initial angle $\theta_{0}$ will non-trivially depend on this angle due to the shape of the density distribution (see also Fig. 3) Additionally, we add a linear velocity field

$\boldsymbol{v}=\boldsymbol{e}_{z} \frac{z}{z_{\max }} v_{l}$,

which is proportional to the height over the plane and parallel to the $\pm z$-axis. $z_{\max }$ is the $z$-coordinate of the "tip" of the density distribution. This setup is illustrated in Fig. 3.

\section{Results}

\subsection{Flux and spectra for individual models}

In this section, we show results for three individual realizations of our models. If not stated otherwise, we set the Doppler parameter $b$ to $40 \mathrm{~km} \mathrm{~s}^{-1}$ and insert the Ly $\alpha$ photons with a Gaussian distribution around the line center, where the width $\sigma$ is set to $100 \mathrm{~km} \mathrm{~s}^{-1} \cdot 10^{7}$ photons were calculated for every realization to ensure convergence. For the models presented here, we show the column density and initial optical depth (for a photon at line center) as a function of inclination in Fig. 4. 
A\&A 563, A77 (2014)

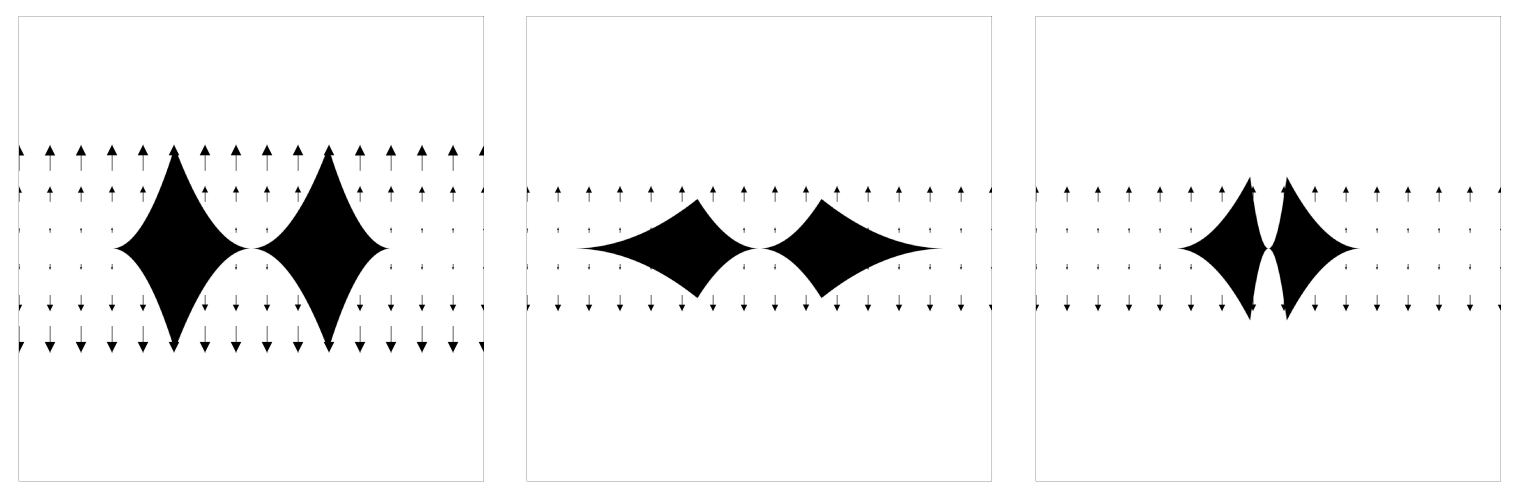

Fig. 3. Illustration of the cavity model with three different parameter sets for the parabolae that define the density distribution. Arrows indicate velocity vectors, contours the density distribution.
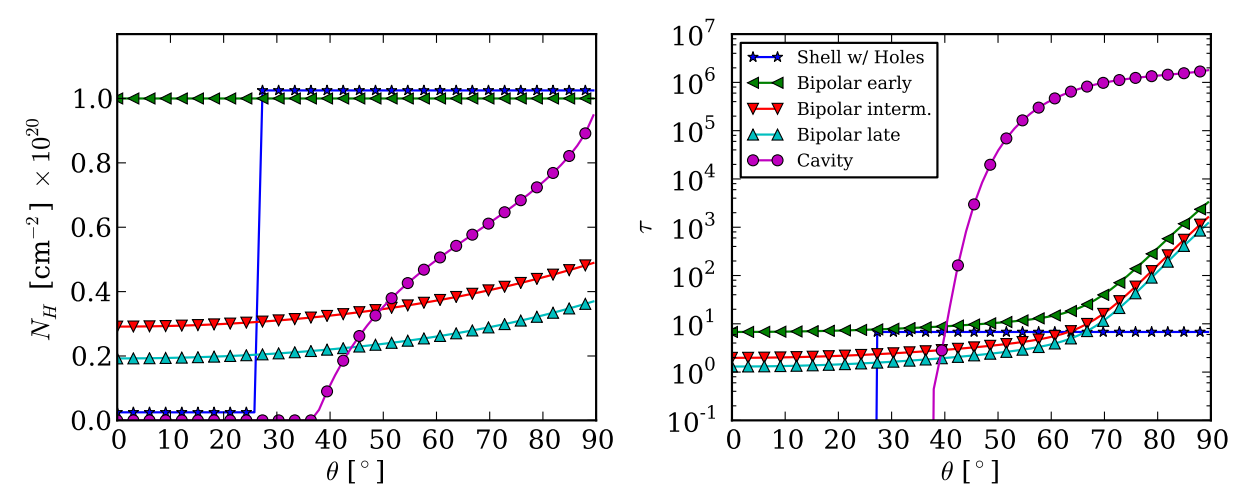

Fig. 4. Neutral hydrogen column density (left) and optical depth (right) of photons starting in the center of the setup with $x=0$ for realizations of different models discussed in Sect. 3.1 as a function of the angle $\theta$ between the line of sight an the the $z$-axis. Note the log-scaling in the right plot.

\subsubsection{Bipolar}

In the upper panel of Fig. 5, we show the flux as a function of viewing angle for a particular case of the bipolar model $\left(v_{\text {tot }}=200 \mathrm{~km} \mathrm{~s}^{-1}, f_{\mathrm{b}}=0.5, N_{\mathrm{H}}^{\prime}=10^{20} \mathrm{~cm}^{-2}, t=0\right)$. Values are given in units of the corresponding isotropic distribution among the observers. Let $F=n / N$ be the relative flux an observer detects, where $n$ is the number of photons received by the observer and $N$ the total number of photons simulated. Further, let $F_{0}=N / n_{\text {bins }}$ be the relative flux assuming isotropy with $n_{\text {bins }}$ the number angular bins. The flux in these bins can be seen as the flux an observer at a specific inclination would obtain. $F / F_{0}$ measures the relative flux relative to the isotropic case. We stress that assuming the object to be dust-free, this quantity is proportional to the $\mathrm{EW}$ of the observed $\mathrm{Ly} \alpha$ line.

Since for the setup in Fig. 5 the time parameter is zero, the density field is equivalent to the isotropic shell model, but the velocity field exhibits an anisotropy, lowering the optical depth along the $z$-axis by a factor of $10^{3}$ with respect to lines of sight within the $x y$-plane. Since the time parameter is zero, we refer to this model as early-bipolar model. As can be seen in the upper panel of Fig. 5 the flux variation is small, about $15 \%$ more photons escape at $\theta \approx 0^{\circ}$ than at $\theta \approx 90^{\circ}$. In the bottom panels of Fig. 5, we show spectra obtained at three different observations angles. Horizontal lines in the top panel show at which angles the spectra where evaluated. For these parameters, the shape of the spectrum does not change significantly with observation angle. The three different lines Fig. 5 show the results for different input spectra with a width of $100 / 40 / 10 \mathrm{~km} \mathrm{~s}^{-1}$ (solid/dotted/dashed lines). As expected, a narrow input spectrum leads to a higher and narrower peak for small observation angles. The flux does not change significantly with respect to the width of the Gaussian.

In Fig. 6, we show the same quantities for a more evolved setup with $t=0.15$ which will we will refer to as the latebipolar case. Here, the flux distribution shows a counterintuitive trend; while flux is suppressed for large observations angles, it is also suppressed for very small angles, leading to a maximum at around $\theta=38^{\circ}$. Detailed investigation shows that this is a geometrical effect from the deformation of the shell. Most parts of the surface of the bipolar shell point towards the $x y$-plane. Photons escaping the shell in these areas will most likely not escape with a small angle $\theta$. Photons that do escape with small angles most probably escape from the poles. This leads to enhanced flux at intermediate angles, because photons escaping at these angles can statistically escape at every point of the surface, while those at small/large angles are confined to certain areas. This could also be described as a "projection effect". Our explanation is further supported by the fact that the effect becomes stronger for a broader input spectrum. The blue part of the Gaussian is subject to scatterings even for photons traversing the shell along the $z$-axis. The broader the spectrum, 
C. Behrens et al.: Beamed Ly $\alpha$ emission through outflow-driven cavities
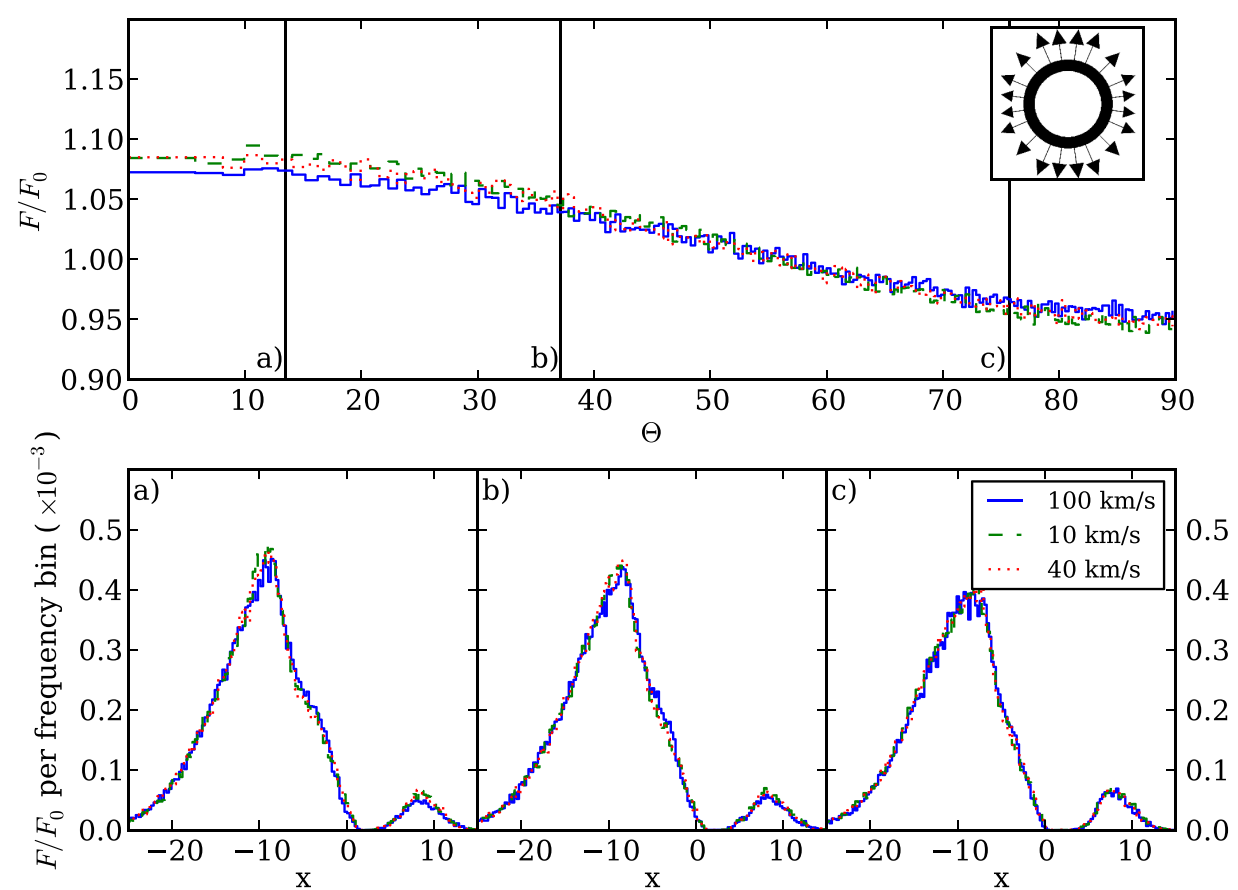

Fig. 5. Top: flux as a function of angle of observation for the early-bipolar model. Units are chosen so that a value of 1 would correspond to the isotropic case. Bottom: spectrum evaluated at three different angular bins (indicated by vertical lines above). Results are shown for input spectra with a width of 100 (blue, solid), 10 (green, dashed) and 40 (red, dotted) $\mathrm{km} \mathrm{s}^{-1}$. Spectra are normalized to the total flux in the angular bin. Model parameters are $N_{\mathrm{H}}^{\prime}=10^{20} \mathrm{~cm}^{-2}, v_{\mathrm{tot}}=200 \mathrm{~km} \mathrm{~s}^{-1}, f_{\mathrm{b}}=0.5, t=0$.
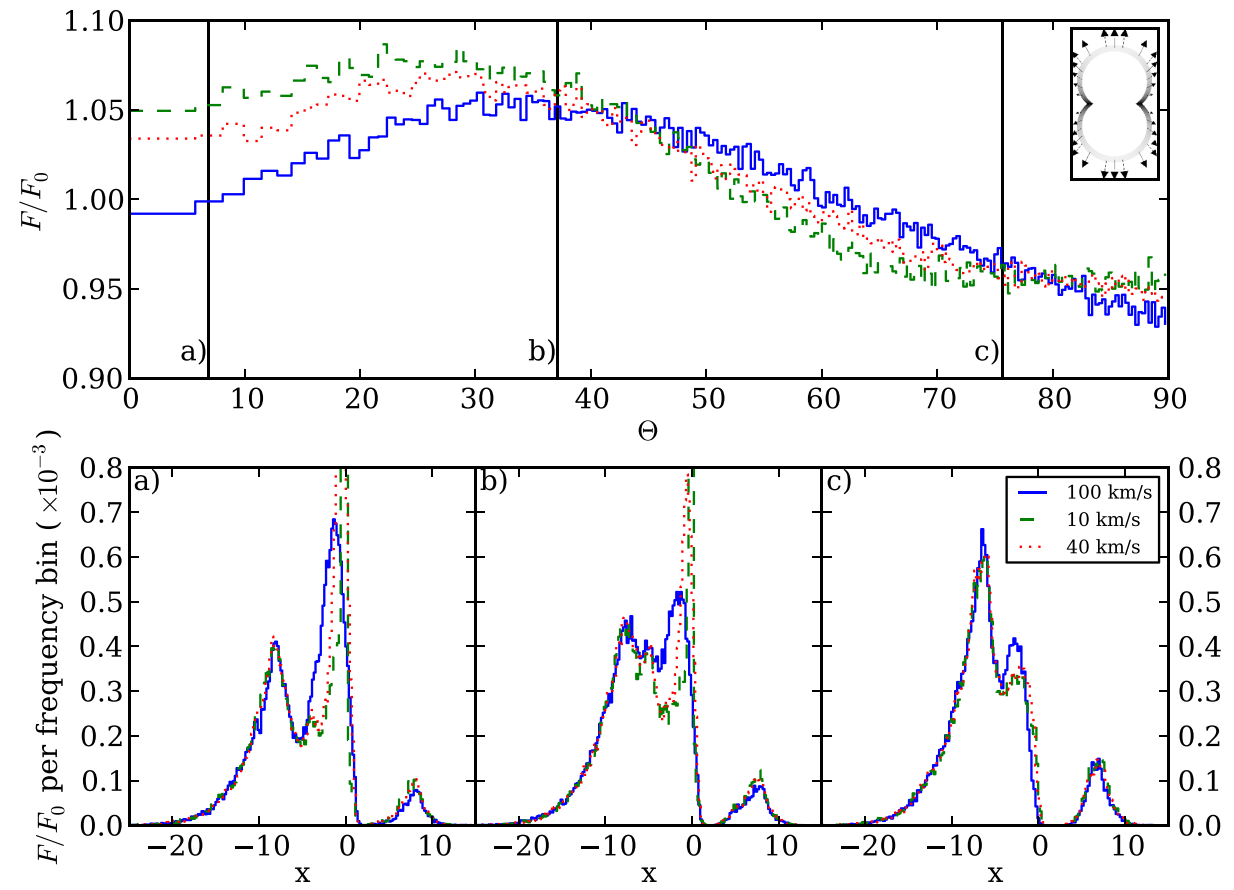

Fig. 6. Same as Fig. 5, but for the late-bipolar model. Model parameters are $N_{\mathrm{H}}^{\prime}=10^{20} \mathrm{~cm}^{-2}, v_{\text {tot }}=200 \mathrm{~km} \mathrm{~s}^{-1}, f_{\mathrm{b}}=0.5, t=0.15$. 
A\&A 563, A77 (2014)
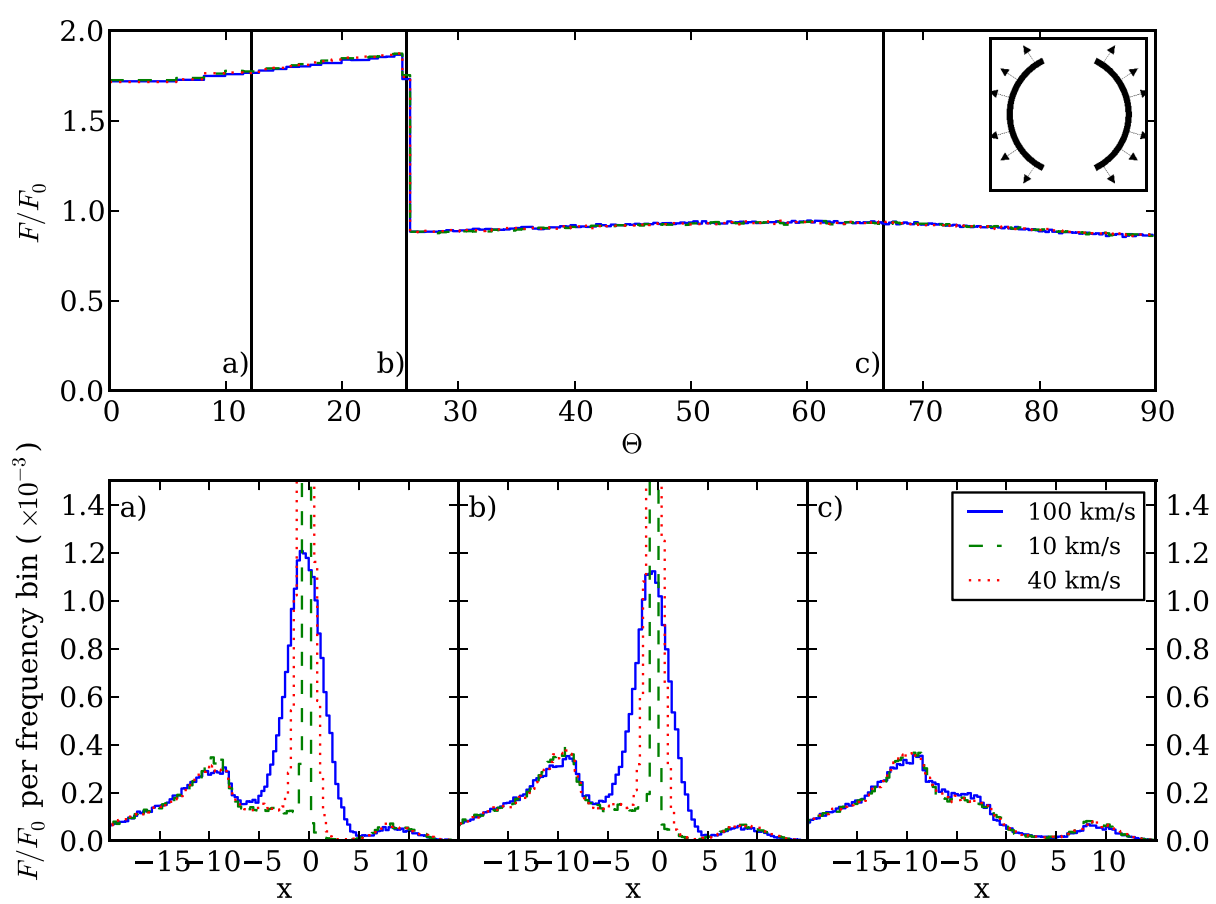

Fig. 7. Same as Fig. 5, but for the shell-with-holes model. Model parameters are $v=200 \mathrm{~km} \mathrm{~s}^{-1}, N_{\mathrm{H}}=10^{20}$ and $\Omega=10 \%$, meaning that $10 \%$ of the shell is subtended by the holes.

the more blue photons are scattered at the poles, redistributing their orientation and further enhancing measured flux at intermediate angles.

The spectra shown in the bottom panels in Fig. 6 again show a peak at $x=0$ for observers looking down the $z$-axis (bottom left panel). For an input spectrum with a width of $100 \mathrm{~km} \mathrm{~s}^{-1}$, it is slightly shifted. This can be attributed to the suppression of the blue part of the input spectrum due to scatterings in the outflowing material. For smaller widths, the peak is clearly centered at $x=0$ again. For observers around $\theta=40^{\circ}$, the amplitude of the peak is lower. If we go to larger angles, the central peak vanishes and the red peak visible in all of the three spectra becomes dominant.

\subsubsection{Shell-with-holes}

The dependence of flux and spectra for one particular case $(v=$ $200 \mathrm{~km} \mathrm{~s}^{-1}, N_{\mathrm{H}}=10^{20} \mathrm{~cm}^{-2}, \Omega=10 \%$ ) of the shell-with-holes model is shown in the upper panel of Fig. 7.

As can be seen in Fig. 7, the observed flux decreases abruptly at an angle of $\approx 25^{\circ}$, which reflects the angular size of the holes in the shell. In the lower panels of Fig. 7, we show spectra of the photons in three different angular bins. Their positions are indicated by vertical lines in the upper panel. The bottom left spectrum shows the spectrum for an observer that directly looks "down the hole". As expected, one can clearly identify the unprocessed, Gaussian input centered around $x=0$. The spectrum in the bottom center shows a spectrum directly before the transition; the transition itself occurs so fast that we do not see it occuring in one of the bins. The bottom right spectrum shows an observer at an angle of $\approx 67^{\circ}$. Here, the peak at line center is clearly gone. Instead, the spectrum more resembles the original shell case. We note that observers looking "down the hole" see a $\operatorname{Ly} \alpha$ line that is not shifted. In this particular setup, observing face-on will boost the flux and therefore the EW by a factor of roughly 1.7 (assuming no scattering of the continuum emission here). It is interesting that apart from the sharp transition at the hole's half opening angle, the spectrum does not evolve significantly. This can be seen by comparing the center bottom and right bottom spectrum. We stress that both the flux and the spectrum change very rapidly and are nearly constant before and after the transition. Beyond the transition, the spectrum resembles the normal shell case. Before the transition, it consists of two components, the initial unscattered spectrum and another contribution from a shell-like spectrum. The latter contribution comes from photons that merely scatter into the line of sight. This implies that for decreasing hole radius the spectrum asymptotes toward the original shell case.

\subsubsection{Cavity model}

In the upper panel of Fig. 8 the flux distribution for one realization of the cavity model is shown. The model parameters here are $N_{\mathrm{H}}^{\prime \prime}=10^{20} \mathrm{~cm}^{-2}, v_{l}=200 \mathrm{~km} \mathrm{~s}^{-1}$, we use the configuration illustrated in the left plot of Fig. 3. Similar to the shell-with-holes model, we get a sharp transition at some "effective opening angle". Here, flux is suppressed up to a factor of 6 for observers looking edge-on, which as discussed above would also correspond to a boost/suppression of the EW by a factor of 3 with respect to what one expects in an isotropic environment. When looking at the three spectra shown at the bottom of Fig. 8, one notices that in contrast to the shell with holes case, the spectral 
C. Behrens et al.: Beamed Ly $\alpha$ emission through outflow-driven cavities

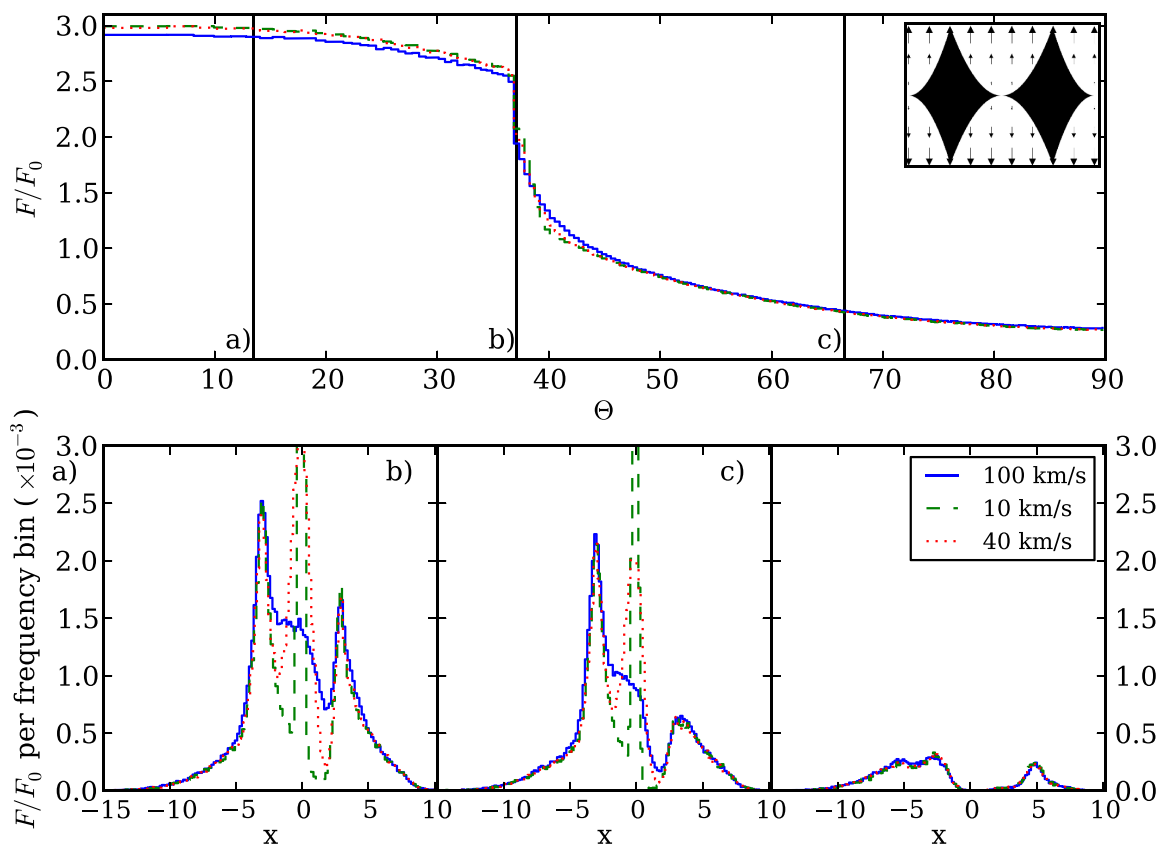

Fig. 8. Same as Fig. 5, but for the cavity model. Model parameters are $N_{\mathrm{H}}^{\prime \prime}=10^{20} \mathrm{~cm}^{-2}, v_{l}=200 \mathrm{~km} \mathrm{~s}^{-1}$.

properties do not change abruptly. The amplitude of the peaks is reduced with viewing angle, and the overall amplitude shrinks, but the input spectrum is not visible anymore at least for the fiducial case with an width of the input spectrum of $100 \mathrm{~km} \mathrm{~s}^{-1}$ (solid). The reason for this different behaviour is that while observers near face-on position still see a significant fraction of photons that directly traversed the cavity, the amount of photons that merely scattered into the line of sight is much bigger. Looking closely at the left and center spectra, one can see the right shoulder of the Gaussian input spectrum. This modified Gaussian is gone far away from the transition, which leads to negligible flux around $x=0$, as can be seen in the right bottom plot of Fig. 8. For narrower input line, we recover the input spectrum again. We conclude that while we get a large flux suppression for this setup, observers could only distinguish from the spectra alone whether or not they are looking down the hole or not if the initial line is much narrower than $100 \mathrm{~km} \mathrm{~s}^{-1}$. The maximum amplitude of a broad Gaussian is not bright enough to be distinguished from the rest of the spectrum.

We note that in contrast to the late bipolar model, we do not see a suppression of the flux at small angles due to geometrical effects although the density field in this case is clearly deformed as well. We attribute this to the fact that the differences in optical depth among different lines of sight are much larger than in the discussed bipolar setup. In contrast to the shell-with-holes model, in this case the transition in the spectral shape and the flux is not as rapid, but more smoothly. This is can be explained by considering the smooth transition in column density and optical depth as shown in Fig. 4, as well as by the fact that the escape mechanism for the cavity model is different (see below).

\subsection{The influence of dust}

While not being the main focus of this paper, the influence of dust on the aforementioned phenomena is also of interest.
Therefore, we reran the individual cases presented in the previous sections with varying dust content. We introduced dust to be proportional to hydrogen content, following the implementation given in Verhamme et al. (2006) with an albedo of 0.5 and an isotropic phase function in case of scattering on dust. We quantify the dust content in terms of the additional optical depth $\tau_{\mathrm{D}}$. Similar to the hydrogen column density, $\tau_{\mathrm{D}}$ is the depth through the shell for the shell-with-holes model, the optical depth at $t=0$ for the bipolar models and the optical depth in the plane for the cavity model. The results are shown in Figs. 9-12. All runs were perfomed using an input spectrum of width $100 \mathrm{~km} \mathrm{~s}^{-1}$, and assuming $b=40 \mathrm{~km} \mathrm{~s}^{-1}$.

As expected, the dependence of the flux distribution on inclination in general becomes larger for higher dust content. For the shell-with-holes case in Fig. 11, we see a boost in the flux anisotropy by a factor of 3 with respect to the dust-free case: photons not escaping through the holes are subject to destruction by dust, so relatively the flux through the holes increases. The same accounts for both the bipolar cases in Figs. 9 and 10, although the effect is less strong. Interestingly, dust does not strongly influence the cavity model as shown in Fig. 12 if one compares to the shell-with-holes case. Surprisingly, the $\tau_{\mathrm{D}}=2$ just increases the flux boost by a factor $\approx 20 \%$. Analysis of the spatial distribution with regard to the point of last scattering of escaping photons show that most of the photons bounce of the inner trough of the density distribution without penetrating deeply, so most photons do not experience the dust opacity. This escape mechanism also partly explains the smooth transition of the spectra/flux distribution mentioned before.

\subsection{Parameter study: peak position versus observation angle}

To examine the correlation of a peak at the systemic velocity (at $x=0$ ) with low inclination, we ran a suite of models with 
A\&A 563, A77 (2014)
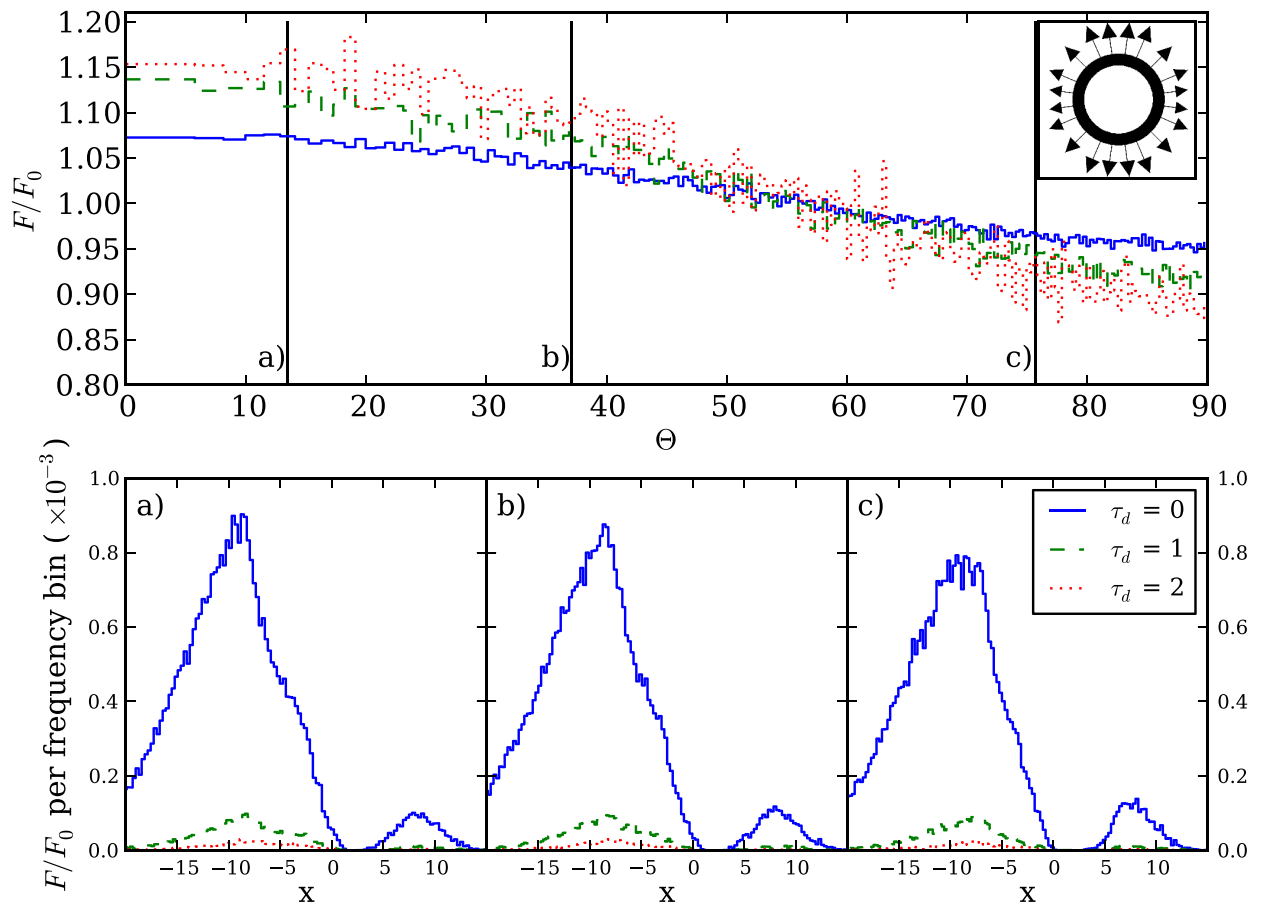

Fig. 9. Same as Fig. 5 (early-bipolar model), but for three different optical depths in dust: $\tau_{\mathrm{D}}=0$ (blue, solid), 1(green, dashed), 2(red, dotted). Model parameters are $N_{\mathrm{H}}^{\prime}=10^{20} \mathrm{~cm}^{-2}, v_{\text {tot }}=200 \mathrm{~km} \mathrm{~s}^{-1}, f=0.5, t=0$.
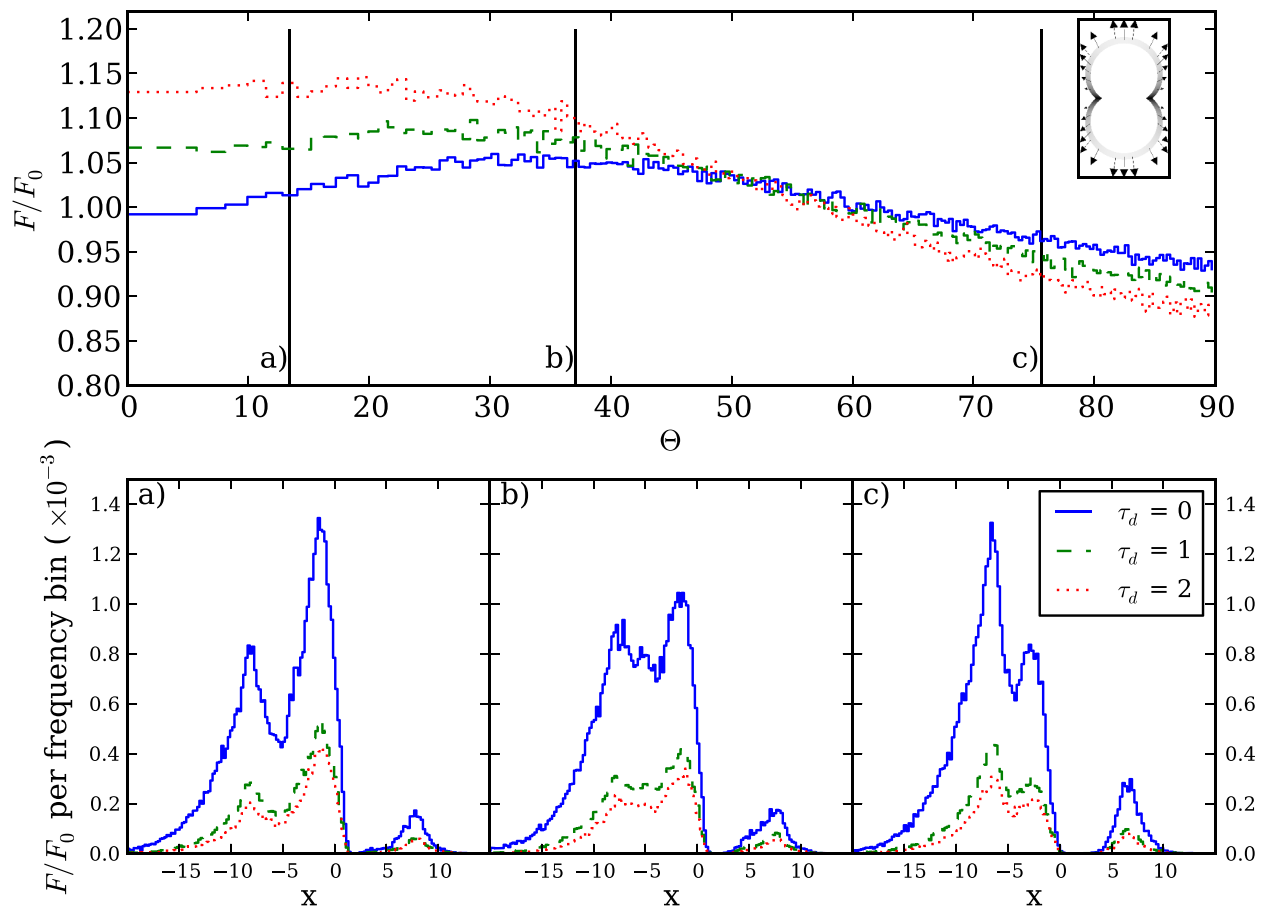

Fig. 10. Same as Fig. 9, but for the late-bipolar model. Model parameters are $N_{\mathrm{H}}^{\prime}=10^{20} \mathrm{~cm}^{-2}, v_{\mathrm{tot}}=200 \mathrm{~km} \mathrm{~s}^{-1}, f=0.5, t=0.15$ 
C. Behrens et al.: Beamed Ly $\alpha$ emission through outflow-driven cavities
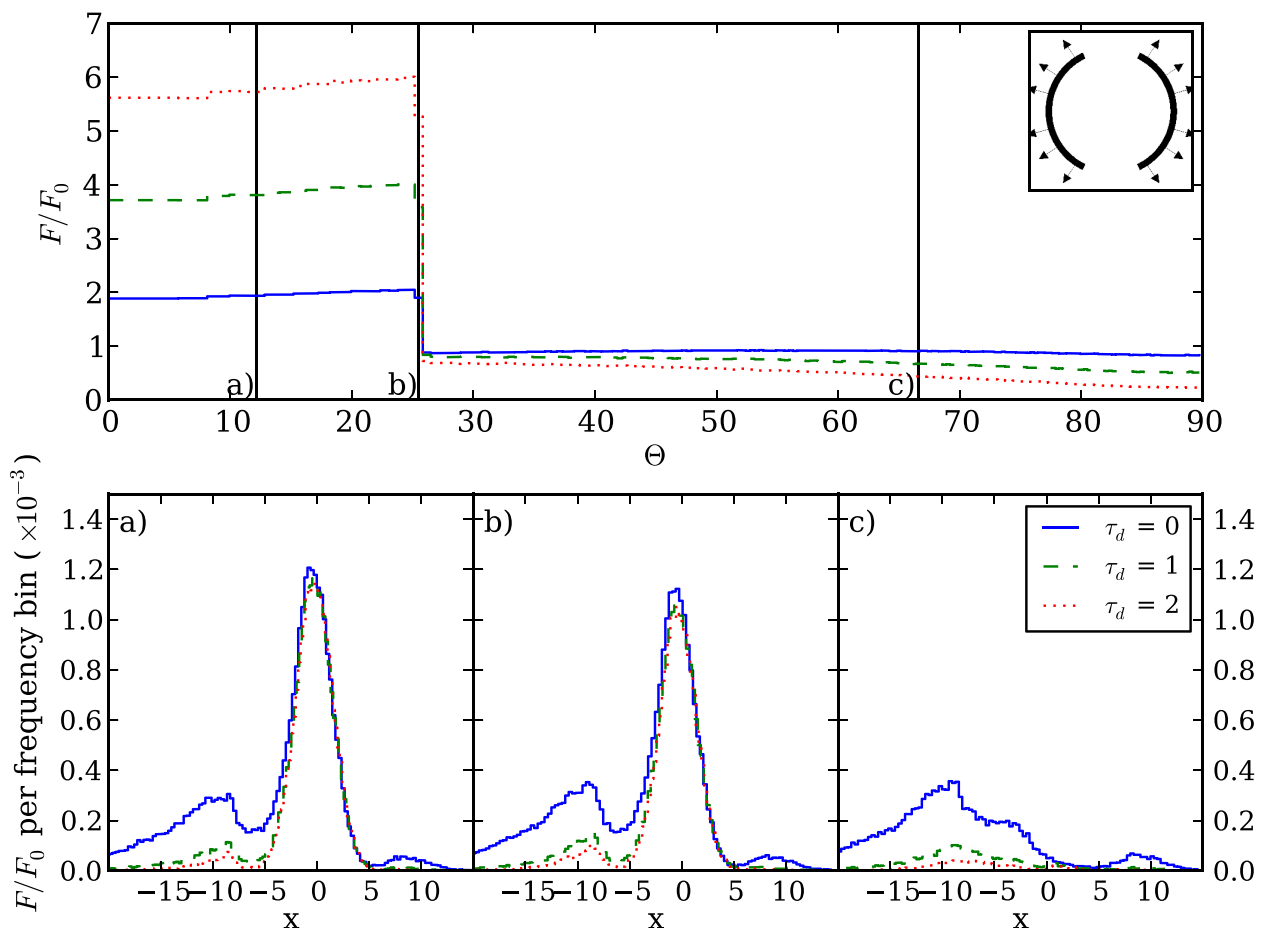

Fig. 11. Same as Fig. 9, but for the shell-with-holes model. Model parameters are $v=200 \mathrm{~km} \mathrm{~s}^{-1}, N_{\mathrm{H}}=10^{20}$ and $\Omega=10 \%$.
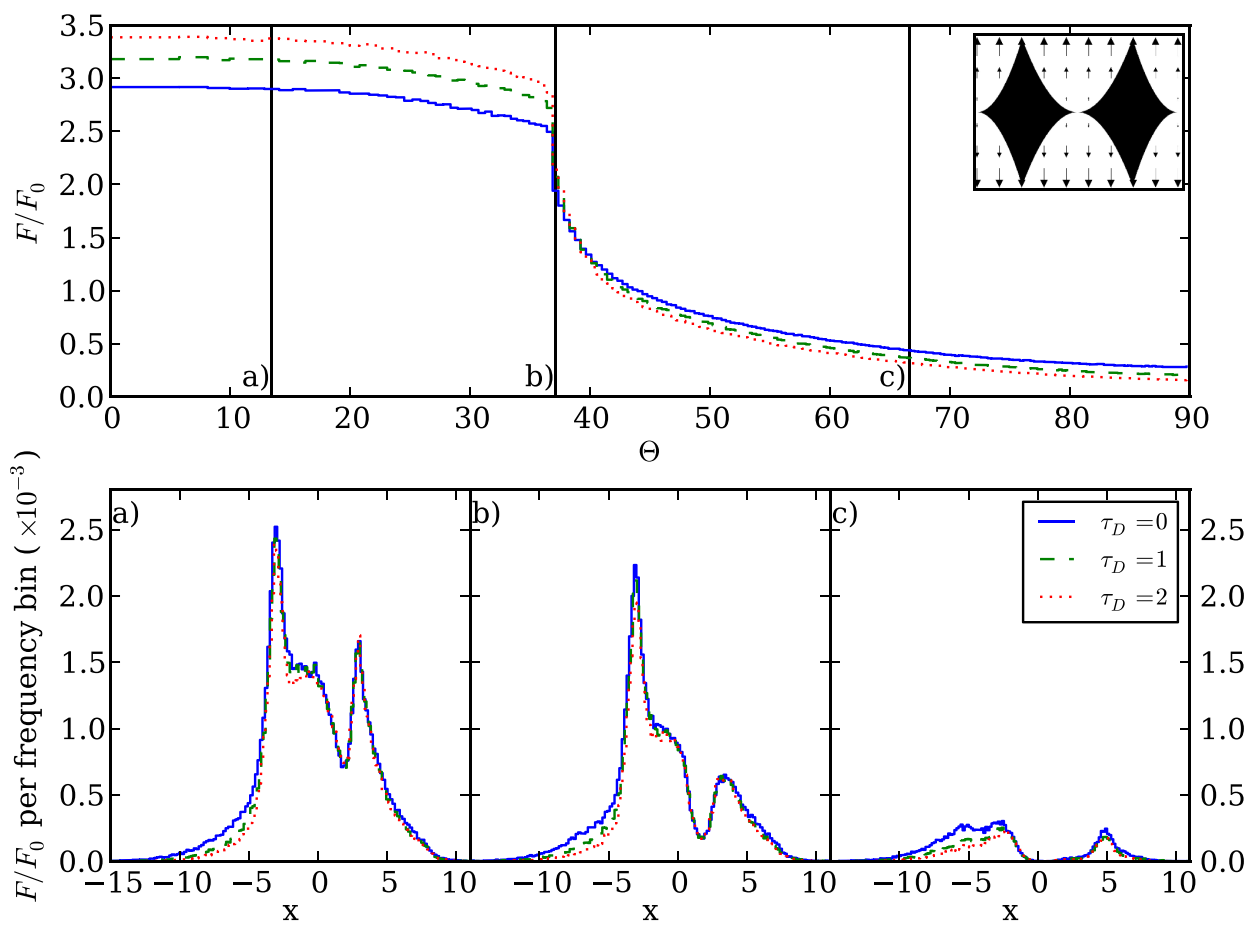

Fig. 12. Same as Fig. 11, but for the cavity model. Model parameters are $N_{\mathrm{H}}^{\prime \prime}=10^{20} \mathrm{~cm}^{-2}, v_{l}=200 \mathrm{~km} \mathrm{~s}^{-1}$. 
A\&A 563, A77 (2014)

Table 2. Summary: explored parameters.

\begin{tabular}{lc}
\hline \hline Model & Parameter set \\
\hline Bipolar & $N_{\mathrm{H}}^{\prime}=\left[10^{19}, 10^{20}, 10^{21} \mathrm{~cm}^{-2}\right], v_{\text {tot }}=\left[100,200,300 \mathrm{~km} \mathrm{~s}^{-1}\right], f=[0.25,0.5,0.75,1.0], t=[0,0.05,0.1,0.2]$ \\
Shell-with-holes & $N_{\mathrm{H}}=\left[10^{19}, 10^{20}, 10^{21} \mathrm{~cm}^{-2}\right], v=\left[100,200,300 \mathrm{~km} \mathrm{~s}^{-1}\right], \Omega=[5 \%, 10 \%, 25 \%, 30 \%]$ \\
Cavity & $N_{\mathrm{H}}^{\prime \prime}=\left[10^{19}, 10^{20}, 10^{21} \mathrm{~cm}^{-2}\right], v_{l}=\left[0,100,200,300 \mathrm{~km} \mathrm{~s}^{-1}\right]$ \\
\hline
\end{tabular}
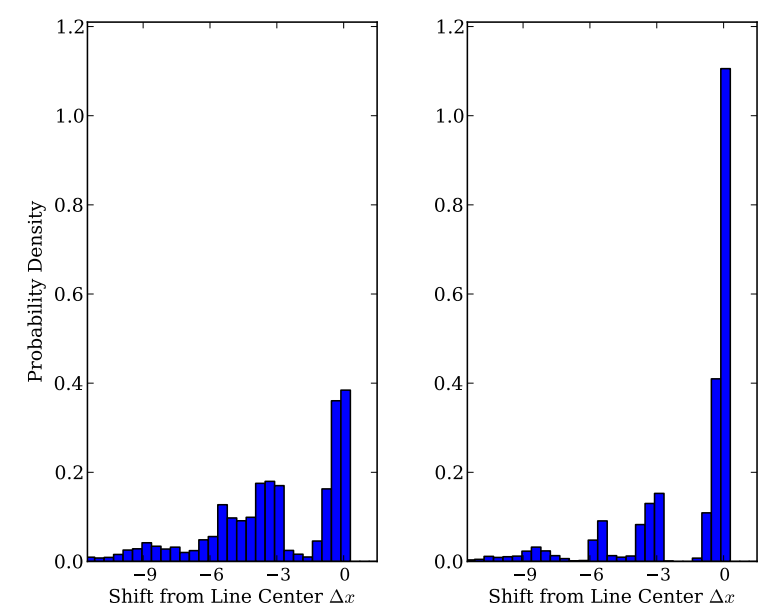

Fig. 13. Distribution of the spectral peak for our parameter study, see text for details. Left: distribution of peak position for observers with $\theta>42^{\circ}$. Right: distribution for observers with $\theta<42^{\circ}$.

different parameters. We set the width of the input spectrum to $40 \mathrm{~km} \mathrm{~s}^{-1}$ here. The parameter range for the three different model families is shown in Table 2. In total, we ran 272 models, all without dust. Since certain parameter combinations lead to weak anisotropies in optical depth and column density, we restricted our analysis to those for which either the flux changed by at least $10 \%$ with respect to the observation angle and/or for which the highest peak in the spectrum moves at least 3 units in frequency depending on observation angle. We stress that by these conditions, we do not select only those models that already show the aforementioned correlation, but all those models that show anisotropic spectra and/or fluxes in general. We end up with a total of 179 models. In Fig. 13, we show the distribution of the peak position for those models. In the left panel, the distribution is shown for observers at angular positions $\theta>42^{\circ}$, while in the right panel, we show the distribution for observers within $\theta<42^{\circ}$. For the latter, there is a strong peak at $x \approx 0$. While for observers above $42^{\circ}$ there is a peak at the systemic velocity as well, it is much smaller than for observers below $42^{\circ}$. Within the scanned region of parameter space, we therefore find a correlation between peak shift and inclination. For low inclination, the probability to see a peak at $x \approx 0$ is enhanced by a factor of a few. This trend is consistent in all our three model families, but stronger for the shell-with-holes and cavity models. This suggests that a $\operatorname{Ly} \alpha$ spectrum with a peak at $\Delta x \sim 0$ is more likely (than random) to be associated with a sightline passing through a hole or cavity. We stress that due to the finite spectral resolution of observations, the peaks can be washed out. In this case, we don't expect a distinct peak at systemic velocity, but a flux excess near the systemic velocity.

\section{Discussion and conclusions}

We performed Monte-Carlo radiative transfer simulations for the Ly $\alpha$ emission in three different families of models that are all inspired by galactic winds and expanding $\mathrm{H}$ shells. In contrast to the shell models (e.g Verhamme et al. 2006), we have added anisotropic velocity and density components. While the bipolar and shell with holes models are natural extensions to the isotropic shell models, the cavity models are designed to be more extreme with large anisotropies (and reminiscent of a wind breaking out of a galactic disk).

We find that the anisotropies in our models lead to an anisotropic escape of photons which is visible in the spectral properties as well as in the flux as obtained for observers at different angular positions. We generally find that the viewing angle-dependence of the predicted spectrum is quite complicated, with the flux in different peaks in the spectrum changing in some cases (see e.g. Fig. 10), but not in others (see e.g. Fig. 9). Additionally, we find that the inclination effect due to anisotropic density and velocity fields can be partly counteracted by geometrical effects, e.g. for continously deformed shells like in the bipolar late-type cases.

In spite of these complications there are several results to take away from our analysis:

- In terms of the EW, we find boosts of up to $\sim 10 \%$ for the bipolar model, boosts of $\sim 2$ for the shell-with-holes model and a factor of $\sim 3$ for the cavity model. The inclusion of dust enhances the boost for the bipolar [shell-with-holes] model to $\sim 15 \%$ [a factor of $\sim 5.5$ ]. For the cavity model, the inclusion of dust increases the boost to about 3.5.

- For all of the discussed models, we find a range of parameters for which there is a clear correlation between an observed peak at a velocity shift of zero and an inclination close to zero. This is intuitive, since when looking down a cavity, we expect to see the unaltered spectrum of the emitting region, not strongly affected by scatterings in intervening gas. This can be of practical importance when searching for ionizing flux leaking out of high-redshift galaxies, because the existence of such cavities will also allow ionizing photons to escape the galaxy. Our models suggest that there is an increased probability to find ionizing photons leaking from Ly $\alpha$ emitters for which the $\operatorname{Ly} \alpha$ spectrum exhibitis a peak at systemic velocity. Due to the finite spectral resolution of observations, this peak might be washed out and show as flux excess near the systemic velocity. It is interesting to note that this peak corresponds to the third peak in some of our bipolar and shell-with-holes models. This result holds up even when the total change in flux is small; for the bipolar models, we see the clear signature of such a peak even though the total flux variation is as small as $10 \%$.

Having such an observational diagnostic for sightlines going through low $\mathrm{H}$ column densities is important, as it may provide a new way to search for so-called "Lyman continuum leakers": the search for these galaxies has been highly inefficient as it requires deep spectroscopic observations of 
C. Behrens et al.: Beamed Ly $\alpha$ emission through outflow-driven cavities

galaxies, for most of which no ionizing flux is detected. Thus far, only a handful of these galaxies have been discovered, and consequently the observational constraints on the escape of ionizing photons from star forming galaxies are weak at best. Since this escape fraction plays a key role in the reionization process, it is important to find observational signatures that may help us constrain this quantity (see also Nakajima \& Ouchi 2013 and Jaskot \& Oey 2013 who show that galaxies with high OIII/OII line ratios are more likely to be sources that leak ionizing photons).

- Especially for the cavity models, the observability of the peak at systemic velocity depends strongly on the width of the input line since there are many photons near line center that are scattered into the line of sight of observers that look down the cavity. Moreover, our simulations have infinite spectral resolution. In contrast, the finite spectral resolution of real data washes out the detailed structure of the $\operatorname{Ly} \alpha$ lines. Both points suggest that looking down a low $\mathrm{H}$ sightline does not necessarily give rise to a detectable peak in the Ly $\alpha$ spectrum at $x=0$. However, our analysis does suggest that if such a line is detected, then it may be indicative of having a low $\mathrm{H}$ column density sightline to the galaxy.

- Dust in general enhances the inclination effect, but the influence differs by an order of magnitude or more between our model families.

A preprint by Zheng \& Wallace (2013) appeared recently which also focussed on the escape of Ly $\alpha$ photons from anisotropic gas distributions. While our models share some similarities with theirs, the comparison between their spherical and our shell model extensions are not straightforward since the different geometries have strong effects on the flux distribution. If we quantify the anisotropies by the maximum deviation of the line center optical depth along lines of sight with respect to the mean, as suggested by Zheng \& Wallace (2013), then we find much smaller amplitudes of the inclination effect in comparison to their solid sphere models. For example, in one of our bipolar models, the optical depths along different lines of sight might differ by a factor of $10^{3}$, while the flux does change only by $10 \%$. In Zheng \& Wallace (2013), a difference of a factor of 2 in the optical depth can lead to an enhancement in flux as large as a factor of 2 . We have confirmed the same enhancement in some of the models of Zheng \& Wallace (2013) with our code. This suggests that the deviations in the optical depth do not translate directly into an estimate of the flux change for different geometries.

Acknowledgements. M.D. thanks the the Goettingen Academy of Sciences and Humanities for awarding him a Gauss-Professorship, and the kind hospitality of the Institute for Astrophysics in Goettingen.

\section{References}

Ahn, S.-H., Lee, H.-W., \& Lee, H. M. 2003, MNRAS, 340, 863 Atek, H., Kunth, D., Hayes, M., Östlin, G., \& Mas-Hesse, J. M. 2008, A\&A 488, 491

Chonis, T. S., Blanc, G. A., Hill, et al. 2013, ApJ, 775, 99

Dijkstra, M., \& Kramer, R. 2012, MNRAS, 424, 1672

Duval, F., Shaerer, D., Östlin, G., \& Laursen, P. 2014, A\&A, 532, A52

Hashimoto, T., Ouchi, M., Shimasaku, K., et al. 2013, ApJ, 765, 70

Heckman, T. M., Borthakur, S., Overzier, R., et al. 2011, ApJ, 730, 5

Jaskot, A. E., \& Oey, M.S. 2013, ApJ, 766, 91

Jones, T., Stark, D. P., \& Ellis, R. S. 2012, ApJ, 751, 51

Jones, T., Ellis, R. S., Schenker, M. A., \& Stark, D. P. 2013, ApJ, 779, 52

Kuhlen, M., \& Faucher-Giguère, C.-A. 2012, MNRAS, 423, 862

Kulas, K. R., Shapley, A. E., Kollmeier, J. A., et al. 2012, ApJ, 745, 33

Kunth, D., Mas-Hesse, J. M., Terlevich, E., et al. 1998, A\&A, 334, 11

Laursen, P., \& Sommer-Larsen, J. 2007, ApJ, 657, L69

Laursen, P., Duval, F. \& Ostlin, G. 2013, ApJ,766, 124

McLinden, E. M., Finkelstein, S. L., Rhoads, J. E., et al. 2011, ApJ, 730 136

Nakajima, K., \& Ouchi, M. 2013, MNRAS, submitted [arXiv: 1309. 0207] Nestor, D. B., Shapley, A. E., Steidel, C. C., \& Siana, B. 2011, ApJ, 736, 18 Nestor, D. B., Shapley, A. E., Kornei, K. A., Steidel, C. C., \& Siana, B. 2013, ApJ, 765, 47

Robertson, B. E., Furlanetto, S. R., Schneider, E., et al. 2013, ApJ, 768, 71

Schaerer, D., \& Verhamme, A. 2008, A\&A, 480, 369

Shapley, A. E., Steidel, C. C., Pettini, M., \& Adelberger, K. L. 2003, ApJ, 588 65

Shapley, A. E., Steidel, C. C., Pettini, M., Adelberger, K. L., \& Erb, D. K. 2006 ApJ, 651, 688

Steidel, C. C., Erb, D. K., Shapley, A. E., et al. 2010, ApJ, 717, 289

Vanzella, E., Grazian, A., Hayes, M., et al. 2010, A\&A, 513, A20

Vanzella, E., Guo, Y., Giavalisco, M., et al. 2012, ApJ, 751, 70

Verhamme, A., Schaerer, D., \& Maselli, A. 2006, A\&A, 460, 397

Verhamme, A., Schaerer, D., Atek, H., \& Tapken, C. 2008, A\&A, 491, 89

Verhamme, A., Dubois, Y., Blaizot, J., et al. 2012, A\&A, 546, A111

Zheng, Z., \& Wallace, J. 2013, ApJ, submitted [arXiv: 1308.1405] 
4.3 Inclination Dependence of Lyman- $\alpha$ Properties in a Turbulent Disk Galaxy 


\title{
Inclination dependence of Lyman- $\alpha$ properties in a turbulent disk galaxy
}

\author{
C. Behrens and H. Braun
}

\author{
Institut für Astrophysik, Georg-August Universität Göttingen, Friedrich-Hund-Platz 1, D-37077 Göttingen \\ e-mail: cbehren@astro.physik.uni-goettingen.de/hbraun@astro.physik.uni-goettingen.de \\ Accepted in A\&A, October 2014
}

\section{ABSTRACT}

We present simulations of Lyman- $\alpha$ radiation transfer in an isolated disk galaxy with a turbulence subgrid model, multiphase interstellar medium and detailed star formation modeling. We investigate the influence of inclination on the observed Ly $\alpha$ properties for different snapshots. The $\operatorname{Ly} \alpha$ spectrum, equivalent width distribution, and escape fractions vary significantly with the detailed morphology of the disk, leading to variations from one snapshot to another. In particular, we find that supernova-driven cavities near star-forming regions in the simulation can dominate the transmitted Ly $\alpha$ fraction, suggesting a variability of Lyman- $\alpha$ emitters on the timescales of the star formation activity.

Key words. High-redshift Galaxies - Radiative Transfer

\section{Introduction}

Galaxies detected by their strong Lyman- $\alpha$ (Ly $\alpha)$ emission called Ly $\alpha$ emitters (LAEs), have become an important too in understanding cosmology at intermediate-to-high redshifts $(z>1.5)$, such as for the large-scale matter distribution (Hill et al., 2008; Adams et al., 2011) or the epoch of reionization via the connection of LAEs and Lyman-continuum leakers (Behrens et al., 2014; Verhamme et al., 2014; Dijkstra, 2014). The physical type(s) of objects classified as LAEs and the connection of the observed line profiles to the physical properties of the emitters are still a matter of debate, though progress has been made in recent years (e.g., Ahn et al., 2003; Verhamme et al., 2006; Dijkstra \& White, 2010). In particular, scattering on neutral gas shells around HII bubbles and attenuation by the intergalactic medium (IGM) suppressing the blue part of the spectrum can explain many features of the observed spectra, such as their asymmetry.

In some recent publications, work has been focused on understanding how anisotropies of LAEs in terms of column densities and velocity fields affect observed Ly $\alpha$ properties with respect to the observer's position, mainly for simplified models of LAEs (Zheng \& Wallace, 2013; Laursen et al., 2013; Behrens et al., 2014; Verhamme et al., 2014; Gronke \& Dijkstra, 2014; Duval et al., 2014). For quantifying the inclination dependence of Ly $\alpha$ transmission, more realistic models of LAEs are needed, for example, for estimating how large-scale surveys are affected by a possible alignment of galaxies with the large-scale structure in combination with inclination effects (Hirata, 2009) or other correlations with the large-scale structure (Zheng et al., 2010, 2011; Behrens \& Niemeyer, 2013). While a number of studies of the Ly $\alpha$ transport with more realistic LAEs in cosmological contexts exist (e.g., Tasitsiomi, 2006; Laursen et al., 2009; Yajima et al., 2012; Barnes et al., 2011; Faucher-Giguere et al., 2010) only the work by Verhamme et al. (2012) (hereafter: VDB12) investigates the effects of directional dependence on the basis of high-resolution, dusty, isolated disk simulations in detail. Their work includes radiative transfer calculations in the resolved interstellar medium (ISM) as well as in the transfer of continuum photons for estimating the Ly $\alpha$ equivalent width (EW). VDB12 find the inclination effect to be strong, leading to variations in the observed EW from -5 to $90 \AA$ for edge-on/face-on observers with considerable scatter.

In this paper, we extend our previous work on simplified models that were motivated by the existence of optically-thin outflows in observed galaxies to a more realistic setup and present a new study on the radiative transfer of Ly $\alpha$ and continuum photons in an isolated disk galaxy with an advanced model of star formation, turbulence and feedback. In particular, we use snapshots of our model galaxy spanning $1 \mathrm{Gyr}$ of its evolution to quantify differences in Ly $\alpha$ properties. In the following, we proceed to give a brief summary of the physics used in the simulations presented in Braun et al. (2014) (hereafter: BSN14) in section 2 . We explain how we post-processed the resulting snapshots from these simulations with our Lyman- $\alpha$ code in section 3 . In section 4 , we present our results, followed by discussion and conclusions in section 5 .

\section{Simulations of isolated disk galaxies}

We post-process snapshots from a simulation of an isolated disk galaxy that was presented by BSN14 as the 'ref' run. We refer the reader to this paper for the details and briefly summarize the main ingredients of these simulations here. The simulations were performed using the adaptive mesh refinement (AMR) code Nyx (Almgren et al. , 2013) with an effective resolution of $\sim 30 \mathrm{pc}$. The simulated galaxy resides within a box of $0.5 \mathrm{Mpc}$ size and is initialized as a purely gaseous disk without stars using an adiabatically stable, isothermal setup. Employed physics include

- self-gravity from gas and stars and additionally the gravitational potential of a static NFW-shaped dark matter halo; 
C. Behrens and H. Braun: Inclination dependence of Lyman- $\alpha$ properties in a turbulent disk galaxy

- gas dynamics using the piecewise parabolic method for the resolved motions and a subgrid scale (SGS) model for unresolved turbulence following Schmidt \& Federrath (2011);

- cooling from gas, metals, and dust;

- multiphase ISM consisting of a diffuse warm, a clumpy cold, and a hot phase for supernova ejecta;

- star formation at a rate depending locally on the inferred molecular fraction and the thermal/turbulent state of the gas;

- stellar feedback in the form of a combination of thermal and turbulent feedback from supernovae (SN) and thermal feedback from Lyman continuum heating both depending on the age of the stars;

- metal enrichment due to SN;

We stress that gas dynamics, multiphase ISM, star formation and stellar feedback model all couple to the turbulent subgrid model. The simulation covers 2 Gyr of the evolution of the disk. Starting from an initially smooth configuration, the gaseous disk evolves into a flocculent disk with transient spiral features due to the dynamical self-regulation of star formation and stellar feedback. With time a rather smooth and extended stellar disk forms that features several short lived and very few longer-lived stellar clusters. Along with the enrichment with metals, the SN feedback also drives a galactic outflow carrying colder disk material with it that mostly falls back onto the disk.

We use three different snapshots from the simulation, representing the state of the galaxy $1,1.5$, and 2 Gyr after initialization of the disk. In this regime, the star formation has settled to a self-regulated state. Details about these snapshots are given in table 1.

\section{Lyman- $\alpha$ transport post-processing}

We use a standard Monte-Carlo approach to post-process the BSN14 simulations. We only summarize the additional physics and the implementation of the radiative transfer here, for a more thorough description we refer the reader to Dijkstra et al. (2006), Verhamme et al. (2006), Behrens \& Niemeyer (2013) among others.

Our code which, first presented in Behrens et al. (2014), is based on the BoxLib ${ }^{1}$ framework which is also the basis of Nyx (Almgren et al. , 2013). This makes it straightforward to postprocess the BSN14 simulation data, apart from a few physica prescriptions that we need for the purpose of performing the radiative transfer. We describe these in detail in the following paragraphs. For comparison with VDB12, we closely follow their setup if possible.

\subsection{Ionization state}

The BSN14 simulations do not explicitly trace the ionized fraction of neutral hydrogen. We therefore use the publicly-available code Cloudy ${ }^{2}$ to produce tables that yield the neutral fraction as a function of thermal energy, metallicity and total density in collisional ionization equilibrium (Ferland et al., 1998).

\subsection{Emissivity}

In order to run the Monte-Carlo simulation, we need to prescribe an initial spatial and spectral distribution of tracer photons on the AMR grid. The BSN14 model already contains a field for

\footnotetext{
${ }^{1}$ https://ccse.lbl.gov/BoxLib/

${ }^{2}$ http://www.nublado.org/ (Version 08.00)
}

the emissivity of Lyman continuum (LyC) photons from young stars. These trace the number density of Lyman- $\alpha$ photons, since ionization of neutral hydrogen by LyC followed by case-B recombination yields Lyman- $\alpha$ radiation. We assume here that this conversion happens locally, resulting in a direct proportionality between LyC photon density and Lyman- $\alpha$ photon density. As input spectrum, we use a Gaussian with a width of $10 \mathrm{~km} / \mathrm{s}$, centered around zero in the restframe of the given gas cell.

Additionally, we follow the radiative transfer of continuum photons near the Ly $\alpha$ line to calculate the EW distribution. Continuum photons are emitted with the same spatial distribution as Lyman- $\alpha$ photons, but with a flat spectrum extending out to $\pm 2 \times 10^{4} \mathrm{~km} / \mathrm{s}$ around the $\operatorname{Ly} \alpha$ line center. The total number of Ly $\alpha$ /continuum photons launched per snapshot is about $1.8 \times 10^{7}$.

\subsection{Lyman- $\alpha$ equivalent width}

To infer EWs from our radiative transfer simulations, we need to determine the ratio of emitted line and continuum flux which is equivalent to setting the intrinsic EW. This translates to a condition for the constant luminosity of continuum photons $L_{c}$ per wavelength bin $\Delta \lambda$ and the total intrinsic Lyman- $\alpha$ luminosity $L_{\alpha}$,

$\frac{\Delta L_{c}}{\Delta \lambda}=L_{\alpha} / E$

where $E$ is the desired intrinsic EW. In terms of the implementation, this condition fixes the ratio of continuum- and Lyman- $\alpha$ photons. We set the intrinsic EW to $200 \AA$. We note that we do not derive this value from the star formation rate and the stellar mass.

\subsection{Dust distribution}

Since the BSN14 simulations track the metallicity $Z=\rho_{m} / \rho$ where $\rho$ is the total density and $\rho_{m}$ is the metal density, but not the dust grain number density $n_{D}$, we have to implement a model for the distribution of dust. We slightly modify the model used by VDB12 (their eq. 3). The fraction of metals that settles in dust grains is assumed to be proportional to the neutral fraction of hydrogen $f_{N}$ and to the dust-to-metal ratio $R_{\text {dust/metal }}$ which is assumed to be 0.3 based on an empirical value for our own Galaxy (Inoue, 2003):

$n_{D}=f_{N} R_{\text {dust } / \text { metal }} Z \rho / m_{\text {grain }}$

Since the transition between the fully ionized and the fully neutral state is sharp, this model yields effectively similar results to the one used by VDB12. Employing their model would change the dust content only by a few percent. VDB12 use a value of $3 \times 10^{-17} \mathrm{~g}$ for the typical grain mass. Employing this value of the dust grain mass renders our galaxy a complete Ly $\alpha$ absorber (at least for the $1 \mathrm{Gyr}$ snapshot) with EWs consistently below zero. This is plausible since our galaxy is about a factor of 10 more massive than the one in VDB12. Since we are interested in the detailed inclination effect here and have no access to a less massive galaxy run, we scaled the total dust grain number density in our galaxy by a factor of about 25 , i.e. reducing the optical depth due to dust by this factor. This was implemented by simply scaling the dust grain mass to $m_{\text {grain }}=8 \times 10^{-16} \mathrm{~g}$ without changing the cross section. We call this model the 'fiducial' one, but we comment on the results for the runs with the VDB12 model that we will call the 'realistic' runs. 
C. Behrens and H. Braun: Inclination dependence of Lyman- $\alpha$ properties in a turbulent disk galaxy

Table 1. Physical parameters of the used snapshots

\begin{tabular}{cccccc}
\hline \hline Age & $M_{g}\left(M_{\odot}\right)$ & $M_{H I}\left(M_{\odot}\right)$ & $Z\left(Z_{\text {sol }}\right)$ & $M_{D}\left(M_{\odot}\right)$ & SFR $\left(M_{\odot} / y r\right)$ \\
$1 \mathrm{Gyr}$ & $6.8 \times 10^{9}$ & $4.1 \times 10^{9}$ & 0.47 & $1.1 \times 10^{7}$ & 2.8 \\
$1.5 \mathrm{Gyr}$ & $5.8 \times 10^{9}$ & $3.4 \times 10^{9}$ & 0.56 & $1.08 \times 10^{7}$ & 2.2 \\
$2 \mathrm{Gyr}$ & $5.1 \times 10^{9}$ & $2.9 \times 10^{9}$ & 0.58 & $0.96 \times 10^{7}$ & 1.7 \\
\hline
\end{tabular}

For the interaction cross section and reemission phase function, we follow Schaerer et al. (2011), using similar parameters, i.e. an albedo $Q=0.5$, and a Greenstein phase function parameter $G=0.7$

\subsection{Influence of the turbulent subgrid scale energy}

The Doppler parameter $b$ governs the width of the associated Voigt profile. The turbulent contribution to it is introduced as

$b=\sqrt{v_{t h}^{2}+v_{t u r b}^{2}}$

where $v_{t h}$ is the typical (RMS) thermal velocity in the gas, while $v_{\text {turb }}$ is the turbulent velocity component in the cell. While VDB12 assume a constant turbulent velocity here, we derive the typical (RMS) turbulent velocity of each cell directly from its turbulent energy $E_{t}$,

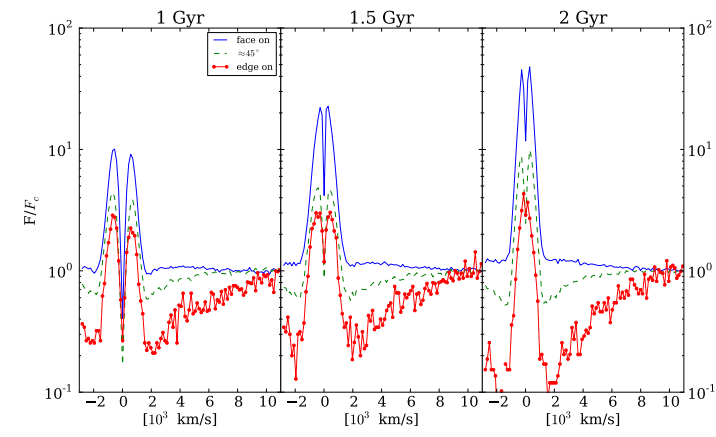

Fig. 2. Emerging spectra for all three snapshots (left to right: $1 / 1.5 / 2$ Gyr). We normalize the spectral flux to the continuum far away from the line and show it for three different inclinations: Face-on (solid line), edge-on (dotted line) and at about $45^{\circ}$ (dashed line).

$v_{t u r b}=\sqrt{2 E_{t}}$

\subsection{Morphology}

In Fig. 1, we show the projected HI density, the projected dust density, and the projected distribution of emission resulting from the prescriptions outlined above. The $1 \mathrm{Gyr}$ snapshot (upper plots) shows an unordered density structure, a dense network of individual bubbles and walls. In comparison, the $2 \mathrm{Gyr}$ snapshot (bottom plots) shows a concentrated profile with larger underdense and overdense regions and hints of spiral structures. While the 1 Gyr snapshot shows extended emission in smaller clumps (top right), the emission in the $2 \mathrm{Gyr}$ snapshot (bottom right) is more concentrated to a few clumps near the center. The 1.5 Gyr snapshot lies in between. As expected, the dust density approximately follows the neutral hydrogen density in all cases. As implied by the data in table 1 , the dust content does not vary significantly between the snapshots. This implies that at least at this stage, the accumulation of metals does not lead to an increase in dust mass due to hots winds carrying metals away from the cool disk where dust grains could form.

\section{Results}

\subsection{The fiducial simulations}

The spectra that emerge using the fiducial prescriptions outlined above for the three snapshots (left/center/right panel for $1 / 1.5 / 2$ Gyr) are shown in Fig. 2. Each panel features the spectrum obtained in face-on/edge-on direction (solid line/dots) and for $\approx 45^{\circ}$ with respect to the disk (dashed line). All spectra resemble the typical double-peak spectrum with slightly asymmetric peaks. The double peak, however, is barely visible in the $2 \mathrm{Gyr}$ data. Comparing the three snapshots, we find that the normalized line flux is largest in the $2 \mathrm{Gyr}$ run viewed face-on. The trough at line center is very prominent in the $1 \mathrm{Gyr}$ snapshot and gradually decreases in depth and width in the two other snapshots. The position of the strongest peak changes; while it is the red peak for 1

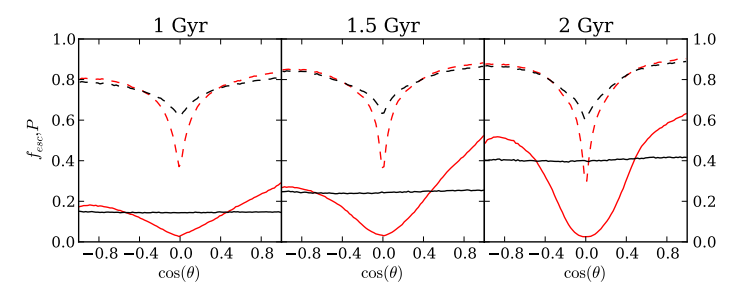

Fig. 3. Escape fraction as a function of inclination at escape in gray/red for continuum (dashed) and Ly $\alpha$ (solid). In black we show the probability of transmission given that the photon was initially emitted with inclination $\cos \theta$ again for continuum (dashed) and Ly $\alpha$ (solid). Data is shown for the $1 \mathrm{Gyr} / 1.5 \mathrm{Gyr} / 2 \mathrm{Gyr}$ snapshot from left to right.

Gyr and 1.5 Gyr and the edge-on spectrum for 2 Gyr, the face-on spectrum for $1.5 \mathrm{Gyr}$, the face-on and the $\approx 45^{\circ}$ spectrum at 2 Gyr show a slightly higher blue peak. As VDB12 point out, the red peak might be enhanced by scattering in outflowing material (also see Verhamme et al., 2006). Inflowing material would produce a blue peak. We believe the peaks of the simulated spectra to be related to the fact that both inflowing and outflowing gas exist at the same time. Outflowing gas is driven outwards by feedback, and may fall back onto the disk later. They vary in strength and direction according to the recent star formation history and the entailing stellar feedback in the disk.

Fig. 3 shows the distribution of escape directions (gray/red) and the probability of a photon escaping with a certain initial inclination (black) as a function of $\cos \theta$, where -1 and 1 correspond to face-on from below (along the $-z$-axis) and above the disk (along the $+z$-axis). Edge-on corresponds to a value of $\cos \theta=0$. We show the data for both continuum photons (dashed) and Ly $\alpha$ photons (solid) for each of the three snapshots (1/1.5/2 Gyr from left to right). For Ly $\alpha$ photons, the probability to escape is independent of the initial direction of the photon, 
C. Behrens and H. Braun: Inclination dependence of Lyman- $\alpha$ properties in a turbulent disk galaxy
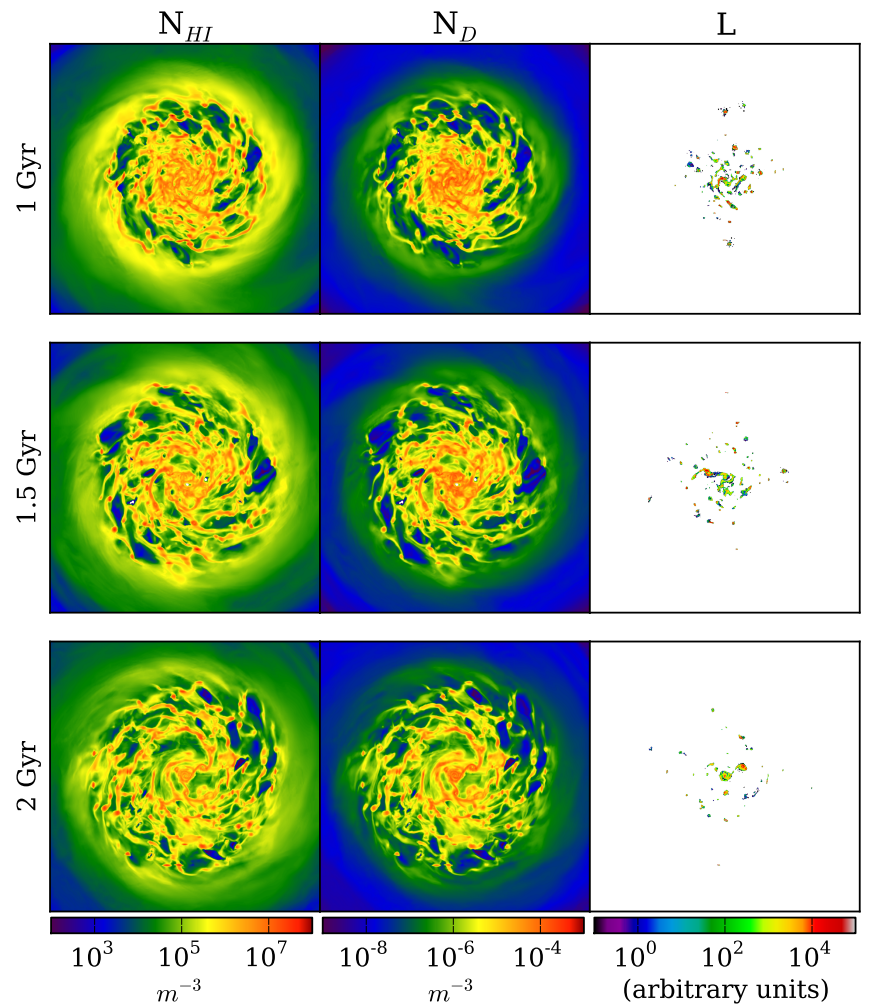

Fig. 1. Projected neutral hydrogen density/dust density/emissivity in the disk (left/center/right) for the three snapshots at $1 / 1.5 / 2$ Gyr (top/center/bottom). Each plot shows the central $40 \mathrm{kpc}$ of the disk.

as also found by VDB12 (their Fig. 8). Similar to their results, Ly $\alpha$ photons show in general a strong tendency to escape faceon, but we observe that the slope is different for negative/positive inclinations. For the continuum photon, both a weak dependence of the escape fraction and the escape direction on inclination is found, similar to VDB12. The amplitude of the inclination effect is different, and the exact shape and steepness of the dependency differs. For example, for the $1 \mathrm{Gyr}$ snapshot, the escape fraction for $\operatorname{Ly} \alpha$ photons is a rather shallow and nearly linear function, but the total range of escape fractions spans about a factor of 10 , which is comparable to VDB12. At $2 \mathrm{Gyr}$, the variation is about a factor of 60 , and the distribution is also much steeper.

The Ly $\alpha$ mean escape fraction varies by a factor of $\approx 3$ across the snapshots, while the continuum escape fraction approximately remains constant: The mean escape fractions of Ly $\alpha$ photons are $14 / 24 / 41 \%$ at $1 / 1.5 / 2$ Gyr for the Ly $\alpha$ photons, while $75 / 79 / 81 \%$ of the continuum photons escape, respectively. The Ly $\alpha$ escape fractions are broadly consistent with former studies at lower resolution (e.g Laursen et al., 2009), but in some tension with VDB12: they infer $22 \%$ for continuum and $5 \%$ for Ly $\alpha$ photons (for their simulation G2). This difference is readily explained by our having scaled the dust content down to study the inclination effect. In fact, in the realistic case, we obtain lower escape fractions of $0.2-15 \%$ consistent with our galaxy being more massive.

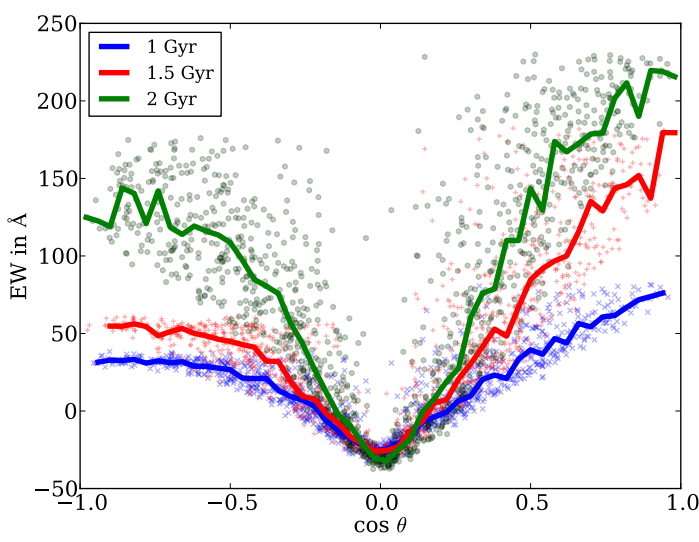

Fig. 4. Ly $\alpha$ EW as it would be observed along lines of sight randomly chosen as a function of $\cos \theta$. The lines show the binned median.

Fig. 4 shows the EW distribution as a function of $\cos \theta$ where each data point corresponds to a randomly chosen observation direction. The EW varies drastically from one line of sight to another and shows a general trend to be larger at larger absolute inclination as expected. Additionally, it varies systematically from 
C. Behrens and H. Braun: Inclination dependence of Lyman- $\alpha$ properties in a turbulent disk galaxy

snapshot to snapshot: while the maximum EW is around $70 \AA$ for the 1 Gyr snapshot, it peaks around $230 \AA$ for the 2 Gyr run. The asymmetry between observers above and below the disk is also present here. While for the $1 / 1.5 \mathrm{Gyr}$ run the EW is significantly suppressed for observers with $\cos \theta<0$, this suppression seems to be reduced in the $2 \mathrm{Gyr}$ run. For each observer, we collected all photons within $\pm 10^{\circ}$ of the line of sight to have sufficient statistics. We caution the reader that the exact minimum/maximum values and the strength of fluctuations changes depending on this angular range; using $\pm 20^{\circ}$ instead of $\pm 10^{\circ}$ for example would shift the minimum (maximum) value to about 0 (200) $\AA$ for the 2 Gyr snapshots. Comparing with the results of VDB12 (their Fig. 10), similar trends are seen, but both the range of values and the shape of the distribution are different. The scatter in our plots is generally larger, especially for the 1.5 and 2 Gyr snapshot. While it is natural to have different EW distributions in our work and VDB12 since both mean escape fraction and escape fraction as a function of inclination are different, some of the differences might also come from a different choice of the angular range for each observer. A large angular range effectively averages out local fluctuations like cavities with small solid angles.

In Fig. 5, we show the spatial distribution of both transmitted Ly $\alpha$ photons (top) and the photons that were destroyed by dust (bottom plots). The plots show the binned distributions of the spots of last interaction with the gas, i.e. the spots of last scattering (before escape) for the transmitted photons and the locations of absorption for the destroyed photons. For all snapshots, transmission is diffuse except for few clumps that exhibit larger transmission. Comparing the distribution of the transmitted photons with the intrinsic emissivity in Fig. 1 (right), we see that most of the emitting clumps do not show transmission. Most of the photons launched in these places are destroyed by dust, which is clearly visible in the distribution of the absorbed photons. The distribution of the destroyed photons in Fig. 5 resembles the emissivity map in Fig. 1, indicating that many of the emitted photons are destroyed locally. This is expected, since the emitting clumps are typically very dense.

Comparing the spatial distribution of the transmitted photons among the three snapshots, the transmission is more spatially concentrated in the 1.5 and 2 Gyr snapshots which is partly explained by the emissivity being more concentrated in these snapshots. Especially for the 2 Gyr snapshot, we see that most of the Ly $\alpha$ photons escape from three central clumps, e.g., the largest of those clumps contributes about half of all transmitted Lya photons.

To conclude, we state that there is a distinct top-bottom asymmetry in all snapshots, and that the snapshots differ drastically in terms of Ly $\alpha$ properties. In the following, we investigate these results further with focus on the differences between the 1 Gyr and 2 Gyr snapshot.

\subsubsection{Differences between the snapshots}

From table 1, it is clear that the total neutral gas mass decreases with time due to ongoing star formation. Since a smaller neutral gas mass implies a lower optical depth, this partly explains the differences between the subsequent snapshots. One can check this by artificially decreasing the neutral gas mass in the $1 \mathrm{Gyr}$ snapshot. Reducing the gas mass by a factor of 2 in the $1 \mathrm{Gyr}$ snapshot enhances the EW (and escape fraction) by about $40 \%$ (see section 4.3 below), but this is not enough to explain the boost observed between the 1 and 2 Gyr snapshot. This result

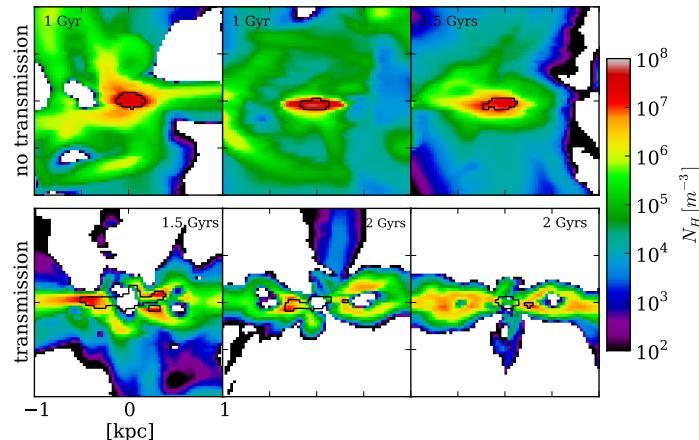

Fig. 6. Examples for density slices through the disk at the location of various emission spots. The disk lies along the $x$-axis in the plots. Top row: examples for spots that do not exhibit significant transmission. Bottom row: spots that show transmission. The black contours indicate the approximate center of emission in each slice.

already gives a hint that the driving factor here is not only the gas mass, but also the detailed morphology of the disk. To understand this local morphology, we analyzed the data of emitted and transmitted photons by detecting large emission spots and correlating them with clumps of escaping Ly $\alpha$ photons. Clumps are detected by binning the data onto a grid, applying a luminosity threshold and adding up all bins that are spatially connected. This yields a set of emission clumps that have counterparts in transmission and another set of emission spots that lack a counterpart.

The high-transmission spots are located in the vicinity of low-density regions due to a highly disturbed environment, or are close to low-density cavities connecting the inner, dense part of the disk with the ionized region around the disk. A few examples of slices of the density distribution around the centers of emission within the disk are shown in Fig. 6. The origin of the cavities is the star formation feedback. As turbulent and thermal energy is released into the disk by $\mathrm{SN}$, high-temperature, lowdensity bubbles form and expand until they drain hot gas and pressure through leakages into their surroundings above and below the galactic disk. For example, the environment of the largest emission clump in the 2 Gyr snapshot (see Fig. 6, bottom center) shows a bubble in the center, connected to the outer parts of the disk at the bottom. The column density for direct escape to the top is also low for some lines of sight. Emission spots that are located in a cavity usually have counterpart in the transmission, while those that lack a counterpart in the transmitted Ly $\alpha$ are typically located in, or surrounded by, dense regions in which the Ly $\alpha$ photons scatter until they are eventually absorbed.

The discrimination of emission spots between those with and without counterparts in transmission is also a distinction between different evolutionary stages of star-forming regions and the environments. In the early stages, the LyC-emitting stars reside in a dense environment. Later, as the first massive stars die in SN explosions a bubble or cavity begins to form which at the same time stalls star formation locally. As the LyC-emitters are short-lived, large cavities are frequently found hosting only negligible Ly $\alpha$ emission.

The number of large cavities associated with strong emission spots is lower in the $1 \mathrm{Gyr}$ snapshot compared to that of the 1.5 or 2 Gyr snapshots. This leads, in concert with the already mentioned higher neutral gas content, to the observed lower escape 
C. Behrens and H. Braun: Inclination dependence of Lyman- $\alpha$ properties in a turbulent disk galaxy

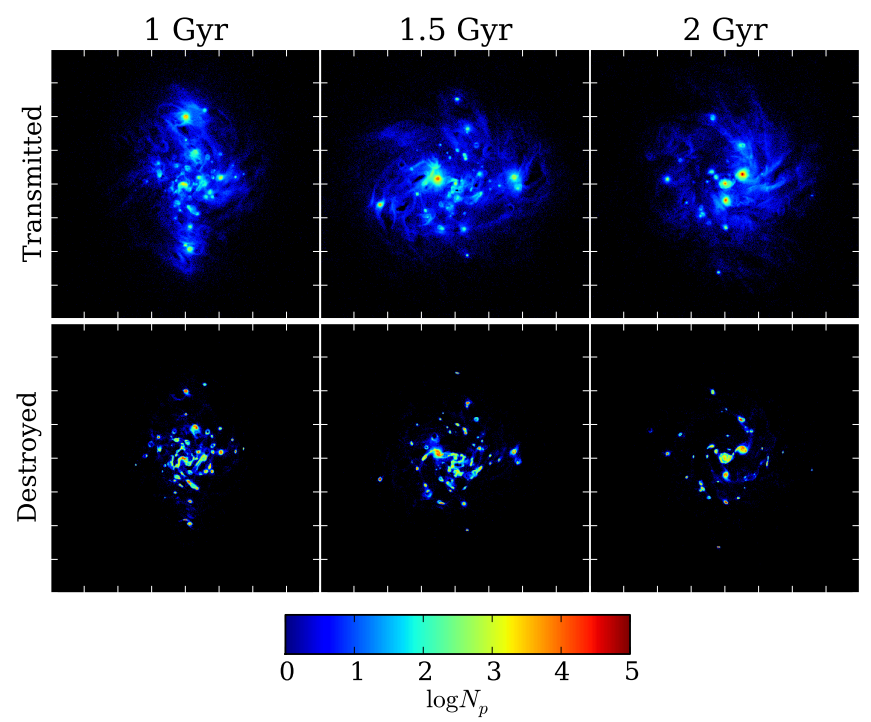

Fig. 5. Spatial distribution of escaping photons (top) and absorbed photons (bottom) for the snapshot at $1 / 1.5 / 2$ Gyr (left/center/right). For the transmitted photons, the distribution is given by the points in the simulation box where photons scattered just before it left the box. For the destroyed Ly $\alpha$ photons, we binned the positions at which photons were absorbed. Each plot shows the central $40 \mathrm{kpc}$ of the disk.

fraction and the lower EW in the 1 Gyr run. The strongest emission spots correlated with cavities found in the late snapshots arise from massive stellar clusters that locally dominate gravity and, as a consequence, host intermittent violent star formation. Those stellar clusters are assembled via mergers of marginally bound clusters, which are the remnants of star forming regions in the low-metallicity regime of star formation, as described by BSN14. On the one hand, the Ly $\alpha$ emitting phase in small clusters ends roughly around the time at which the development of a cavity starts, such that the Ly $\alpha$ radiation is most probable to be obscured by the clump hosting the star formation originally. Ly $\alpha$ radiation from small clusters contributes to the transmitted flux only for the short time between the formation of a bubble and the termination of $\operatorname{Ly} \alpha$ emission after star formation stops (and the formed massive stars die), since the small clusters are created during a single event of star formation without any subsequent star formation. On the other hand, feedback launched at the location of prominent emission spots by stars that were formed during previous duty cycles of a large cluster already prepared a cavity reaching far into the disk's surroundings. This preexisting cavity serves as an escape path for radiation emitted by later generations of stars, such that this kind of emission spot is prominent in the transmission. This explanation implies that for both small and massive clusters, the contribution to Ly $\alpha$ transmission varies on the timescale of the star formation activity ( $\sim$ few $10 \mathrm{Myr}$ ). For massive clusters, this is identical to the timescale of the duty cycle; for small clusters, it is the lifetime of the star-forming region.

\subsubsection{The top-bottom asymmetry}

The top-bottom asymmetry can be readily explained on the basis of the last section. Most of the radiation leaking from the emission spots (see Fig. 6 for examples) escapes toward the top of the simulation box. The excess of the EW at negative $\cos (\theta)$ in

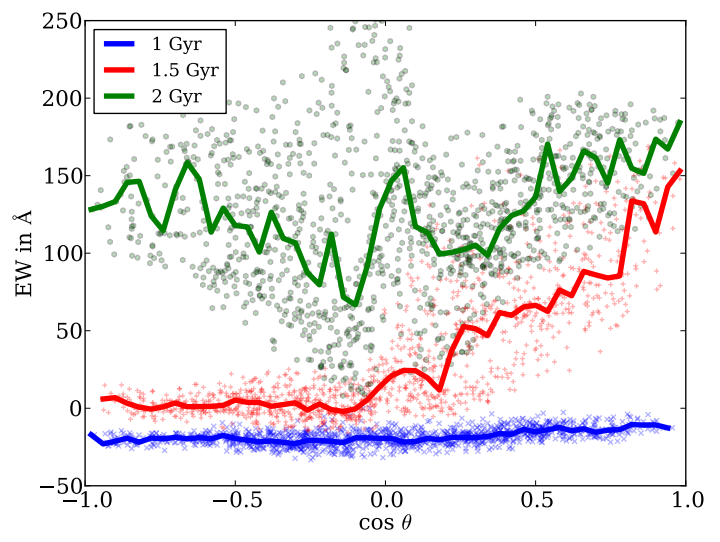

Fig. 7. Same as fig. 4, but for the dust prescription used in VDB12, i.e. without the scaling of the dust content we invoked for the fiducial runs.

the 2 Gyr run compared to the younger snapshots (see Fig. 4) is caused mostly by the most active emission spot in the 2 Gyr run (see bottom center panel of Fig. 6) that transmits equally to both sides ( $\pm z$ direction). This is not the case e.g., for the most prominent emission spot in the $1.5 \mathrm{Gyr}$ run (bottom left panel in Fig. 6 ), which contributes about $40 \%$ to the total $\operatorname{Ly} \alpha$ transmission. To further illustrate this asymmetric behavior, we plot again the distribution of transmitted Ly $\alpha$ photons, this time for photons escaping within $\pm 10^{\circ}$ to the top/bottom in Fig. 8 (top/bottom panels) for the snapshots (1/1.5/2 Gyr at the left/right). For comparison, plots for the photons escaping edge-on (middle row of Fig. 8) are also shown. Many of the strongly transmitting spots preferentially transmit to the top. 
C. Behrens and H. Braun: Inclination dependence of Lyman- $\alpha$ properties in a turbulent disk galaxy

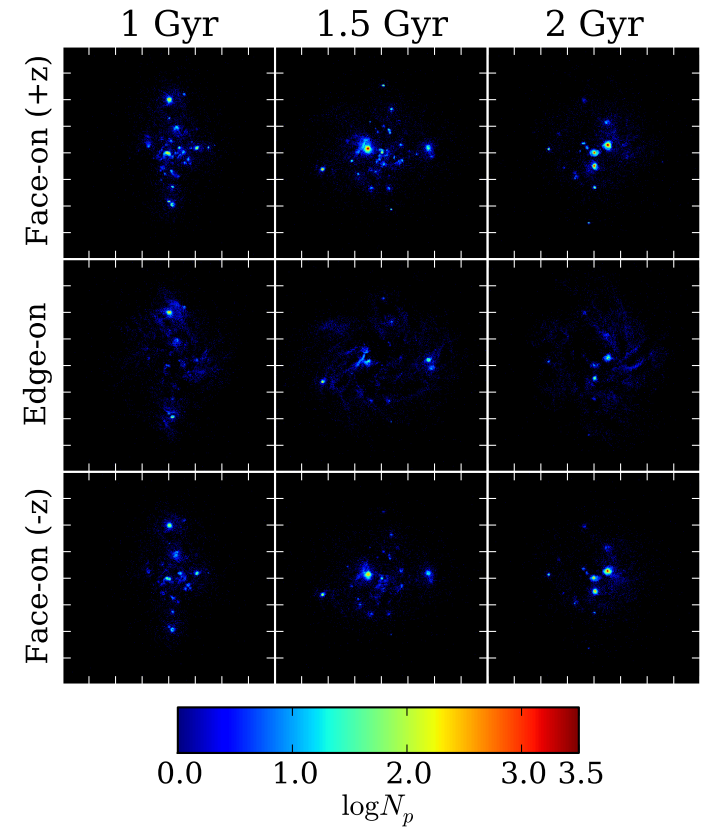

Fig. 8. In this plot we show the spatial distribution of transmitted Lyo photons for the snapshots at $1 / 1.5 / 2 \mathrm{Gyr}$ (left to right). In contrast to Fig. 5 , we show only the photons that escaped face-on toward the positive $z$-axis (top), edge-on (middle), and face-on toward the negative $z$-axis (bottom). Photons within a range of $\pm 10^{\circ}$ of the principal direction were considered. Shown are the central $40 \mathrm{kpc}$ of the disk.

It is plausible that the ongoing feedback processes, namely SN bubbles blowing up, slightly prefer a particular direction even on longer timescales. The feedback-driven bubbles follow the path of least resistance. This path through the surroundings of the disk is likely to be a fossil remnant of the path of another, more or less recent outflow (see Section 4.1.1). So if the outflows launched from a particular massive stellar cluster had a top-bottom asymmetry initially due to massive obstacles in one direction, this asymmetry may persist for a few dynamical timescales of the disk, i.e. a few $100 \mathrm{Myr}$.

\subsection{The realistic simulations}

Our results also apply if we do not scale the dust content as we have done for the fiducial runs. In contrast, cavities become even more effective in boosting escape of $\operatorname{Ly} \alpha$ photons in this case. We show the EW distribution for the 'realistic' case in 7. As has been mentioned before, the 1 Gyr run shows Ly $\alpha$ absorption in this case, with a $\operatorname{Ly} \alpha$ escape fraction of $0.2 \%$ on average. The $1.5 / 2$ Gyr runs, on the other hand, show a very distinct dependence of EW on orientation. Only the cavities identified in the fiducial runs do significantly transmit $\operatorname{Ly} \alpha$ and increase the average Ly $\alpha$ escape fraction to $4(15) \%$ in the 1.5 (2) Gyr run. The $1.5 \mathrm{Gyr}$ snapshot therefore shows an enhanced asymmetry related to the orientation of the main transmitting cavity. Similar to the fiducial run, the 2 Gyr run shows transmission both to the top and to the bottom. The strong fluctuations in EWs measured edge-on are an artifact coming from our not applying a flux limit: The escape

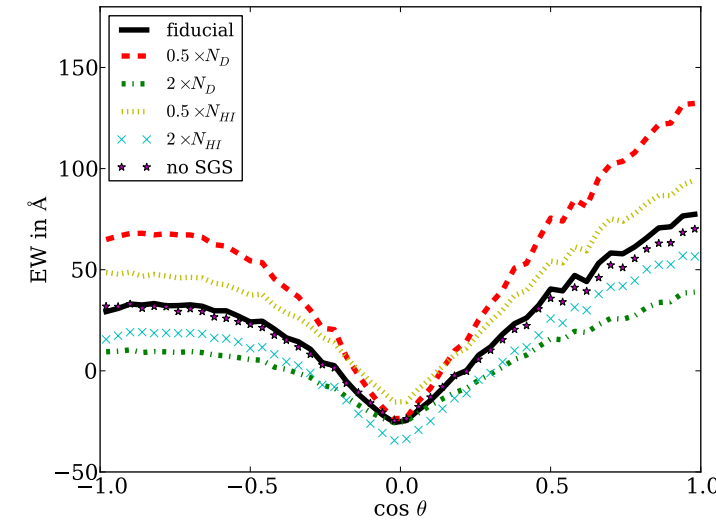

Fig. 9. Same as Fig. 4, but for different parameter variations of the 1 Gyr snapshot. We plot the median lines here only.

fraction for both $\operatorname{Ly} \alpha$ and continuum photons is below $0.5 \%$ in this region. We conclude that the significance of cavities for the escape of Ly $\alpha$ photons is a robust feature.

\subsection{Varying parameters}

We performed an additional set of 5 radiative transfer simulations on the $1 \mathrm{Gyr}$ data varying a single fundamental parameter at a time to explore its effect on the inferred Ly $\alpha$ properties of the galaxy. In particular, the dust or neutral gas content were scaled up or down by a factor of 2 . In an independent run, the SGS model was switched off to ignore the effects of small-scale turbulence on the radiative transfer. We show only the results for the EW distribution in Fig. 9. For comparison, the black line shows the fiducial case. The SGS model affects the EW distribution least. Since disabling it effectively makes the Voigt profile narrower and higher, the simulation without SGS model (stars) shows slightly lower EWs. Photons in the line center are scattered more effectively, and excursions to frequencies far away from the line center become less probable since the velocities of the scattering atoms are statistically lower.

Interestingly, reducing/enhancing the dust content by a factor of 2 (dashed/dashed-dotted line) has the largest effect on the EW distribution, reducing/enhancing the maximum by a factor of a few. Reducing/enhancing the neutral hydrogen content by a factor of 2 (crosses/dashed line) affects the EW distribution only by a factor of 2 . The relative sensitivity to dust can be made plausible by considering that, to first order, the hydrogen density increases the mean pathlength. Larger pathlengths make absorption exponentially more probable, and the same holds for higher dust densities. On the other hand, the mean pathlength is not necessarily a linear function of the hydrogen density. Adams (1975) has shown that at least for simple geometries, the pathlength scales sub-linearly with the hydrogen density.

\subsection{Dust-free case}

As a last variation, a simulation ignoring dust in the radiative transfer was performed. This is physically inconsistent with the evolution and history of our simulated galaxy. However, it provides some information about the inclination dependence in extremely metal-poor regimes, for example, in young starburst 
C. Behrens and H. Braun: Inclination dependence of Lyman- $\alpha$ properties in a turbulent disk galaxy

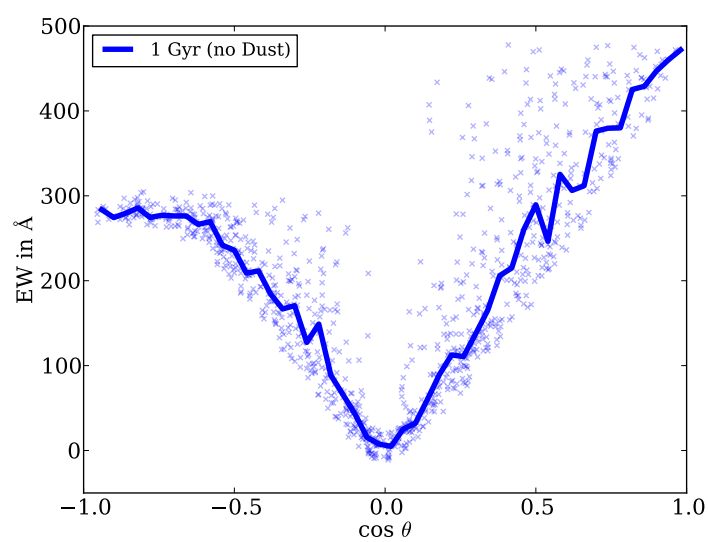

Fig. 10. Same as Fig. 4, but for the 1 Gyr snapshot without dust.

galaxies. We show the EW distribution for the $1 \mathrm{Gyr}$ snapshot ignoring dust effects in Fig. 10. The absence of dust implies that all photons launched escape the galaxy eventually. While the average EW recovers the chosen intrinsic EW of $200 \AA$, there are large variations between individual lines of sight. A pronounced inclination effect is present here as well. The intrinsic EW is exceeded by a factor of 2 and more for face-on observers, while edge-on observers see EWs as low as $-10 \AA$ A. The excess above the intrinsic EW in face-on directions is expected. This is a geometrical effect arising from the higher probability for photons to escape in face-on directions compared to edge- on directions.

\section{Discussion and conclusions}

We investigated Ly $\alpha$ radiative transfer in simulations of an isolated disk galaxy with detailed star formation, feedback and a multiphase ISM, using snapshots of the 'ref' simulation from BSN14 at different timesteps.

Our results are in broad agreement with VDB12. We find a strong inclination dependence of Ly $\alpha$ properties in terms of flux, EW distribution, and spectra. This is readily explained by the different optical depth in directions parallel and perpendicular to the disk.

As a new result, we find variations of these properties between the snapshots at $1,1.5$, and 2 Gyr. For example, the minimum (maximum) of the observed EW varies from -20 to 0 (70 to 240$) \AA$ in our fiducial simulations depending on the snapshot. While this can be partly attributed to the lower neutral hydrogen density of the disk at later times, detailed analysis reveals the importance of supernovae-blown cavities within the disk which reduce the optical depth for photons locally by orders of magnitude (see Fig. 6). The transmitting cavities have a lifetime of several $10 \mathrm{Myr}$ corresponding to the period of star formation and stellar feedback. We conjure that the origin of the variations is partly working on timescales of the star-formation activity. The occurrence of cavities is directly connected to the recent local star formation history, and the majority of those that become important as preferred pathways are connected to massive stellar clusters that iteratively host star formation. This suggests that it is difficult to quantify the statistical inclination dependence of Ly $\alpha$ photons for such simulations in a meaningful way, because it would be necessary not only to derive it for a large set of realizations of galaxies, but also to keep track of the evolution of particular emission spots. Compared to our simplified models presented in Behrens et al. (2014) which also feature cavities, the isolated disk galaxy has Ly $\alpha$ properties arising from the superposition of multiple, transmitting cavities and a diffuse component, with the cavity component becoming more important at higher dust content.

On the observational side, our results suggest that the scatter of observed Ly $\alpha$ properties can be severely enhanced by this variability on relatively short timescales. To overcome this, one would have to identify individual cavities leaking $\operatorname{Ly} \alpha$ emission directly, which is difficult at high redshifts where LAEs are not spatially resolved. As described in (Behrens et al., 2014), it might be possible to identify LAEs for which the Ly $\alpha$ flux is dominated by cavities by their excess flux at line center (see also Fig. 2). Statistically, the distribution of EWs in a homogeneous sample of star-forming galaxies identified by an unbiased star formation indicator should show a scatter attributed to the suggested temporal variation.

It will be a further challenge to add two important aspects of realistic galaxies and Ly $\alpha$ transport: the immediate surroundings of the galaxy as a source for infalling material, and the IGM scattering Ly $\alpha$ photons out of a specific line of sight and frequency. For example, if streams of cool, metal-poor gas were present, we would assume to have a bluer spectrum for photons emerging from the locations where the streams penetrate the disk. Additionally, in these dense streams, the turbulent motion of the gas might play a significant role. Including these features might reduce the variability of the disk, since there might be less lines of sight that have low neutral hydrogen column density. Additionally, following the evolution of the Ly $\alpha$ properties over of a galaxy in much smaller timesteps will be necessary to further investigate this temporal variation.

We stress that the inclination dependency of Ly $\alpha$ properties is not only found in high-resolution simulations with detailed ISM physics, but also in cosmological simulations with kpc resolution. For example, Behrens \& Niemeyer (2013) find a $\sim 15 \%$ increase in flux face-on compared to flux escaping edgeon. This underlines how important it is to understand correlations between inclination and large-scale structure, i.e. galaxy alignment (Hirata, 2009) and the large-scale density and velocity field (Zheng et al., 2010, 2011).

Acknowledgments $\mathrm{C}$. Behrens and $\mathrm{H}$. Braun were financially supported by the CRC 963 of the German Research Council. The authors thank J. Niemeyer for helpful comments and discussions.

\section{References}

Adams, T. F. 1975, ApJ, 201, 350-351

Adams, J. J., Blanc, G. A., Hill, G. J., et al. 2011, ApJS, 192, 5 Ahn, S.-H., Lee, H.-W., \& Lee, H. M. 2003, MNRAS, 340, 863

Almgren, A. S., Bell, J. B., Lijewski, M. J., Lukić, Z., \& Van Andel, E. 2013, ApJ, 765, 39

Behrens, C., \& Niemeyer, J. 2013,A\&A, 556, A5.

Behrens, C., \& Niemeyer, J. 2013,A\&A, 556, A5.
Behrens, C., Dijkstra, M., \& Niemeyer, J. 2014,A\&A, 564, A77.

Behrens, C., Dijkstra, M., \& Niemeyer, J. 2014,A\&A, 564, A77.
Braun, H., Schmidt, W., Niemeyer, J.C., \& Almgren, A.S. 2014, MNRAS, submitted

Dijkstra, M., Haiman, Z., \& Spaans, M. 2006, ApJ, 649(1), 1436.

Dijkstra, M. \& Wyithe, J. S. B. 2010, MNRAS, 408, 352-36

Dijkstra, M. 2014, submitted to PASA, arXiv: 1406.7292

Duval, F., Schaerer, D., Östlin, G. \& Laursen, P. 2014, A\&A, 562, A52

Faucher-Giguère, C.-A., Kereš, D., Dijkstra, M., Hernquist, L., \& Zaldarriaga, M. 2010, ApJ, 725, 1

Ferland, G. J., Korista, K. T., Verner, D. A., Ferguson, J. W., Kingdon, J. B. \& Verner, E. M. 1998, PASP, 110, 761-781.

Gronke, M. \& Dijkstra, M. 2014, submitted to MNRAS, arXiv:1406.6709 
C. Behrens and H. Braun: Inclination dependence of Lyman- $\alpha$ properties in a turbulent disk galaxy

Hill, G. J., Gebhardt, K., Komatsu, E., Drory, N., MacQueen, P. J., Adams, J.,

Blanc, G. A., Koehler, R., Rafal, M., Roth, M. M., Kelz, A., Gronwall, C.

Blanc, G. A., Koehler, R., Rafal, M., Roth, M. M., Kelz, A., Gronwall, C.,
Ciardullo, R. \& Schneider, D. P. 2008, Astronomical Society of the Pacific Conference Series, 399,115

Hirata, C. M. 2009, MNRAS, 399, 1074-1099

Inoue, A. K. 2003, PASP, 55, 901-909.

Laursen, P., Duval, F., \& Östlin, G. 2013, ApJ, 766, 124

Laursen, P., Razoumov, A. \& Sommer-Larsen, J. ApJ, 2009, 696, 853-869

Barnes, M. A., Haehnelt, M. G., Tescari, E. \& Viel, M. 2011, MNRAS, 416,

1723

Schmidt, W. \& Federrath, C. 2011, A\&A, 528, A106

Schaerer, D., Hayes, M., Verhamme, A. \& Teyssier R. 2011, A\&A, 531, A12

Tasitsiomi, A. 2006, ApJ, 645, 795-813

Verhamme, A., Schaerer, D., \& Maselli, A. 2006, A\&A, 460, 397

Verhamme, A., Dubois, Y., Blaizot, J., et al. 2012, A\&A, 546, A111

Verhamme, A., Orlitova, I.,Schaerer, D. \& Hayes, M. 2014, submitted to A\&A,

arXiv: 1404.2958

Yajima, H., Li, Y., Zhu, Q., \& Abel, T. 2012, MNRAS, 424, 884-901

Zheng, Z., Cen, R., Trac, H., \& Miralda-Escudé, J. 2010, ApJ, 716, 574

Zheng, Z., Cen, R., Trac, H., \& Miralda-Escudé, J. 2011, ApJ, 726, 38

Zheng, Z., \& Wallace, J. 2013, submitted to ApJ, arXiv:1308.1405 


\section{CHAPTER 5}

\section{Summary and Discussion}

In this thesis, we have focused on the work compiled in the three publications reproduced in chapter 4 . While the first publication (Behrens \& Niemeyer 2013) was concerned with possible contamination of LAE surveys by radiative transfer effects, the second (Behrens et al. 2014) focused on the inclination-dependent transmission of simplified models representing individual LAEs. The third publication (Behrens $\&$ Braun 2014) dealt with the detailed radiative transport of Lyman- $\alpha$ photons in a single, well-resolved, isolated disk galaxy and continued to investigate the dependency of Lyman- $\alpha$ properties on the line of sight of an potential observer. While these three publications probe a variety of spatial scales, ranging from several ten Mpc in the case of the cosmological simulation in the first work down to few ten Parsec in the last one, they share three important links.

First, they are connected to the problem of contamination of large-scale surveys employing LAEs as depicted in chapter 2. While Behrens \& Niemeyer 2013 explicitly considered the contamination by the large-scale density fields and kinematics, the effect of a possible tidal alignment of the galaxies' spin in the large-scale environment has been a motivation for studying the inclination dependence of Lyman- $\alpha$ radiation from an anisotropic LAE in Behrens et al. 2014, and the work in Behrens \& Braun 2014 extends this study to the case of a more detailed, realistic LAE.

This brings us to a second connection shared by all the work presented in this thesis: the importance of inclination-dependent escape of Lyman- $\alpha$ radiation. In particular, the work done on simplified models was motivated by the finding that in the cosmological simulation used in Behrens \& Niemeyer 2013, we found an 
inclination effect ${ }^{1}$ that was comparable in amplitude to the correlations with the large-scale structure in its amplitude, even though the ISM was resolved at scales of $\mathrm{kpc}$, and the emitters were assumed to be point-like. This underlines the importance of considering such effects.

Simulating these effects brings us to the third point, which is that all the work presented here touches the subject of LAE modeling and its significance to our understanding of what LAEs are and how their properties can be explained in a physically consistent way. While Behrens \& Niemeyer 2013 modeled LAEs on the background of a cosmological simulation, our simplified models (Behrens et al. 2014) focused more on understanding which effects arise from simple, anisotropic models. This allows to disentangle interaction in the IGM from the ISM scales. In the third publication, we modeled only one very detailed emitter and analyzed how the properties of such a realistic emitter change over time, in parts verifying our results from the simplified models. In particular, the importance of lines of sight with low optical depth for the inclination dependent escape in the shell with holes or cavity models of Behrens et al. 2014 has been found in supernovae-blown cavities in our realistic disk galaxy.

In the following, we will briefly summarize our main findings, possible caveats of the work presented and the future prospects.

We showcased work on the numerical Lyman- $\alpha$ photon transport in various environments. First, we performed simulations in a cosmological volume obtained from the MareNostrum-HORIZON run (Ocvirk et al. 2008) to study the contamination of cluster statistics derived from LAEs due to correlations of the observed Lyman- $\alpha$ properties and the large-scale environment of the emitters. This analysis deepens and extends the work by Zheng et al. 2010. We verify the existence of correlations between the observed fraction of Lyman- $\alpha$ flux and the large-scale density, velocity, density gradient, and the velocity gradient that have intuitive explanations. For example, we find that large-scale overdensities tend to reduce the observed fraction of Lyman- $\alpha$ radiation, due to the fact that photons in those regions are subject to more scatterings in the IGM, and the probability of those photons to be scattered by a nearby halo is higher. However, we do not find a significant deformation of the 2PCF,

1 We found a correlation between inclination of the dark matter spin direction and the observed Lyman- $\alpha$ fraction. Objects with spins parallel to the line of sight statistically have about $\sim 15 \%$ higher observed fractions compared to spins perpendicular to the line of sight. 
i.e., we do not find a significant contamination of the clustering signal. Although we cannot exclude the possibility that our non-detection originates from the limited volume $(50 \mathrm{Mpc} / \mathrm{h})$ of our simulations and would be detectable in a larger volume, we can show that at the lower redshift range we investigated $(z=2-4)$ compared to Zheng et al. $2010(z=5.7)$ and the higher numerical resolution that we have $(1 \mathrm{kpc}$ vs. $32 \mathrm{kpc}$ ), the correlations of Lyman- $\alpha$ properties with the large-scale quantities are weaker. In particular, the correlation of the line-of-sight velocity gradient with the observed fraction of Lyman- $\alpha$ emission is nearly a magnitude smaller in amplitude in our simulations. Additionally, all measured correlations become weaker at lower redshift in our simulations, which renders it improbable to find contamination at the redshifts probed by HETDEX and other large-scale surveys. To fully verify these findings, a high-resolution $(\leq 1 \mathrm{kpc})$ simulation with a larger volume $(\geq 100$ $\mathrm{Mpc} / \mathrm{h}$ ) would be necessary, including full treatment of the baryons and galactic processes like star formation and feedback. To date, no such simulation is available to us, although ongoing work by Jan-Frederik Engels in Göttingen might deliver a sufficient simulation. Additionally, using a large n-body simulation run by Stefan Gottloeber and collaborators ${ }^{1}$ in combination with a semi-analytic model of galaxy formation (Benson 2012) might be able to at quantify the impact on the clustering on a broader basis, although this approach will rely on modeling the radiation transport semi-analytically.

Apart from the sample size given by the simulation volume, our simulations in Behrens \& Niemeyer 2013 do not include dust and contain no other source of Lyman- $\alpha$ photons than star formation. It is a matter of debate to which extent a contribution from gravitational cooling to the Lyman- $\alpha$ flux is important, since it is unclear how much Lyman- $\alpha$ flux is generated in cold streams, and what the impact on the observational properties is: For example, Laursen et al. 2009 find less than $10 \%$ of the Lyman- $\alpha$ photons to be generated by gravitational cooling, but since they are primarily emitted in the galactic outskirts and not in dense, dusty, star forming clumps, they could contribute significantly to the escape fraction. On the other hand, Dijkstra et al. 2006 show that the expected spectrum from Lyman- $\alpha$ photons generated by gravitational cooling is preferentially blue, making absorption of this flux in the IGM more probable. However, it is clear that including dust has major

1 For technical details, see the description at http://www.multidark.org/MultiDark/Help?page=databases/mdpl/database. A paper describing the details of the simulation is in preparation. 
impact on the observed Lyman- $\alpha$ properties in an evolved population of emitters, but including dust is also not a straight-forward task in cosmological simulations. Most of the dust is situated in dense, cold clumps. These clouds are typically not resolved by cosmological simulations, leading to the necessity of a subgrid model to fully capture its effects. The same difficulty arises for studies of e.g. the inclination effect in such large-scale simulations.

The inclination dependency that we found in the simulations in Behrens \& Niemeyer 2013 partly motivated the second publication, Behrens et al. 2014. In this work, we presented three families of simplified models of LAEs, that in contrast to most of the models in the literature (see chapter 3) feature significant anisotropies in density and velocity fields. The common property of the models we presented is the existence of lines of sights with lower or zero optical depth along one principal direction. In many cases, this leads to a dependency of the flux on the inclination an observer has, i.e. more Lyman- $\alpha$ photons escape towards directions in which the optical depth is reduced. This, in turn, results in observers at different inclinations seeing different EWs. We find that in our simplified models, the observed EW can be boosted by a factor of a few compared to the isotropic case. This could partly explain the observation that many LAEs show EWs too high to be explained by the galaxy spectra expected from star formation activity alone. We also find that in many cases the spectrum is modified for observers along lines of sights with reduced optical depth: In particular, those observers see the unscattered input spectrum or, depending on the spectral resolution of the observations, an excess flux near the line center. These findings are potentially helpful in finding Lyman continuum leakers, i.e. galaxies that leak ionizing radiation: Since Lyman continuum is known to have a scattering cross section three orders of magnitude lower than Lyman- $\alpha$ photons (e.g. Verhamme et al. 2014), the spectral signature of a cavity along the line of sight can help to select targets for which it is probable to also find Lyman continuum.

The simplified models of the ISM can be seen as complementary to the cosmological simulation in Behrens \& Niemeyer 2013, since they present a more detailed model of the transport in the ISM that is not resolved in Behrens \& Niemeyer 2013, but ignore effects from the intervening IGM between the LAEs and the observer. The IGM would reduce the observed flux, primarily in the blue part of the spectrum. Additionally, the models are still very symmetric and homogeneous compared to the fuzzy, chaotic structure observed in real galaxies. However, it is the purpose of such simplified models to be crude approximations that still capture features of a complex 
physical reality, and even far simpler models of LAEs like the shell model (see section 3.1) have been proven partially useful already. In this context, an open task might be to compare the observational effects of the different proposed anisotropic models by Zheng \& Wallace 2014, Verhamme et al. 2014, and others. While the observational prospects for Lyman continuum have already been mentioned, we additionally note that the models and their inclination dependency can be a basis for subgrid modeling of LAEs and for quantifying a possible tidal alignment effect.

In Behrens \& Braun 2014, we quantify the effects of anisotropic emitters in a more realistic setup by using a simulation of an isolated disk galaxy run by Braun et al. 2014, including detailed physics of star formation, turbulence, feedback and the ISM. The resolution of this simulation enables us to probe the effect of the ISM and its clumpy, inhomogeneous structure on the observed Lyman- $\alpha$ properties. As expected, we find a strong inclination dependency of the flux arising from the disk, similar to Verhamme et al. 2014. Additionally, we find temporal variations of the flux, i.e. the used snapshots of the disk galaxy at 1/1.5/2 Gyr after initialization of the disk show large differences in their total escape fractions and EW distribution. We show that these differences arise from the small-scale structure of the ISM. In particular, supernovae-driven, evacuated bubbles in the disk can enhance escape due to the reduced optical depth and the emergence of optically-thin lines of sight. Since the bubbles are short-lived, we conclude that this variability will take place on very short timescales, comparable to the timescale of the self-regulated star formation $(\sim$ few 10 Myr). This mechanism is very robust against changes in the dust content. However, we find that the transmitting bubbles partly inhabit dense star clusters in which the stars dominate the gravitational potential. While in the less massive clusters, star formation is suppressed by the supernova feedback, the massive clusters form stars iteratively. It is not sure if such massive clusters exist in forming spiral galaxies or if they are related to shortcomings of the employed physical model of Braun et al. 2014. Observational data with sufficient resolution to answer this question is not available to date.

Apart from these uncertainties, it is obvious that the simulated disk galaxy lacks a cosmological background and a cosmological environment, since it is isolated. For example, tidal forces from other galaxies could strongly influence its morphology and therefore its emissivity/transmissivity. Accretion of material from the CGM on the other hand would also affect the local density and velocity structure, changing the Lyman- $\alpha$ properties. In particular, it is well known that the blue part of the 
spectrum will be suppressed by infalling material (see section 3.1). Additionally, the influence of the IGM on the transmitted flux has not been included in our calculations. It therefore remains a task for the future to study Lyman- $\alpha$ properties and the inclination effect in a full-fledged cosmological simulation with high resolution. In particular, to obtain robust statements, studies of individual emitters need to be complemented with statistics on a larger number of simulated emitters with different initial conditions.

We have supplemented these findings in chapter 2 and 3 by summarizing the work that has been done on contamination by radiative transfer effects and LAE modeling in the literature. Although a lot of progress has been made, in particular in deriving a formalism to describe contamination from large-scale structure (Hirata 2009; Wyithe \& Dijkstra 2011) and in understanding at least some Lyman- $\alpha$ line profiles (e.g. Verhamme et al. 2006), a lot of open questions remain: What is the full explanation for the observed high EWs in Lyman- $\alpha$ galaxies? What is the origin of the diversity of observed line profiles? How important is gravitational cooling for Lyman- $\alpha$ emission? The vast observational data that will be delivered by HETDEX, the MUSE instrument, and Subaru HyperSuprime Cam in the next years will be helpful to address these and other questions. However, complementary simulations will remain an important tool. 


\section{Bibliography}

Adams, J. J., G. A. Blanc, G. J. Hill, K. Gebhardt, N. Drory, L. Hao, R. Bender, J. Byun, R. Ciardullo, M. E. Cornell, S. L. Finkelstein, A. Fry, E. Gawiser, C. Gronwall, U. Hopp, D. Jeong, A. Kelz, R. Kelzenberg, E. Komatsu, P. J. MacQueen, J. Murphy, P. S. Odoms, M. Roth, D. P. Schneider, J. R. Tufts, \& C. P. Wilkinson (2011): 'The HETDEX Pilot Survey. I. Survey Design, Performance, and Catalog of Emission-line Galaxies'. ApJS, vol. 192,5 : p. 5.

Adams, T. F. (1972): 'The Escape of Resonance-Line Radiation from Extremely Opaque Media'. ApJ, vol. 174: p. 439

Ahn, Sang-Hyeon, Hee-Won Lee, \& Hyung-Mok Lee (2000): 'Lyman alpha Transfer in a thick, dusty, and static medium'. en. Journal of Korean Astronomical Society, vol. 33: pp. 29-36.

Ahn, S.-H., H.-W. Lee, \& H. M. Lee (2003): 'P Cygni type Ly $\alpha$ from starburst galaxies'. MNRAS, vol. 340: pp. 863-869.

Ajiki, M., Y. Taniguchi, S. S. Fujita, Y. Shioya, T. Nagao, T. Murayama, S. Yamada, K. Umeda, \& Y. Komiyama (2003): 'A Subaru Search for Ly $\alpha$ Emitters at Redshift 5.7'. $A J$, vol. 126: pp. 2091-2107.

Almgren, A. S., J. B. Bell, M. J. Lijewski, Z. Lukić, \& E. Van Andel (2013): 'Nyx: A Massively Parallel AMR Code for Computational Cosmology'. ApJ, vol. 765, 39: p. 39. Aragon-Calvo, M. A., R. van de Weygaert, B. J. T. Jones, \& J. M. van der Hulst (2007): 'Spin Alignment of Dark Matter Halos in Filaments and Walls'. ApJ, vol. 655: pp. L5-L8. Barger, A. J., L. L. Cowie, \& I. G. B. Wold (2012): 'A Flux-limited Sample of z 1 Ly $\alpha$ Emitting Galaxies in the Chandra Deep Field South'. ApJ, vol. 749, 106: p. 106.

Barnes, L. A., T. Garel, \& G. G. Kacprzak (2014): 'Lyman Alpha and MgII as Probes of Galaxies and their Environments'. ArXiv e-prints, vol.

Barnes, L. A., M. G. Haehnelt, E. Tescari, \& M. Viel (2011): 'Galactic winds and extended Ly $\alpha$ emission from the host galaxies of high column density quasi-stellar object absorption systems'. MNRAS, vol. 416: pp. 1723-1738. 
Becker, R. H., X. Fan, R. L. White, M. A. Strauss, V. K. Narayanan, R. H. Lupton, J. E. Gunn, J. Annis, N. A. Bahcall, J. Brinkmann, A. J. Connolly, I. Csabai, P. C. Czarapata, M. Doi, T. M. Heckman, G. S. Hennessy, Ž. Ivezić, G. R. Knapp, D. Q. Lamb, T. A. McKay, J. A. Munn, T. Nash, R. Nichol, J. R. Pier, G. T. Richards, D. P. Schneider, C. Stoughton, A. S. Szalay, A. R. Thakar, \& D. G. York (2001): 'Evidence for Reionization at $\mathrm{z}^{\sim} 6$ : Detection of a Gunn-Peterson Trough in a $\mathrm{z}=6.28$ Quasar'. $A J$, vol. 122: pp. 2850-2857.

Behrens, C. (2011): 'Numerische Simulation des Transports von Lyman-Alpha-Strahlung auf kosmologischen Skalen'. MA thesis. University of G'ottingen.

Behrens, C. \& H. Braun (2014). A\& A, vol. accepted.

Behrens, C., M. Dijkstra, \& J. C. Niemeyer (2014): 'Beamed Ly $\alpha$ emission through outflow-driven cavities'. $A \& \& A$, vol. 563: A77.

Behrens, C. \& J. C. Niemeyer (2013): 'Effects of Lyman-alpha scattering in the IGM on clustering statistics of Lyman-alpha emitters'. ApJ, vol. 556: A5.

Benson, A. J. (2012): 'G ALACTICUS: A semi-analytic model of galaxy formation'. New A, vol. 17: pp. 175-197.

Bernstein, G. M. (2009): 'Comprehensive Two-Point Analyses of Weak Gravitational Lensing Surveys'. ApJ, vol. 695: pp. 652-665.

Birnboim, Y. \& A. Dekel (2003): 'Virial shocks in galactic haloes?' MNRAS, vol. 345: pp. 349-364.

Bond, N. A., E. Gawiser, L. Guaita, N. Padilla, C. Gronwall, R. Ciardullo, \& K. Lai (2012): 'Evolution in the Continuum Morphological Properties of Ly $\alpha$-emitting Galaxies from z $=3.1$ to $\mathrm{z}=2.1^{\prime}$. ApJ, vol. $753,95:$ p. 95 .

Braun, H., W. Schmidt, J. C. Niemeyer, \& A. S. Almgren (2014): 'Large-eddy simulations of isolated disc galaxies with thermal and turbulent feedback'. MNRAS, vol. 442: pp. 34073426 .

Cantalupo, S., F. Arrigoni-Battaia, J. X. Prochaska, J. F. Hennawi, \& P. Madau (2014): 'A cosmic web filament revealed in Lyman- $\alpha$ emission around a luminous high-redshift quasar'. Nature, vol. 506: pp. 63-66.

Cassata, P., O. Le Fèvre, B. Garilli, D. Maccagni, V. Le Brun, M. Scodeggio, L. Tresse, O. Ilbert, G. Zamorani, O. Cucciati, T. Contini, R. Bielby, Y. Mellier, H. J. McCracken, A. Pollo, A. Zanichelli, S. Bardelli, A. Cappi, L. Pozzetti, D. Vergani, \& E. Zucca (2011): 'The VIMOS VLT Deep Survey: star formation rate density of Ly $\alpha$ emitters from a sample of 217 galaxies with spectroscopic redshifts $2<\mathrm{z}<6.6^{\prime}$. A\&A, vol. 525, A143: A143. 
Cen, R. \& Z. Zheng (2013): 'The nature of Ly $\alpha$ blobs: powered by extreme starbursts'. ApJ, vol. 775(2): p. 112.

Chakraborti, S. \& A. Ray (2011): 'An Expanding Neutral Hydrogen Supershell Evacuated by Multiple Supernovae in M101'. ApJ, vol. 728, 24: p. 24.

Chonis, T. S., G. A. Blanc, G. J. Hill, J. J. Adams, S. L. Finkelstein, K. Gebhardt, J. A. Kollmeier, R. Ciardullo, N. Drory, C. Gronwall, A. Hagen, R. A. Overzier, M. Song, \& G. R. Zeimann (2013): 'The Spectrally Resolved Ly $\alpha$ Emission of Three Ly $\alpha$-selected Field Galaxies at z $\sim 2.4$ from the HETDEX Pilot Survey'. ApJ, vol. 775, 99: p. 99.

Ciardullo, R., C. Gronwall, G. Blanc, S. Finkelstein, E. Gawiser, K. Gebhardt, \& HETDEX Collaboration (2012): 'HETDEX and the Evolution of The Physical Properties of LymanAlpha Emitting Galaxies'. American Astronomical Society Meeting Abstracts \#220. Vol. 220. American Astronomical Society Meeting Abstracts: p. 429.07.

Codis, S., R. Gavazzi, Y. Dubois, C. Pichon, K. Benabed, V. Desjacques, D. Pogosyan, J. Devriendt, \& A. Slyz (2014): 'Intrinsic alignment of simulated galaxies in the cosmic web: implications for weak lensing surveys'. ArXiv e-prints, vol.

Codis, S., C. Pichon, J. Devriendt, A. Slyz, D. Pogosyan, Y. Dubois, \& T. Sousbie (2012): 'Connecting the cosmic web to the spin of dark haloes: implications for galaxy formation'. MNRAS, vol. 427: pp. 3320-3336.

Dawson, S., J. E. Rhoads, S. Malhotra, D. Stern, A. Dey, H. Spinrad, B. T. Jannuzi, J. Wang, \& E. Landes (2004): 'Spectroscopic Properties of the $\mathrm{z} \sim 4.5$ Ly $\alpha$ Emitters'. ApJ, vol. 617: pp. 707-717.

Dawson, S., J. E. Rhoads, S. Malhotra, D. Stern, J. Wang, A. Dey, H. Spinrad, \& B. T. Jannuzi (2007): 'A Luminosity Function of Ly $\alpha$-emitting Galaxies at z 4.5'. ApJ, vol. 671: pp. $1227-1240$

Dayal, P., A. Maselli, \& A. Ferrara (2011): 'The visibility of Lyman $\alpha$ emitters during reionization'. MNRAS, vol. 410: pp. 830-843.

Dijkstra, M. (2010): 'Observational Signatures of Lyman Alpha Emission from Early Galaxy Formation.' Cosmic Radiation Fields: Sources in the early Universe (CRF 2010). Ed. by Raue, M., T. Kneiske, D. Horns, D. Elsaesser, \& P. Hauschildt: p. 35.

- (2014): 'Lyman Alpha Emitting Galaxies as a Probe of Reionization'. ArXiv e-prints, vol.

Dijkstra, M., Z. Haiman, \& M. Spaans (2006): 'Lyman Alpha Radiation From Collapsing Protogalaxies I: Characteristics of the Emergent Spectrum'. ApJ, vol. 649(1): pp. 14-36. Dijkstra, M. \& J. Wyithe (2010): 'Seeing through the trough: outflows and the detectability of Ly $\alpha$ emission from the first galaxies'. MNRAS, vol. 408(1): pp. 352-361. 
Dijkstra, Mark, Andrei Mesinger, \& J. Stuart B. Wyithe (2011): 'The detectability of Ly $\alpha$ emission from galaxies during the epoch of reionization'. MNRAS, vol. 414(3): pp. 2139-2147.

Djorgovski, S., H. Spinrad, P. McCarthy, \& M. A. Strauss (1985): 'Discovery of a probable galaxy with a redshift of 3.218'. ApJ, vol. 299: pp. L1-L5.

Dressler, A., C. L. Martin, A. Henry, M. Sawicki, \& P. McCarthy (2011): 'Detections of Faint Ly $\alpha$ Emitters at z $=5.7$ : Galaxy Building Blocks and Engines of Reionization'. ApJ, vol. 740, 71: p. 71.

Dubois, Y. et al. (2014): 'Dancing in the dark: galactic properties trace spin swings along the cosmic web'. ArXiv e-prints, vol.

Duval, Florent, Daniel Schaerer, Göran Östlin, \& Peter Laursen (2014): 'Lyman $\alpha$ line and continuum radiative transfer in a clumpy interstellar medium'. $A \& A$, vol. 562: A52.

Faltenbacher, A., C. Li, S. D. M. White, Y.-P. Jing, Shu-DeMao, \& J. Wang (2009): 'Alignment between galaxies and large-scale structure'. Research in Astronomy and Astrophysics, vol. 9: pp. 41-58.

Finkelstein, S. L., J. E. Rhoads, S. Malhotra, N. Grogin, \& J. Wang (2008): 'Effects of Dust Geometry in Ly $\alpha$ Galaxies at $\mathrm{z}=4.4^{\prime}$. ApJ, vol. 678: pp. 655-668.

Furlanetto, S. R., J. Schaye, V. Springel, \& L. Hernquist (2005): 'Ly $\alpha$ Emission from Structure Formation'. ApJ, vol. 622: pp. 7-27.

Gawiser, E., H. Francke, K. Lai, K. Schawinski, C. Gronwall, R. Ciardullo, R. Quadri, A. Orsi, L. F. Barrientos, G. A. Blanc, G. Fazio, J. J. Feldmeier, J.-s. Huang, L. Infante, P. Lira, N. Padilla, E. N. Taylor, E. Treister, C. M. Urry, P. G. van Dokkum, \& S. N. Virani (2007): 'Ly $\alpha$-Emitting Galaxies at $\mathrm{z}=3.1$ : L* Progenitors Experiencing Rapid Star Formation'. ApJ, vol. 671: pp. 278-284.

Gawiser, E., P. G. van Dokkum, C. Gronwall, R. Ciardullo, G. A. Blanc, F. J. Castander, J. Feldmeier, H. Francke, M. Franx, L. Haberzettl, D. Herrera, T. Hickey, L. Infante, P. Lira, J. Maza, R. Quadri, A. Richardson, K. Schawinski, M. Schirmer, E. N. Taylor, E. Treister, C. M. Urry, \& S. N. Virani (2006): 'The Physical Nature of Ly $\alpha$-emitting Galaxies at $\mathrm{z}=3.1^{\prime}$. ApJ, vol. 642: pp. L13-L16.

Greig, B., E. Komatsu, \& J. S. B. Wyithe (2013): 'Cosmology from clustering of Ly $\alpha$ galaxies: breaking non-gravitational Ly $\alpha$ radiative transfer degeneracies using the bispectrum'. MNRAS, vol. 431: pp. 1777-1794.

Gronke, M. \& M. Dijkstra (2014): 'Directional Ly $\alpha$ equivalent boosting - I. Spherically symmetric distributions of clumps'. MNRAS, vol. 444: pp. 1095-1103.

Gronwall, C., R. Ciardullo, T. Hickey, E. Gawiser, J. J. Feldmeier, P. G. van Dokkum, C. M. Urry, D. Herrera, B. D. Lehmer, L. Infante, A. Orsi, D. Marchesini, G. A. Blanc, 
H. Francke, P. Lira, \& E. Treister (2007): 'Ly $\alpha$ Emission-Line Galaxies at $\mathrm{z}=3.1$ in the Extended Chandra Deep Field-South'. ApJ, vol. 667: pp. 79-91.

Grove, L. F., J. P. U. Fynbo, C. Ledoux, M. Limousin, P. Møller, K. K. Nilsson, \& B. Thomsen (2009): 'The Building the Bridge survey for $\mathrm{z}=3$ Ly $\alpha$ emitting galaxies. II. Completion of the survey'. A\&\&A, vol. 497: pp. 689-702.

Guaita, L., E. Gawiser, N. Padilla, H. Francke, N. A. Bond, C. Gronwall, R. Ciardullo, J. J. Feldmeier, S. Sinawa, G. A. Blanc, \& S. Virani (2010): 'Ly $\alpha$-emitting Galaxies at $\mathrm{z}=2.1$ in ECDF-S: Building Blocks of Typical Present-day Galaxies?' ApJ, vol. 714: pp. 255-269.

Gunn, J. E. \& B. A. Peterson (1965): 'On the Density of Neutral Hydrogen in Intergalactic Space.' ApJ, vol. 142: pp. 1633-1641.

Hagen, A., R. Ciardullo, C. Gronwall, V. Acquaviva, J. Bridge, G. R. Zeimann, G. A. Blanc, N. A. Bond, S. L. Finkelstein, M. Song, E. Gawiser, D. B. Fox, H. Gebhardt, A. I. Malz, D. P. Schneider, N. Drory, K. Gebhardt, \& G. J. Hill (2014): 'Spectral Energy Distribution Fitting of HETDEX Pilot Survey Ly $\alpha$ Emitters in COSMOS and GOODS-N'. ApJ, vol. 786, 59: p. 59.

Hahn, O., R. Teyssier, \& C. M. Carollo (2010): 'The large-scale orientations of disc galaxies'. MNRAS, vol. 405: pp. 274-290.

Haiman, Z. \& M. Spaans (1999): 'Models for Dusty Ly $\alpha$ Emitters at High Redshift'. ApJ, vol. 518: pp. 138-144.

Hansen, M. \& S. P. Oh (2006): 'Lyman Alpha Radiative Transfer in a Multi-Phase Medium'. MNRAS, vol. 367: pp. 979-1002.

Harrington, J. P. (1973): 'The scattering of resonance-line radiation in the limit of large optical depth'. MNRAS, vol. 162: p. 43.

Hayashino, T., Y. Matsuda, H. Tamura, R. Yamauchi, T. Yamada, M. Ajiki, S. S. Fujita, T. Murayama, T. Nagao, K. Ohta, S. Okamura, M. Ouchi, K. Shimasaku, Y. Shioya, \& Y. Taniguchi (2004): 'Large-Scale Structure of Emission-Line Galaxies at $\mathrm{z}=3.1$ '. $A J$, vol. 128: pp. 2073-2079.

Hayes, M., G. Östlin, D. Schaerer, J. M. Mas-Hesse, C. Leitherer, H. Atek, D. Kunth, A. Verhamme, S. de Barros, \& J. Melinder (2010): 'Escape of about five per cent of Lyman- $\alpha$ photons from high-redshift star-forming galaxies'. Nature, vol. 464: pp. 562-565.

Hayes, M., C. Scarlata, \& B. Siana (2011): 'Central powering of the largest Lyman- $\alpha$ nebula is revealed by polarized radiation'. Nature, vol. 476: pp. 304-307.

Heiles, C. (1979): 'H I shells and supershells'. ApJ, vol. 229: pp. 533-537.

- (1984): 'H I shells, supershells, shell-like objects, and 'worms". ApJS, vol. 55: pp. 585-595. 
Henault, F., R. Bacon, C. Bonneville, D. Boudon, R. L. Davies, P. Ferruit, G. F. Gilmore, O. LeFevre, J. Lemonnier, S. Lilly, S. Simon L. Morris, E. Prieto, M. Steinmetz, \& P. T. de Zeeuw (2003): 'MUSE: a second-generation integral-field spectrograph for the VLT'. Astronomical Telescopes and Instrumentation. Ed. by Iye, Masanori \& Alan F. M. Moorwood. International Society for Optics and Photonics: pp. 1096-1107.

Hennebelle, P. \& E. Falgarone (2012): 'Turbulent molecular clouds'. A\&A Rev. Vol. 20, 55: p. 55 .

Henyey, L. \& J. Greenstein (1941): 'Diffuse radiation in the Galaxy'. ApJ, vol. 93: pp. 70-83.

Hibon, P., J.-G. Cuby, J. Willis, B. Clément, C. Lidman, S. Arnouts, J.-P. Kneib, C. J. Willott, C. Marmo, \& H. McCracken (2010): 'Limits on the luminosity function of Ly $\alpha$ emitters at $\mathrm{z}=7.7$. A\& $A$, vol. 515, A97: A97.

Hibon, P., N. Kashikawa, C. Willott, M. Iye, \& T. Shibuya (2012): 'Search for z $~ 7$ Ly $\alpha$ Emitters with the Suprime-Cam at the Subaru Telescope'. ApJ, vol. 744, 89: p. 89.

Hill, G. J., K. Gebhardt, E. Komatsu, N. Drory, P. J. MacQueen, J. Adams, G. A. Blanc, R. Koehler, M. Rafal, M. M. Roth, A. Kelz, C. Gronwall, R. Ciardullo, \& D. P. Schneider (2008): 'The Hobby-Eberly Telescope Dark Energy Experiment (HETDEX): Description and Early Pilot Survey Results'. Panoramic Views of Galaxy Formation and Evolution. Ed. by Kodama, T., T. Yamada, \& K. Aoki. Vol. 399. Astronomical Society of the Pacific Conference Series: p. 115.

Hirata, C. M. (2009): 'Tidal alignments as a contaminant of redshift space distortions'. MNRAS, vol. 399(2): pp. 1074-1087.

Hirata, C. M., R. Mandelbaum, M. Ishak, U. Seljak, R. Nichol, K. A. Pimbblet, N. P. Ross, \& D. Wake (2007): 'Intrinsic galaxy alignments from the 2SLAQ and SDSS surveys: luminosity and redshift scalings and implications for weak lensing surveys'. MNRAS, vol. 381: pp. 1197-1218.

Hu, E. M., L. L. Cowie, A. J. Barger, P. Capak, Y. Kakazu, \& L. Trouille (2010): 'An Atlas of $\mathrm{z}=5.7$ and $\mathrm{z}=6.5$ Ly $\alpha$ Emitters'. ApJ, vol. 725: pp. 394-423.

Hu, E. M., L. L. Cowie, P. Capak, R. G. McMahon, T. Hayashino, \& Y. Komiyama (2004): 'The Luminosity Function of Ly $\alpha$ Emitters at Redshift z 5.7 '. AJ, vol. 127: pp. 563-575. Hu, E. M., L. L. Cowie, \& R. G. McMahon (1998): 'The Density of Ly $\alpha$ Emitters at Very High Redshift'. ApJ, vol. 502: pp. L99-L103.

Hu, E. M., L. L. Cowie, R. G. McMahon, P. Capak, F. Iwamuro, J.-P. Kneib, T. Maihara, \& K. Motohara (2002): 'A Redshift $\mathrm{z}=6.56$ Galaxy behind the Cluster Abell 370'. ApJ, vol. 568: pp. L75-L79.

Hu, E. M. \& R. G. McMahon (1996): 'Detection of Lyman- $\alpha$-emitting galaxies at redshift 4.55'. Nature, vol. 382: pp. 231-233. 
Inoue, A. K. (2003): 'Evolution of Dust-to-Metal Ratio in Galaxies'. PASJ, vol. 55(5): pp. 901-909.

Kaiser, N. (1984): 'On the spatial correlations of Abell clusters'. ApJ, vol. 284: pp. L9-L12. - (1987): 'Clustering in real space and in redshift space'. MNRAS, vol. 227: pp. 1-21.

Kashikawa, N., K. Shimasaku, M. A. Malkan, M. Doi, Y. Matsuda, M. Ouchi, Y. Taniguchi, C. Ly, T. Nagao, M. Iye, K. Motohara, T. Murayama, K. Murozono, K. Nariai, K. Ohta, S. Okamura, T. Sasaki, Y. Shioya, \& M. Umemura (2006): 'The End of the Reionization Epoch Probed by Ly $\alpha$ Emitters at $\mathrm{z}=6.5$ in the Subaru Deep Field'. ApJ, vol. 648: pp. $7-22$.

Kodaira, K. et al. (2003): 'The Discovery of Two Lyman $\alpha$ Emitters beyond Redshift 6 in the Subaru Deep Field'. PASJ, vol. 55: pp. L17-L21.

Krug, H. B., S. Veilleux, V. Tilvi, S. Malhotra, J. Rhoads, P. Hibon, R. Swaters, R. Probst, A. Dey, M. Dickinson, \& B. T. Jannuzi (2012): 'Searching for z $~ 7.7$ Ly $\alpha$ Emitters in the COSMOS Field with NEWFIRM'. ApJ, vol. 745, 122: p. 122.

Kudritzki, R.-P., R. H. Méndez, J. J. Feldmeier, R. Ciardullo, G. H. Jacoby, K. C. Freeman, M. Arnaboldi, M. Capaccioli, O. Gerhard, \& H. C. Ford (2000): 'Discovery of Nine Ly $\alpha$ Emitters at Redshift z $\sim 3.1$ Using Narrowband Imaging and VLT Spectroscopy'. ApJ, vol. 536: pp. 19-30.

Kulas, Kristin R., Alice E. Shapley, Juna A. Kollmeier, Zheng Zheng, Charles C. Steidel, \& Kevin N. Hainline (2012): 'The Kinematics of Multiple-peaked Ly $\alpha$ Emission in Star-forming Galaxies at z $\sim 2-3$ '. ApJ, vol. 745(1): p. 33 .

Kurk, J. D., A. Cimatti, S. di Serego Alighieri, J. Vernet, E. Daddi, A. Ferrara, \& B. Ciardi (2004): 'A Lyman $\alpha$ emitter at $\mathrm{z}=6.5$ found with slitless spectroscopy'. $A \mathscr{E} A$, vol. 422 : pp. L13-L17.

Lai, K., J.-S. Huang, G. Fazio, E. Gawiser, R. Ciardullo, M. Damen, M. Franx, C. Gronwall, I. Labbe, G. Magdis, \& P. van Dokkum (2008): 'Spitzer Constraints on the Stellar Populations of Ly $\alpha$-Emitting Galaxies at $\mathrm{z}=3.1^{\prime}$. ApJ, vol. 674: pp. 70-74.

Laursen, P. (2010): 'Lyman Alpha radiative transfer in the high-redshift, dusty Universe'. ArXiv e-prints, vol.

Laursen, P., F. Duval, \& G. Östlin (2013): 'On the (Non-)Enhancement of the Ly $\alpha$ Equivalent Width by a Multiphase Interstellar Medium'. ApJ, vol. 766, 124: p. 124.

Laursen, P. \& J. Sommer-Larsen (2007): 'Ly $\alpha$ Resonant Scattering in Young Galaxies: Predictions from Cosmological Simulations'. ApJ, vol. 657(2): pp. L69-L72.

Laursen, P., J. Sommer-Larsen, \& A. C. Andersen (2009): 'Ly $\alpha$ Radiative Transfer with Dust: Escape Fractions from Simulated High-Redshift Galaxies'. ApJ, vol. 704: pp. 16401656. 
Laursen, P., J. Sommer-Larsen, \& A. O. Razoumov (2010): 'Intergalactic Transmission and its Impact on the Ly $\alpha$ Line'. ApJ, vol. 728(1): p. 5.

Libeskind, N. I., Y. Hoffman, J. Forero-Romero, S. Gottlöber, A. Knebe, M. Steinmetz, \& A. Klypin (2013): 'The velocity shear tensor: tracer of halo alignment'. MNRAS, vol. 428: pp. 2489-2499.

Mandelbaum, R., C. M. Hirata, M. Ishak, U. Seljak, \& J. Brinkmann (2006): 'Detection of large-scale intrinsic ellipticity-density correlation from the Sloan Digital Sky Survey and implications for weak lensing surveys'. MNRAS, vol. 367: pp. 611-626.

Matsuda, Y., T. Yamada, T. Hayashino, R. Yamauchi, Y. Nakamura, N. Morimoto, M. Ouchi, Y. Ono, K. Kousai, E. Nakamura, M. Horie, T. Fujii, M. Umemura, \& M. Mori (2011): 'The Subaru Ly $\alpha$ blob survey: a sample of 100-kpc Ly $\alpha$ blobs at $\mathrm{z}=3$ '. MNRAS, vol. 410: pp. L13-L17.

McLinden, E. M., S. L. Finkelstein, J. E. Rhoads, S. Malhotra, P. Hibon, M. L. A. Richardson, G. Cresci, A. Quirrenbach, A. Pasquali, F. Bian, X. Fan, \& C. E. Woodward (2011): 'First Spectroscopic Measurements of [O III] Emission from Ly $\alpha$ Selected Field Galaxies at $\mathrm{z} \sim 3.1^{\prime}$. ApJ, vol. 730, 136: p. 136 .

Nakajima, K., M. Ouchi, K. Shimasaku, Y. Ono, J. C. Lee, S. Foucaud, C. Ly, D. A. Dale, S. Salim, R. Finn, O. Almaini, \& S. Okamura (2012): 'Average Metallicity and Star Formation Rate of Ly $\alpha$ Emitters Probed by a Triple Narrowband Survey'. ApJ, vol. 745, 12: p. 12.

Neufeld, D. A. (1990): 'The transfer of resonance-line radiation in static astrophysical media'. ApJ, vol. 350: pp. 216-241.

- (1991): 'The escape of Lyman-alpha radiation from a multiphase interstellar medium'. ApJ, vol. 370: p. L85.

Nilsson, K. K., P. Møller, O. Möller, J. P. U. Fynbo, M. J. Michałowski, D. Watson, C. Ledoux, P. Rosati, K. Pedersen, \& L. F. Grove (2007): 'A multi-wavelength study of z = 3.15 Lyman- $\alpha$ emitters in the GOODS South Field'. A\&A, vol. 471: pp. 71-82.

Nilsson, K. K., C. Tapken, P. Moller, W. Freudling, J. P. U. Fynbo, K. Meisenheimer, P. Laursen, \& G. Östlin (2009): 'Evolution in the properties of Lyman- $\alpha$ emitters from redshifts $\mathrm{z} \sim 3$ to $\mathrm{z} \sim 2$ '. A\&A, vol. 498: pp. 13-23.

Ocvirk, P., C. Pichon, \& R. Teyssier (2008): 'Bimodal gas accretion in the HorizonMareNostrum galaxy formation simulation'. MNRAS, vol. 390(4): p. 15.

Ouchi, M., K. Shimasaku, M. Akiyama, C. Simpson, T. Saito, Y. Ueda, H. Furusawa, K. Sekiguchi, T. Yamada, T. Kodama, N. Kashikawa, S. Okamura, M. Iye, T. Takata, M. Yoshida, \& M. Yoshida (2008): 'The Subaru/XMM-Newton Deep Survey (SXDS). IV. 
Evolution of Ly $\alpha$ Emitters from $\mathrm{z}=3.1$ to 5.7 in the $1 \mathrm{deg}^{2}$ Field: Luminosity Functions and AGN'. ApJS, vol. 176: pp. 301-330.

Ouchi, M., K. Shimasaku, H. Furusawa, M. Miyazaki, M. Doi, M. Hamabe, T. Hayashino, M. Kimura, K. Kodaira, Y. Komiyama, Y. Matsuda, S. Miyazaki, F. Nakata, S. Okamura, M. Sekiguchi, Y. Shioya, H. Tamura, Y. Taniguchi, M. Yagi, \& N. Yasuda (2003): 'Subaru Deep Survey. II. Luminosity Functions and Clustering Properties of Ly $\alpha$ Emitters at $\mathrm{z}=4.86$ in the Subaru Deep Field'. ApJ, vol. 582: pp. 60-68.

Ouchi, M., K. Shimasaku, H. Furusawa, T. Saito, M. Yoshida, M. Akiyama, Y. Ono, T. Yamada, K. Ota, N. Kashikawa, M. Iye, T. Kodama, S. Okamura, C. Simpson, \& M. Yoshida (2010): 'Statistics of 207 Ly $\alpha$ Emitters at a Redshift Near 7: Constraints on Reionization and Galaxy Formation Models'. ApJ, vol. 723: pp. 869-894.

Partridge, R. B. \& P. J. E. Peebles (1967): 'Are Young Galaxies Visible?' ApJ, vol. 147: p. 868.

Pascarelle, S. M., R. A. Windhorst, S. P. Driver, E. J. Ostrander, \& W. C. Keel (1996): 'The Serendipitous Discovery of a Group or Cluster of Young Galaxies at z $=2.40$ in Deep Hubble Space Telescope WFPC2 Images'. ApJ, vol. 456: p. L21.

Pascarelle, S. M., R. A. Windhorst, \& W. C. Keel (1998): 'Compact Lyalpha-emitting Candidates at $\mathrm{Z} \sim 2.4$ in Deep Medium-Band Hubble Space Telescope WFPC2 Images'. $A J$, vol. 116: pp. 2659-2666.

Press, William H., Saul A. Teukolsky, William T. Vetterling, \& Brian P. Flannery (2007): Numerical Recipes 3rd Edition: The Art of Scientific Computing. 3rd ed. New York, NY, USA: Cambridge University Press.

Pritchet, C. J. (1994): 'The search for primeval galaxies'. PASP, vol. 106: pp. 1052-1067. Rauch, M., M. Haehnelt, A. Bunker, G. Becker, F. Marleau, J. Graham, S. Cristiani, M. Jarvis, C. Lacey, S. Morris, C. Peroux, H. Roettgering, \& T. Theuns (2008): 'Faint Ly $\alpha$ Emitters, Star-forming Galaxies, and Damped Ly $\alpha$ Systems'. Panoramic Views of Galaxy Formation and Evolution. Ed. by Kodama, T., T. Yamada, \& K. Aoki. Vol. 399. Astronomical Society of the Pacific Conference Series: p. 59.

Rhoads, J. E., A. Dey, S. Malhotra, D. Stern, H. Spinrad, B. T. Jannuzi, S. Dawson, M. J. I. Brown, \& E. Landes (2003): 'Spectroscopic Confirmation of Three Redshift z $\sim 5.7$ Ly $\alpha$ Emitters from the Large-Area Lyman Alpha Survey'. AJ, vol. 125: pp. 1006-1013.

Rhoads, J. E. \& S. Malhotra (2001): 'Ly $\alpha$ Emitters at Redshift $\mathrm{z}=5.7$ '. ApJ, vol. 563: pp. L5-L9.

Rhoads, J. E., S. Malhotra, A. Dey, B. T. Jannuzi, D. Stern, \& H. Spinrad (2001): 'The Large Area Lyman Alpha Survey.' The New Era of Wide Field Astronomy. Ed. by 
Clowes, R., A. Adamson, \& G. Bromage. Vol. 232. Astronomical Society of the Pacific Conference Series: p. 196.

Rhoads, J. E., C. Xu, S. Dawson, A. Dey, S. Malhotra, J. Wang, B. T. Jannuzi, H. Spinrad, \& D. Stern (2004): 'A Luminous Ly $\alpha$-emitting Galaxy at Redshift $\mathrm{z}=6.535$ : Discovery and Spectroscopic Confirmation'. ApJ, vol. 611: pp. 59-67.

Richling, S. (2003): 'Resonance line transfer in clumpy media'. MNRAS, vol. 344: pp. 553561.

Rubin, K. H. R., J. X. Prochaska, D. C. Koo, A. C. Phillips, C. L. Martin, \& L. O. Winstrom (2013): 'Evidence for Ubiquitous Collimated Galactic-Scale Outflows along the Star-Forming Sequence at $\mathrm{z}^{\sim} 0.5$ '. ArXiv e-prints, vol.

Saito, T., K. Shimasaku, S. Okamura, M. Ouchi, M. Akiyama, \& M. Yoshida (2006): 'Systematic Survey of Extended Ly $\alpha$ Sources over z 3-5'. ApJ, vol. 648: pp. 54-66.

Santos, M. R., R. S. Ellis, J.-P. Kneib, J. Richard, \& K. Kuijken (2004): 'The Abundance of Low-Luminosity Ly $\alpha$ Emitters at High Redshift'. ApJ, vol. 606: pp. 683-701.

Schaerer, D., M. Hayes, A. Verhamme, \& R. Teyssier (2011): 'Grid of Ly $\alpha$ radiation transfer models for interpreting distant galaxies'. A\&A, vol. 531: A12.

Schaerer, D. \& A. Verhamme (2008): '3D Lyman alpha radiation transfer. II. Fitting the Lyman break galaxy MS 1512-cB58 and implications for Lyman alpha emission in high-z starbursts'. AEAA, vol. 480: pp. 369-377.

Schmidt, W. \& C. Federrath (2011): 'A fluid-dynamical subgrid scale model for highly compressible astrophysical turbulence'. A\&A, vol. 528, A106: A106.

Shibuya, T., N. Kashikawa, K. Ota, M. Iye, M. Ouchi, H. Furusawa, K. Shimasaku, \& T. Hattori (2012): 'The First Systematic Survey for Ly $\alpha$ Emitters at z $=7.3$ with Red-sensitive Subaru/Suprime-Cam'. ApJ, vol. 752, 114: p. 114.

Shimasaku, K., N. Kashikawa, M. Doi, C. Ly, M. A. Malkan, Y. Matsuda, M. Ouchi, T. Hayashino, M. Iye, K. Motohara, T. Murayama, T. Nagao, K. Ohta, S. Okamura, T. Sasaki, Y. Shioya, \& Y. Taniguchi (2006): 'Ly $\alpha$ Emitters at $\mathrm{z}=5.7$ in the Subaru Deep Field'. PASJ, vol. 58: pp. 313-334.

Shimizu, I., N. Yoshida, \& T. Okamoto (2012): 'Lyman alpha emitters in cosmological simulations: Lyman alpha escape fraction and statistical properties'. American Institute of Physics Conference Series. Ed. by Umemura, M. \& K. Omukai. Vol. 1480. American Institute of Physics Conference Series: pp. 412-414.

Shioya, Y., Y. Taniguchi, S. S. Sasaki, T. Nagao, T. Murayama, T. Saito, Y. Ideue, A. Nakajima, K. Matsuoka, J. Trump, N. Z. Scoville, D. B. Sanders, B. Mobasher, H. Aussel, P. Capak, J. Kartaltepe, A. Koekemoer, C. Carilli, R. S. Ellis, B. Garilli, M. Giavalisco, M. G. Kitzbichler, C. Impey, O. LeFevre, E. Schinnerer, \& V. Smolcic (2009): 
'Photometric Properties of Ly $\alpha$ Emitters at $\mathrm{z} \approx 4.86$ in the COSMOS 2 Square Degree Field'. ApJ, vol. 696: pp. 546-561.

Steidel, C. C., K. L. Adelberger, A. E. Shapley, M. Pettini, M. Dickinson, \& M. Giavalisco (2000): 'Ly $\alpha$ Imaging of a Proto-Cluster Region at $\langle z\rangle=3.09$ '. ApJ, vol. 532: pp. 170 182.

Suad, L. A., C. F. Caiafa, E. M. Arnal, \& S. Cichowolski (2014): 'A new catalog of H i supershell candidates in the outer part of the Galaxy'. AESA, vol. 564, A116: A116.

Surlan, B., W.-R. Hamann, J. Kubat, L. M. Oskinova, \& A. Feldmeier (2013): 'Modelling of Resonance Lines in Inhomogeneous Hot Star Winds'. Publications de l'Observatoire Astronomique de Beograd, vol. 92: pp. 197-200.

Tamura, Y., Y. Matsuda, S. Ikarashi, K. S. Scott, B. Hatsukade, H. Umehata, T. Saito, K. Nakanishi, M. S. Yun, H. Ezawa, D. H. Hughes, D. Iono, R. Kawabe, K. Kohno, \& G. W. Wilson (2013): 'Obscured star formation in Ly $\alpha$ blobs at $\mathrm{z}=3.1$ '. MNRAS, vol. 430: pp. 2768-2773.

Taniguchi, Y. et al. (2005): 'The SUBARU Deep Field Project: Lyman $\alpha$ Emitters at a Redshift of 6.6'. PASJ, vol. 57: pp. 165-182.

Tasitsiomi, Argyro (2006): 'Ly-alpha Radiative Transfer in Cosmological Simulations and Application to a z $\sim 8$ Emitter'. ApJ, vol. 645(2): pp. 792-813.

Tilvi, V., J. E. Rhoads, P. Hibon, S. Malhotra, J. Wang, S. Veilleux, R. Swaters, R. Probst, H. Krug, S. L. Finkelstein, \& M. Dickinson (2010): 'The Luminosity Function of Ly $\alpha$ Emitters at Redshift z $=7.7^{\prime}$. ApJ, vol. 721: pp. 1853-1860.

Trac, H. \& R. Cen (2007): 'Radiative Transfer Simulations of Cosmic Reionization. I. Methodology and Initial Results'. ApJ, vol. 671(1): pp. 1-13.

Trowland, H. E., G. F. Lewis, \& J. Bland-Hawthorn (2013): 'The Cosmic History of the Spin of Dark Matter Halos within the Large-scale Structure'. ApJ, vol. 762, 72: p. 72. van Breukelen, C., M. J. Jarvis, \& B. P. Venemans (2005): 'The luminosity function of Ly $\alpha$ emitters at $2.3<\mathrm{z}<4.6$ from integral-field spectroscopy'. MNRAS, vol. 359: pp. 895-905. Vanzella, E., A. Grazian, M. Hayes, L. Pentericci, D. Schaerer, M. Dickinson, S. Cristiani, M. Giavalisco, A. Verhamme, M. Nonino, \& P. Rosati (2010): 'The unusual N IV] -emitter galaxy GDS J033218.92-275302.7: star formation or AGN-driven winds from a massive galaxy at $\mathrm{z}=5.56$ '. A\&A, vol. 513, A20: A20.

Venemans, B. P., H. J. A. Röttgering, G. K. Miley, J. D. Kurk, C. De Breuck, R. A. Overzier, W. J. M. van Breugel, C. L. Carilli, H. Ford, T. Heckman, L. Pentericci, \& P. McCarthy (2005): 'Properties of Ly $\alpha$ emitters around the radio galaxy MRC 0316 257'. A\& A, vol. 431: pp. 793-812. 
Venemans, B. P., H. J. A. Röttgering, R. A. Overzier, G. K. Miley, C. De Breuck, J. D. Kurk, W. van Breugel, C. L. Carilli, H. Ford, T. Heckman, P. McCarthy, \& L. Pentericci (2004): 'Discovery of six $\operatorname{Ly} \alpha$ emitters near a radio galaxy at $\mathrm{z} \sim 5.2$ '. A $\mathscr{E} A$, vol. 424: pp. L17-L20.

Verhamme, A., Y. Dubois, J. Blaizot, T. Garel, R. Bacon, J. Devriendt, B. Guiderdoni, \& A. Slyz (2012): 'Lyman- $\alpha$ emission properties of simulated galaxies: interstellar medium structure and inclination effects'. A\&A, vol. 546, A111: A111.

Verhamme, A., I. Orlitova, D. Schaerer, \& M. Hayes (2014): 'On the use of Lyman-alpha to detect Lyman continuum leaking galaxies'. ArXiv e-prints, vol.

Verhamme, A., D. Schaerer, H. Atek, \& C. Tapken (2008): '3D Lyman Alpha radiation transfer. III. Constraints on gas and stellar properties of $\mathrm{z} \sim 3$ Lyman break galaxies (LBG) and implications for high-z LBGs and Lyman Alpha emitters'. AEGA, vol. 491: pp. 89-111.

Verhamme, A., D. Schaerer, \& A. Maselli (2006): '3D Lyman Alpha radiation transfer'. AछA, vol. 460(2): pp. 397-413.

Wang, Y. (2009): 'Monte Carlo Simulations of Ly $\alpha$ Line Profiles'. Acta Astronomica Sinica, vol. 50: pp. 117-133.

Wyithe, S. \& M. Dijkstra (2011): 'Non-Gravitational Contributions to the Clustering of Ly-alpha Selected Galaxies: Implications for Cosmological Surveys'. MNRAS, vol. 415(4): p. 24.

Yajima, H., Y. Li, Q. Zhu, \& T. Abel (2012): 'ART2: coupling Ly $\alpha$ line and multi-wavelength continuum radiative transfer'. MNRAS, vol. 424(2): pp. 884-901.

Yamada, S. F., S. S. Sasaki, R. Sumiya, K. Umeda, Y. Shioya, M. Ajiki, T. Nagao, T. Murayama, \& Y. Taniguchi (2005): 'An Intermediate-Band Imaging Survey for HighRedshift Lyman Alpha Emitters: The Mahoroba-11'. PASJ, vol. 57: pp. 881-903.

Yamada, T., Y. Nakamura, Y. Matsuda, T. Hayashino, R. Yamauchi, N. Morimoto, K. Kousai, \& M. Umemura (2012): 'Panoramic Survey of Ly $\alpha$ Emitters at z $=3.1$ '. AJ, vol. 143, 79: p. 79 .

Yang, H., J. Wang, Z.-Y. Zheng, S. Malhotra, J. E. Rhoads, \& L. Infante (2014): 'A z 5.7 Ly $\alpha$ Emission Line with an Ultrabroad Red Wing'. ApJ, vol. 784, 35: p. 35.

Zheng, Z., R. Cen, H. Trac, \& J. Miralda-Escude (2011): 'Radiative Transfer Modeling of Lyman Alpha Emitters. II. New Effects in Galaxy Clustering'. ApJ, vol. 726(1): p. 38.

Zheng, Z., R. Cen, H. Trac, \& J. Miralda-Escudé (2010): 'Radiative Transfer Modeling of Ly $\alpha$ Emitters. I. Statistics of Spectra and Luminosity'. ApJ, vol. 716: pp. 574-598.

Zheng, Z. \& J. Miralda-Escude (2002): 'Monte Carlo Simulation of Ly $\alpha$ Scattering and Application to Damped Ly $\alpha$ Systems'. ApJ, vol. 578(1): pp. 33-42. 
Zheng, Z. \& J. Wallace (2014): 'Anisotropic Lyman-alpha Emission'. ApJ, vol. 794, 116: p. 116.

Zheng, Z.-Y., J.-X. Wang, S. Malhotra, J. E. Rhoads, S. L. Finkelstein, \& K. Finkelstein (2014): 'Ly $\alpha$ equivalent width distribution of $\operatorname{Ly} \alpha$ emitting galaxies at redshift $\mathrm{z} \sim 4.5$ '. MNRAS, vol. 439: pp. 1101-1109. 



\section{List of Figures}

2.1 Illustration of a slab of gas with a central source. . . . . . . . . . . . 16

2.2 Lyman- $\alpha$ flux as a function of inclination for a homogeneous slab with an axis ratio of 10 for three cases: optically thick (blue), optically thin (green), optically thick with additional dust content (blue) $\left(\tau_{D} \sim 1\right)$. The scatter in the latter plot comes from the fact that only $6 \%$ of the photons escape the slab in this case. . . . . . . . . . . . . 17

2.3 Correlations of large-scale environmental density (top left), line-of-sight density gradient (top right), line-of-sight velocity (bottom left), and line-of-sight velocity gradient (bottom right) with observed Lyman- $\alpha$ fraction as reported in Zheng et al. 2010. (c) AAS Reprinted with permission (Figure 21 in Zheng et al. 2010) . . . . . . . . . . . . . . . 21

2.4 2PCFs from Zheng et al. 2011 in real space (left column), redshift space (center column), and apparent redshift (right column) for samples of LAEs (first row), halos (second row), and a sample of LAEs for which the Lyman- $\alpha$ properties have been randomly shuffled (last row). The 2PCFs are shown as a function of parallel separation $\left(r_{p}\right)$ and orthogonal separation $(\pi)$. (c) AAS Reprinted with permission (Figure 7 in Zheng et al. 2011) . . . . . . . . . . . . . . . . . . . 24 
2.5 The mechanism causing the observed deformation of the $2 \mathrm{PCF}$ as depicted by Zheng et al. 2011. Dots indicate emitters, the ellipsoids around it the relative flux escaping in a given direction. The background shading illustrates density modes parallel and perpendicular to the observer. Lyman- $\alpha$ photons from galaxies in an overdense region escape predominantly in the direction of the filament, while those from emitters in underdense regions escape along the line of sight. This leads to suppression of modes parallel to the observer and enhancement of modes perpendicular to the observer. (c) AAS Reprinted with permission (Figure 9 in Zheng et al. 2011) . . . . . . . . . . . . . . 25

2.6 Illustration of density, line of sight velocity, and line of sight velocity gradient for a plane-wave density perturbation. . . . . . . . . . . . 26

2.7 Shown are the different contributions to the power spectrum from the radiative transfer effects, parametrized by $C_{\rho}, C_{v}$, and $C_{\Gamma}$. The grey line shows the case where all $C$-parameters are zero. The dashed line corresponds to $C_{\rho} \neq 0$, the solid line to $C_{\rho}, C_{\Gamma} \neq 0$, the long-dashed line corresponds to $C_{\rho}, C_{\Gamma}, C_{v} \neq 0$. Reprinted with permission (Figure 2 in Wyithe \& Dijkstra 2011) . . . . . . . . . . . . . . .

2.8 Shown is the power spectrum as a function of transverse and parallel separation $\left(k_{\perp}, k_{\|}\right)$for the analytical model without any radiative transfer effects (left panel) and for two model including the effects. While the middle panel has $C_{v}<1$, the right panel has $C_{v}>1$, leading to the deformation observed in the simulations by Zheng et al. 2011. Note that the axis here are in $k$-space. Reprinted with permission (Figure 3 in Wyithe \& Dijkstra 2011) . . . . . . . . . . . . . . 29

2.9 Illustration of the Hirata-effect. $\vec{k}$ denotes the orientation of the considered mode of the density field, $\vec{n}$ the line of sight. If galaxies, depicted as ellipticals here, are aligned within the large-scale density field, and the observability depends on the orientation towards the observer, emitter statistics can be systematically affected because the probability of missing a source is higher in certain environments. Reprinted with permission (Figure 1 in Hirata 2009) . . . . . . . . . 30 
3.1 Spectra of a homogeneous, isothermal $\left(T=2 \times 10^{4} \mathrm{~K}\right)$ sphere with an optical depth of $\tau_{0}=10^{5}$ (green), $10^{6}$ (blue), and $10^{7}$ (red). Overplotted in black are the corresponding analytical solutions as obtained by Dijkstra et al. 2006. Taken from Behrens 2011. . . . . . . . . . . . . . 37

3.2 Spectra of a homogeneous, isothermal sphere with Lyman- $\alpha$ photons launched at the center and with a frequency $x=0$. Compared to figure 3.1, a radial velocity field has been added, given by $v_{r}=\frac{r}{R} v_{\max }$. The lines correspond to different choices of $v_{\max }$ as indicated in the legend. Taken from Behrens 2011. . . . . . . . . . . . . . . . 37

3.3 Shown is an illustration of the shell model. The black ring represents a slice through the hydrogen/dust shell moving outwards. Photons are emitted in the center.

3.4 Spectrum of a shell model with parameters $N_{H}=10^{20.2} \mathrm{~cm}^{-2}, T=10^{5}$ $\mathrm{K}, v_{\text {exp }}=300 \mathrm{~km} / \mathrm{s}$, monochromatic point source, no dust. The vertical line marks the typical red peak showing up for this type of model. It results from photons that are scattered at the inner boundary of the shell back into the interior, resulting in a frequency shift that reduces the optical depth. . . . . . . . . . . . . . . . . . . .

3.5 Illustration of a clumpy LAE model. Shown is a slice. The grey area represents the low-density, high-temperature intercloud medium, while the black circles represent dense, dusty clouds. Photons are emitted in the center (yellow star). The Neufeld scenario is illustrated by the two rays. The red one represents the path of a continuum photon. Due to its small cross section with hydrogen, it penetrates the clouds on its way out and therefore is attenuated by dust, and might finally be absorbed. The Lyman- $\alpha$ photon (depicted with a blue line) has a large cross section and therefore rarely enters the clouds. Instead, it bounces off the boundaries of the clouds and leaves the region. As described in the text, this mechanism works only under certain conditions. Note that clouds can have individual velocities, and the emission region can be extended. . . . . . . . . . . . . . . . . . . . . 42 
3.6 Left: flux as a function of angle of observation for the 'density gradient' model for three different values of $A$. The flux is normalized to the isotropic case. Right: coefficients of the multipole expansion of the emerging flux distribution. Reprinted with permission (C) AAS (Figure 2 in Zheng \& Wallace 2014) . . . . . . . . . . . . . . . . . . 44

3.7 Left: flux as a function of angle of observation for the 'velocity gradient' model for three different values of $A$. The flux is normalized to the isotropic case. Right: coefficients of the multipole expansion of the emerging flux distribution. Reprinted with permission (C) AAS (Figure 6 in Zheng \& Wallace 2014) . . . . . . . . . . . . . . . . . . . 45

3.8 Results for the "fiducial" parameter set of Gronke \& Dijkstra 2014. Upper left: projected relative enhancement of Lyman- $\alpha$ escape fraction as a function of the angles $(\phi, \Theta)$. Upper right: projected relative enhancement of continuum escape fraction as a function of the angles $(\phi, \Theta)$. Lower left: projected EW boost $b$ as a function of the two angles. Lower right: cumulative Distribution of $b$ in fractions of sky coverage. The different lines show the result for different sizes of the angular bins, with the darker lines corresponding to smaller bins. The solid vertical line shows the average $\bar{b}$. The red lines show the distribution for the resolution that was used in the other panels,with the dashed lines showing the standard deviation. Reprinted with permission (Figure 1 in Gronke \& Dijkstra 2014) . . . . . . . . . . . . 50

3.9 Results for the "realistic" parameter set of Gronke \& Dijkstra 2014. Left: projected EW boost $b$ as a function of $(\phi, \Theta)$. Right: cumulative Distribution of $b$ in fractions of sky coverage. The different lines show the result for different sizes of the angular bins, with the darker lines corresponding to smaller bins. The solid vertical line shows the average $\bar{b}$. The red lines show the distribution for the resolution that was used in the other panels, with the dashed lines showing the standard deviation. Reprinted with permission (Figure 2 in Gronke \& Dijkstra 2014) . . . . . . . . . . . . . . . . . . 5 50 
3.10 Left: spectrum of the emitter studied by Tasitsiomi 2006, with (dotted) and without (solid line) attenuation by the neutral IGM. The dashed line shows the spectrum with attenuation of IGM only in the blue part of the spectrum, the long-dashed line indicates the rest-frame line center. Right: surface brightness map of the emitter. Reproduced by the permission of the AAS (Figure 10 in Tasitsiomi 2006) . . . . . . . 51

3.11 Surface brightness map for one of the emitters studied by Laursen et al. 2009. Left: excluding dust effects. Right: including dust effects. Reprinted with permission (c) AAS (Figure 4 in Laursen et al. 2009) . 52

3.12 Spectra of the emitters studied in Laursen et al. 2009 for both a dustfree case (dotted) and including dust (solid lines). Reprinted with permission (c) AAS (Figure 10 in Laursen et al. 2009) . . . . . . . . . 53

3.13 The simulated galaxy G2 from Verhamme et al. 2012. Shown is a slice in density (grey contours), the projected number of photon escaping from a region (colored contours) and the velocity field (arrows; scale in the lower left shows amplitude). Reprinted with permission (c) ESO (Figure 7 in Verhamme et al. 2012) . . . . . . . . . . . . . 56

3.14 Escape fraction $f_{\text {esc }}$ as a function of inclination of the escape direction (red) and probability $P$ for a photon to escape if it has an initial inclination $\cos \theta$ (black), shown for continuum (dashed) and Lyman- $\alpha$ photons (solid) as obtained by Verhamme et al. 2012. Reprinted with permission (c) ESO (Figure 8 in Verhamme et al. 2012) . . . . . . . . 57

3.15 EWs as observed along random lines of sight for the simulation in Verhamme et al. 2012, shown as a function of the inclination $\cos \Theta$. The line shows a polynomial fit to the distribution. Reprinted with permission (c) ESO (Figure 10 in Verhamme et al. 2012) . . . . . . . 58 



\section{APPENDIX A}

\section{Contributions to the Publications}

\section{A.1 Effects of Lyman-Alpha Ccattering in the IGM on Clustering Statistics of Lyman-Alpha Emitters}

The code for the simulations was partly written for the Diploma thesis of the author, but completely rewritten and extended in the course of the first months of the $\mathrm{PhD}$, i.e. for the purpose of parallelization and to reduce memory overhead of the used simulation. All simulations, analysis, and figures of the resulting data that were presented in the paper were done by the author during the first year of the $\mathrm{PhD}$, except for Figure 8.1. (a figure showing a primitive code test) which was created for the Diploma thesis.

The text of the paper was initially written by the author, and iteratively edited/revised by Jens Niemeyer and the author. Before submission, the text was revised in minor details after discussing the draft with Mark Dijkstra. The paper was finally revised by the author according to the referee report obtained from the journal.

\section{A.2 Beamed Ly $\alpha$ Emission through Outflow-driven Cavities}

The code for the simulations was completely written on the basis of a stripped-down radiative transfer code, i.e. the Lyman- $\alpha$ radiative transfer physics were integrated by the author in the first/second year of the $\mathrm{PhD}$. All simulations presented were carried out by the author, and the presented models were implemented by the author. The figures were created by the author during his $\mathrm{PhD}$. The draft for the text of the paper was written by the author and iteratively edited by Mark Dijkstra and Jens Niemeyer. The paper was revised according to the referee report obtained from the journal in discussion with Mark Dijkstra and Jens Niemeyer. 


\section{A.3 Inclination Dependence of Lyman- $\alpha$ Properties in a Turbulent Disk Galaxy}

The radiative transfer simulations were performed using the code mentioned in A.2. All the simulations, analysis, and plotting was carried out by the author. The text was written by the author and edited/supplemented by Harald Braun. Before submission, the text was revised according to discussions with Jens Niemeyer. The paper was edited by the author in discussion with Harald Braun and Jens Niemeyer according to the referee report obtained from the journal. 


\section{APPENDIX B}

\section{A Non-exhaustive List of LAE Detections}

Table B.1: Incomplete list of LAE detections at redshifts $>2$. We note that the notion of a "confirmed" LAE depends on the used observation technique and the criteria employed to remove interlopers. We quote the number of detections according to the publications. We cannot rule out that some objects were reported in more than one publication without notice. Therefore, we make a conservative estimate here for the total number of detections. Formally, the sum of detections (including possible double-detections) is 5868. The list was partially compiled using the list in Barnes et al. 2014.

\begin{tabular}{lll}
\hline Author & \# of objects confirmed & Redshift \\
\hline (Hu \& McMahon 1996) & 2 & 4.4 \\
(Pascarelle et al. 1996) & 2 & 2.4 \\
(Hu et al. 1998) & 19 & $3.4-4.5$ \\
(Kudritzki et al. 2000) & 9 & 3.1 \\
(Steidel et al. 2000) & 72 & 3.09 \\
(Rhoads \& Malhotra 2001) & 18 & 5.7 \\
(Hu et al. 2002) & 1 & 6.6 \\
(Ajiki et al. 2003) & 2 & 5.7 \\
(Kodaira et al. 2003) & 2 & 6.5 \\
(Ouchi et al. 2003) & 87 & 4.9 \\
(Rhoads et al. 2003) & 3 & 5.7 \\
\hline
\end{tabular}

continued on next page... 


\begin{tabular}{|c|c|c|}
\hline Author & \# of objects confirmed & Redshift \\
\hline (Dawson et al. 2004) & 17 & 4.5 \\
\hline (Hayashino et al. 2004) & 283 & 3.1 \\
\hline (Hu et al. 2004) & 19 & 5.7 \\
\hline (Kurk et al. 2004) & 1 & 6.5 \\
\hline (Rhoads et al. 2004) & 1 & 6.5 \\
\hline (Santos et al. 2004) & 11 & $2.2-5.6$ \\
\hline (Venemans et al. 2004) & 6 & 5.2 \\
\hline (Taniguchi et al. 2005) & 7 & $6.5-6.6$ \\
\hline (van Breukelen et al. 2005) & 14 & $2.3-4.6$ \\
\hline (Venemans et al. 2005) & 31 & 3.3 \\
\hline (Yamada et al. 2005) & 198 & $3-5$ \\
\hline (Gawiser et al. 2006) & 18 & 3.1 \\
\hline (Kashikawa et al. 2006) & 8 & 6.5 \\
\hline (Saito et al. 2006) & 41 & $3.2-5.0$ \\
\hline (Shimasaku et al. 2006) & 28 & 5.7 \\
\hline (Dawson et al. 2007) & 59 & 4.5 \\
\hline (Gronwall et al. 2007) & 162 & 2.1 \\
\hline (Nilsson et al. 2007) & 3 & 3.15 \\
\hline (Finkelstein et al. 2008) & 4 & 4.4 \\
\hline (Ouchi et al. 2008) & 84 & $3.1-5.7$ \\
\hline (Rauch et al. 2008) & 27 & $2.67-3.75$ \\
\hline (Grove et al. 2009) & 83 & $2.8-3.2$ \\
\hline (Nilsson et al. 2009) & 170 & 2.25 \\
\hline (Shioya et al. 2009) & 79 & 4.7 \\
\hline (Guaita et al. 2010) & 250 & 2.1 \\
\hline
\end{tabular}




\begin{tabular}{lll}
\hline Author & \# of objects confirmed & Redshift \\
\hline (Hayes et al. 2010) & 38 & $2.2-5.6$ \\
(Hibon et al. 2010) & 7 & 7.7 \\
(Hu et al. 2010) & 118 & $5.7-6.5$ \\
(Ouchi et al. 2010) & 207 & 6.6 \\
(Tilvi et al. 2010) & 4 & 7.7 \\
(Adams et al. 2011) & 105 & $1.9-3.9$ \\
(Cassata et al. 2011) & 217 & $2.2-6.62$ \\
(Dressler et al. 2011) & 122 & 5.8 \\
(Barger et al. 2012) & 28 & $0.67-1.16$ \\
(Ciardullo et al. 2012) & 104 & 3.1 \\
(Hibon et al. 2012) & 8 & 7.3 \\
(Nakajima et al. 2012) & 919 & 2.2 \\
(Krug et al. 2012) & 4 & 7.7 \\
(Shibuya et al. 2012) & 4 & 7.7 \\
(Yamada et al. 2012) & 2161 & \\
\hline Yang et al. 2014) & 1 & 5.7 \\
\hline
\end{tabular}





\section{Publications}

\section{Scientific publications}

1. Behrens, C. \& J. C. Niemeyer (2013): 'Effects of Lyman-alpha scattering in the IGM on clustering statistics of Lyman-alpha emitters'. ApJ, vol. 556: A5.

2. Behrens, C. \& H. Braun (2014). A\&A, vol. accepted.

3. Behrens, C., M. Dijkstra, \& J. C. Niemeyer (2014): 'Beamed Ly $\alpha$ emission through outflow-driven cavities'. A\&A, vol. 563: A77. 



\section{Curriculum Vitae}

\section{Personalien}

Name

Christoph Behrens

geboren am 05.03.1985

verheiratet, deutsch

\section{Schulbildung}

2004

Abitur, Gymnasium Julianum in Helmstedt

\section{Studium}

2005 - 2011 Universität Göttingen, Studium der Physik

Thema der Diplomarbeit: 'Numerische Simulation des Transports von Lyman- $\alpha$-Strahlung auf kosmologischen Skalen', durchgeführt am Institut für Astrophysik

2006-2012 Universität Göttingen, Studium der Philosophie

Oktober 2011 Abschluss: Diplom-Physiker

Januar 2012 Abschluss: 2-Fach-Bachelor Physik/Philosophie studium generale

\section{Promotion}

Oktober 2011 - heute Wissenschaftlicher Mitarbeiter am Institut für Astrophysik, Universität Göttingen 



\section{Acknowledgments}

I would like to thank Jens Niemeyer for giving me the opportunity to write my $\mathrm{PhD}$ thesis, for his advisory, and in particular for his comments on the drafts. I would further like to thank Wolfram Kollatschny and Dominik Schleicher for their support. Also, I have to thank Mark Dijkstra for numerous useful discussions. A special thanks goes to Jeff Engels and Harald Braun, who where my office mates during the last four years and always provided help and - at rare occasions - even the small piece of distraction people sometimes need, and of course to all the other former and current members of the cosmology group.

I would like to thank my wife Jelka for countless little things that cannot be mentioned here, but in especially for - I have to copy this line from my Diploma thesis - not letting me go nuts, but also for many helpful discussions and comments during the last three years.

This thesis was written using a Latex template compiled by Matthias Pospiech.

Post scriptum: To counteract the rather histrionic epitaph in the beginning of my thesis, I would like to conclude with a different quote:

(Sheldon Cooper, a brilliant theoretical physicist, refusing to go to a fundraising party of the dean)

Sheldon: Tell him Dr. Cooper feels that the best use of his time is to employ his rare and precious mental faculties to tear the mask off nature and stare at the face of God.

Penny: Sheldon, it's Saturday night, you'll be doing laundry.

from the series 'Big Bang Theory' 
